\title{
Lapurdum
}

Euskal ikerketen aldizkaria | Revue d'études basques |

Revista de estudios vascos | Basque studies review

Numéro spécial 4 | 2019

SACAZE bildumako euskal testuak (1887)

\section{Bildumako euskal testuak}

\section{Gotzon Aurrekoetxea, Charles Videgain et Aitor Iglesias Chaves}

\section{(2) OpenEdition \\ Journals}

Édition électronique

URL : https://journals.openedition.org/lapurdum/2897

DOI : 10.4000/lapurdum.2897

ISSN : 1965-0655

Éditeur

IKER

Édition imprimée

Date de publication : 1 octobre 2019

Pagination : 37-205

ISBN : 9782955341339

ISSN : $1273-3830$

\section{Référence électronique}

Gotzon Aurrekoetxea, Charles Videgain eta Aitor Iglesias Chaves, «Bildumako euskal testuak», Lapurdum [Linean], Numéro spécial 4 | 2019, Sarean emana----an 12 janvier 2020, kontsultatu 24 septembre 2022. URL: http://journals.openedition.org/lapurdum/2897 ; DOI: https://doi.org/10.4000/ lapurdum.2897

\section{(c) (i) (9)}

Creative Commons - Attribution-NonCommercial-NoDerivatives 4.0 International - CC BY-NC-ND 4.0 https://creativecommons.org/licenses/by-nc-nd/4.0/ 


\section{BILDUMAKO EUSKAL TESTUAK}

Testuen hurrenkera Sacazek emandakoa da. Hala ere, eta modu errazago batean ulertzeko testu guztiei antolamendu bera eman zaie:

a) Testu-burua edo orrialde-burua agertuko da lehenik, koadro baten barnean. Bertan ondoko informazioa ematen da :

- Herriaren izendapena frantses eta euskal grafian emana

- Kantonamendua, parentesi barnean emanik

- Populazio kopurua: testua itzuli zen garaian herrian bizi zen jende-kopurua

b) Testuak elkarren ostean

c) Testuari dagozkion oharrak (herri zenbaitetan)

d) Testuaren itzultzailea eta hari dagozkion zehaztasunak 
ARCANGUES / ARRANGOITZE (Canton de Bayonne) Population: 1040

Baciren lehenago Pyreneko herri batian guizon eta emazte bat arras zaharrak. Etzuten etche ttipi bat, baratce bat eta behi bat baïcik. Baïnan, nahis beharrak ciren, laguntcen cituzten bethi hec baïno beharrago cirenak. Egun batez, lurra elhurres estalia cen, eta horma ari harriac arrailtceko. Herriko yende guciak berotcen ciren; yaten eta edaten hari cirelarik; aberatx eta urus ciren. Ilhun heguian, bi piyayant urrundic heldu cirenac, nahi izan zuten guelditu lekhu hartan, hotz eta gose cirelakotz. Yo zuten athe bat, guero bertce bat, eta oraino bertze batzueri: nihoc etcitin sartzerat utci nahi izan, ez deüsik yaterat eman. Kasatuyak orotaric, chakhur gaisto batzu ondotik, bi bidekariek etxaquiten norat yoan, heldu cirenean herriaren bururat, beharrak ziren guizon eta emaste heyen etchiaren ondorat. Hekien errenkurak aintxiarequin, senharra eman tsen leihoan hekien deitceko, eta emastiac laster eguin zuen athiaren idekitcerat. Galdatu gabe nor ciren, sararazi zituzten eta yarrarazi su pasterrian; eskaïni ciozkaten yaterat esnia eta cembait gastaïna, zuten guzia. Bainan orduyan Salbatzaïlea - hura zen Yondoni Petrirekin - buruya distirant, eta érran zioten: "Zuik beharrak eta onac zarete; zuin haüsoac aberatxac eta gaichtoak, zuzen dena eguin bedi!”. Haïn bertcenarekin, ikharatu cen lurra Salbatxailea eta Yondoni Petri ezeztatu ciren eta bi zaharrak belhaüniko erori. Biharamun goïzean, yoan nahi izan zirenean herrirat, cer iragan cen yakiteko, etzuten ikusi etche bihirik, hoyen lekhuyan, estaïnu bat cen, egungo egunian han berian kaüsitcen dena Barbazango herriaren eta Saint Bertrand de Comminges deitcen den hiriaren artian.

Behar dire sokhorritu eskasian direnak.

\section{oOo}

Tantugou da zahar bat oihanian gordetcen dena, zaïntcen ditu landac eta sorhoac deüs ez dezaten ebatx. Oguiak eta belharrac pikatuyac direnian; Nausiak oro barnerat eman artio Tantugou ez da lokhartcen; bethi atxarria dago. Hura aguertcen ikhus beldurrak daüska ohoïnak. Ona da, sinhetx zazue, baïna basa da. Asko gauza zakien laborari batek bere semieri erraiten cioten maïz Tantugou cela segurki lurreko bihien zaintzaile hoberena.

Ustaileko arratxalde batez arthalde bat alha cen mendi baten gainean; artxaina lokhartu cen: iratxartxiarekin, ikhusten du Tantugou oïhanerat ihesi zohala. Izan txen oro bat ezagutua artxainez, haran eder batian, etxana harroka baten gaïnian.

Egungo egunian oraïno Pyreneko herri cembeitetan, haurrac haren beldur dire. Muthiko edo nechka ttipi batec bere ama obeditu nahi ez duenian, erraïten dio: Kasu eman! oỉhu eguiten diat Tantugouri, eta couchian, yinen duk oïhanetik!

$\mathrm{B}^{\mathrm{din}}$ Abeberry, 40 ans, Arcangues 
Lehenago baziren senhar emaste batzu, mendietaco herri batian. Ez zuten etche ttipi bat baatziakin eta behi bat baizic. Bainan, zembat nahi pobre izan ziten, lagutzen zitusten berse berac baino pobriagoak. Egun batez, lurra estalia zen elhurrez, horma beltza edo carrogna ari zuen: Herrico yende guziac berotzen tziren, yanez eta edanez. Irusac eta abaatsak zien. Ilhuntsian, bi piayant, urrundic heldu zienac, nahi izan zuten guelditu toki [?] herri hartan, zeren baitzien gose eta hotz. Yo zuten bortha batian, geuro bertzeatian eta guero bertzietan eraino, nehoc ez ziusten nahi utzi sartzeat ez deusik eman yateat. Casatuyac guzietaïc, pertseytuïac sakkur oldartuyez, bi arrotz hec, ez zakiten noat yoan; noiz eta khausitu baitzien herrian zolan, guizon eta emazteki pobre heyen bothaan aitzinian. Heyen plainuyac aitciaekin, senhara leyhoat atheatu zen, heyer oïhu eyteko, emaztia yuaiten zelaïc laster borthain idekitzeat. Galdatu gabe nor zien, sarraazi eta yarrarazi ziuzten bere supasterrian, guero zerbitzatu ziozcaten esnia, eta zenbait gazteïnac; ziuzten gusiak bainan orduyan Jesu Cristo gure Jaunac - hura zen Jondoni Pétriekin - altchatu zen, buruya distirant eta erran zioten: Zuic pobriac zaïzte eta onac, zuin aüsuac dire abaatsak eta gaichtuac "Jinkuan yustizia ein dadila". Berreala lurra ikharatu zen Jesus eta Jondoni Petri suntsitu zien eta bi chaharrac erori zien belhaunico.... Bihamun goizian, nahi izan zutenian yuan plazaat, yakiteco zer phasatu zen, ez zuten ikhusi etche bihiic: etchien plazan zen phutzu handi bat zoina causitzen baitza oïno Barbazan deitzen den herrian eta St Bertrand de Comminges hiriaren artian.

Behar dire maluusak soleitu.

oOo

Tantugou da guizon zahar bat oïhanetan gordetzen dena. Gardiatzen tu landac eta phentziac amore gatic deus ez daïn han ebats. Oguiac picatuac eta phentziac eakiak dienian, errecolta guzian nausiac sart artio, Tantugou ez loharcatzen, bethi iratzarria da. Haïn ikhusteko beldurrac ohoinak urruntzen tu. Ona sinhets zazii, bainan salbaya. Laborari, hanitz gauza zakiin batec, erraiten zioten maiz bere haurrei Tantugou zela segurki lurreco frutuen zeïntzaileik hobeena.

Uztaileco atsalde batez, arthalde bat alhatzen zen mendi batian, artzaina lokhartu zen, eta iatzartziakin, ikhusi ziin Tantugou ihes zohala oïhaneat buruz. Tantugou ezaütua izan zen berdin celhay pollit batetaco artzainez, etzana harroca baten gainian.

Oaïno oaïco egunian, Borthuetaco zembait herrietan haurrac haren beldur die. Muthico edo nechcato tipi batec ez diinian obeitu bere amari, hunec erraiten dio: Gardia emac! Ekharrazico diat Tantugou, eta berheala, yinen duc oihanetic.

Duclos, né le 9 août 1849 à Hélette Coll.: Garat Jean, né en 1804 à Bassussarry, y domicilié de tout temps 
Bacen léhénago Pyrénéétaco herri batian guiçon bat éta émaztékibat biciki saharrac. Ez çouten étché ttipi bat, baratcé bat éta béhi bat baïcic. Bainnan cembat nahi pobré içan siten, béthi lagountzen citouzten hec baïno pobriagoac. Egoun batez, lourra estalia sen elhourrez, éta horma ari cen harriac ourrastéko artian. Hérrico yendé gouciac bérotzen hari ciren. Ilhoun héguian, bi piaiant, ourroundic heldu ciénac nahi izan ciren guelditou toki hartan, céren gossé éta égarri baïtziren; yo çouten bortha batéan, guéro bertzé batéan éta oraino bertzé batzuétan, néhorc ez citouzten outzi nahi içan sartzéra, ez éta héierri déousic éman yatéra. Cassatouac orotaric, sakhourrez séguitouac, coléra handitan, bi estranyer hoyec ez sakiten norat yoan, noïz éta ére khaoussitou baïtziren herriaren bourouan, pobré bici ciren guiçon éta émazteki hoyen etchéaren aitzinéan,. Héyen plaïnouac aditziarékin, guiçona yoan zen leyhorat héien deitzéco, eta haren émaztia yoan cen fite bortharen idékitzéra. Héierri galdéguin gabé nor ciren, sar araci ciuzten eta eta yar arazi sou pasterrian; guéro cerbitzatzou cioten esnia éta gazténa combeit hec çuten goucia. Baïnan ordouian, Yaincoua, - céren houra zen Yondoni Petirirekin - choutitou cen: bourouia déna dichtiratzen ciona, éta erran cioten: "çouec pobré eta on çaresté; çouen aouçoac aberaxac éta gachtoac diré. Yincoaren youstisia éguina izan dadila!". Hainbertzénarékin lourra ikharatou cen, Yésous éta Yondoni Pétiri sounsitou ciren, eta bi çaharrac érori ciren belhaunico... Biharamoun goïcian, noïz éta éré yoan nahi içan baitziren herrirat, yakitéco cer passatou cen, ez çouten ikhoussi etché bihiric: héien plaçan, bacen our bildou bat, oraïno khaussitzen dena égoungo égounian, Barbazanéco herriaren eta Saint-Bertrand-de-de-Comminges icéna douen hiriaren artian. Lagoundou béhar diré pobriac.

\section{oOo}

Tantugou, guiçon çahar bat bat da çoïna gordétzen baïta oihanétan. Saïntzen tou landac eta pentziac han déoussic ébax ez déçaten. Ogui éta bélhar gouciac pikhatouac dirénian, dénac naoussiac barnérat éman artino, Tantugouc ez dou loric éguiten, gouardian da béthi. Haren aguertzen ikhoustéco beldourrac atchikitzen ditou ohoïnac. Ona da, sinhex çaçoue, baïnan salbaïa da. Laborari batec, haïnitz gaouça çakinac erraïten çouen maïz béré sémerri Tantugou céla ségourki lourréco frouitouen caïntzaléric hobéréna.

Ouztailé hilabétéco arratxalde batez, ardi tropa bat alhan ari cen mendi batéan; artzaïna lokhartou cen. Lotaric iratçartcéan ohartou cen Tantugou ihessi yoaiten céla oïhaneco aldérat. Eçagoutouya içan cen orobat artzaïn batzouez planou pouillit batian, etzana arroca baten gaïnian.

Oraino égoun Pyrénéétaco herri batzouétan haourrac haren beldour dira. Mouthico ttipi batec edo nescatoco ttipi batec sinhetsi nahi ez douénian béré amari, hounec erraïten dio: Gouardia hiri! Oihou éguiten diat Tantugouri, eta, ichtantian, yinen douc oỉhanétic!

Bordato, 49 ans, né à Barcus. Coll.: Detcheverry Henry 
Baziren lehenôo, Pyréneac deitcen diren mendi haütaco herri batetan, guiçon eta emastekibat bicik caharrac c-irenac. Ez çuten etché ttipittobat, baratcebat eta behi bat baïcic. Baïnan haïn beharrac içanâtic, sokhorritcen ciuzten bethi berac baïno beharretanâo cirénac. Egun batez, lurra elhurrez estaliacen eta haïcen horma arraillki. Herrico yende guciac supasterrean berotcen çaüdelaric, haïciren yaten eta édaten, aberats eta irus ciren. Ilhoun héguian, bi piyayant urrundic heldu cirenac, nahi içan ciren guelditu toki hartan, ceren eta hotz eta gose baïciren. Yo zuten bortha bat, guero bertcebat, eta bertcebatçûtan ôano; néhorc ezciuzten nahi içan barnerat sartcera utci, ez eta éré deusic jaterat eman. Orotaric casatuyac, çakhur çoratubatçuez ondotik séguituyac, bi arrotzec ez çakiten norat joan noïz eta ere caüsitu izan baïciren herriaren baztéreean, guiçon eta émastéki beharretan ciren heiyen etchearen aïtcinean. Aitciarekin heien hatsgorapenac??? senharra eman cen leihoan heyerri oïhy éguiteco eta laster emaztea joan cen bortharen idekitcera. Nor ciren galdeguin gabé, sarraraci cituzten eta yarraraci beren supasterrean; guero cerbitçatu cieten esnea; çombait gaztena, cituzten guciac. Baïnan orduyan Jinco, gure Jaüna, beracen Yondoni Pétirikin; chutitu cen buruya çuelaric distirantasun handibatetan, eta erran içan çakoten: "Ciek béharrac, eta onac ciezte, cien aüçoak aberaxac et gaïstoac dire, Jaïncoaren yusticia eguin bedi!”. Orduyan béréan lurra ikharatucen, Jesus eta Yondoni Petiri bistatic galdu içan ciren, eta bi çaharrac érori ciren belhaüco... Biharamun goïcéan, nahi içan cirenean herrirat yoan jakiteco cer phasatu içan cen, ezcuten etcheric batere ikusi: heyen lekhuyan caüsitu ican çuten ur gueldi hil bat, egungo egunean ikhusten dena, Barbazaneco herriaren eta yondoni Petiri-dé-Commingesco hiriaren artean. Beharrac behar dire lagundu

\section{Observations.}

Il n'y a pas de combinaison de voyelles en basque.

Prononcez le j et l'y comme dans les mots royaume, royauté.

Prononcez l's grassement comme dans les mots espagnols segnor, seguro.

Prononcez le z comme le s français.

La voyelle $u$ a la le son de ou.

Le double tt se prononce à peine, doucereusement, comme quand vous appelez les poussins, tti... tti... tti...

Il n y a pas d'e muet. Prononcez l'x comme tsa.

oOo

Tantugou da çaharbat oïhanetan gordetcen dena. Ez deçaten deus ebats, çaïntzentu landac eta pentceac. Oguiac picatuyac direnean edo belharra ebakia, naüsiak oro etcheratu 
artio, Tantugouk ezdu seculan loric; çaindago bethi. Haren ikhusteco beldurkundeac atchikitcentu ohoïnac. Prestuya da sinhex çaçu baïnan basa da errabiatuya. Laboraribatec; asco gaücez yakinsun cenac erten çakoten maïz bere semerri; Tantugou cela segur lurreco fruituyen çaintçaleric hoberena.

Uztail hilhaïteko eguerdi ondo batez, ardi arthalde bat alha cen mendibatetan; artçaïna lokhartu cen: iharçatu cenean ikhusi cin Tantugou oïhanaï buruz ihesi yohan cela. Bardin éçagutuya içan cen arçaïnez erpira ttipy pullit batétan, etçana arrocâten gaïnean.

Egungo egunean oano, Pyrénéac deitcen diren mendietaco coïmbaït herrietan, haurrac haren beldur diré. Noïz eta ere, muttico ttipi edo nescato ttipi batec, ezbaïtu amari obeditu nahi ertendaco: Eissac khasu (pour un garçon), Eisan khasu (pour une fille), oihu éguinen diat (dinat pour une fille) Tantugouri eta berehala yinen duc (ou doun) oïhanetic.

(Mêmes observations qu'en l'autre part)

Celhay, 54 ans, né aux Aldudes. Coll: Mendiboure Victor, Bassenave Bernard. 
Baciren lehenago Pyrene mendietaco herri batean guiçon bat eta emaztequi bat, arras çaharrac. Ez çuten etche ttipi bat baratcearequin eta behi bat baicic. Bainan nahi becen pobre içanagatic laguntcen cituzten bethi berac baino pobreagoac. Egun batez lurra elhurrez estalia cen, eta haicen harriac berac erdiratu nahi cituen hotz bat. Herrico yende guciac berotcen çauden yaten eta edaten. haricirelaric. Aberats ciren eta dohaxu. Ilhuntzebatez bi arrotz urrundic heldu cirenac, nahi içan çuten lekhu hartan guelditu, hotçac eta goseak yoac cirelacoan. Yoiten dute behin athe bat, guero bertce bat, azquenecotz hainitz athe. Nihorc ez cioten atheric idequi ez eta deus yaterat eman nahi içan. Orotaric egorriac, çakhur gaichto batçu ondotic, bi arrotz horiec ez çaquiten norat itçul, noiz-eta-ere herretic atheratcean causitubaitciren guiçon eta emastequi pobre hoiquien etchearen aintcinean. Hoiquien oyhu minac aitcearekin Saharra leihoan emantcen heguien ekharrazteco, eta berehala emaztea yoan citçayoten athearen idequitcerat, nor ciren galdetu gabe sarraraci cituzten eta yarraraci bere sukhaldean eta emancioten esne, gaiztaina eta cituzten gauça gucietaric. Bainan orduan gure yauna Gure-Jauna- hura bera cen Jondoni Petrirequin- chutitcen da buru distirant batequin eta erraiten diote: çuec pobre çarete bainan bihotz ona duçue: çuen hauçoac aberatzak dire bainan gaichtoac. Jaincoaren justicia eguin dadiela. Orduan berean lurra ikharatcen da, eta GureJauna eta Jondoni Petri hequien beguietaric itçaldu ciren; eta bi çaharrac ahuspez belhaunico eman ciren. Biharamun goicean yoan nahi içan çutenean herrirat yaguiteco cer guerthatucen, ez çuten etcheric batere icusi; bainan hequien toquian causituçuten ur handibat. Oraino gure demboretan agueri dena Barbazaneco herriaren eta San Bertrand de Commingeco hiriaren artean. Dohacabeac behar dire lagundu.

\section{oOo}

Tantugou guiçon çaharbat da, oyhanetan gordet-cen dena. Landac eta sorhoac çaintcen ditu, ez deçan nihorc deus ebats. Oguiac eta belharrac nausiec picatcen dituz tenean barnerat oro sarthu arteraino Tantuguc eztu loric eguiten: bethi atçarria eta erne dago. Beldurrez hark ikus, ohoinak barna daude. Ona da; sinhesten ahal duçu; bainan oyhanetan bici direnac beçalacoa da hainitz gauça baçaquien. Laborari batec, erraiten cioten maiz bere semeri Tantugu cela segurqui lurreco fruituen beiratceleric hoberena.

Uztaileko arratsaldebatez arthaldebat alha cen mendibatean. Artçaina lokhartucen, atçartzean icusi çuen Tantugu ihesi çohala oyhanerat.

Bertce artçain batçuec bardin eçagutu çuten haran pollit batean etçana arroca baten gainean. Oraino egungo egunean Pyrenetaco herri bakharbatçuetan haurrac haren beldur dire. Muthico ttipi batec edo nescatcha ttipi batec ez duenean bere ama obeditu nahi, erraiten dio: Beirauc oyhu eguiten cioat Tanguguri; eta berehala heldu duc oyhanetic.

Armand Sourrouille, 20 ans, né à Bergouey Collaborateurs: néant 
Bazen leheno, Pyrenees deitzen duten menditan gizon bat eta emaztet arras zaharac. Etz zuten etche ttipi bat bezin, baatzet eta behi bat. Bana nahi bezen probe izan zitain, berac beno probio zienak sokhoritcen ziuzten. Eun batez, lurra estalii zen elhurrez eta horma haizen harri urarto. Herriko yende guzik berotzen ziutzun, yatian eta edatian, abaatsak ziutzun eta irusak. lhuntzian bi boyajur urunetik heldu zielaik, nahi zien gelditu lekhu hartan, zeren eta hotz eta gose beitzien; bortaatetan yo zuten, eta bezeatetan eta bezetan oraino: nehok eziuzte utzi nahi ukhan sartze, eta ez eman nahi ukhan deusik yate. Ootaik khasatuik, bee ondotik ukhanez zakhur samurtuik, bi estranyerek etzakiten noat yoan, noiz gertatzen beitzien herrian buruin gizon eta emazte heyen etchean borthan. Heyen plainuik aitzian, gizona emantzen leihun hoyerri oihu eiteko, eta haren emaztia yuanzen fite borthan ideite. Galdeïn gabe nor zien, sarazi zuzten eta yareazi su phazterin, gero zerbitzatu zaizten esnia eta gaztenak, zuzten guzik. Bana orduin, Gure Yuhkua - hua zuzun Yundane Petrikin- yeyki zen buruik argitzen zakolaik, eta eran zeiezun: "ziek probik zizte, eta onak; zien auzuk, abaatsak tutzu eta gaichtuk. Yunkuan yuztizia eindaila!”. Ordu beh lurra ikhaatu zen, Yesus eta Uundane Petri galdu zien, eta bi zaharc eroi zien belhaunku... Bihamen goizin, yoan nahizan zienin plazalat yakiteco zer phasatu zen, etche bihik etzizien ikhusi, hein lekuin bazen hur handi bat, oano hatzamaiten dutena eun Barbazaneco herrian eta San-Betran-de-Comminyeko hirian artin. Behar da maleusei sokhorri eman.

\section{oOo}

Tantügou duzu oihanetan gordatzen den gizon zaharbat. Zaintzen titzu landak eta sorhuk deusik ebatsak eztezaten. Ogi phicaturik denin edo belharra phicatuik, dena nausiz bilduik izan arte Tantugou eztuzu gehiago lo; bethi beilatzen dizu. Haren ikhusteko beldurrak atchikitzen tu ohoinak. Ona uzu sinhetsazu, bena basa uzu. Hanitz gauza zakin laboraribatek erten zizun usu bee semeri, Tantugou zela seurki lurran gaineko fruituien gardienik hoberena.

Uztailako aatsaldeetez, ardi tropat mendi baten gainin phasatzen zuzun, artzainak lok hartu zuzun; idatzartzian, aperzeitu zuzun Tantügou oihaneat buz. Ibar ${ }^{1}$ eder bat etan, izan zuzun bardin ezauturik arrokaaten gainin.

Oano eun, Pyreneeko zombeit herritan, haurrak haren beldur tutzu. Muthiko edo nexkatoko ttipi batek eztinin bee ama sinhetsi nahi, erten dakozu: "Eizak² khasu! Oihu eiten diat Tantügoui ta orai bein yen duk oihanetik".

${ }^{1}$ Ibar, Ibarra: nom souletin de la vallée. Ce terme n'est pas connu dans la commune. Aussi pour compléter la phrase ai-je cru devoir employer le terme souletin

2 S'il s'agissait d'une petite fille, on dirait: Eizan khasu! Oihu eiten dinat Tantügoui eta orai bein yen dun oihanetik. 


\section{Explications sommaires}

Le Pays basque se divise en trois parties: le Labourd, la Basse-Navarre et la Soule. Chacune de ces parties a son dialecte ou son idiome propre. Ainsi à part quelques mots qui ne sont pas les mêmes dans les deux autres langues, la langue souletine se distingue par la douceur des sons. Les Labourdins n'ont pas de $u$. Ils prononcent cette voyelle comme les Espagnols ou. Les Souletins l'appellent aussi parfois ou comme dans hua eau mais ils lui donnent aussi dans certains cas le son de l'u comme dans ürzua (palombe). Mais l'u est surmonté d'un tréma.

Urcuit (Urkheta) étant dans le Labourd, tous les $u$ du texte se prononcent ou. Cependant pour garder en basque au mot Tantugou la prononciation du français, ce mot n'étant pas basque d'ailleurs, j'ai adopté l'orthographe du basque souletin.

Le $c$ s'écrit $k$ en basque.

Le $g$ n'a jamais le son de $j$, même devant les voyelles.

On ne se sert jamais du $\mathrm{j}$ dans le basque labourdin comme cela se fait dans le basque souletin. Le $j$ se remplace ici par $y$.

Le s n'a pas en basque la prononciation du français. Pour le prononcer, il faut faire entendre un petit sifflement.

Le son de $s$ français est représenté en basque par la lettre $z$.

Le $p$ placé avant $h$ se prononce séparément de cette dernière lettre.

Quand dans un mot se présente le double tt, on mouille ces deux lettres.

Le $i$ après une voyelle se prononce toujours séparément de cette dernière et ne prend jamais de tréma. 


\section{Barbazaneco guerthakaria}

Baçuçun lehenago Pyrénéétako herri batetan guiçoun bat eta emazteat anhitz çaharrak béitcien. Etsicien etche ttipi bat, batceat eta behi bat beicik. Bana çoumbat nahi praoué issaniké, bethi sokhorritcen sitcien berac bano praouyagouac cienak. Egoun batez, lurra estallia çuçun elhurrez, eta kharrougna ari harriac erdiratu arte. Herrico yendiac oro beotcen çutçun yatiakin eta edatiakin; abatz eta iruz çutçun. Gaoua sarsiakin, urhenetik heldu cien bi piaiant, nahi hiçan çutçun lekhu hartan guelditu, cen eta gosse eta egarri beitcien; yo cicien borthabatetan, gueo beceatetan, eta ono beze anhitsetan: etcicien nehorkek sarreci nahi, ez eta yateat eman nahi. Ootaïk khassatatiak, sakhur samur batsuz seguitiak, bi estranyerek eshakicien nouat youan nouiz eta khaussitu beitcien herrian burien, guiçoun eta emazte praouiak cien heien etchian aitcinien. Ensutiakin heien plagniak, senharra eman çuçun leihouen heier oihu eiteko, eta han emaztia youan çuçun fite borthan idoïteat. Galdatu gabe heier çougn cien, sarreci citcien eta yarreci heien supasterrien; guero serbitsatu çakocien ezne eta gaztagna çoubeit, heiek cuztenak oro. Bena ordien, goue Yainco Yaouna, houa beitcen saint Pierrekin, yeki çuçun, buria dena dirdiretan, eta erran çakocien: "Siek, praouyac eta ounac ciezte; cien aïssokouak abatsak eta gueistouak. Yaincouaren yusticia ein daïla!”. Behala, lurra ikhatu çuçun, Yéssus eta saint Pierré itsaldu eta bi çaharrak belhoinko erori. Bihamoun goïcien, plaçat youan nahiçan cienien, yakiteko ce phassatu cen, etcicien etcheik bihibat ikhoussi heien plaçan, baçuçun ossin bat, egoungo egounien khaoussitcen dena Barbazaneko herrian eta Dona Bettan Commingueko hirian artien. Behaïci laoundou maleroussak.

\section{Tantugoun guerthakaria}

Tantugou uçu oyhenetan gordetcen den atsoat. Beyratcen titsi elguiak eta phinsiak amokatik eta ez deçaten yeousik ebatz. Oguiak phikatiak dienien edo belharra ebakia, Tantugouk etci bate loïk eïten naoussiak denak erretiatu arte; bethi beillatcen dici. Han aguersian beldurrak ouhougnak achiten titci. Ouna üssu, sinhetz çacie, bana salbaya. Anhitz gaouça çakien laourari batek, bere semer ussu erreiten cicin, Tantugou cela seurki lurreko frutien beiraçale hobena.

Ustailla hillete egouerdi ondo atez, ardi tropat mendi baten puntan alhatcen çuçun; arsagna lokhartia çuçun: iharsatciakin, aperceitu cicin Tantugou oïhenari buz ihessari yueiten cela. Essaoutia içan çuçun ere arçagnez menditarte pouillit batetan, harrokaten gagnen etçana.

Egoungo egounien ere, haourrac hantçat beldur tutçu, Pyrénéétako çoumbeit herrietan. Mouthiko gnimigno edo nezkatila gninignoatek bere amari sinhetsi nahi ez dienien, erreiten dakoçu: Khassu eintsak hiri! oihu eiten diat Tantugouri eta, istantien, yin douc oyhenetic!

S. Daguerre, 31 ans, né à Bardos. 


\section{La légende de Barbazan}

Béhin batéz bacirén Pirénéétaco hérri batéan guiçon bat éta émazteki bat arras çaharrac. Etçoutén étché ttipi bat, baratcé bat éta béhi bat baïcic. Baïnan, cémbat nahi béharrac içanac gatic, lagountcén citouztén bérac baïno béharragocoac. Égoun batéz, lourra élhouréz éstalia cén, éta harrién arrailttcéco horma hari cén. Hérrico yéndé gouciac hari cirén bérotcén, yatén eta édatén çoutélaric; abérats éta dohatsou cirén. Ilhoun nabaréan bi piaïant hourroundic héldou cirénac guélditou nahi içan cirén lékhou hartan, cérén hotz éta gossé baitcirén; yo çoutén athé batéan, guéro bértcé batéan éta bértcé batçouétan oraïno: nihorc étcitouén outzi nahi sartcérat, éz éta déoussic éman nahi yatérat. Aldé gouciétaric haïzçatouac, çakhour borthitcénéz houïatouac, bi arrotcéc étçakitén norat yoan, noïz éta éré guérthatou baïtcirén hérriarén baztéréan, guiçon éta émazteki béharrac cirén hékien étché aïntcinean. Aditcéarekin hékien dolhaménac, sénharra éman cen leïhoan hékien déitceco, éta harén émaztéa yoan cén laster athéaren idékitcérat. Galdétou gabé nor cirén, sar araci citouztén éta yar araci su ondoan; guéro éman ciozcatén esnéa eta cémbatï gaztaïna, citouztén gouciac. Baïnan ordouan, Gouré Yaouna - houra cén Yondoni Pétriékin- choutitou cèn bouroua argui-arguia, éta érran ciotén: "Çouéc béharrac éta onac çarété; çouen haouçoac abératsac éta gaïstoac diré. Yincoarén çoucéntassouna éguin bédi!". Haïnbértcénarékin lourra ikharatou cén, Yéssous éta Yondoni Pétri sountsitou cirén éta bi çaharrac bélhaounico érrori... Biharamoun goïcéan, hérrirat yoan nahi içan çoutélaric, yakitéco cér guérthatou cén, étçoutén étché bat ikhousi; hékien lekhouan bacén ourlo bat, oraïno égounco égounéan Barbazanéco hériarén éta Saint-Bertrand-deCommingeseco hiriarén artéan caoussitcén déna. Lagoundou béhar diré érroméssac.

Pour prononcer ce morceau et le suivant comme ils doivent être prononcés, les lire tels qu'ils sont écrits. En outre, ne pas oublier que: ler. $h$ est toujours aspiré; $2^{e} \cdot g=\underline{d}$ mouillé; $3^{\mathrm{e}}$. $\underline{t}=\underline{t}$ mouillé; $4^{\mathrm{e}}$. $z=c ̧$; $s$ doit être prononcé gras, comme le font les Espagnols; $\sigma^{\mathrm{e}}$. les voyelles nasales an, en, in, on doivent être prononcées comme en espagnol; pour cela, faire bien entendre $a, e ́, i, o$, et faire sentir le $n$ en appuyant légèrement le bout de la langue sur les incisives supérieures.

\section{La légende de Tantugou}

Tantugou da guiçon çahar bat oïhanétan gordétcén déna. Çaïntcén ditou landac éta sorhoac han éz déçatén déoussic ébats. Oguiac ébakiac dirélaric éta bélharra moztoua, naoussiac dénac bildou artéan, Tantugou éz da lokhartcén; béthi érné dago. Harén aguértcéco béldourrac lotsatcén ditou ohoïnac. Ona da, sinhets çaçou, baïnan bassa da. Laborari haïnitz gaouça çakién batéc érraïten çouen ardoura béré sémééri Tantugou céla éguiazki lourréco citouén çaintçaléric hobéréna.

Ouztaril arats aldé batéz, arthaldé bat alhan çagon méndi batén gaïnéan; artçaïna lokhartu cén; atçartcéarékin, ikhoussi çouén Tantugou ihéssi çohala oỉhanérat. Artçainétaz oraïno éçagoutoua içan cén célhai pouillit batéan, harroca batén gaïnéan étçana. 
Oraino égounco égounéan, Pirénéétaco cémbait hérriétan, haourac béldour diré haréntçat. Mothico ttipi batéc édo néchca ttipi batéc éz douélaric amarén érrana éguin nahi, érraïten dio: "Içan béldour! Déitcén dout Tantugou, éta béréhala éthorrico da oïhanétic.

Instituteur: M. Beignatborde, 33 ans, né à Roquiague (Basses-Pyrénées).

Collaborateurs: Duronto, Haran, Becas. 


\section{La légende de Barbazan}

Basiren léhénago Pyrénéétaco héri batian guison bat éta émastéki bat bisiki saharac. Etsuten etché tipi bat, baratsé éta béhi bat baïsic. Baïnan, sonbat nahi pobré isanic han éré, laguntsén sitousten béthi bérac baïno pobriago sirénac. Egun batés loura élhourès éstalia sen, éta horma garatsac ari siren. Hérico yenté gousiac, jan éta édan, bérotsen hari siren; abérats éta ourous siren. Ilhoun gnabarian, bi bidéyant, hourounétic héldou sirénac, nahi isan siren guélditou toki hartan, ésen gosé eta égari siren; jo souten bortha batian, guéro bertsé batian éta bertsé ascotan oraïno: nihorc étsitouen outsi nahi isan sartséa, és éta éman nahi isan déousic jatéra. Aldé gousiétaric casatouyac, chakhour gachtouès séguitouyac, bi bidéyantéc étsakiten norat joan, noïs éta éré khaousitou béitsiren héri bastérian, guison éta émastéki pobré héyen etciaren aitsinian. Héyen plaïniac aïtsiarékin, sénhara éman sen léihoan héyen déitséco, éta émastia joan sen fité bortharen idékitséra. Galdéguin gabé nor siren, sar arasi éta sitousten eta jararasi souphastérian, guéro, éman sacoten esné éta gastaina sonbait jatéra, souten gousia. Baïnan ordouan, Jésou-Christo, - ésen houra éta Jondoné Phétiri siren- choutitou sen, bourou gousia dirdiran, éta éran sacoten: "Souec, pobriac siésté baïnan onac; souen aousouac abératsac diré, baïnan gachtouac, Jincouaren jousticia éguin dadiéla!”. Hanbesténarékin, loura ikhratou sen, Jésou-Christo éta Jondoné Phétiri galdou siren, éta bi saharac éman siren bélhaounka... Biharamen goïsian, joan nahisan sirénian hérirat, jakitéco sen pasatou sen, étsouten étché bihi bat ikhousi: héyen tokian, poutsou handibat sen,

Oray oraino khaousitsen dena Barbasanéco hériaren éta Saint-Bertrand-deCommingéco hiriaren artian. Lagoundou béhar dira béharétan dirénac.

\section{La légende de Tantugou}

Tantugou da guison sahar bat oyhanétan gordatsen déna. Saïntsen tou landac éta pentsiac nihorc déousic ébats és désan. Oguiac édo bélharac pikatiac dirénéan, gousiac naousiac sar artio, Tantugouc ésdou loïc éguiten, béthi béilan dago. Haren aguer béldourac, saïntsen tou ohoïnac. Ona da, sinhets sasoué, baïnan salbaya. Hanits gaousa sakien laborari batéc éraïten souen maïs béré sémér Tantugou séla ségourki louréco frouitouen saintsaléric hobéréna.

Ustaillaco aratsaldé batés, arthaldébat alha sagouen méndi batian; artsaina lo sen: iratsartsiarékin ikhousi souen Tantugou ihési jouan oyhanérat bourous. Guisa bérian ésagoutia isan sen artsaïnéz, haroka batian étsana, érépia poillit batian.

Egoun oraïno, Pyrénéétaco héri batsouétan, haourac haren beldour dira; Noïs éta éré motico édo néscatcha batéc esbeitouté amari obéditou nahi, éraitten dacoté: Gouardia! déithouco diat Tantugou éta braou jinen douc oyhanetic!

Instituteur: Etcheberrigaray,

âgé de 33 ans, né à Alos (canton de Tardets)

Coll.: Hardoy (Jeanne), Celhay (Félicie) 


\section{La légende de Barbazan.}

Bacen demboras gure mendietako herri batian guiçon eta emastekibat arras chaharrak. Ez çuten etche ttippi bat, baratce bat eta behibat baicik. Bainan, nahis hain erromesak ciren, laguntcen citusten berak baino erromesagoak. Egun bates, lurra elhurres estalia cen eta horma haicen harriak arrailtceraino. Herriko yende guciak berotcen haiciren yaten eta edaten çutelarik. Aberats eta urus ciren. Ilhuntcian, bi piayant, urrundik heldu cirenak, nahi içan çuten herri hartan guelditu hots eta gose çutelakots. Yotcen dute ate batian, guero bertcebatian eta oraino bertce batçuetan. Nihork ez cituen sartcera utci nahi es eta ere yatera deusik eman nahi. Gucietarik kasatuak, chakur gaichto batçues seguituak, bi arrots horiek ez çakiten norat yuan, nois eta ere kausitcen baitira, herriaren basterbatian, guiçon eta emaste erromes horien etchiaren aintcinian. Aditcearekin arrots hoyen arrangurak, senarra emantcen leyoan heyen deitceko eta emastia yuantcen atiaren idekitcea. Galdatu gabe nor ciren, sarraraci eta yarraraci citusten beren supasterrian, guero eman cioten esnia eta çombeit gastena, çuten gucia. Bainan orduan gure Yésukhrichto yaunak -hura cen yondoni Petriikin- chutitcen da burua distirantik eta erran cioten: çuik erromesak eta onak cieste; çuien hausoak aberatsak eta gaichtoak. Yainkoaren yusticia eguin daila! Berehala lurra ikaratcen da, Yésus eta yondoni Petri suntcitcen eta bi çaharrak belhauniko erortcen. Bihamun goician, yuan nahi içan çuten herrira yakiteko cer pasatcen cen, es çuten etche bakarbat ikusi: hauyen lekuyan bacen ur çabalbat, çoina kausten baita oraino egungo egunian Barbazango herriaren eta san Bertrand-de-Comminges eko hiriaren artian.

Bethi lagundu behar dira beharrak.

\section{La légende de Tantugou}

Tantugou da guiçon çahar bat gordetcen dena oyanetan. Çaintcentu landak eta pentciak deusik ebats ez deçaten. Nois eta oguiak pikatuyak edo pentciak segatuyak baitira eta gauça hauk nausiak etchian sartu arte Tantugouk ez du lorik eguiten. Bethi çain dago. Hunen ikusteko beldurrak guibelatcentu ohoinak. Ona da, sinhets çaçu, bainan basa da. Laborari hainits gauça çakin batek erraiten cioten maïs bere semeri Tantugou cela segurki lurresko gaucen çaintceleik hobena. Ustail hilabeteko arratsaldebates, ardi multsu bat ala cen mendibaten gainian, artçaina lokartucen: iratçartciarekin ohartu cen Tantugou ihes çohala oyaneko aldera. Bertce artçain batçuek ere eçagutu çuten erreka celai eder batian etçana harroka baten gainian.

Egungo egunian oraino, gure mendietako herri batçuetan haurrek haren beldur dute. Nois eta mutiko edo nechkato ttippi batek obeditu nahi ez duinian bere amari erraiten dio: Kasu eiçak! Deitcendiat Tantugou eta laster yenenduk oyanetik. 


\section{La légende de Barbazan / Barbazen-en Omena}

Baciren bertce orduz Pyrenees-taco herri batean guiçon bat eta emazte bat cirenac arraz çaharrac. Ez çuten etche tippi bat, baratce bat eta behi bat baicic. Bainan, çombat pobre içana gatic, soccorritcen cituzten bethi berac baino pobreago cirenac. Egun batez, lurra estalia cen elhurrez eta horma harrien erdiratcecoa hari çuen. Herrico yende guciac, berotcen çauden, yaten eta edaten hari citelaric, aberatz eta uruz ciren. Ilhuntcean, bi bideyante urrundic heldu cirenac, nahi içan çuten arrastatu leku hortan ceren eta hotçac eta goseac baiçauden; yo çuten bortha batean, guero bertce batean, eta bertce çombeitetan oraino: Nehorc ez cituen nahi içatu sartcerat, ez eta ere deus eman yateco. Cassatuac gucietaric, perseguituac sakhur hassarretu batçuez, bi estranyerac ez çakiten norat yoan, noiz eta ere aurkhitu baiciren, herriaren buruan, pobre ciren guiçon eta emazte heyen etchearen aintcinean. Entçutearekin heyen plainuyac, senharra eman cen leyhoan deitceco, eta haren emaztea yuan cen fite bortharen idekitcerat. Galdeguin gabe nor ciren sarraraci eta yarraraci cituzten beren su pasterrean; guero cerbitcatu cioten esnea eta çoimbeit gastena; çuten gucia. Bainan orduan, Gure Jauna -ecen hura bera cen Jondoni Petrirekin- alchatu cen, buruya gucia distiratua, eta erran cioten: "Çuec pobreac eta onac cirete, çuen hauçoac aberatsac eta gachtoac dire. Jaincoaren yusticia eguina içan dadiela!". Ordu berean lurra ikharatu cen Jésus et Jondoni Petri suntsitu ciren eta bi çaharrac belhaunico erori ciren... Biharamun goicean, yuan nahi içatu cirenean herrirat yakiteco cer passatu cen, ez çuten ikhusi etche bat bakharra: heyen lekhuan bacen oussi bat, çoina aurkhitcen baita oraino egun Barbazango herriaren eta San-Bertran-de-Comminges-co hiriaren artean. Behar dire lagundu erromesac.

\section{La légende de Tantugou / Tantugou-ren Omena}

Tantugou guiçon çahar bat cen gordetcen cena oyhanetan. Çaintcen ditu landac eta pentciac nehorc deus ebats ez deçan. Oguiac edo belharrac picatuac direnean, nagussiac guciac sarthu dituen artean, Tantugouc ez du loric eguiten; bethi beilatcen du. Hura arguetceco beldurrac urruntcen ditu ohoinac. Ona da, sinets çaçu, bainan salbaya da. Laborari batec gauça hainitz çakienac, erraiten çuen maiz bere semei Tantugou cela segurki lurreco fruituen çaintçaleric hoberena.

Uztaileco arratçalde batez, ardi tropa bat allatcen cen mendi baten gainean; artçaina lokartu cen; atçartcean, ohartu cen Tantugou ihez çohala oyhan alderat. Eçagutua içan cen higual bertce artçain batçuez haran pullit batean, etçana harroca baten gainean.

Oraino egun Pyrenées-taco cembeit herritan haurrac haren bedur dire. Noiz eta here mutiko tippi edo nechca tippi batec ez baitu obeditu nahi bere ama, erraiten dio: "Emaçue casu! Deitcen dut Tantugou eta mement berean, yenen da oyhanetic."

Instituteur: M. Suhas, âgé de 47 ans né à Guiche Coll.: Teillery 


\section{La légende de Barbazan / Historioa Barbazanecoua}

Lehengo denboretan basen heri batean gison eta emasteki chahar bat, ez souten etche tipi, baratce eta behi bat baisik. Nahiz bethi lagountsen sitousten hek baino pobreagoac sirenac. Egoun bates loura estalia sen elhoures eta horma handia hari. Bi pirayant ouroundik heldou sirenac, nahi isan siren arastatou lekou hartan, seren hotz eta gose baitsiren, borta bat yotsen doute, gero bertse bat, bainan nehorc ez ditout barnerat sararasten, eta ez yaterat escaintsen orotarik kasatouac, etche hetaco sakourek aintsinean erematen ditouste, bi pirayant hoic soin ez baitsakiten norat yuan, heri haren bertse kantoïnean sirelarik, gison eta emasteki pobre haren etchearen aintsinean gison eta emasteki chahar hauyek aditsen doute hauyen plainouac, eta gison chahar hau yartsen da leihouan eta oihou eguiten dioté, dembora berian emasteki chahar harek idokitsen diote borta, galdatou gabe nor siren, yar arasten ditouste eta emaiten diote yaterat esnea eta gastena ditoustenac.

Bainan ordouan goure Jaincoa (soin pirayant hetarik bat houra baitsen Yuandoni Petrirekin) choutitsen da, distiratsen douelarik eta eraiten diote souek pobriac siste eta onac, souen hausoac aberatsac eta goustouac. Jaincoaren yusticia eguina isan dadiela, dembora berian loura ikaratsen da Yesous eta Yondoni Petri yuaiten dira eta bi yende chahar hauc belhaunico erortsen dira.

Biharamoun goisean yuan isan siren carikara yakitera ser pasatou sen, ez doute ikousten etcherik batere, etchien plaçan our handi bat sen, soin orai ere our houra pasatsen baita Barbazaneren eta San-Bertrand-Comingeko hiriaren artetik.

Beras behar dira pobreac lagoundou.

\section{La légende de Tantugou / Tantugouren istorioa}

Tantugou gison sahar bat da gordetsen dena oihanetan. Seintsen ditou landa eta pentsiac. Oguiac eta belharac pigatouac direnean ez dou loric eguiten, hec nausiac barnerat eman artio. Ohoinac ez dire hourbiltsen haren beldoures. Sinhets sasoue gison ona da, bainan salbaya. Gison laborari hanitz gausa sakien batek eraiten cioten bere semeeri segour Tantugo kasoueguilerik hoberena dela, loureco fruitouentzat.

Oustaileco aratsalde bates, ardi tropa bat pasatsen sen mendi batean, artsaina lokartu sen eta iratsartsen delarik, ikousten dou Tantugo yohan dela oihaneco aldera, eta gisa berian ikousi isan souen bertse artsain batek, etsana sagola, hari mendi baten pountan.

Oraico egounean mendietaco heri hauetan haurac hanitz beldour dira haren, neska edo mouthico tipi sombeitek ez doutenian nahi obeditou beren amari, eraiten diote kasou eguisan Tantugori oihou eguinen dionat eta oihanetik yinen douk fite.

Instituteur: Adrien Larrieu-Let, 3lans, né à Monein Coll.: Pour la traduction, ma femme, qui est basquaise. 


\section{La légende de Barbazan / Barbazan deitzen den herriko errankizuna}

Lehengo demboretan, Pyrénées-mendietako baster-herri batean, baziren gizon bat eta emazteki bat arras zaharrak. Ez zuten berenik etche ttiki bat, baratze bat eta behi bat baizen. Bainan, zembat nahi pobre ziren, bethi laguntzen zituzten hek baino pobreago zirenak. Egun batez, lurra elhurrez estalia zen eta horma izotzarekin hari zuen. Herriko yende guziak berotzen hari ziren, yaten eta edaten zutelarik; aberaxak ziren eta dohaxuak. Ilhun-nabarrean bi bidelari, urrundik heldu zirenek, nahi izan ziren gelditu lekhu hartan, hotz zutelakotz et gose zirelakotz; yo zuten athe batean, gero bertzean eta oraino bertzetan ere; nihork ez zituen utzi nahi izan sartzera ez eta ere deus eman nahi izan yatera. Lekhu guzietarik egorriak, chakur gaiztoez persegituak, bi gizon arrotz hoyek ez zakiten norat yo, noiz eta ere kausitu baitziren herriaren buru-buruan, gizon eta emazteki pobre heyen etchearen aitzinean. Bidelarien auhenak aditzearekin, senhara eman zen leyhoan heyen deitzeco eta emaztea guan zen berehala athearen idekitzera.

Galdegin gabe nor ziren, sar-arazi zituzten eta yar-arazi su-bazterrean; gero eman ziozkaten esnea eta zembait gaztaina, zuten guzia. Bainan orduan Jesu-Kristo gure yauna ezen hura zen yondoni Petrirekin- altchatu zen bisaia distirant batekin eta erran zioten: "Zuek pobreak zarete eta onak! Zuen hauzoak aberaxak dire eta gaiztoak. Yainkoaren yustizia egin dadila!...". Ordu berean, lurra ikharatu zen, Yesus eta Yondoni Petri guan ziren biztatik eta bi senhar emazte zaharrak erori ziren belhauniko.....

Biharamun goizean, guan nahi izan zutenean herrira yakiteko han zer iragan zen, ez zuten ikhusi etcherik batere; etcheen lekhuan bazen ur zabal bat, zeina han kausitzen baita oraino egungo egunean ere, Barbazaneko herriaren eta Saint-Bertrand-de-Comminges deitzen den hiriaren artean. Behar dire lagundu dohakabeak.

\section{La légende de Tantugou / Tantugou-ren errankizuna}

Tantugou da gizon chahar bat oihanetan gordetsen dena. Zaintzen ditu landak eta sorhoak, han deusik ebax ez dezaten. Ogiak eta belharrak pikatu izan direnean, nagusiak etchean sarthu ditueneraino, Tantugouk ez du lorik egiten; bethi erne dago. Haren agertzen ikhusteko beldurrak geldi-arazten ditu ohoinak. Ona da Tantugou, sinhets nezazue, bainan basascoa da. Hainitz gauza bazakien laborari batek erraten zioten maiz bere semeeri Tantugou zela segurki lurreko ontasunen zaintzailerik hoberena.

Uztaileko arrax-alde batez, arthalde bat alhan zen mendi baten gainean; artzaina lokhartu zen; atzartzean ikhusi zuen Tantugou ihesi zohala oihaneco aldera. Ezagutua izan zen ere behi zainzalez haran eder batean, harroska baten gainean etzana.

Oraino egungo egunean, Pyrénées-mendietako bazter herri batzuetan, haurac Tantugouren beldur dire. Muthiko edo nechka ttiki bat amaren erranari yarreikitzen ez delarik hunek erraiten dio: Kasu egik! Oihu egiten zioyat Tantugoui eta berehala ethorriko duk oihanetik

Instituteur: Amirint, âgé de 30 ans, né à Idaux-Mendy, B. P., Coll.: M. M. Elissamburu et Leremboure 
La légende de Barbazan / Barbasango ichtoriua

Bazien leheño (lehenago) Pirenetaco herri batian gizon bat eta emazteki bat arras zaharrak zieñak (zirenak). Etzuten etche ttipi bat, baâtze (baratze) bat eta behi bat baizik. Bainan hain pobriak izanik ê (ere), sokorritzen ziuzten bethi hek baino pobriô (pobriago) zienak. Egun bathez, lurra estalia zen elhurrez eta horma haizuin karraskan. Herriko yende guziak berotzen zien yaten eta edaten haizielaik (harizirelarik); aberatsak eta urusak zien. Iluntchian, bi bidelari, helduzienak urrhundik, nahi izantzien gelditu toki hartan zeên (zeren) hotz eta gose baitzien; yozuten athe batian, gero bertze batian eta bertzetan oîno (oraino): niok etziuzten nahi izan utzi sartzerat ez eta eman nahi izan yaterat. Kasatuyak orotaîk (orotarik), lasterkatuyak zakur tzarrez, bi estranyerek etzakiten noât (norat) yohan arribatu zienian herriko bertze puntarat, emazteki eta gizon pobriak zien heyen athe aintzineât (aintzinerat). Aitziarekin heyen pleinuyak, senarra yarrizen leyuan heyer oyhu egiteko, eta haren emastia yuantzen fite athiaren idekitzeât (idekitzerat). Galdegin gabe nortzien, sarrâzi (sarrarazi) ziuzten eta yarrâzi (yarraazi) been (beren) supazterrian; gero zerbitzatu zioten esnia eta zombeit gastena, ziusten guziak. Bainan orduyan, Gure Yinko Yauna, -hura zen Yondoni Petirikin, - yekizen, buru guziak dirdiâtzen (dirdiratzen) ziola, eta erranzioten: "Zuik, pobriak eta onak zizte; zuin hauzuak aberatsak eta tzarrak. Yinkoaren yustizia egin dadiela." Berehala lurra ikaratuzen, Yesus eta Yondoni Petiri suntsitu ziên (ziren) eta bi zaharrak belhauniko erorizien... Bihâmun (biharamun) goizian nahi izantzutenian yuan herrirat, yakiteko zê (zer) pasatuzen, etzuten ikusi etche bihik (bihirik): heyen tokian zen ur haundibat, oîno (oraino) egungo egunian Barbasango herriaren eta Yondoni-Petan-de-Commingesgo hiriaên (hiriaren) artian dena.

Behardia lagundu malurusak.

$$
\text { oOo }
$$

\section{La légende de Tantugou / Tantuguin ichtoriua}

Tantugu zahar bat da gordetzen dena oyhanetan. Zaintzentu landak eta sorruak ez dezaten deusik ebats. Ogiak eta belharrak pikatuak dienian, oro altchatuyak izan artio nausiaz, Tantuguk ez du loîk (lorik) îten (eguiten); bethi zaindô (zaindago). Haên (haren) ikusteko beldurrak atchikitzentu ohoinak. Onada, sinetsazu (sinets zazu), bainan salbayada. Laborari batek, bazakienak hainitz gauza, ertentzuin (erraitentzuin) maiz bere semier Tantugu zela seûrki (segurki) lurreko fruituyen zaintzaleik (zaintzalerik) hobêna (hoberena).

Uztaileko âtsalde batez (arratsalde), ardi tropa bat alatzenzen mendi batian; artzaina lokartuzen: iatzartzian ikusizuin Tantugu espekatzenzela oyhaneko aldeât (alderat). Izantzenê (izantzen ere) ezaûtuya (ezagutuya) behi zaintzaliez erreka pullit batian, etzana harroka baten gainian.

Oîno (oraino) egungo egunian, Pirenetaco zombeit herritan, haurrak haentzat 
(harentzat) beldur dia (dira). Mutiko edo nechka ttipi batek ezduenian obeitu (obeditu) nahi bere amari, erten (erraiten) diote: Kasu izak, oyhu itendiat Tantugui (Tantuguri), eta behâla (berehala) yinenduk oyhanetik.

Instituteur: P. Bétachet, 26 ans, né à Espelette Coll. Elissamburu, Bessonnart

Remarques sur les deux légendes

De même qu'en français à pierre fendre: Karraskan est une expression consacrée qui traduite littéralement veut dire avec crâquement.

Aujourd'hui: egungo egunian, expression consacrée; littéralement au jour d'aujourd'hui.

Autres remarques

Le labourdin sobre de paroles prononce d'une façon et écrit d'une autre. Dans les deux traductions j'ai tâché d'indiquer entre parenthèses les mots qui, prononcés brièvement avec omission d'une ou de plusieurs lettres, sont écrits avec toutes leurs lettres par les mêmes personnes.

Ainsi on prononce lehenô en appuyant un peu sur la dernière lettre et la même personne écrira, sans s'en douter: lehenago

De même, pour: ziênac; zirenak, - baatze, baratze, etc. etc...

Prononciation

Voyelles: $a$ de même qu'en français; - $e$ toujours é; $-i$ : $i$; -o: $o$; - $u$ toujours ou.

Consonnes: $b, t, f, h, l, m, n, p, t, v$ se prononcent toujours comme en français.

- $c$ ne s'emploie que dans ch, comme chien: chakurra

- g a toujours la prononciation dure de guerre: gerla

- j ne s'emploie jamais

- $k$ toujours dur; s'emploie au lieu de c: karraskan

- $q$ ne s'emploie jamais

- $r$ toujours dur se double dans le corps d'un mot et simple à la fin. Exemple; gezurra (mensonge); bihar (demain)

- $s$ a toujours une prononciation se rapprochant de $j$ et de $z$ français

- $x$ ne s'emploie jamais

- $z$ a toujours la prononciation de ç

- N.B. - la voyelle y se prononce toujours comme dans moyen; yauna (monsieur) 


\section{La légende de Barbazan / Barbazaneco historia}

Leheneco mendetan, Pyrenesetaco herri batian baciren gizon bat eta emazteki bat biciki çaharrac cirenac. Etche nimino bat, baratze bat eta behi bat hec ciren heien ontasunac. Hainitz probriac ciren bainan bethi laguntcen ciusten berac baino probiagoac. Egun batez elhurrez estalia cen lurra, horma bat cen hain handia noun harriac ere hausten baicituen. Herriko yende guziac berotcen hari ciren yatian eta edatian hec ciren aberatsac eta urusak. Ilhuntzian, bi piaiant urrundanic heldou cirenac nahi izan cuten leku hartan guelditu ceren eta hots eta gose baitciren yo çuten borta bat, yo çuten bertce bat eta bertce cembait oraino: nehorc ez cituzten sartu nahi ukan ez eta yaterat eman nahi. Leku guzietaric casatuac, çakur guciez seguituac, bi arrotzec ez çakiten norat yuan noïz eta ere causitu baitciren herriaren bertce buruan gizon eta emazteki probe heien etchearen aïtcinean. Piaïant heyen pleinuac aditcearekin senharra atheratu cen leyherat oyhu eguiteco eta emaztea berehala yuan cen bortaren idekitcerat. Arrotz heyer galdatu gabe nor ciren sarraraci cituzten eta subasterrian yarraraci eta eman çacoten yatea cituztenetarik: esnia gaztena cembaitekin. Bainan orduan goure Yainco Yaunac hura bera cen Yondoni Petirikin, chutitu cen buru dirdirant batekin eta erran çuen yende probe heyer: "Zuec probiac bainan onac cirezte; zuen aüzoak aldiz aberatsac becen gaistuak siren. Egina izan bedi Yaincoaren yusticia!". Bet betan lurra ikaratu cen, Yesus eta Yondoni Petiri ezestatu ciren eta gizon eta emasteki çahar probe hec erori ciren belhaünico... Biharamun goizian yan nahi izan çuten herrirat yakitera zer pasatu cen, ez çuten ikusi etcheric bihic: heien plazan bacen ur tulumbi bat zoïna egun oraino ikusten baita Barbazan deitzen den herriaren eta St Bertrand de Comminges deïtzen den hiriaren artean. Malurusac behar dire lagundu.

\section{La légende de Tantugou}

Tantugou gizon çaharbat da çoina oyhanetan gordetzen baita. Landac eta phenciac zaintzen ditu nehorc ez dezan phitsic ebats. Ogiac eta belharrac picatuac direnian, Tantugouc ez du loric eguiten hec guciac sartu artino; bethi beilan dago. Ohoïnac daude heren ikustearen beldurrez. Salbaia da bainan perestua sihesten ahal nuçu. Laborari batek çoinac hainitz gaûzen gainian arguitua cena erraiten çuen usu bere semier Tantugou cela dudarik gabe lurreko fruituen zaintzale hoberena.

Uztailaren arratsalde batez, artalde bat alhan zagon mendi batian; artzaina lokartu cen: atzartzearekin, ikusi çuen Tantugou ihes eguiten oyhanari buruz. Abere zaintzale batçuec ere mendi arte puillit batean kausitu çuten Tantugou hura arroca baten gainian etzana.

Oraico egunetan ere, Pyrenesetako zembait herritan haurrac haren beldur dire. Mutico ttipi bat edo neskato ttipi bat guerthatcen denian obeditu nahi ez duenac, amac erraiten dio: Casu izak! oyhu eguiten diat Tantugouri, eta, orai berian yinen duc oyhanetic!

Instituteur: Loustalet, 34 ans, Lourdios-Ichère Coll.: Etcheberry, Pierre 
Behin baciren Pyrenesetako herri gnimigno batian gizon bat eta emazteki bat arras çaharrac. Etche gnimigno bat, bahatze bat eta behi bat çuten ontasun guzitzat. Hanitz pobriae beharrian ciren, beïnan bethi laguntzen cituzten hec baïno pobriagoae beharragoac. Egun batez, lurra elhurrez estalia cen, horma hanitz handia ere cen. Herri hartaco yende guziac berotzen hari ciren, yatian eta edatian, aberatsak eta urusak ciren. Ilhuntziarekin, bi gizon, urrundic heldu cirenac, nahi izan çuten arrastatt gelditu leku hartan, ceren hotz eta gose baïtciren. Yo çuten ate bat, gero bertze bat eta oraïno bertze batzuc; nehoc ez cituzten sartzerat utzi nahi izan, eta nehoc ere ez cioten yaterat eman nahi izan. Leku gucietarik casatuak, çakur errabiatu batzuez bezala segituak, bi estranyer arrotz heyek ez çakiten norat yuan, noïz eta ere kausitu baitciren herri haren bertze buruan, gizon eta emazteki pobre beharrian ciren heyen etchearen aïtzinean. Estranyer Arrotz heyen pleïnuac aditzearekin, senarra emantzen leyhoan oyhu egiteko eta emaztea yuan cen fite laster bortaren atearen idekitzerat. Estranyer Arrotz heyer galdatu gabe nor ciren sar hazi cituzten eta su bazterrean yar azi; gero zerbitzatu eman cioten etchean cituzten yateco guziac: esnia eta zonbait gaztena. Bainan orduan, Yainko yaunac - ceren hura baitcen yondoni Petirikin - chutitu cen, burua dirdiran zuelarik, eta erran zuen yende pobre behar heyer: "Zuek pobriae beharrac eta onac zaïzte, zuen haüzoak aberatsac eta gachtoac dire, Yainkoaren yustizia eina izan bedi!". Orduan, bet betan, lurra ikaratu zen. Yesus eta Yondoni Petiri esestatu ciren eta gizon eta emazteki pobre behar hec erori ciren belhaünico... Bihamun goïzian yoan nahi izan çuten herrirat, yakiteco zer pasatt gertatu cen, ez çuten ikusi etcheric batere; etchen plazan lekuan cen ur handi bat, zoïna ikusten baita oraïno Barbazan deïtzen den herriaren eta Saint-Bertrand-de-Comminges deïtzen den hiriaren artean.

Malhurusac Beharrian direnac behar dire lagundu.

oOo

Tantugou da gizon çahar bat zoïna gordetzen baïta oyhanetan. Zaïntzen ditu pentziak, nehoc ez dezan phitsic ebats. Ogiac eta belharrac pikatuac direnian, Tantugouc ez du loric eguiten ogi hek eta belhar hek sartu artio, bethi beilan dago. Ohoïnak hartaz arras beldur dire. Pestua da, sinhets zazue, bainan salbay basa da. Laborari batec, zoïnak baitzazkin hanitz gaüza, erraten zuen maïz bere semeri Tantugou zela segurki lurreko fruituen zaïntzale hobena.

Uztaïlaren egun arratsalde batez, arthalde bat paüchan zagon mendian, artzaïna lokartu cen: atzartziarekin ikusi zuen Tantugou eskapatzen lasterka yoan zela oyhaneko alderat. Ezagutua izan ere cen abere zaïntzale batzuez, mendi arte puillit batean, harroka baten gainean etzana.

Orainoko egunetan ere, Pyrenesetako zombeit herrietan, harrae haürrac hartaz beldur dire. Noïz eta ere mutiko gnimigno batec edo nechka gnimigno batek bere amari obeditu nahi ez duenean, amak erraiten dio: Intzac casu! Oyhu egiten diat Tantugouri eta oraï berean yinenduk oyhanetic! 
Inst.: G. Guilliard, né à Espelette, le 23 septembre 1861.

Nota. - Tous les u se prononcent ou. - Tous les e se prononcent é. - ge gi se prononcent gué gui. - en em se prononcent in. - in se prononcent ine. - s se prononce en appuyant le bout de la langue contre le palais et en sifflant. - z se prononce comme l's en français. - un se prononce oun. 
Baciren bertce orduz Pyreneco herri batean guiçon bat eta emaste bat biciki çaharrac cirenac. Ez çuten etche ttipi bat, baratce bat eta behi bat baicic. Bainan, cembat nahi pobre içanagatikan, sokoritcen cituzten bethi berac baino pobriago cirenac. Egun batez, lurra estalia cen elhurrez eta horma ari cen bortiski. Herrico yende guciac berotcen ari ciren, yanez eta edanez; aberats eta urus ciren. Ilhuncian bi pidayant heldi cirenac urrundic nahi içan ciren guelditu lekhu hartan, ceren hots eta gose baitciren; yo çuten bortha batian, guero bertce batian eta bertcetan oraino: nihorc ez cituen utci nahi içan sartcera ez deussic eman nahi içan yatera. Kassatuyac orotaric, seguituyac sakhur errabiatyuez, bi estranyerec ez çakiten norat yoan, nois eta ere khausitu baitciren herri basterrian, guiçon eta emaste pobre heyen etchiaren aitcinian. Aditciarekin heyen plainuyac senharra eman cen leyhoan heyen deitceco eta haren emastia yoan cen fite bortharen idekitzera. Galdatu gabe nor ciren, sar eraci ciusten eta yar eraci beren su basterian, guero cerbitçatu çakoten esnia eta cembait gastaina, hec ciren citusten guciac. Bainan orduyan, Yesu-Christo gure Yauna, - hura cen Yondoni Betirirekin, - chutitu cen, buruya dena distiretan eta erran çakoten: "Çuek, pobriac eta onak çarete; çuen aussoac aberatsac eta gaistoac dire. Yaincoaren yusticia eguina içan dadiela!". Berehala lurra ikharatu cen, Yesus eta Yondoni Betiri bistatic galdu ciren eta bi çaharrac erori ciren belhaunico... Biharamun goician, nahi içan çutenian yuan herrirat, yakitera cer passatu cen, ez çuten ikhussi etcheric batere: heyen plaçan bacen ur gueldi bat edo aintzira bat, hura bera khausitcen dena oraino egun Barbazaneco herriaren eta San Bertrand-de-Commingeco hiriaren artian. Behar dire sokhoritu malurusac.

(Observation: le $u$ se prononce ou)

oOo

Tantugou da guiçon bat gordetcen dena oyhanetan. Çaincen ditu landac eta phenciac ez deçaten deussic ebatx. Oguiac phicatuyac edo belharra ebakia direnian, denac sarthuyac içan artian naussiaz, Tantugou ez da batere loac hartcen, beilatcen du bethi. Haren aguertcen ikhusteco beldurrac atchikitcen ditu ohoinak. Ona da, sinhetx çaçue, bainan salbaya da. Laborari batec, çoinac baitçakien hainits gauça, erraiten çuen mais bere semeri Tantugou cela segurki lurreco fruituen sainçaleric hoberena.

Ustaril hilabeteco arraxaldi batez, ardi arthalde bat alhatcen ari cen mendi baten gainean, artçaina loak hartu çuen; iratçartcian ikhussi çuen Tantugou oyhanerat ihessi çohala. Higual eçagutia içan cen artçainez mendiarte poillit atean, etçana harroka baten gainean. Oraino egun, Pyreneco hessi cembaitetan, haurrac haren beldurra badute. Nois eta ere muthico ttipi batec edo nescato ttipi batec ez baitu obeitu nahi bere amari, hunec erraiten daco: Guardi emac hiri! Deitcen diat Tantugou, eta istantian yinen duc oyhanetic!

Inst.: Saffores Jean, âgé de 46 ans, né à Tardets-Sorholus Coll.: Dunat Jean, âgé de 87 ans. 
Baziren demboraz Pirenetaco herichka batian gizon eta emazte chahar batzu. Ez zuten etche ttipi bat, baratce bat eta behi bat beïcic. Baïnan hain beharrac izanic ere laguntcen zituzten bethi berac baino beharago cirenac. Egun batez lurra elhurez estalia celaric eta harrien arditic egiteco horma hari celaric, karrikaco yende guciac berotcen hari ciren, yanez eta edanez: Aberats ciren eta urus. Ilun nabarrian bi urundic heldi ciren bidayantec nahi ukan çuten gelditu toki hartan, gose eta hotz cirelakotz. Yo zuten athe bat, gero bertce bat, eta bertcek ere oraino. Nihorc ez cituzten sartcerat utzi nahi ukan eta nihorc ez cioten yaterat eman nahikatu ere. Kasatuac bazter gucietaric, persegituac chakur arrabiatu batzuez, bi arrotcec ez zakiten norat yoan, noiz eta ere kaüsitu baïtziren karikaren buruyan, gizon eta emazte behar heyen etchiaren aïntcinian. Heïen plaïnuac aditzian, senarha pharatu cen leïhoan heïen deïtzeco eta haren emaztia yuan cen berehala athearen idekitcerat. Galde egin gabe nor ciren, sarrarazi cituzten eta yarrarazi beren su bazterrian: gero cerbitzatu cioten esnia eta gaztaïna zombeït: cituzten guciac. Baïna ordian Gure Yaunac - Hura cen Yon doni Petrirekin - chutitu cen buruya distirante eta erran cioten: "Zuec beharrac zaëte eta onac; zuen haüzoak aberatsak dire eta gaïchtoac. Yeinkoaren yusticia egin dadiela!”. Ordu berian lurra ikharatu cen, Yesus eta Yon doni Petri suntsitu ciren eta bi chaharrac belhaunka eman ciren.

Biharamun goïcean, karrikarat yuan nahi izan zutenian, cer iragan cen yakiteco, ez zuten ikusi etche bihi bat: heien tokian putçu bat cen egungo egunian oraïno Barbazaneco herriaren eta San Bernat Comintseneco hiriaren artean caüsitcen den putçu hura.

Behardire lagundu beharrac.

N.B.U se prononce OU.S se prononce S. GI se prononce gui. GE se prononce gue. Dans le vrai basque on ne met pas d'accent. Ainsi eta se prononce éta, ere se prononce ére.

\section{oOo}

Tantugou oyhanean gordetcen den chahar bat da. Zaïtzen ditu landac eta pentzeac yeusic ebatse ez dezaten. Bihi lastuac pikatuac direnian eta belharrac segatuac, guciac naüsiac bilduac izan arthe, Tantugouc ez du loïc eguiten: bethi iatzarria dago. Haren aguertceco beldurac daozka ohoïnac. Ona da, sinhets zazie, baïnan da salbaya. Labori haïnitz gaüza zakien batec erraten zuen mais bere semeri Tantugou cela segurki lur huntaco fruituen zaintzaïleric hoberena.

Uztaileco eguerdi ondo batez, ardi tropa bat alatcen cen mendi baten gaïnian; artzaïna lokartu cen: iyatzartcian ikusi çuen Tantugou oyhan alderat ihes eguiten. Ezagoutua içan cen halaber artzainez ordokhiarte batean, harroka baten gainian etzana.

Oraïnon egun, Pirenetaco zombeit herritan, haurrac haren beldur dira. Noiz eta ere mutiko ttipi edo nechka ttipi batec ez baïtu obeïtu nahi bere amari, erraten dio: kasu izac (edo izan) hiri! Deïtzen diat (edo dinat) Tantugou eta berehala yinen da oyhanetic!

M. Jean-Marcel Etcheverry, 27 ans, né à St-Pée-sur-Nivelle (B. Pyr.) Coll.: Ihistary, Jean, âgé de 90 ans. 


\section{Barbazaneco erran çaharra}

Lehenau bacien mendietaco herri batean guizon bat et emazte bat biciki çaharrac. Ez çuten etche ttipi bat, baratce bat eta behi bat baicik. Bainan hain pobreak içanik ee, bethi laüncen ciuzten berak baino pobreaüak. Eün batez lurra elhurrez estaliacen et harria arraïlceco horma aï cien. Herrico yende guciac berotcen cien yaten eta edaten ai cielaik: aberatsac eta urusak cien. Ilhuncean, bi piayant urrunetik heldu cienek lekhu hartan guelditu nahi içan çuten ceren hotzak eta goseak baitcien; yo çuten borthaat, gueo berceat eta berce batçu oono: nehok ez citien nahi utci sartcea ez eta ee eman yeusik yatea. Casatuac ootaik, yarraikiak chakhur gaichtoez bi arrotzek ez çakiten noat yoan causitu cienean herriaren basterrean guizon eta emazte pobre heen etchearen aitcinean. Heen pleinuak aitceaekin senharra eman cen leïhoan eta emaztea yuan cen fite bortharen ideitcea. Galdein gabe nor cien, sar aaci ciuzten eta yarraaci bee supasterrean; gueo cerbitçatu cacoten esnea eta combait gaztena, çuten gucia. Bainan orduan Gure Yinco Yaüna -hua cen yondoni Betiriikin -yeiki cen burua dena distiranta eta erran çacoten: "Ciek pobreak eta onak ciezte: cien auzoak abeassak eta gachtoak, Yincoaen yusticia eina içan dailla!”. Behaala lurra ikharatucen Yesus eta yondoni Betiri endurtu cien eta bi zaharrak eroï zien belauniko... Bihaamun goicean nahi içan çutenean yoan herrirat ce pasatu cen yakitea etçuten ikhusi batee etcheik, heeen lekhuian ciphu bat; oono eüngo eünean Barbazaneko herriaen eta Saint-Bertrand-de-Commingeco heriaren artean kausitcen dena. Lagundu behar dia beharrac.

\section{oOo}

\section{Tantugouen erran çaharra.}

Tantugou da guizon çahar bat oyhanetan gordetcen dena. Landak eta phenceak çaincen ditu handik ez dezaten yeusik ebats. Belharra eta oguiac eakiak dienean, nausiak denak sarthou artio Tantugouk ez du batee lo eiten; bethi iatçartua daü. Hua aguercen ikhusteco beldurrac atchikitcentu ohoïnak. Ona da sinhetsacie bainan basa da. Laborari hanitz gaüza zakien batec erraten ceen ardura bere semer Tantugou cela lurreco fruituen zainçaleik hobeena.

Uztaileco arratsalde batez artalde bat alhatcencen mendi baten gainean, artçaina lokartucen: iatçarcean ikhusi cien Tantugou ihesi yoaiten oyhaneco aldeat. Berdin eçaütuya içan cen ordoki pullit batean etçana harroca baten gainean.

Eungo eunean oono mendietaco herri çombaitetan haan beldur dia Mutico ttipi batek edo nechcato ttipi batek ez tienean bere amari obeitu nahi, hunec erraiten daco: Casu eisak! Tantugou deitcen diat eta behaala yien duk oyhanetik.

Instituteur: Dibar, né à Louhossoa, le 28 8bre 1861 Coll.: Mendilahaxou J, Mme Detchessarry, M Babaquy Luc 
Bacen lehenago Pyrénetaco herri batian guizon bat eta emazte bat zoinac baitzien arras zaharrac. Etzuten etche ttipi bat, baratze bat eta behi bat baizic. Bainan nahi becen pobriac izanic ere, sokoritcen ziusten beti berac baino pobriago zienac. Egun batez lurra estalia cen elhurrez, harria arrailtzeco horma haricen. Herrico yende guziac berotcen zauden, yatian eta edatian, aberatsac ziren eta urusac. Ilhunirian, bi piyayant, zoinac heldu baitziren urrunianic nahi ukan zuten guelditu leku hortan ceren eta hotz eta gose baitziren. Yo zuten borta batian guero beste batian eta bestetan oraino: nehorc etziusten sartzeat utzi nahi, ez eta ere deus yaterat eman. Casatiac orotaric seguituac chakur samurrez, bi estranyerek etzakiten norat yuan. Kausitu zienian herriaren basterrian, guizon eta emaste pobre horien etchearen aintzinian. Aitziarekin heyen arrangurac, senharra emantxen leyhuan heyen deitzeco eta haren emastia yuan cen fite bortaren idekitzerat. Galde eguin gabe nor ziren sarrarazi ziusten eta yarrarazi supasterrian; guero, eman tzayoten esnia eta gaztainac, zuten gucia. Bainan ordian, gure Yauna; -hua cen Yondoni Petirikin- chutitu cen, burua dena distirant, eta erran tzakoten: "Zuec pobreac eta onac zieste; zien hauzuac aberatsac eta gaichtoac dira. Yaincoaren yustizia eguin dadiela!". Berehala lurra ikaratu cen, Jesus eta Petiri galdu zien eta bi saharrac erori zien belhaunico... Biharamun goizian nahi ukan zutenian yuan herrirat yakiteco cer pasatu cen, etuten ikusi etche bihiric: heyen lekian, bacen ur handi bat, zoina kausitcen baita oraino egun Barbazaneco herriaren eta San Bertrand de Comingeco hiriaren artean.

Behar dire malhurusac lagundu.

\section{oOo}

Tantugou da sahar bat: zoina gordetcen baita oyhanetan. Zaintcen tu landac eta pentziac deusic ez dezaten ebats handic. Oguiac pikatuac ita belharrac segatuac cienian, oro nausiaz erretiratuac izan arte, Tantugouc ez tu loric eguiten; beti beillatcen tu hua aguer dadien beldurrac atchikarasten tu ohoinac. Ona da, sinhetz zazue, bainan salbaya. Hainitz gauza zakien laborari batec, erraten zioten usu bere semer Tantugou cela segurki lurreco fruituen zeintzale hoberena.

Ustaillaco eguerdi ondo bates, ardi tropa bat alha cen mendi baten gainean, artzaina lokartua cen: iatzartziarekin, ohartu cen Tantugou ihes yuan cela oyhanerat. Guisa berian ezagutua izan cen artsainez mendi sola polit batian, etsana arroka baten gainian.

Orainon egun, Pyrenetaco herri batzuetan, haurrac haren beldur dira. Mutico ttipi eta neskatoce ttipi batec obeditu nahi ez dienian bere amari, erraten daco: Guardimac hiri! Deitzen diat Tantugou ita berehala, yinen duc oihanetic.

Instituteur: Hiribarren, 23 ans, né à Bidart Coll.: Brana et Mendivil 
Lehenago bazien Bachenabarreco mendietaco heri batian guizon bat eta emazte bat biziki zaharrac zienac. Etzuten etche ttipi bat, baratze bat eta behi bat baicic. Bainan nahi bezan pobriac izanic ere bethi laguntza eguiten zuten berac baino beharaguer. Egun batez lurra elhurez kukutia zen eta arailltzeco horma bat ari. Herrico yende guziac berotzen zauden yaten eta edaten ari zielaric. Aberatxac eta urrusac ziren. Ilhundirian bi piaïant, hurruntic heldu zirenac guelditu nahi izan ziren lekhu hartan ceren eta hoztiac eta gosetiac beitziren. Yo zuten bortha batian, guero beste batian eta oraino beste zombaitetan. Nihorc etziuzten sartzera uzten ez eta deus yatera emaiten. Orotaric kasatiac, sakhur samur batzuez seguitiac, bi estranyerec etzakiten norat yoan, noiz ere heriaren baztérian khausitu beitziren guizon eta emazte probre hen etchiaren aitzinian. Hen plainiac entzunic, senhara emantzen leihuan oihu éguiteco eta emaztia yoan zen fitechko bortharen idekitzera. Galdeguin gabe ere nor ziren, sar erazi ziuzten eta yar erazi suphazterrian, guero zerbitzatu zacoten esnia eta zombait gaztena, zuten guzia. Bainan ordian, gure Yincoa, hura zen yodoni Bethiriekin - chutitu zen buria dirdirantic eta eran zacoten: "Ziec pobriac zizte bainan onac, zien auzoac aberatxac dira, bainan gachtuac. Jincoaren yusticia eguina izan dadiela! Orduian berian lurra ikharatu zen, yésus eta yodoni Bethiri galdu ziren eta bi zaharrac eman ziren belaüco. Bihamun goizian, noiz ere herrirat yoan nahi izan beitziren, yakitera zer pasatu zen, etzuten etche bihiric ikhusi: hen lekhuian bazen ur handi bat egun oraino khaüsitzen dena Barbazaneco herriaren eta SaintBertrand de Commingeco herriaren artian. Behar dia maleusac sokhoritu.

\section{oOo}

Tantugou oihanetan gordatzen den guizon bat da. Landac, pentciac zaintzen ditu deusic ebatx ez dezaten. Noiz ere oguiac phikatiac beitira eta belhara ebakia, nausiac denac sar artio Tantugouc ez du loïc eguiten, bethi zain dago. Aguer dadin beldurac atchikitcen ditu ohoinac. Pherestia da, sinhesten ahal nuzu, bainan bacha da. Hanitz gauça zakien laborari batec eraiten zin bere semer, Tantugou zela segurki lurreco fruitien zain zalerik hobena.

Uztailaco aratxalde batez, arthalde bat alhazen mendi batian eta artzaina loac hartu zen. Iatzartziarekin, ikhusi zin Tantugou ihex eguiten oihanerat. Ezagutia izan zen ere aberezain batzuez, erephea poillit batian, harroca batian etzana. En oraino, Bachenabarreco mendietaco herri batzuetan, haürrac haren beldur dira. Noiz ere mothico edo nescato ttipi batec ezbeitu nahi amari obeitu, eraiten dago: khasu eguic, oihu eguiten badagot Tantugouri, abian yinen duc oihanetic!

Instituteur: Ayphassorho, né à Gotein-Libarrenx le 16 8bre 58 Coll.: Harismendy J., Iriberry, Cadet 
Diila aspaldi, bazi-in Pyrenetaco herri batian guizon bat eta emazte bat arras zaharrac. Etche ttipi bat, baratze bat eta behi bat bezic ez zuten. Bainan launtzen zuzten berac beno pobrioocoac. Eun batez, lurra elhurrez cucutia zen eta horma handi bat zen. Herrico yende guziac berotzen zi-in yatia-akin eta edatiaakin. Aberas zi-in eta urus. Eun batez, bi piayant, urrundanic heldu zi-inac, nahi izan ziin guelditu lecu hartan, zeren eta hotz eta gosiac baitzi-in. Yo zuten bortha bat, guero bertze bat, eta guero bertze zombeit. Nihorc ez zitien sar arazi nahi eta yaterat eman. Orotaic casatiac, zacurrez hersatiac, bi piayant hec ez zakiten norat yuan. Orducotz herria-an beste bazterrian zi-in, guizon eta emazte pobre heen etchia-an aitzinian. Aitzia-akin heen arrangurac, senarrac leihotic deithu ziti-in eta emaztia bortha-an idokiterat yuan zen. Galdegin gabe nor zi in, sar arazi zituzten eta su bazterrian yarrarazi. Guero eman zazcoten esnia eta gaztenac: ez zuten bertzeic. Bainan ordian, Yincoac -hura zen Yende Petiriikin- yaiki zen, buria dirdiran eta erran zacoten: zi-ic pobriac zi-izte eta onac; zi-in auzoac abaatsac di-i eta gachtuac. Yincua-an yusticia ina izan dadi-ila. Ordian lurra icaratu zen. Jesus eta Yendee Petiri ezeztatu zin. Guizon eta emazte zahar hura belhauncatu. Biharamun goizian, nahi izan zutenian yuan herrirat yakiteco zer guerthatuzen, ez zuten etche bihiric icusi. He-en lekian, ur batzen. Ur hua bera da, oono ere Barbazan deitzen den herria-an eta Bertrand de Comminges deitzen den hiria-an artian. Behar di-i malurusac lagundu.

\section{oOo}

Tantugou da guizon zahar bat gordetzen dena oihanian. Zaintzen ditu landac eta pentziac deus ez dezaten ebax. Ogiac edo belharrac ebakiac di-inian, nausiac oro bildu artian, Tantugou ez da locartzen. Bethi guardia do-o. Ohoinac ha-an beldur di-i. Yakin zazu ona dela bainan basa. Gauza hanitz zaki-in laborari batec erraten zi-in maïz bere semer Tantugou zela segurki lurreco fruitien zainzaleric hoberena.

Uztaileco axalde batez, ardi tropa bat alha zen mendi batian; artzaina locartu zen: iatzartzia-akin, icusi zi-in Tantugou, oihanerat ihes eguiten. Ezagutu zuten ere artzain batzi-ic, zelhaï eder batian, etzana harroca baten gainian.

Oono ere, Pyrenetaco herri batzi-itan, haurrac ha-an beldur di-i. Muthico edo neskato tipi batec obeditu nahi ez di-inian amari, erraten daco amac: Guardia emac! Ekarrazten diat Tantugou! Behala yi-in duc oihanetic.

Instituteur: Berterêche, $\mathrm{J}^{\mathrm{n}} \mathrm{B}^{\mathrm{pte}}-31$ ans, né à Itxassou, B.P. Coll.: Etchecopar, 75 ans, Diron Etienne, 78ans. 


\section{Barbazaneco ichtoriua Bescoitzeco herrico lenguayan}

Lehen baziutzun Pyrenetaco herribatetan gizon eta emazte bat biziki zaharrac. Etzitzien etche ttipi bat, baratze bat eta behi bat bezic. Bainan hain pobre izanaatic laguntzen zitzien bethi berac bano pobriago zienac. Egun batez lurra elhurrez estalia zuzun eta horma ari harriac erdiratzeco phunduraano. Herrico yende guziac berotzen ari ziutzun yanez eta edanez; abats ziutzun eta urus. Ilhun bazterrin, urrundanic heldu zien bi piayant nahi izan ziutzun gelditu toki hartan, zeen hotz eta gose baitzien. Yo zizien bortha bat geo bertze bat eta bertze batzu oono: nehoc etzitzien utzi nahi ukhan sartzea ez deus eman yatea. Casatuyac orotaic, segituyac chakhur borthitz batzuyez, bi arrotzec etzakizien noat yuan khausitu zielaic herriaan bazterrin gizon eta emazte pobre heyen etcheco bortha aitzinian. Aitziakin heyen plainuyac senharra yali zuzun leihoa, heyerri oihu eitea eta emaztia yuan laster borthiaan idekitea. Galdein gabe nor zien, sarraazi zitzien eta yarraazi been suphasterrin: geo eman zacotzien esnia, zonbait gaztena, ziuzten guziac. Bainan orduyan Yinkua - hua zuzun Yondoni Petriikin- yeiki zuzun buruya arras distian eta erran zacozien: "Ziec pobre zizte eta on, zien auzuac abaats tutzu eta gaichto". Behaala lurra ikhaatu zuzun; Yesus eta yondoni Petri itzaldu ziutzun eta bi zaharrac erori belhaunco...

Bihaamun goizin, nahi izan zienin yuan herrirat yakiteco ze phasatuzen etzizien ikhusi etche bat ere: heyen lekhuyan bazuzun hur tulunbio handi bat. Oono egungo egunin Barbazaneco herriaan eta san-Bertran-Comminyekuaan artin khausitzen dena.

Maluusac behar die soleitu.

oOo

\section{Tantuguren ichtoriua}

Tantugu duzu oihanetan gordetzen den gizon zahar bat. Zaintzentizu landac eta phentzic nehoc ezdezaten deusic ebats. Ogiac phikatuyac dienin edo belharra segatuya, denac nausiac barnerat eman artin, Tantuguc ezdizu batee loic eiten, bethi iatzarriic doozu. Hua agertuco delaco beldurrac zaintzentitzu ohoinac. Onauzu, sinetsazii, bainan salbaya. Anitz gauza zakiin laborari batec erten zizun maïz bere semerri, Tantugu zela seurki lurreco fruituyen zaintzaleic hobeena.

Uztaileco atsalde batez ardi tropaat alhatzen zuzun mendi baten gainin, artzaina lokhartu zuzun. Iatzartzian ohartuzuzun Tantuguc ihes eiten ziela oihaneco aldeat. Ezautuya izan zuzun oono artzain batzuyez, haran poillit batetan etzana harroca baten gainin.

Egungo egunian oono Pyrenetaco zonbait herrietan, haurrac harren beldur diutzu. Muthico ttipi batec edo nechkatoco ttipi batec ezdienin obeitu nahi bere amai, hunec erten dacozu: Khasu hiri! Oihu eiten dacoyat Tantuguri eta ichtantin yienduc oihanetic!

Instituteur: Jauréguiberry, trente ans, né à Jaxu (B. Pyrénées) Coll.: Dourisboure, 65 ans. 


\section{Barbazaneco hichtorio chaharra}

Baciren demboran Pyrénétaco herri batean guiçon bat eta emazté bat biciki çaharrac. Ez çuten etche ttipi bat, baratcé bat eta behi bat baicic. Bainan nahi beçain pobriac içanic éré, laguntcen cituzten bethi berac baino pobréaguac. Egun batez, lurra estalia cen elhurrez eta horma ari cen carrascaz. Herri hartaco yende gucia berotcen cen yanez eta edanez; aberats ciren eta ourus. Ilhun cerratcean, bi pasayant ourroundanic heldou cirenac guelditu nahi içan ciren leku hartan, ceren gosé eta hotz baitciren; yo çuten bortha bat, guero bertce bat eta bertce çombeit oraino: nehorc etcituen utci nahi içan sartcerat, ez eta deusic eman nahi içan yatera. Orotaric casatuac, seguituac chakhur gaichtoès, bi arrotcec etcakiten norat yoan, noiz eta éré causitu baitciren herri haren basterrean, pobreac çirelaco guiçon eta emazte heien etchearen aitcinean. Heien plainuac ençutearekin, senharra eman cen leihoan, heien galdeguiteco eta haren emaztea yoantcen fité bortharen idokiterat. Galdeguin gabe heia nortciren sarrarasi cituzten eta yarrarasi béré suphazterréan, guéro serbitçatu ceten esnea eta çombeit gazténac çuten gucietaric. Bainan orduan Yésus Chrichto hura cen yondoni Bettirirekin, chutitu cen, buru gucia dirdiran eta erran ceten: Ciec pobreac ciezté eta onac, cien auçoac aberatsac dira eta gaichtoac Yaincoaren yusticia eguin bedi. Berehala lurra ikharatou da, Yésus eta Bettiri galtcen dira eta bi chaharrac emaiten dira belhauninco. Biharamun goicean, noiz eta éré yoan nahi içan baitciren herrirat, yakiteco ce phasatu cen eztçuten ikusi etché bihi bat heien plaçan nasa bat cen, Barbazaneco herriaren eta San-Bertrand Comminjaco hiriaren artean oraino egungo egunian causitcen dena. Behar dira malurusac lagundu.

\section{Tantugouren historio chaharra}

Tantugou guiçon çahar bat da oihanetan gordatcen dena. Beiratcen ditu landac eta phentceac deus ere ebats ez deçaten. Oguiac phicatuac direnean edo belharrac ebaquiac, guçiac nausiaz bilduac içan artean Tantugouc ez du loric batere eguiten; bethi guardian dago. Haren aguer dadin beldurrac beiratcen ditu ohoinac. Ona da sinhets atçué, bainan basa da. Laborari batec çoinac baiçakien gauça frango cion ardura bere semer Tantugou cela segurki lurreco fruituen guardiariric hoberenac.

Ouztaileco hilabethean eguerdi ondo batez, ardi tropa bat alha cen oihan batean; artçaina lokhartu cen iratçartcean ohartu cen Tantugouri coinac ihes baitçohan oihaneraco aldérat. Bardin eçagutua içan cen, behi ceinez aphalgune poïllit batean etçana harroca baten gainean.

Oraino egun Pyrénéco herrichca batçuetan haurrac haren beldur dira. Muthico edo nechcato ttipi batec obeditu nahi ez duenean bere amari erraiten dio "Guardia emac deitcen diat Tantugou eta berehala yinen duc oihanetic. 
Baciren lehenago Pyrenetaco heri batian guiçon bat eta emasteki bat hanitz çaharac. Ex çuten etche tiki bat, baratce bat eta behi bat baicic. Bainan hain beharac ciren aren, laguncen citusten bethi beharagoac. Egun batex lura estalia cen elhurrez eta horma cen arailagaria. Herico yende guciac berotcen çagocin, yaten eta edaten çutelaric; aberats eta urus ciren. Ilhun nabarian, bi piayant, urundic heldu cirenak, nahi içan çuten guelditu toki hartan, ceren hotz eta gose baitciren; yo çuten athe bat, guero berce bat eta berce batçu oraino, nihorc ez cituen nahi içan sarcerat utci, ez deusic yaterat eman. Gucietaric cassatuac, chacur borthitcez seguituac bi piayant arotcec ez çakiten norat gan, noiz eta ere causitu baitciren heriaren basterian, guiçon eta emaste behar horien etchiaren aitcinian. Hekien dolamenac aditciarekin, senhara athera cen leyhora oyhu eguiteco eta emastea gan cen laster athiaren idekitcera. Galdetu gabe nor ciren, sararaci citusten eta yararaci suphasterian; guero eman cioten esnia eta cembeit gastain, cuten gucia. Bainan orduan Gure Salbatçailia -hura cen Yondoni Petrirekin, chutitu cen buruac disdiratcen cioelaric eta eran cioten: Çuec beharac çarete eta onac; çuen auçoac aberatxac dire eta gaistoac. Yaincoaren yusticia eguin dadiela. Berehala lura ikharatu cen, Yesus eta Yondoni Petri sunsitu ciren eta bi çaharac erori ciren belaunico. Biharamun goician, nahi içan çutenian gan herirat cer guerthatu cen yakiteco ez çuten ikusi etcheric batere. Hekien tokian cen putçu bat, çoina causitcen baita egun ere Barbazan heriaren eta Saint-Bertrand-deComminges heriaren artian.

Behar dire beharac lagundu.

oOo

Tantugou da çahar bat gordetcen dena oyhanian. Çaintcen ditu landac eta sorhuac, deus ex deçaten ebatz. Oguiac eta belharac phicatuac direnian, guciac nausiac sarthu arte Tantugou ez dago lo; çain dago bethi. Hura aguercen ikhusteco beldurac ohoinac atchikitcen ditu, ona da, sinhetz çaçu, bainan salbaya da. Laborari batec çoinac baitcakien hanitz gauça, eraten cion maix bere semeari Tantugou cela segurki lureco fruituen çainçale hoberena.

Ustailaco aratxalde batez ardi arthalde bat alhatcen cen mendi batean; arçaina locartu cen; atçarciarekin ikusi çuen Tantugou ihesi çoala oyhanerat. Eçagutua içan cen arçainez haran puillit batean etçana haroca baten gainean.

Oraico egunian ere, Pyreneco cembeit heritan haurec haren beldura badute. Noiz eta ere mutico tiki batec edo nechca tiki batec ez baitu nahi obeditu bere amari, eraten dio Gardia emoc! Oyhu eguiten du Tantugou eta berehala ethorico da oyhanetic. Pyrénées).

Instituteur: Lescarboura, âgé de quarante-un ans, né à Labastide-Villefranche (Basses-

Coll.: M. Berterrèche Laurent, instituteur en retraite Observations (inutiles peut-être pour le travail demandé). 
68

En orthographe basque le z répond au son du ce ou s. On a généralement renoncé au ç; mais le basque a un autre s nommé rude, que le français ne connaït pas. D’après ces principes, Gasteluzar est écrit d'une manière fautive attendu que les Basques prononçons Gaztelu et non Gastelu. Cette manière d'écrire pour être justifiée au point de vue étymologique Gasteluzar (vieux fort) formait le premier chaînon d'une ligne de défense formée par les Basques, contre les attaques des Francs et dont on a trouvé les traces jusqu'à St-Jean-Pied-de-Port (Basses-Pénés), en arrière d'obstacles naturels. Or, le mot Gasteluzar, vient évidemment du latin castellum. En un mot si Gastelu est mal orthographié au point de vue de la langue basque, il est régulier au point de vue étymologique (Duvoisin, capitaine des douanes en retraite). 
Baciren bertce orduz (demboraz) Pyrenetako herri batean guiçon eta emazte bat arras çahartuak cirenak. Ez çuten etche tiki bat beicik, baratce eta behi bat. Egun batez lurra elhurrez estalia cen, horma hari cen harriak berak arrailtceco menian. Herriko yende guciak yaten, edaten eta berotcen hari ciren; urus eta aberats ciren. Ilhun nabarrean bi bidairante urhundik heldu cirenak, guelditu ciren herri hartan. Ecen gose eta egarri ciren. Athe yok ibilli ciren etchez etche. Nehork ez cituen sartceat utci nahi, nehork ez cioten yaterat eman. Egoitça gucietaik urhunduak (igorriak), çakur assaldatuez berez seguituak, bi kampotiarrak (bidairantak) ez çakiten norat gan, noiz eta ere kauusitu baitciren herriaren mugan, aiphatu ditugun guiçon eta emazte erromesen athe aintcignean. Heien errenkurak aditcearekin senharra pharatu cen leihoa eta deithu cituen eta haren emaztea gan tcen laster athearen idekitceat. Galdetu gabe nor ciren, sar-araci cituen eta yar araci. Gero eskaigni (eman) cioteiten esnea, gaztagnak eta cituzten guciak. Bagnan orduan Yesuchristo gure yauna (hura cen yondoni Petriekin) alchatu cen burua dichtiraduraz inguratua eta erran cioten: "Çuek erromesak çaizte baignan bihotz onekoak (onak). Çuen hauçoak aberatsak dire baignan bihotz gohorrekoak (tcarrak) Yainkoaren yusticia eguin dadiela! (cigorradak yauts didala)". Bereala lurra ikaratu cen, Yesuchristo eta Yondoni Petri gorde ciren eta bi senhar emazte çaharrak belhauniko pharatu ciren... Biharamun goicean herrirat gan nahi içan çutenean, Yakiteco cer gerthatu cen, etçuten etche bakar bat ikusi: Heien lekuan bacen ur égoitça çabal bat; oraigno egungo egunean ikus daitekena, Barbazango eta Saint-Bertrand-Commingesgo herrien artean. Behardire lagundu behardunak.

\section{oOo}

Tantugou da guiçon çahar bat oihanetan gordetcen dena. Caintcen ditu landak (ordokiak) eta sorhuak deusik ez deçaten ebats. Noiz eta ere oguiak eta belharrak pikatuak baidire nausiak hek sarthu arte, Tantugouk ez du lorik eguiten. Bethi atçarria dago. Hura ikusteco beldurrak urhuntcen ditu ohoinak. Bihotz onekoa da (ona) baignan salbaïya (bicitcen ikasi gabea). Lur languille batek, gauça hanitz eçagutcen çuenak erraten çuen bere semeri, ez cela nihor Tantugou becin ontasun çaintçaille segurik.

Uztailleko arrats alde batez, ardizko arthalde bat iragaiten cen mendi gain batean. Artçaigna loharkatu cen: ihatçartu cenean, ikusi cuen Tantugou oihanari buruz ihesi çohala. Guise berean eçagutua içan tcen celaietaco artcain batcuez, etçana çagolarik harroka baten gainean.

Oraino egungo egunean, Pyrenetako cembeit herritan haurrak icitcen ditu. Noiz eta ere mutiko edo nechka tiki batek ez baitu bere amaren errana egin nahi, amak errate dio: "Kontu emak! Deithuko diat Tantugou, eta berehala ethorriko duk oihanetik. 
Bacien lehenoo Pyrenetaco herri batian guizon bat eta emastequi bat arras caharrac cienac. Ezuten etche ttiqui bat berceic, baatce bat eta behi bat. Bagnon, hain pobre izanique, socorricen ciusten hec bagno pobrio cienac. Eun batez, lurra elurrez estalia cen, eta orma eiten çuen arria arraillaceco. Herrico jende guciac berocen ai cien, jatian eta edatian, aberats eta urus cien. Ilhun aarrian, bi piaient urrundic heldu cienac, leku artan guelditu nahi izan zuten, ceren hotz eta gose baizuten; yo zuten ate bati, guero berce bati eta oogno berce bazueri: nioc etzituen utzi nai sarceat, ez deusic eman nai yateat. Casatuac gucietaic uiatuac sakur amurratuz, bi estranyerec etzaquiten noat gan, causitu cienian herriaan akanzan, guizon eta emazte pobre cien eien etchian aitzinian. Aitziaquin equien errenkurac, senarra eman cen leiuan ekien deitceco, eta arren andria gan cen laster atian idequitcea. Galdatu gabe eier nor cien; sarreci zusten eta yarraci beren supasterrian; guero cerbizatu ciozten esnetic eta castagna bazuec, zuten gucia. Bagnon orduan, Gure Jauna -ura cen Jondoni Petriquin - alchatu cen, burua dena distiranta, et erran cioten: "Zuec, pobriac zaezte eta onac; zuen auzuac abatsac die eta gaistuac. Jincuan justicia ein daiela!". Orduan berian lurra icaratu cen, Jesus et Jondoni Petiri esestatu cien eta bi zaharrac erori cien belhaunico... Bihamun goician, herriat juanahi izatu cienian, jaquiteco zer pasatu cen, etzuten icusi etche bihic: heien plazan bacen ur bildu bat; zoina causicen baita oagno Barbazaneco herriaen eta Saint-Bertrand-de-Commingeco hiriaen tartian. Behar die laundu malusac.

\section{oOo}

Tantugou zahar bat da oihanetan gordecen dena. Zaincen tu landac eta sorhuac deusic ez tezaten an eats. Oguiac picatuac dienian edo belarra picatua, dena nausia eraman izan arte, Tantugouc ez tu bate loic eiten; beillan do beti. Beldurrac ua icustiaz aguercen achiquicen tu ohoinac. Ona da, sinhes zazue, bagnon basa da. Laborari batec zaquisquinac asco gauza erraten zuen maïs bere semeri Tantugou seurqui lurreco fruituen zainzaleic obeena zela.

Uztailleco ilateco aatsalde batian, ardi tropat alha zen mendi batian; arzaina locartu cen: iarzaziaquin, aperceguitu zuen Tantugou ihes ai zoala oihaneco parteat. Ezautua izatu zen igual arzaines errepira pullit batian etzana arroca baten gainian.

Oagnic egun, Pyrenetaco zombeit erritan, haurrac arren beldur die. Muttico ttiqui batec edo nechca ttiqui batec eztuenian obeitu nai bere amari, erraten dio: Guardi emac iri! Deitzen diat Tantugou eta, istantian etorricouc oihanetic. 


\section{Barbazango khondera}

Bertze orduz baziren Pyrénées-tako herichka batean senaremazte batzu zaharkituak. Etche ttiki bat, baratze chume bat aldean eta behi bat ziren heikien ontasun guzia. Nahiz aurkhitzen ziren beharrak laguntza ematen zioten berak baiño beharrago zireneri. Egun batez lurra estalia zen elhurrez eta hormak, errana den bezala, arailatzen zituen harriak. Herichka huntako yende guziak berotzen zauden janez eta edanez ahal zuten guzia; aberats ziren eta zorion. Gaü haren sarreran hurrundik heldu ziren bi bïaiantek nahi izan zuten lekhu hartan trikatu hotz eta gose zutelacotz; hemen jotzen dute athe bat, haratago bertze bat eta bertze haiñitz oraiño: nehork ez zioten ideki ez eta ere deusik yaterat eman. Alde guzietarik ohilduak, zakhur tzar batzuek jaraikitzen zitzaizkotela, bi bïaiant haukiek ez zakiten norat gan, noiz eta ere khausitu baitziren hirietako hegian, aurkitu ziren aiphatu senaremazte hekien etchiaren aitziñean. Berehala senarrak aditu zutieneko haukien hauen-miñak agertu zen leihorat haukien bere etcherat deitzeko eta emaztea ordu berean gan zen athearen idekitzerat. Bathere galdetu ere gabe nor ziren sarharazi zitusten barnerat eta yar harazi su pasterean; gero eman ziotzaten esne beroa eta zombeit gaztaña, etchian ordu hartan zituzten yanarri bakharrak. Orduan Gure Yauna -ezik hura zen Jondoni Piarresekin,- chutitu zen burua iguratua dichtiradura miragarri batez eta erraten diote: "Zuek beharrak zaiste, baiñan yende onak; aldiz zuen hauzoak aberats eta tzarrak. Yainkoaren zuzentasunak egin beza bere bidea."Ordu berean lur ikharra yauzi zen eta Yesus eta Piarres suntsitu ziren; bi zaharrak aldiz belhauniko ahuspez lurrerat erori ziren... Biharamun goizean, noiz eta ere abiatu baitziren herrirat yakitekotzat zer iragan zen, ez zuten han ikhusi gehiago etcherik eta haukien orde aurkhitzen zen intzurra handi bat, gaurko egunean aurkhitzen dena oraiño Barbazan-go herichkaren eta Saint-Bertrand-de-Cominges-co hiriaren artean.

Bethi lagundu behar dire zorigaitzezkoak.

$$
\text { oOo }
$$

Tantugou da gizon zahar bat oihanetan gordetzen dena. Begia atchikitzen diote landa eta sorhueri handik ez dezan nehork deusere ebats. Ogiak eta belharrak ebakiak direnean, yabeak hek denak barnean sar detzan arte, Tantugouk ez du lorik batere egiten. Bethi atzarria dago. Haren nonbeitik atheratzen ikhusteko beldurrak berak hurun atchikitzen ditu ohoiñak. Siñets azue hau, arras ona da berenaz baiñan basatiara da. Bazteretchar zahar batek, zoina baitzen yakinsunik handienekoa, maiz aski eraten zioten bere semeeri, Tantugou zela egiazki lureko fruituen zaiñtzalerik oberena.

Uztailiako aratsalde eder batez artzaiñ bat lo dorphe batek hartu zuen bere arthaldea halatua zaukan denboran mendi baten gaiñean. Betbetan atzartzen da eta horra non ikhusten duen Tantugou ihesi dohala oihaneko alderat. Alaber denbora pertsuan ezagutua izan zen bertze artaiñ batzuez zelai puillit batean aroka baten (aldean) gaiñean ertankara etziña. 
Gaurko egunean ere Pyrénées-tako hirichka zombeitetan haurak haren beldur dire. Mutiko edo nechka ttiki batek ez duenean bere ama obeditu nahi, ez duenean hunek errana egin nahi eraten dio: "yakin zak haü ez bauk nik errana berehala egiten, Tantugouren deitzerat nihoak eta laster ethorriko duk hire billa oihanetik".

Instituteur: Ahetz-Etcheber, né à Ste Engrace le 9 Mars 1851. Coll.: Haramboure, Bessan, Lameur 


\section{Barbazanco erran zaharra}

Bacen bertce orduz Pyrenetaco herri batean gizon bat eta emaste bat hainitz zahartuac. Ez zuten etche ttiqui bat, baratce bat eta behi bat baicen. Bainan nahi baicen probiac izanic ere socorritcen cituzten bethi hec baino probiago cirenac. Egun batez, lurra estalia zen elhurrez eta horma eguiten zuen harriac arraildu arte. Herrico yende guciac berotcen hari ciren, yatian eta edatian, aberatz ciren eta urus. Ilhuntcian, bi piyayant heldu cirenac urrundic, nahi izan zuten guelditu toqui hortan, ceren hotz eta gose baitciren; yo zuten athe batian, guero bertce batian, eta oraino bertce batzuetan. Nihorc ezcituzten sartcerat utci nahi izatu. Casatuac orataric, chakuac gaichtoez perseguituac, ez zaquiten bi estranyerec norat gan, causitu cirenean herriaren bertce basterrean guizon eta emaste probe hoiquien etchearen aitcinean. Aditcen hoiquien plaignuac, senharra emancen leihuan deitceco eta haren emastea ganzen laster athiaren idequitcerat. Galdetu gabe norciren, sararaci cituzten eta yaraci beren suphasterrian; guero cerbitzatu ciozcaten esnia eta gaiztaignac, cituzten guciac. Bainan Gure Yauna, ceren huracen Yondoni Petrirequin, chutitcen da, burua distirantic eta erraten diote: "Zuec pobriac zareste eta onac, çuen haüzoac aberatsac dire eta gaichtoac. Yaincoaren yusticia eguin dadila. Cuchian lurra ikaratcen da, Yesus eta Yondoni Petri desguisatcen dire eta bi zaharrac erortcen dire beelhaunico... Biharamunean, nahi izan zutenean gan herrirat yakiteco cer pasatu cen, es zuten ikusi etche bihibat: hequien plazan bacen lacua bat, egungo egunian hatchematen dena oraino, Barbazaneco herriaren eta San-Bertrand-Commingeco hiriaren artean. Malurusac, behar dire lagundu.

\section{Tantuguco erran zaharra}

Tantugu zen guizon sahar bat, oyhanetan gordetcen cena. Zaintcen cituen landac eta sorhuac, deusic ebatz ez cezaten. Oguiac edo belharrac phicatuac direnean, handic eta nagusiac denac alchatu arte, Tantugic ez dou loric eguiten, bethi atzarria dago. Tantiguren ikustearen beldurra atchiquitcen du ohoinac. Ona da, zinets zazue, bainan salbaïya. Laborari hainitz gauza zakien batec, erraten zuen maïs bere zemeri Tantugu cela segurki lurreco fruituen gardariric hoberena.

Uztailleko hilabetean, bascal ondo batez, ardi tropa bat mendiaren gainean alha cen; artzaina lokarcen da: artzarcean, ohartcen da Tantugu ihesi zohala oyhaneco alderat. Izancen higual ezagutua hazienda zain zales celhai puillit batean etzana harroca baten gainean.

Oraino egungo egunean zombeit herri Pirenetacotan haurrac beldur dire harentzat muthico ttiqui batec edo nechca ttiqui batec nahi ez dunean obeditu bere amari, harc erraten dio: Contu emac hiri! Oyhu eguiten cioyat Tantuguri, ichtant berean, ethorrico duc oyhanetic.

Instituteur: Payzan, 35 ans né à Livron 
Bacénn léhénago Pirénéétaco herri batiann guisson bat éta émastéqui bat çoïnac baïcirénac arras saharrac. Ez çoutenn etché ttiqui bat, baratcé bat, éta béhi bat baïcic. Baïnann cémbat nahi pobré içann citénn, lagountcénn çoutènn bèti hec baïnonn pobréago cirénac. Egoun batéz lurra elhourrèz est'aliacéna, éta horma haï çouenn harriac arraillatcéco guiçann. Hérrico géndi gouçiac bérotcénn hari cirénan, jaténn éta édatenn çoutélaric; abératz éta hourous cirènn. Hiloun habarriänn, bi piyayantec hourroundic heldou cirénec nahi içann çouténn guelditou lékhou hartann goce éta hotz çoutélacotz; tjo çutenn ate batiann, guéro bertcé batiann, éta oraïno bertcétann: nihorc ez citouenn sartcérat houtsi, ez éta héré tjatérat hémann. Egorriac toqui gouciétaric séguitouac sakhour fouriosés, bi estranyérrec ez çaquiténn norat jouann, noïz éta héré kaüsitou baïcirenn herriarénn bourouann pobre cire guiçonn éta émastéqui éhiénn etchéarenn aïcinéann. Aditcéaréquinn équienn plagnouac, cennharra émann cénn leïhouann éhienn déitcéco éta harenn anndréa juàtcénn laster atéa idéquitcérat. Galdétou gabé nor cirénn, çar haraci citousténn éta jar haraci çoucalde basterriànn; guéro cerbitçatou cioténn esnéa éta çémbéït gastagna çoutén goucia. Baïnann ordouann, gouré jaüna -hura cenn Tjondoni Petrirequin, alchatou cénn, bouroua déna distiranta éta erraténn dioté: Çuëc pobréäc çaïste éta onac; çouénn haöuçoac abératçac dire éta gaïstouac. Tjinncoärénn tjousticia éguina içann dadiéla! Béréala lourra ikaratcénn da, Tjéçous éta Tjonndoni Pétri çouncitou cirénn éta bi çaharrac éroriçienn belhaoünico... Biharamoun goïcéänn, nahi içann souténiann herrirat tjouänn, tjaquitéco cér passatou cénn, ézçauténn ikhouci etchéric batéré: ekiénn toquiann bacén our guéldi bat, çoïna kaöucitcénn baita gaöur oraïno Barbasango herriaren éta sànn Begnade Commingéco hiriärénn artéänn. Lagoundou behar dire erroméçac.

\section{oOo}

Tantugou da çahar bat gordetcénn déna égourrétànn. Saïntcen ditou landac éta sorhouäc ez déçatenn déouçic ébatz. Oguiac picatouäc édo belharra segatoua, naöuçiac denac sartou artio Tantugou ez da loharcatcen; béti erné dago, ogognac idoquiäc dire houra aguertcéco béldourrez. Ona da sinets çaçouë baïnann salbaya da. Gaoüça frango saquienn laborari batèc erratènn çouënn maïs béré sémééri Tantugou céla ségourqui lourréco frouïtouënn sainsailléric hobéréna bascal ondo batez, oustailéco ilhabétéänn ardi tropa bat tjaténn hari cénn méndi baténn gaïnéann; artçaïna loharquatou cénn: iharçarcéaréquinn, ikhousténn dou Tantugou escapatcen cela égourrénn aldérat. Orobat éçagoutoua içann cénn artçainez chabaldégui pollit batéänn etcinndouä arroca baténn gaïnéann.

Gaoür oraino, Pirénétaco çombaiit hérritánn haöurrèc harénn beldourra doute mutil ttiqui batèc édo netchéa ttiqui batec obéditou nahi ez douéniánn béré amari, hounec erraténn dio gardia hemac! Déitcénn diat Tantugou éta ichtàntiann etorrico duc égourrétic.

Instituteur: Choubac, 29 ans, né à Montory (B. P.) Coll.: M. Ansoborlo, Maire 


\section{Barbanzako erana}

Belhalaco egunetan, Pirénetaco herrichka batean, baziren senhar-emaztekia, arras zahartuac. Hekien izate guzia zen etche chumetto bat bere baratzearekin, eta behi bat. Bainan zembat ere beharrak baitziren, laguntza ematen zioten berak baino beharrago zireneri.

Halako egun batez, lurra elhurrez estalia zen, eta hari zuen horma bat harriak arailatzen zituena. Hango yendea berotzen zagoen, ongi janez eta edanez, hekiek aberats eta zoriondun baitziren. Ilhun nabarrean, bi arrotz bideant, hurrun danic zathortzinak, nahi izan zuten toki hartan baratu zeren gosiak eta hotzak baitzauden. Yo zuten athe batean, gero bertze batean, eta bertze batzuetan oraino. Nihork ez zituen sartzera utzi nahikatu, ez eta ere fitsic yaterat eman. Alde guzietaric ohilduac, zakhur gaistoak ondotik, bi arrotzak zabiltzan ez jakinez norat gan. Hola hola heldu izan ziren heriaren burura, aiphatu senhar-emazte beharren athera. Kampoko kuintak aditzearekin, gizona ilkhi zen leihora eta emazteak laster-egin zuen athearen idikitzerat. Galdetu gabe nor ziren sararazi zituzten eta supazterrean yarrarazi; eta gero eman ziozkaten esne eta zembait gastaina, zituzten guziak. Bainan orduan, Jesus gure Jauna, ezik hura bera zen jondoni Petrirekin, chutitu zen, burua distirant eta erran zioten: "Zuek, zaizte beharrak, bainan barnez onak; zuen hauzuak aberats dire, tzarrak aldiz. Jainkoaren zuzentasunak egin dezala bere bidea!". Ordu berean lur-ikarra jautsi zen: Jesus yauna eta Yondoni Petri galdu ziren bistatik eta bi zaharrak erortzen dire belhauniko.

Biharamun goizean, herrirat gan nahi izan zutenean, yakiteko zer gerthatu othe zen, ez zuten ikusi etche bihirik eta etcheen orde zen laco bat edo aintzira bat, hura baita egungo egunean oraino ikusten dena Barbanzaco herriaren eta San Bertran Comingoaren artean.

Behar dire beharrak lagundu.

\section{oOo}

Tantügou da gizon zahar bat, oihanetan gordetzen dena. Kontutzen ditu landak eta sorhuak ez dezatentzat handik deusik ebats. Ogiak eta belharrak phikatuak direnean, yabeak hek guziak barnean sarthuak ikus bizkitart, Tantügu ez da lo arkhatzen; bethi atzarria dago. Hura nonbaitik atheratuko delako beldurrak ohoinak hurrun atchikitzen ditu. Ona dela sinhets zazue bainan salbaya (basa) ere bai. Asko gauza zakien lur languille batek maiz eraiten zioten bere semeri, Tantügu zela egiazki lurreco fruituen zaintzalerik hoberena.

Uztailaco aratsalde bat zen; arthalde bat bazkatzen zagoen mendi baten gainean, artzaina lokartu zen. Atzarri eta ondoan, ikusi zuen Tantügu oihaneko alderat ihesi zuala. Halaber denbora bertsuan, bertze artzain batzuek ezagutu zuten, zelhai eder batean, haroka 
baten gainean etzana zaguela.

Gaurko egunean ere, Pyrenetaco herri batzuetan, haurrak izitzen ditu. Mutiko edo neska ttiki batek ez dioenian bere amari obeditu nahi, amak eraten dio: "Emak kontu! Oihu egiten diat Tantüguri, eta kuchian oihanetik ethorriko duk!"

Instituteur: Ahetz-Etcheber, Bernard, 38 ans, né à Licq-Atherey Coll.: Deluc, $\mathrm{J}^{\mathrm{n}}, \mathrm{B}^{\mathrm{te}}$. 
Bertce orduz, Pyrénetaco herri batian, baciren guiçon bat eta emaztequi bat arras çaharrac. Ez çuten etche chume bat, baratce bat eta behi bat baïcic. Bainan hainitz ciren arren pobre, laguntcen cituzten bethi berac baino pobriagoac. Egun batez, lurra estalia cen elhurrez, eta horma hari çuen harriac arrailtatceco heineraino. Herrico yende guciac berotcen hari ciren yaten eta edaten çutelaric: aberatx eta uroz ciren. Gaubaren hastian, bi pyayetaco presuna, urrundic heldu cirenec nahi içan çuten guelditu toqui huntan, ceren hotçac eta gosiac baitciren; yo çuten athe bat, guero bertce bat eta oraino bertce cembeit: nehorc ez cituen utci nahi sartcerat, ez deus ere eman nahi yateco. Igorriac gucietaric, huyatuac chacur gaiztoez, bi campotar horiec ez çaquiten norat guan; caustu cirenian herriaren buruan guiçon eta emazte heyen etchiaren aitcinian, ceinec aditcen baicituzten hequien plañuac, senarra athera cen leyorat hequien deitceco eta emaztea guan cen berehala athiaren idequitcerat eta galdetu gabe nor ciren, sar araci cituzten barneat eta yar araci sukaldian; guero eman cioten esnia eta cembeit gaztaina, horiec ciren cituzten guciac. Bainan orduan J.C gure Yauna (hura cen Yondoni Petrirequin) alchatu cen, burua arguitua, eta erran cioten: çuec pobriac eta onac çaizte; çuen hauçoac aberatxac eta gaiztoac dire, Yaincoaren justicia içan dadiela eguina. Berehala lurra ikharratu cen: Yesus eta Yondoni Petri esquisatu ciren eta bi çaharrac eman ciren belhaunico. Biharamun goician noiz ere guan nahi içan baitçuten herrirat yaquiteco cer berri cen, ez çuten ikusi etcheric batere; heyen lekuan cen ur lacubat, ceina causitcen baida oraino egun Barbazango eta san-Bertrand-Comminges coaren artian.

Bethi behar dire lagundu pobriac.

\section{oOo}

Tantugou da çahar bat gordetcen dena oyhanetan. Çaintcen ditu landa eta sorhoac deusere handic ez ebasteco; eta oguiac picatuac direnian eta belharrac segatuac, guciac nausiac barnerat bildu arteraino, Tantugou ez da batere lokartcen; bethi erne dago. Haren aguercea ikusteco beldurtasunaz, ohoinac ez dire atheratcen. Ona da, sinhetx çaçu, bainan salbaya da. Laborary batec, çaquiena hainitz gauça, erraten çuen bere semieri, Tantugou cela segurki lurreco fruituen çainçailleric hoberena.

Uztailleco hillabetian, eguberdi onduan, ardi oste bat pasatcen cen mendi baten gainian; arçaina lokartu cen eta iratçarry cenian ohartu Tantugou ihesi çohala oyhaneco aldeat. Eçagutua içan cen orobat arçainez, haran eder batian etzana çagola harroca baten gainean.

Egun oraino, Pyrenetaco cembeit herritan, haurac haren beldur dire. Noiz eta ere mutico chume batec edo nesca chume batec ez baitu obeditu nahi bere amari, hunec erraten dio: Contu emac, deitcen diat Tantugou eta berehala ethorrico da oyhanetic.

Instituteur: Espelette, 26 ans, né à Haux (canton de Tardets) Coll.: M.M Haira et Labarière 
Bacien lehenago Pyrenetaco herri batian, guizon bat eta emastequi bat arras zaharrac cienac. Ezuten éché ttipi bat berçeic baatce bat eta béhi bat. Bagnan hain pobre izaniqueré socorricen çitusten heyec bagno pobre cienac. Egun batez lurra élurrez estalia cen, eta orma éguitencuen arria arraitçeko. Hérrico yendé guciac bérocen ay cien, yaten et ératen, aberatz eta urus çien. Hilhun arrian bi piayazaillé urrundi heldu cienac leku artan guelditu nahi izatuzuten, çein, hotz eta gosé baizuten, yo zuten até batian guero bercian, ba eta berçe bazuetan, niok ézituzten nahi zarcen uzi, eta ez deusic yaten emannay. Egoriac dénétac eta sangatuac sakur amurratuez, bi estrandeye oriec ezakiten noat gan. Arribatun siènian erriaren akabanzat, guizon eta emastéqui pobre orien éché hanzinian, ayhuen playgnoac ayturic, senarra emanzen leiyuan, aìyeri huyu éguiteco, eta andria gançen beiyala atia irekiseat, galdetu gabé nor cien, yarraci situsten ben supasterria, guero emansioten eznia eta gastan basuec, situsten guciak. Bagnon orduan bérian Gure Yauna "ura bacen Yondoni-Petriquin". Alsatun çuen burua dena distirantirik eta eran çioten. Zuec pobriac eta onac sasté, eta zuen ayzuac abérazac eta gastuak diré, Yincoac yusticia éguin daiyela. Démbora bérian lurra hicaratu cen, Yesus eta YondoniPetri gan cien eta bi zaharrac érori belhaunico. Bihamun gocian izatu nay suténia gan errirat zer pasatuzen, ezuten ikisi éché bat, ben tokietan, hein plaçan bacen ur gueldi bat zoinac orayere Barbazaneco herriaren eta Saint-Bertrand-de-Commingeco hiriaren tartia bayta.

Beardiré lagundu malhuruzac.

oOo

Tantugou da guizon zahar bat oyenetan gordecen dena, zaincen tu landac eta sorruac deusic es dezeten ébazt. Oguia picatua dénian edo belharrac picatuac nauziac isanarté denac éremanac Tantugou es tu loic iten, béti beillazen dago. Beldurez ura aguerzia achikasten tu ogognac. Onada sinhes sasuez bagnon salbayada. Laborari batec bay bayzakien gausa franko erraten çuen berri semieri, Tantugou zela lurreko fruituen gardazalé onena. Uztailleco illeteco asalde batez, hardi tropa bat pazatucen mendi bateat. Arzaina lohacartu cen iyazar ciarekin ikusi suen Tantugou iguesi zuala oyanen alderat. Ezautua izatun cen arzaines errepila pullit patian etzana arroca baten gaynia. Ora éré Pyrenetaco zombeit erritan haurrac arren beldu diré Mutil ttiqui batec eta nechca ttiqui batec estuenia nay obéditu bére ama errantendio contu emac iri deitzen dia Tantugou, eta istantian étorrikoc oyanetu.

Instituteur: Cazebonne, 26 ans, né à Espiute Coll.: Claverie 
Gure tokietako herri batean baziren denboraz guizon eta emazteki bat arraz zaharrak zirenak. Ez zuten etche ttiki bat, baratze bat eta behi bat baizik, herri hekien izaite guzia. Bainan, nahiz behar ciren, berak baino beharrago cirenak sokorritzen zituzten bethi. Egun batez, lurra estalia zen elhurrez eta horma bat hari zuen harriak berak zartarazterainokoa. Herri hartan oro beroki zaotzen su ondoan, ongui yan eta edaten zutela, aberatz eta uros ciren hek. Arrast apalean, urrundik heldu ciren bi bideantek nahi izan zuten toki hartan guelditu, ceren eta hoztuak eta gosetuak baïtziren; hasi ziren athe bati yo? bertzeari yo, asko atheri haitu ziren yoka: nihork ez zituen utzi sartzen deusic ere ez zioten eman nahi yatera. Denetarik kasatuak, herriko zakhur guziak saïngaz guibeletik, bi arrotzek ez zakiten norat guan, noiz eta ere aurkhitu baïtziren herriaren azken mugan, guizon eta emazteki behar hekien etchearen aitzinean. Hekien oïhu saminak aditzearekin, guizona badoha leyhora eta emaztekia lasterka athearen idekitzerat.

Galdeguin gabe nor zitezkeyen, sarrarazten tuzte eta su ondoan yarrarazten; guero zituzten guziak eman ziozkaten yatera, esne eta gaztaina. Bainan orduan Yesu-Christo gure yauna, ezin hura bera zen yon doni Petrirekin, chutitu zen burua argui distirant batez inguratua eta erran cioten: Zuek pobreac zaïste eta onak; zuen haüzoak aberatsak dire, bainan gaïstoak. Yainkoaren yustizia eguin bedi. Orduan berean, lurra ikharatu zen, Yesu-Christo eta yon doni Petri ezeztatu ziren eta bi zaharrak belhaüniko erori ziren. Biharamun goizean, herrirat nahi izan zutenean guan, etcherik batere ez zuten ikhusi. Hek ziren lekhu guzia estalia zen ur hil batez. Ur hil hura da oraïno ikhusten Barbezan deitzen den herriaren eta Saint Bertrand de Commingeko hiriaren artean. Yende beharrak behar dire sokhorritu.

\section{oOo}

Tantugou guizon zahar bat da, oyhanetan gordetzen dena. Zaintzen ditu alhor eta pentzeak, nihork hetan ez dezan deusik ebats. Oguiak edo belharrak pikatuak direnean, Tantugouk ez du lorik batere eguiten, bethi erne dabila, naüsiak denak altchatu arteraïnokoan. Nondik aguertuko zaïoten Tantugou badaüde ohoinak, eta beldur horrek beiratzen tu ebastetik. Zinez, guizon ona da Tantugou, bainan guizon asprea. Asko zakien laborari batec zioten maïz bere semeeri Tantugou zela lurreko fruituen zaintzale hoberena.

Uztaileko arratsalde batez, ardi arthalde bat alan zagon mendi gaïn batean, artzaïna lokhartu zen, iratzartzearekin ikhusi zuen Tantugou ihesi oyhanari buruz zohala. Zelhaï eder batean higoal ezagutua izan zen Tantugou artzaïnez, harroka baten gainean etzana.

Haren beldur dire oraino haürrac gure tokietako herri zombaitetan. Muthiko batec edo nechka batek ez diotenean nahi amari obéditu, haürrari erraten ohi du amak: Goardia emac! Deitzen diat Tantugou eta, kuchean, ethorriko duc oyhanetik.

Instituteur: JB ${ }^{\text {te }}$ Marot, âgé de 40 ans, né à Hélette (Basses-Pyrénées) Coll.: Dufau Salvat, Maire. 
Baciren lehenago, Pyreneko herri batian guizon eta emaste batzu arras zaharrak. Etzuten etche ttipi bat, baratce bat eta behi bat baïcik. Baïnan, nahis beharrac ciren, laguntcen citusten bethi hec baïno beharrago cirenak. Egun batez, lurra elhurres estalia cen, eta horma hari harriac arrailtceko. Herriko yende guciak berotcen ciren, yaten eta edaten hari cirelarik; aberatx eta urus ciren. Ilhun heguian, bi piyayant, urrundic heldu cirenac, nahi izan zuten guelditu lekhu hartan, hotz eta gose cirelakotz. Yo zuten athe bat, guero bertce bat eta oraïno bertce batzuec: nihoc etcitusten sartzerat utzi nahi izan, ez deüsik yaterat eman. Kasatuyac orotarik, chakhur gaisto batzu ondotik, bi bidekariac etzaquiten norat yoan, heldu cirenean herriaren bururat, beharrak ciren guizon eta emaste heyen etchiaren ondorat. Hekien errenkurak aïntziarequin, senharra emantzen leïhoan hekien deïtceco eta emastia laster eguin zuen athiaren idekitcerat. Galdatu gabe nor ciren, sarrarazi zituzten eta yarrarazi su pasterrian; eskaïni ciozkaten yaterat esnia eta cembeit gastaïna, zuten guzia. Baïnan orduyan Salbatzaïlea -hura cen Yondoni Petrirekin- yequi zen, buruya distirant, eta erran zioten: "Zuek beharrak eta onac zarete; zuen haüzoac aberatxac eta gaïchtoac, zuzen dena eguin bedi!". Haïnbertcenarekin, ikharatu cen lurra, Salbatxailea eta Yondoni Petri ezetatu ciren eta bi zaharrak behaüniko eori. Biharamun goïzean, yoan nahi izan zirenean herrirat, cer iragan cen yakiteko, etzuten ikusi etche bihirik, heyen lekhuyan estainubat cen, egungo egunian han berian kaüsitcen dena Barbazango herriaren eta Saint Bertrand de Comminges deitcen den hiriaren artian.

Behar dire sokhorritu eskasian direnak.

\section{oOo}

Tantugou da zahar bat oïhanian gordetcen dena. Zaïntzen ditu landac eta sorhoac deus ez dezaten ebatx. Oguiak eta belharrac pikatuyac direnean, nausiak oro barnerat eman artio, Tantugou ez da lokhartcen; bethi atzarria dago. Hura aguertcen ikhus beldurrak daüska ohoïnak. Ona da, sinhetx zazue, baïnan basa da. Asko gausa zakien laborari batek bere semieri erraiten cioten maiz Tantugou cela segurki lurreko bihien zaïntzaïle hoberena.

Ustaileko arratxalde batez, arthalde bat alha cen mendi baten gaïnean; artzaina lokhartu cen: iratzartziarekin, ikhusi cin Tantugou oïhanerat ihesi zohala. Izan tzen orobat ezagutua artzaïnes, haran eder batian, etzana harroka baten gaïnian.

Egungo egunian oraïn, Pyreneko herri cembeitetan, haurrac haren beldur dire. Muthiko edo nechka ttipi batec, bere ama obeditu nahi ez duenian, erraiten dio: Kasu emac! Oïhu eguiten diat Tantugouri eta, cuchian, yinen duk oïhanetik.

Instituteur: Cazebonne, 28 ans, Espiute Coll.: Borotra, maire 
M. Duleau voudra bien faire aussi le travail de Halsou, en se faisant aider par l'instituteur.

\section{Barbazango iphunya}

Pireneetaco herrichka batean baziren lehenago guizon bat eta emazte bat, zaharkituac zirenac. Etzuten etche ttipi bat baizic, baratze bat eta behi bat. Bainan, zoin nahi beharrac baitziren ere, bethi laguntzen zituzten berac baino behartsuagoac zirenac. Egun batez lurra estalia zen elhurrez, eta horma hari zen harriac arrailtzeraino. Herrico guziac zauden berotcen, yaten eta edaten; aberats ziren eta zorionecoac. Ilhun nabarrian, bi bidezco, urrundanic heldu zirenac, nahi izan ziren herri hartan baratu, ecen hotz zuten eta gose ziren. Yo zuten athe batian, guero bertze batian, eta bai bertze batzuitan ere: Nehorc etzituin sartcera utzi eta etzioten yatera eman nahi izan. Alde orotaric guibelat egorriac, zakhur haserretsuac ondotic zarraizcotela, bi arrotzec etzaquiten norat equin, noiz ere aurkhitu beitziren herriaren mugan, guizon eta emazte behartsuen etchearen aintzinean. Aditziarekin heyen oihu minac, senharra athera zen leihora heyen deitcera, eta hunen emaztea yoan zen athearen idekitzera. Galdeguin gabe berei nor ziren, sarrarazi zituzten eta yarrarazi beren su bazterrian; guero eman zioten yatera esnia eta gaztena zembait, zuten guzia. Orduan Gure Yauna (ezen hura zen Yondone Petirirekin,) chutitu zen eta buruac miragarriki distiratzen zioela, erran zaien: "Zuec beharrac zirete eta onac; zuen auzoac aberatsac eta gaichtoac dira. Eguin beza Yaincoac zuzen zaiona!». Ordu berian ikharatu zen lurra, Jesus eta Yon done Petiri suntsitu ziren eta bi zaharrac erori ziren belhaunico... Biharamun goizian, nahi izan cirenian yoan herrira, yakiteco zer iragan othe zen, etzuten etcheric bat ere ikhusi: heyen tokian cen aintzira bat, zoina aurkhitcen baita gaurco egunian ere Barbazanco herrriaren eta San Betran Cominyeco hiriaren bitartian. Behar dira on-beharrac lagundu.

\section{Tantuguren iphunya}

Tantugou da guizon zahar bat oihanetan gordetzen dena. Beguiratzeintu lur lanthuac eta phentziac, ez dezaten hetaric deus ere ebats. Oguiac ebaquiac direnian edo belharrac ephainac; guzia yabiac bildua izan dadin artio: Tantuguc eztu loric eguiten, bethi iratzarria dago. Hura aguertcen ikhusteco beldurrac ohoinac guibelatcen ditu. Ona da bai, sinhets zazue, bainan basa da. Hainitz gauza zakien laborari batec erraiten zioten maiz bere semei, Tantugu zela eguiazki lurreco uzten beguirale hoberena.

Uztaileco hilabethian, arrats alde batez, arthalde bat alha zen mendi baten gainian; loac hartu zuen artzaina: iratzartzearekin batian, nabaritu zuen Tantugu, ihes zohala oihaneco alderat. Orobat ezagutua izan zen abere zainec haran edertto batian, harroca baten gainian lo zagola.

Gaurco egunian ere, Pireneetaco herri batzuetan, haurrac beldur dira harentzat. Noiz eta ere muthicotto edo nechca bat nahi eztenian iarri bere amaren erranera, amac 
erraiten dio. Beguirauc (beguirann)! Tantugu deitzen diat (dinat) eta berehala yinen duc (dun) oihanetic.

Instituteur: Catherine Lizon, S. M. Bathildis, Ste de M., qui a transcrit la transcription faite par M. Duleau

M. Duleau, 50 ans, né à Charritte de Bas Coll.: St Jean, Maire. 


\section{Barbazango iphunya}

Pireneetaco herrichka batian baziren lehenago guizon bat eta emaste bat, zaharkituac zirenac. Etzuten etche ttipi bat baizic, baratze bat eta behi bat. Bainan, zoin nahi beharrac baitziren ere, bethi laguntzen zituzten berac baino behartuagoac zirenac. Egun batez lurra estalia zen elhurrez, eta horma hari zen harria arrailtzeraino. Herrico guziac zauden berotzen, yaten eta edaten, aberats ziren eta zorionecoac. Ilhun nabarrian, bi bidezco, urrundanic heldu zirenac, nahi izan ziren herri hartan baratu, ecen hotz zuten eta gose ziren. Yo zuten athe batian, guero bertze batian, eta bai bertze batzuitan ere. Nehorc etzituen sartcera utzi, eta etzioten yatera eman nahi izan. Alde orotaric guibelat igorriac, zakhur haserretsuac ondotic zarraizcotela, bi arrotzec etzaquiten norat eguin, noiz ere aurkhitu baitziren herriaren mugan, guizon eta emaste behartsuen etchearen aintzinian. Aditziarekin heyen oihu minac, senharra athera zen leihora heyen deitcera, eta hunen emaztea yoan zen athearen idekitzera. Galdeguin gabe berei nor ziren, zarrarazi zitusten eta yarrarazi beren su basterrian; guero eman zioten yatera esnia eta gastena zembait, zuten guzia. Orduan Gure Yauna (ezen hura zen Yondoni Petirirekin) chutitu zen, eta burua miragarriki distiratzen zioela, erran zaien: "Zuec beharrac zirete eta onac; zuen auzoac aberatsac eta gaichtoac dira. Eguin beza Yaincoac zuzen zaiona!". Ordu berian ikharatu cen lurra, Jesus eta Yondoni Petiri suntsitu ziren, eta bi zaharrac erori ziren belhaunico... Biharamun goizian, nahi izan cirenian yoan herrira, yakitera zer iragan othe zen, etzuten etcheric batere ikhusi: heyen tokian cen aintzira bat, zoina aurkhitcen baita gaurco egunian ere Barbazanco herriaren eta San Betran Cominyeco hiriaren bitartian. Behar dira on-beharrac lagundu.

\section{Tantuguren iphunya}

Tantugu da guizon zahar bat oihanetan gordetzen dena. Beguiratzeintu lur lanthuac eta phentziac, ez dezaten hetaric deus ere ebats. Oguiac ebaquiac direnian edo belharrac ephainac, guzia yabiac bildua izan artio, Tantuguc eztu loric eguiten, bethi iratzarria dago. Hura aguertcen ikhusteco beldurrac ohoinac guibelatcen ditu. Ona da bai, sinhets zazue, bainan basa da. Hainitz gauza zakien laborari batec erraiten zioten maiz bere semei, Tantugu zela eguiaski lurreco uzten beguirale hoberena.

Ustaileco hilabethian, arrats alde batez arthalde bat alha zen mendi baten gainian, loac hartu zuen artzaina: iratzartziarekin batian, nabaritu zuen Tantugu, ihes zohala oihaneco alderat. Orobat ezagutua izan zen abere zainec haran edertto batian, harroca baten gainian lo zagola.

Gaurco egunian ere, Pirenetaco herri batzuetan, haurrac beldur dira harentzat. Noiz eta ere muthicotto edo nechca bat nahi eztenian iarri bere amaren erranera, amac erraiten dio: "Beguirauc (beguiraun)! Tantugou deitzen diat (dinat) eta berehala yinen duc (dun) oihanetic.

Instituteur: Duleau, 50 ans, né à Charritte de Bas Coll.: Cottenx, maire. 
Bacien lehenago Bortu herribatean senhar emaste batçu arras çaharrac. Deusere etçuten salbu etcholabat, baratcebat eta behibat. Bainan, cembat nahi beharrac ciren, bethi laguntcen citusten berac baino beharragoac. Egumbates, elhurres estalia çagoen lurra eta horma bat cen harriac erdiaraco cituena. Herrian denac su phasterrian çauden jan edanes. Aberax ciren eta dohaxu. Ilhuntcebatez, urrundanic heldu ciren bi arrotz batzuec nahi izan ciren herri hartan baratu, hotz eta gosse çutelacotz. Joïten dute athe batian, bertce bi, hiruetan, nehorc ez diote ideki nahi ez jatera eman. Batetic bertcera igorriric, çakhurrac jangaz ondotic bi arrotcec etzakiten norat itzul, noïs eta ere khaussitcen beitira senhar emaste behar heyen athean, herriaren buru buruan. Hekien auhenac adituric, leyhoan phasatcen da senharra, sarciten erraiteco, eta berehala emastia joan cen athearen idekitcera. Nordiren ere galdeguin gabe jarrarasten tuste su phazterrian, emaiten diotelaric dituzten guciac: esne chortabat, gastena cembaïtekin. Baïnan orduan, gure Salbatçailea - ceen hura cen jon doni Betirirekin, jeiki cen, buruac distiratcen dioela eta erraiten diote: Çuec beharrac cieste eta bihotx onac; çuen aüçuac aldiz aberax dire eta gachto: Eguin bedi Jaincoaren cucentassunari dohacona. Betbetan lurra ikaratcenda Jesus eta joandoni Betiri galtcen dira beguietaric, eta senhar emaste çaharrac erortcen belhaunico. Biharamunian badohatci herrira jakiteco eya cer guerthatu den, exdute etcheric ikhusten, bainan heyen orde ur hil haudibat, oraïno orai ere agueri dena Barbazaneco herriaren eta San Bertran-de-Commingeco hiriaren artian. Beharrac lagun ditçagula.

\section{Tantuguren erran çaharra}

Tantugu da guiçon çaharbat oyhanetan gorderic dagoena, çaïntux landac eta phentceac ohointçaric eguin estadin. Ustac eguinac direnian eta belharra ebakiric campoan oraino, Tantugu atçarria dago eta erne bethi. Hura aguertcen ikhus beldurrez urrundaüde ohoïnac. Ezta gaïchtoa, sinhexaçu baïnan bassa da. Asco çakien laborari batec bere semeï maïz erraïten cioten, Tantugu cela dudaric gabe lurrac emaiten dituen gauça gucien arthaçaleric hoberena. Ustaileco arrax aldibates ardi aldebat çagoen alhan mendibatean; artçaina lokhartcen da, eta iartçartcean ikhusten du Tantugou lasterca dohala oyhanian barna: bertce artçainec ere eçagutu cuten ibaï pullit batean harroka baten gainean cagoela etçanic. - Oraïno oraï ere haurrac haren beldur dire: muthico edo nechkabatec ezduenian bere amaren errana eguiten erraiten dio: emac cassu, oihu eguiten beitacot Tantuguri eta berehala jinen beita oyhanetic!

Instituteur: Carresson, Jean-Baptiste, né à Espelette le 27 Mars 1856 [?]. 
Bacen lehengo demboretan, Pyrénéataco herri batean, guiçon bat eta emazteki bat çoinac baitciren haïnitz çahartouac. Etçouten etche ttiki bat baïcic, baratce bat eta behi bat. Egoun batez lourra elhourrez echtalia cen eta horma eguiten çouen harrien arrailtceco guichan. Herrico yende gouciac berotcen çaouden, yaten eta edaten çoutelaric, aberatch eta ourouch ciren. Ilhountcean, bi piayant çoïnac heldou baïtciren ourroundic, guelditou nahi içan ciren toki hartan, ceren hotz eta goche baitciren: yo çouten athe bati, guero bertce bati, eta bertce achkori ere: nihorc et citouen chartcerat outci nahi, ez eta ere deouchic yaterat eman nahi içan. Toki goucietaric hachatouac, yarraikiac chakour gaïchtoez, bi arrotz hec et çakiten norat goan, noïz eta ere aourkitou baïtciren herriaren mougan, erromech ciren guiçon eta emazteki horien aïntcinean. Aditcearekin hekien plaïnouac, chenharra eman cen leïhoan hekien bere ganat deitceco eta haren andrea goan cen lachter athearen idekitcerat. Hekieï galdetou gabe nor ciren, chararazi citouzten eta yararaci beren chou bazterrean; guero, cerbitçatou ciozkaten echnea eta cembeit gaztaïn, citouzten gouciac. Baïnan ordouan, Goure Yaouna -houra cen Yondoni Petrirekin- choutitou cen, bouroua gouciz dichtillanta çouelaric eta hekieï erran cioten: "Çouec pobreac çaizte eta bihotz onecoac; çouen haouçoac dire aberatch eta gaïchtoac. Yaincoaren youchticia eguin bedi!". Dembora berean lourra ikharatou cen, Yechuch eta Yondoni Petri chounchitou ciren, eta bi çaharrac belhaounico erori ciren... Biharamoun goicean, goan nahi içan çoutenean herrirat yakiteco cer pachatu cen et çouten etcheric ikhouchi, hekien lekhouan, bacen ouril bat, egoun oraïno Barbazango herriaren eta Saint-Bertrand-de Comminges deîtcen den hiriaren artian aourkitcen den houra bera.

Behar dire lagoundou malourouchac.

\section{oOo}

Tantugou da çahar bat oïhanetan gordetcen dena. Çaïntcen ditou landac eta chorroac, amorea-gatic eta deouchic ez deçaten ebatch. Noïz eta ere oguiac edo belharrac pikatouac direnian, denac naouchiaz bordan chartouac içan artian, Tantugouc ez dou lo eguiten, bethi atçarria dago. Haren aguertcen ikhouchteco beldourrac guibelatcen ditou ohoïnac ebachtetic. Bihotz onecoa da, chinetch-çaçoue baïnan chalbaya ere. Gaouça haïnitz çakizkan laborari batec erraiten cioten maïz bere semeï Tantugou cela, seguric, lourreco frouitouen çaintçailleric hoberena.

Ouztailaren ilhabeiteco arratch-alde batez, ardi arthalde batec alatcen hari cen mendi baten gaïnean. Artçaïna lokhartou cen: atçartcearekin, ohartou cen Tantugouc ihech egiuten çouela oïhanerat. Orobat, eçagoutoua içan cen acienda çaïntçaïlez mendi-tarte pouillit batean, etçana celaric arroca baten gaïnean.

Egoungo egounean oraïno Pyreneetako cembeït herrietan, haourrac hartaz beldour dire. Noïz eta ere mouttico ttiki batec edo nechka ttiki batec ez dionean obeditou nahi bere amari, hunec erraiten dio: Contou egui çac hitaz! Deitcen dout Tantugou eta couchian ethortcerat doha oïhanetic!

Instituteur: Darré, L., 35 ans, né à Assat, près Pau. Coll.: Soudre, Pierre, Larralde Dque, Duhart. 
Baciren lehenago Pyrenetaco herri batean guizon eta emazteki bat arras zaharrac. Ez tzuten etche ttipitto bat, baratce bat eta behi bat baicic. Bainan, nahiz hain beharrac ciren, socorritcen cituzten bethi hec baino beharrago cirenac. Egun batez lurra elhurrez estalia cen, eta harrien arrailatceco horma hari cen. Herri hartaco yende guciac hari ciren berotcen, yaten eta edaten; aberas eta dohatsu ciren. Ilhuntcean bi piyayant, urrundic heldu cirenac, nahi izan ciren lekhu hartan guelditu, ceren hotz eta gose baitciren; yo zuten athe bat, guero bertce bat eta oraino bertce batzuetan: nehorc ez cituzten sartcerat utci nahi izan, eta nehorc ez ahamen bat eman nahi catu. Orotaric igorriac, zakhur gaitzac ondotic, bi arrotzec ez tzakiten norat yuan, causitu cirenean herriaren ichkinean, guizon eta emazki behar hein bortan. Aditcearekin hein auhenac, senharra eman tcen leihoan deitceco, eta haren emaztea yuantcen zaluki athearen idekitcerat. Galdatu gabe nor ciren sar eta yar araci cituzten su basterrean, guero eman cioten esnea eta zombait gaztena, zuten gucia. Bainan orduan Jesus Gure-Yauna, -ecen hura cen Yondoni Petrirekin- chutitu cen, burua oro dirdiran, eta erran cioten: "Zuec beharrac eta onac zarete; zuen hauzoac aldiz aberasac eta gaichtoac. Yaincoaren justicia eguin bedi!". Berehala lurra ikharatcen da, Jesus eta Yondoni Petri suntsitcen dire eta bi zaharrac emaiten belhaunico. Biharamun goicean herrirat yuan nahi izan cirenean ikhusteco cer pasatu cen, etcen guchihago etcherric batere: heien lekhuan, bacen aincira handi bat, causitcen dena oraino egun Barbazanco herriaren eta San Bertrand-Commingeco hiriaren artean.

Behar dire dohacabeac lagundu.

\section{oOo}

Tantugu da oihanetan gordetcen den zahar bat. Beiratcen ditu landac eta penciac deusic ebasterat ez uzteco. Oguiac eta belharrac picatu direnean, oro nausiac barnerat eman nahi artio, Tantugu ez da locartcen, bethi erneric dago. Hura ikhus beldurrez ohoinac urrun daude. Ona da, segurki, bainan basa. Hainitz gauza zakizcan laborari batec cion maiz bere semieri Tantugu cela segurki lurreco fruituen zainzaile hoberena.

Uztaila hilabeteco arratsalde batez ardi arthalde bat alha zagon mendi baten gainean; arzaina lokhartu cen: iatzarcearekin, ikhusi zuen Tantugu ihes zohala oihaneco alderat. Ezagutu zuten bardin zainzailec haran pullit batean, etzana zagola harroca baten gainean.

Oraino egun, Pyrenetaco herri batzuetan, haurrac haren beldur dira, mutico edo nechca batec ez duenean bere ama obeditu nahi erraten dio: Guardiemac! Tantuguri oihu eguinen zioat!

Instituteur: Larralde Pierre, 5 X ${ }^{\text {bre }} 1827$, St-Jean Pied-de-Port. Coll.: Ibarboure, Bernard - Etcheverry, Jn B. 
Bazen lehenago Pyrénétako herri batean gizon bat eta emaztebat arras zaharrak zienak. Ez zuten etche ttipibat, baratzebat eta behi bat baizik. Bainan nahi bezen pobre izan ziten, laguntzen ziuzten bethi berak bainago pobreago zirenak. Egun batez, lurra estalia zen elhurrez eta horma eguiten zuen harria erdikatzeko heguinean. Herriko jende guziak berotzen zien, jaten eta edaten zutelarikan; aberatzak eta irutzak ziren. Ilhun bazterrean, bi pieyjant, urrunetik heldu zirenak nahi izan ciren arratztatu toki hartan zeren eta gotze eta hotz baitzuten. Jo zuten bortha batean, guero bertze batean eta oraino bertze batzuetan: nehok ez ziusten sartzera utzi nahi izan ez eta deusik eman jaterat. Orotarik kasatuak, chakhur furius batzuz seguituak, bi estranjerek ez zakiten norat joan, noiz eta ere kausitzen baitira herriaren azken buruan guizon eta emazte pobre heyen etchiaren aitzinean. Aditurik heyen pleynuak, senharra eman zen leyhuan ekharrarazteko eta haren emastea johan zen fite bortharen idekitzerat. Galdegin gabe nor ziren, sarrarazi ziusten eta jarrarazi beren supasterrean; guero çombait gastena eta esnia zerbitzatu zioten, zuztenak oro. Bainan, orduan Gure Jainkoa -huranzen, San Pierresekin - chutitu zen burua oro dichtiran eta erran zakoten: "Zuek pobreak zirezte eta onak; zuen auzoak aberatzak eta gaichtoak dira. Jaincoaren juztizia egina izan dadin."Ordu berian, lurra ikharatu zen, Jeuss eta San Pierres etsestatu eta bi zaharrak erori ziren belhaunika. Biharamun goizean, joan nahi izan zirenean herrirat, jakiteko zer pasatu zen ez zuten ikhusi etche bihirik: heyen tokian esteynu bat zen oraino egun kausitzen dena Barbazaneko herriaren eta San Bertrand-de-Commingeneko hiriaren artean. Lagundu behar dira malerusak.

\section{oOo}

Tantugou oyhanetan gordetzen den chahar bat da. Guardiatzen ditu landak eta pentzeak deusik ez dezaten ebats. Ogiac phikatuak direnean edo belharra ebakina oro sarthuak izan artino nausiaz, Tantugouk ez du batere loik egiten; bethi beilan dago. Hura ager dadein beldurrak atchikitzen ditu ohoinak. Ona da, sinhez zazu, bainan salbaia da. Asko gauza zakien laborari batek erraiten zuen usu bere semeri Tantugou zela, segurki, lurreko frutuen guardiatzalerik hoberena.

Eriaro hilabateko aratsalde batean, ardi tropabat alhatzen zuen mendi baten gainean; artzeina lokhartuzen: iratzarzean ikhusi zuen Tantugou espakatzen zela oyhanari buruz. Izantzen bardin ezagutua artzainez chola pullit batean, etzana harroka baten gainean.

Oraino egun, Pyrénétako herri batzuetan, haurrak horren beldur dira. Noiz eta ere muthiko ttipi batek edo neskatoko ttipi batek ez baitu nahi bere amari obeditu, amak erraiten dako: Gaurdia emak hiri! Oihu eguinen diat Tantugouri eta, ichtantian, jinen duk oyhanetik.

Instituteur: Etchevers, 27 ans, né à Briscous (Basses-Pyrénées) Coll.: Larre, Jean - Broussain, Louis. 


\section{Barbazaneco istoria}

Bazen lehenago Pyrénees taco herri batian guison bat eta emaste bat biciki çaharrac. Ez çuten etche ttipi bat, baratze bat eta behi bat baïcik. Bena haïn pobre içanikan ere zokhorritzen zousten bethi hec beno pobriago zienac. Egun batez lourra elhourez koukoutia cen eta kharrougna ari zen harrien erdiatzeco gisan.

Herrico jende gouciac berotsen ari ziren jatian eta edatian; aberatz eta irous ciren. Ilhountsian bi pidejantec, zoïnac ourhountic heldu baïtciren, nahi izan zouten guelditou toki hartian, cendaco eta hotz baïtciren eta gosetiac; jo çouten bortha batian, guero bertse batian eta beste frangotan; nehorec ez citousten nahi oukhan outsi sartcera, ez eta deüze eman jatera. Orotaric casatiac, chakhurrez seguitiac, bi estranjerrak ez zakiten noat jouan, noïz eta ere khaüsitou baitziren herrian basterian, guison eta emazte pobre hen etche aitcinian.

Hen plegniac entzounik, zenharra jarri cen leyhouan, her oïhou eguiteco eta emastia jouan cen fite bortharen zabalcera.

Galde eguin gabe nor ciren sar araci citousten eta jar arasi sou bazterrian; guero eman zeen jatea esnia eta gaztegna cembait, zouten goucia. - Baïnan ordian goure Yinkoa -çoina baitcen Yondane Petriekin, - choutitou zen buria argui, arguia eta erran zeen - "Ciek pobre ciste eta hon, cin aïzouac aberats eta gaïstouac dira. Yinkouaren joustizia eguina izan dadiela. Ordu berian lourra ikharatou zen, Yésous eta Jondana Pethri galdou ciren eta bi zenhar emazte hourac eman cien belhaounico. Bihamen goïcian, noïz eta ere jouan nahi izan beitçouten eliçatherat jakiteco cer passatou cen etzouten ikhouci etche bihi bat ere: hen lekhian bacen phoutsou handi bat, zoïn khaüsitzen baïta oraïno egun Barbazaneco herrian eta St Bertrand de Commingeco hirian artian.

Lagoundou behar da pobriac.

\section{Tantugouren istoria}

Tantougou da guizon zahar bat oyhanetan gordatzen dena. Zaintzen ditou alhorrac eta phensiac, deüzic ebatz ez dezaten.

Noiz eta oguiac mostiac baïtire edo belharra ebakia, hec denac naousiac sar artino, Tantougou ez da loc hartzen, gouaitian dago bethi. Haren aguertzen ikhousteco atchikitzen ditou ouhougnac.

Hona da baina sabaje da.

Laborari batec zoinec beitzakien biciki gaousa, erten cin ousou bere semer Tantougou zela seourki lurreco fruten zain zale hobia. 
Oustarilaco atzalde bates, azinda multso bat alha zagon bortou batian; arzaina loc hartou zouen; iratzartzian apercebitu sin Tantougou ezkapatzen cela oyhanerat bourous.

Giza berian ezagoutia izan cen bertze artzaïnes etzanic phegna batian.

Oraïno egoun Pyrénées taco herri batzoutan, haourrac haren beldour dira.

Noïs eta mouthico ttipi batec edo neskato ttipi batec ez baitou obéditou nahi bere amari, hounec erten dero- "Gourdia emac! oyhou eguiten doat Tantugouri eta istantian jnen duc oyhanetic.

M. Achiary, 22 ans, né à Esquiule Coll.: Guissagaits 


\section{Barbazaneco berrichaharra}

Baçitçoun behin bortietaco herri batian guizon bat eta emazte bat biciki çaharrac. Ez cicin etche thipi bat, batce bat eta behi bat becic. Bana haïn probe içanic ere, laüncen citcin beti bera bano probiagoac. Egoun batez, lourra coucoutia çouçoun elhourrez eta hormatcen cicin harriac ourratceco heïnian. Herrico yende gouciac berotcen ari citçoun yaten eta edaten çoutelaïk. Aberatsac citçoun eta ouros. Ilhounarrian, ourrounetic heldu cin bi piayyantec nahi ouhkan cicin guelditou lekhou hartan, ceren gose eta hotz baïtçouten. Yo cicin bortha bati, guio beste bati, eta beste batcouer oraino: nehoc ez citcin sartcera outci nahi oukhan, ez eta ere eman yatera. Kasatiac orotan, seguitiac chacourrez, bi guiçonec ezçakizin noat youan, noizeta ere kausitou baitciren herriaren bourouan, probe cin guiçon eta emazte hen etchiaren aitcinian. Intçoutiarekin hen arrangourac, senharra yarriçouçoun leïhouan, her oïhou éguiteco, eta emaztia youana çouçoun fite bortharen idekitcea. Yarraci citcin beren soupazterrian, galdeguin gabe nor ciren; guero eman cecin esnia eta çombeit gaztina: çouzten gouciac. Bana ordian Goure-Yincoa -houraçouçoun Yandone Petrirekin- choutitouçouçoun, bouria distirant eta erran cecin: "Cic probiac cizte eta hounac; cin aücoac abératsac ditouçou eta gaïchtouac; Yaincoaren yusticia eguin daïlala.". Behala lourra ikharatu çouçoun; Jeus eta Yandone Petri gorde citçoun eta bi çaharrac erori citouçoun belhaünico. Bihamoun goïzian, herrirat youan nahi oukhan çoutenian ikousteco cer guerthatou cen ez cicin ilhousi etcheric bate. Hen lekhian baçouçoun acekiabat, Barbazanen eta Saint-Bertrand de Commingen artian kaüsitcen den hori bera.

Behar dira sokorritu malourosac

\section{Tantougouin berrichaharra}

Tantugu douçou oyhanetan gordatcen den yende çahar bat. Béyratcen tici landac eta penceac han ez deçaten deusere ebats. Oguiac phicatiac dinian, eta belharrac ebakiak, nagousiac gouciac etcherat bildou artino, Tantugouc ez dici lo eguiten; beïlatcen dici bethi. Hain atheratcen ikousteco beldourrac atchikitcen tici ohoïnac. Houna douçou, sinetsacie, bana basa douçou. Laborari, anhitz gaüça çakien batec, erten cicin ardoura beré semer Tantugou cela segourki lourreco fruitouen beïraçaleric hobena.

Ouztaïleco egouerdi ondo batez, ardi moulço bat alha çouçoun, mendi baten gainean, arcaïna lokhartou çouçoun: Yatçartcearekin, ikhousi cicin Tantugou espacatcen cela oïhaneco alderat. Berdin eçagoutoua içan çouçoun artçaïnez mendia arte poillit batean, etçanic harroca baten gaïnean.

Ono egoun, Bortietaco herribatçouetan, haürrac beldour touçou haren daco. Mouthico thipi batec edo nechcato thipi batec obéditou nahi ez dinian bere amari, hounec erten dacoçou: "Kasouiçac hiri, deithatcen diat Tantugou, eta behala ynen douc oyhanetic"

1. En s' adressant à la fille: "Kasouiçan hiri, deithatcen dinat Tantugou eta behala ynen doun oyhanetic." 


\section{Barbazen én historioa}

Bassèn lénénago Pyrénées herri batian guiçon bat éta émazté bat çoinac bissiki sahartiac baitcirén. Etsoutén étché ttipi bat baïssic, baratcébat éta béhi bat. Baïnan bissiki pobré issanic èrè lagounsen sitoustén hec baïno pobrio siénac. Egoun batès, lurra coucoutia sén élhourrez, éta carrougna ari sén harri én haoustéco poundourano. Herrico yéndé goussièc bérotsen sièn, yatian éta édatian; abérats sién eta irous. Ilhounsian, bi boyajurrac, çoïnac heldou beïtsien ourrounétic, nahi oukhen soutèn baatou lekhou hartan, céren beïtsouten gossia éta hotsa; yo soutén bortha bat, guèro bestébat éta oano bésté batsou: néhorc étsouten nahi oukhen outsi sharcèa, ez éta éré néhorc éman déouz yatéra. Orotaïc khassatiac, chacur gachtouès séguitiac, bi estranyèrrec espeïtsakiten noat youan, noïz éta éré guerthatou béïtsien herri én bourian, guiçon éta émasté pobré héen étché én aïtsinian. Entsoutia ékilan héen plégniac, sénharrac yarri sén leïhouan her oïhou éguitèco, éta harén émastia youan sén fité bortha én sabaltsèra. Galdéguin gabé nor sirén sarrhassi zitién éta yarrhassi sou én oundouan; guèro cherbitchatou sérén ésné eta gastagnac, sousténac oro. Baïnan ordian, Gouré yincouac houa sén youandéné Pétrirékin, yeîki sen, bouria arras argui éta érran zén: "Siéc, pobré sisté éta houn; sién aoüssouac abérats sou éta gachtouac. Yincoua én youstissia éguin dessala!". Mémentian lurra ikharatoussén, Yésous éta Youandéné Pétri parthitoussiren éta bi saharrac érorissien belhaoünico. Bihamoun goïssian, noïz éta éré nahi ouhken beïtsoutén youan hérrira, yakitèco ser passatoussén, étsissien ikhoussi étché bat èré::héen lékhian bassén poutsoubat, çoïna guerthatcen beïta égounéco égounian éré Barbazanéco herriaen éta Saint-Bertrand-deCommingéco hiria én artian. Béharda lagoundou malérousac.

\section{Tantugou én historioa}

Tantugouc saharbat da çoïna gordatsen beita oïhanetan. Béguiratsen tou alhorrac éta pénsiac ébats éz dessatén déouzere. Noïz été éré oguiac pikatiac beitira édo belharra ébakia, naüssiac goussiac éréman artino. Tantugou etsou lokhartsen, bethi goardiatsen ditou. Ohouinac ez dira ibilten hac ikhous beldurres. Houna doussou, sinhetsassou, baïnan bassa doussou. Laborari batec coïnac baïtsakien hanits gaüssa erraiten sien oussou béré sémér Tantugou cèla, doudaric gabé, lurréco frouitou béguirassalé hobéréna.

Oustailleco atsaldé bates, ardi saldo bat alhatsen sén méndy bat en gaïgnian, arshaïna lokhartou sen: iatsartsia ékilan, ikhoussissien Tantugouc youaïten sèla oïhanéako aldéat. Berdin essagoutia issan sén oulhaïn bates ordoki eder batian, etsanic arrocabat en gaïgnian.

Oraïno égoun, Pyrénéco herri batsoutan, haürrec beldour dira harèndaco. Noïz éta éré mouthico ttipi batec edo neskato ttipi batec obéditou nahi estiénian béré amari, erraiten daco: Khassou éguissac! Ekharraassico diat Tantougou, éta bérhala yinen douc oïhanétic.

Lavie, J., 27 ans, né à Burgaronne Coll.: Officialdéguy et Etcheberry 
92

Commune: Bunussé (Bunus.)

Hameaux: -

Quartiers: -

Cours d'eau: Ourhaitça (Bidouze); Hosta houra (Ruisseau de Hosta). Ibarla houra (Ruisseau d'Ibarrolle). Abouns erreca (Ruisseau d'Abons). Ithurraldeco erreca (Ruisseau d'Ithurralde).

Sources: Larcochéco ithourria (Source de Larcoche). Larratéco ithourria (Source de Larrats)

Fontaines: Urrutigaraïco ithourria (Fontaine d'Urrutigaray), Larcochéco ithourria (Fontaine de Larcoche), Ibarnéatcineco ithourria (Fontaine d'Idiart)

Collines: Bunus mendy (Colline de Bunus), Ibar mendy (Collinde d'Ibarre), Darthia (colline de l'église)

Coteaux: Gaïnsabalé (coteau sur la colline de Bunus), Haran érréca buria (coteau sur la colline de l'église).

Vallées: Bunusséco ordokia (vallée de Bunus).

Bois: Hiru haritsac (les trois chênes), Larcoche (Larcochéco oïhana).

Rochers (Harrocac).

Bornes: Au nord, Larceveau; à l'ouest Juxue; à l'ouest, Ibarrolle et au sud, St-JustIbarre. 
Basen lehenoo bortuetaco herri batian guizon eta emasteki bat biziki çaharrac ziinac. Etzuten etche tipi bat, baatze bat eta behi bat beïzic. Baïnan, haïn beharrac içanaatic ere laguntzen ziousten bethi hec beno beharrogouac ziinac. Eoun batez, lourra estalia zen elhourrez, eta horma aïzen harria arrailatzeko heïnian. Herrico yende gouziac beotzen ziin, yaten eta edaten zoutelaï, ouros eta abaats ziin. Ilhoun dirian, bi piaiant ourroundic heldou ziinac nahi oukhan zouten guelditou toki hartan, ceen eta gose eta hotztuac beïtziin; yo zuten borthaatian, gueo bestian eta besteatzuetan oono: nehoc etzitiin sarcea outzi nahi içan ez eta ere yeous eman yatea. Ootaïc khasatuac, bortchatuac chakhourrez guibeletic, bi arrotzec etzakiten noat youan; guerthatou ziinian herriaan basterrian, guiçon eta emazte beharrac ziin heen etchiaan aïtzinian. Heen souspirac aïtziaakin, senharra youan zen fite borthaaan idekitzea. Heer galdein gaa nor ziin, sarraazi ziousteten eta yarraazi souphasterrian; gueo eman tzeeten esnia eta çoïnbeit gaztena, souten goucia Baïnan orduan - Goue Yaouna, houa zen Yondoni Pétriikin- yeïki zen bourua distirant distiranta, eta erran tzeen: "Ziic, beharrac eta onac ziizte, ziin aouzouac abaats eta gaïchtouac dia.Yinkhouaan nahi eïn dailala!". Berheala lourra ikharatou zen, Yesous eta Yondoni Petri esdestatu ziin eta bi zaharrac belhaouco erori ziin... Bihaamoun goïzian, nahi oukhan zoutenian herriaat youan, yakiteco zer phasatou zen, etzouten ikhousi etcheïc batee. Heen tokian bazen touloumio bat (our geldi handi bat) houa oono eoun khaousitzen dena Barbazaneco herriaan eta Saint-Bertrand de Commingeco hiriaan artian. Behardia lagoundou malouousac.

Note explicative pour pour prononcer les lettres $z$, s y et $\mathrm{t}$

Prononcer la lettre $z$ comme l's en français

s sans appuyer la langue sur les incisives inférieures presque comme le $j$

y le bout de la langue appuyé sur les incisives inférieures et détachant la langue du palais

$t$ de tipi comme $y$ fort

oOo

Tantugou da chahar bat gordatzen den oïhanetan. Tzeintzen tou landac eta phentziac hetan ez dezaten yeous eats. Oguiac eta belharra eakiac diinian, naussiaz denac sarthuac içan artino, Tantugou ezdao lo; bethi iatzarriic doo. Haan aguertziaan beldourrac atchiitzen tou ohoinac. Ona da, sinhets zacii, baïnan basa da. Laborari batec zakiinac asko gaouza irten ziin ardoura bere semer Tantugou zela seourki lourreco frouituen tzeintzaleïc hobeena.

Oustaileco aatsalde batez, ardi arthalde bat alhatzen zen mende baten gaïnian: artzaïna lokhartou zen: iatzarziaakin, ohartouzen Tantugouri ihes yohan oïhanaï bourouz. Içan zen ere berdin artzainez ezagoutua errephea pollit batian harroca baten gainian 
Oono eoun Bortuetaco herri zoïnbeitetan haourrac haan beldour dia. Mouthico edo nechcatoco tipi batec ezdiinian obeïtou nahi bere amari, hounec erten daco: "Khasou man hire! Ekharraaazten diat Tantugou eta, berheala yien douc oihanetic

Dans la localité le basque est prononcé comme ci-dessus mais pas orthographié ainsi.

Gastellu, 32 ans, né à Hélette 


\section{Barbazaneco gerthacaria}

Bacitzun lehenago Pyreeneco herri batian gizon bat eta emazte bat çoinac baitciren arras çahartiac. Etcicien etche ttipi bat bat, baratce bat eta behi bat baicic. Bainan, nahiz arras beharrac ciren, bethi laguntcen citcien hec beno beharrago cirenac.

Egun batez, lurra elhurrez estalia çuzun, eta kharroiña ari harriac erdiratceco phundian. Herriko yende guciac berotcen ciaudezun, yaten çutelaric eta edaten; aberatz citzun eta uros.

Ilhuntzian, bi bidayant, çoinac heldu baitciren urrunic, nahi izan citzun gelditu lekhu hartan, ceren eta hotzac eta gosiac baitciren; yo cicien bortha batian, gero beste batian eta beste askotan oraino: nehoc ere etcitzien nahi ukhan utzi sartcerat ez eta ere eman yaterat. Kasatiac orotaric, eta segituac chakhur oldartu batçuez, bi arrotzec etciakicien norat yuan, noiz eta ere khausitu baitciren, nola etzakitela, herriaren basterian, gizon eta emaste behar heien etchearen aintzinian. Entzutiarekin heien plegnuac, senharra athera çuzun leihora heier oihu eguiteco, sartciten eta haren emastia aldiz yuan çuzun laster bortharen idekhitcera. Batere galdegin gabe nor ciren, barnerat sarraraci citzien eta yarraraci beren suphasterrian; gero zutenetic eman ciecien, esnia eta çombait gastaña. Bainan ordian, Jesus Jauna - ecic hura çuzun Jondoni Petrirekin - chutitu çuzun: ciec, beharrac eta hunac ciezte, cien auzuac aldiz aberatzac eta gaichtuac. Jaincoaren yusticia egindadiela!". Ordu berian lurra ikharatu çuzun. Jesus eta Jondoni Petri itzaldu citzun eta bi çaharrac erori belhaunico... Biharamun goician, nahi içan çutenian yuan herrirat, yakiteco cer pasatu cen, etcicien ikhusi etche bat ere, heien lekhian, baçuzun hur zabal geldi bat, çoina egungo egunian oraino khausitcen baita Barbazaneco herriaren eta SaintBertrand de Commingeco hiriaren artian. Behar dicie lagundu malhurosec eta beharrec.

\section{Tantugouin gerthacaria}

Tantugou duzu gizon çahar bat, çoina gordatzen baita oihanetan. Saintzentzi alhorrac eta phentziac amoregatik eta ez deçaten hetan deusere ebatz. Noiz eta ere ogiac phicatiac baitira edo belharra ebakia, handic eta nausiac oro barnerat sarthu artio, Tantugouc etci loik egiten; bethi zain dioçu. Ohoinac etzu menturatcen hura ager beldurrez. Huna duzu segur bainan basa. Anhitz gausa zakien laborari batec bere semer ardura erraiten ciezun Tantugou cela segurki lurreco fruituen zaintzaleric hoberena.

Arraxalde batez, ustaila çuzun, ardi saldo bat alhatcen zagoelaric mendi batian, artzaina lokhartu çuzun: iratzartciarekin ohartu çuzun Tantugou lasterca sohala ihes oihanerat. Beste artzain batzuec ere ikhusi cicien mendi arte pollit batian, harroca baten gainian etzanic.

Egungo egunian oraino, Pyreneeco çombait herritan, haurrac haren beldur tutzu. Noizeta ere muthico ttipi batec edo nechcato ttipi batec ez baditu amaren maniak egin nahi, erraiten diacozu: Gogo eman gero hiri! Oihu egiten dacoat Tantugouri, eta, berheala yinen duc oihanetic!

Sagardoy, âgé de 27 ans, né à Lacarre Cool.: Uhart et Chourrout, propriétaires 


\section{Barbazanen ichtorioua}

Bacitzoun léhénoo, Pyrénétaco hérri batian, guiçon bat éta émazté bat biciki çahar ciénac. Etcicién étché ttipi bat bécic; baatcé bat, éta béhi bat. Béna haïn pobré içanaatic, sjokhorritcén citcién bérac béno pobrio ciénac. Egoun batéz, lurra elhurrez coucoutia çouçoun, éta kharrogna azkarki ari. Hérrico yénde gouciac berotcén ari citçoun yaten éta édatén ari ciélaïc, abéatj éta iruj citçoun. Ilhountcian, bi voyajur ejtranyer ourrounic heldou ciénac, nahi oukhan cicien lékhou hartan guélditou gosjé éta égarri ciélacotz: yo cicien bortha batian, guéo bejte batian, eta bejte batçoutan oono. Néhoque ezcitcien jartcen utci nahi oukhan éta ez deouje yatéa eman. Gucietaïc kasjatiac, chakhour gaitz batzouz sjeguitiac, bi estranyerrek ezciakicien noat youan, noiz éta ére heldou baïtcien herriaïn bouroura guiçon éta émazté pobré héen étchiain aiitzinea. Héién plégniac intçoutian sjenharra leihoa yalqui çouçoun éta émaztia youan çouçoun fité borthaïn saaltcéa. Galdin gabé norcien yarraaci citcien éta yarraaci béren southonduan, guéo cerbitchatou ciécién oguia éta çombaït gaztagna, ciouztenac oro. Béna ordian Guré Yaüna, houa çouçoun, yandone Petriikin - yéiki çouçoun bouria argui arguia, éta érran ciéçoun: Ciéc pobriac cizté éta hounac, cién aouçouac abéatjac tçou éta gaïchtouac. Yincouaïn yousticia in dailla. Béhala lourra ikharatou çouçoun. Yéjus éta Yondoni Petri esjtali éta bi çaharrac belhaunico érrori bihamoun goïcian herrian cer phasjatu... ikhousjtéa, youan nahi oukhan çoutenian, étcicien ikhousji étchéic bate; hén plaçan baçouçoun phoutçou haoundi bat, égoun oono Barbazaneco herriain éta Sjan Bertran Comminjeco hiriain artian khausjitcen déna. Béhar dira malérousjac laoundou.

\section{Tantugouin ichtorioua}

Tantugou guiçon çahar oỉhanétan gordatcén den bat douçou. Beïratcentci alhorrac éta phentciak déousjé éz déçaten eatj. Oguiac phicatiac edo belharrac ebakiac diénian, oro nausjiétaz érrétiatouic içan artio Tantugouc étci loïc iten. Béthi beillatcén dioçou. Haïn aguertcen ikhoujteco beldourrac atchikitcentci ohognac. Hunaouçou sjinetjacie, béna sjalbayaouçou. Laborari, anhitz gaouça çakien batec, érraiten cicin ardoura béré sjémer; Tantugou cela sjéourki lourréco fruitouen beïratcaleïc hobéena. Uztaillaco-atjalde batez acinda sjaldo bat alha çouçoun mendi batian; artzagna lokhartou çouçoun. Iatçartcian, ikhousji cicin Tantugou oïhaneat bourouz ejpacatcen céla. Guija bérian éçaoutouic içan çouçoun artçagnez planada batian harroca baten gagnian etçanic. Egoun oono Pyrénétaco herri çombéitetan haourac haïn beldourtçou. Mouthico ttipi édo néchcato ttipi batec ez badou amari obéitou nahi erraiten diacoçou: Gouardémac hiri! Oïhuïten diat Tantugouri éta béhala yiten douc oïhanétic.

M. Chaho, 21 ans, né à Arhansus U se prononce ou. 
Behin batez bacitzun Españako fonteraco (Pyrénées) herri batian, senhar-emazte batzu arras zahartiac. Etzicien etche ttipi bat, baratze bat eta behi bat beizic. Bana nahiz berac beharrac ziren, sokhorritzen zititzien bethi berac bano beharrauouac.

Egun batez, bazter guciac elhurretan zitzun eta horma bat gaitza zuzun. Nola herri hartaco jendiac ontsa aberats eta uros beitzien, beren suphazterretan ziaudezun jan eta edanez. Ilhountzian bi bidayant urhunic heldu zirenec nahi ukhan zizien herri hartan gelditu, aloyatu, zeren hotzac hila eta gose errabiatiac beitzien. Ibili zitzun bortha bat jo, beste bat jo, nehoc etzitzien sartzeat utzi, ez eta ere deus jateat eman nahi ukhan. Lekhu gucietaric casatiac, zakhur furiosen ondotic mehatchuz... bi campotiar gaichuec etzakicien noat juan, causitu zienian herriaren bazterrian, aiphatu senhar emazte behar heyen etchiaren aitzinian. Heyen pleñiac entzunic, senharra jalgi zuzun leihorat oihu iteco barnerat sartziten, eta emaztia juan zuzun laster bortharen idokitzeat. Galdegin gabe ez nortzien ez zertzien, sar-arazi zitzien suphazterrian jar arazi eta esne eta gaztaina jaterat: etzicien besteric.

Bainan ordian, Gure Jauna - hura zuzun Jondoni Petrirekin- chutitu zuzun buria distiretan eta erran zizun: Ziec beharrac ziezte eta hounac, eta aldiz zien auzuac aberats ditzu eta gaichtuac. Jaincoaren justizia eguina izan bedi! Berehala lurra ikharatu zuzun. Jesus eta Petri bichtatic galdu zitzun eta bi zaharrac belhaunica pharatu zitzun.

Biharamun goizian nahi ukhan zizien jakin zer gerthatu zen juan ziren herrirat eta etzizien etcheric batere ikhusi: heyen lekhian osin bat zuzun, egun oraino causitzen dena Barbazeneco herriaren eta Cominseco herriaren artian.

Ichtorio horrec eracusten du behar diela malurosac lagundu.

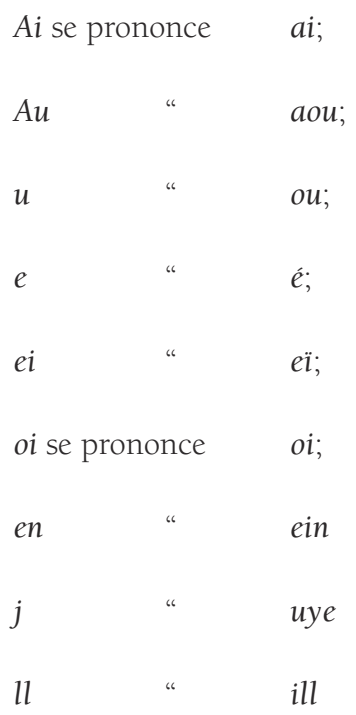


98

$\tilde{n} \quad$ " $\quad$ gn

$s$ se prononce $\quad s g$

$t t$, tt mouillé appuyer le milieu de la langue contre le palais

$z$ se prononce $\quad c e$

g est toujours dur en basque.

\section{oOo}

Tantugou duçu gizon zahar bat oihanetan gordatcen dena. Zaintzentzi landac eta phentziac ez dezaten deus ebats. Ogiac eta belharrac phicatiac dienian, nausiac oro barneat bildu artio, Tantugouc ez dici loric iten: bethi beilan dagozu. Haren bichtarat agertziaren beldurrac beiratzentzi ohoinac. Hunauzu, seur, bena basauzu. Hanichco gauza zakien laborari batec erraiten zizin ardura bere semer Tantugou zela seurki lurreco frutien zaintzaleik hoberena.

Uztailaco arratsalde batez, arthalde bat alha zuzun mendi baten gainian: artzaina lokhartu zuzun; iatzartziarekin ohartu zuzun Tantugou laster zuala oihaneco aldeat. Guisa berian ezagutia izantzuzun behi zaintzalez ordoki poillit batian etzana harroca baten gainian.

Orai oono fonteraco (Pyrénées) zombait herritan, haurrac haren beldurtzu. Muthico ttipi edo nechcato ttipi batec ez dienian bere ama obeitu nahi, hunec erraiten dacozu: "Guarda emac, ou kasu emac (eman, pour la fille) oihu egiten dacoat (saconat pour la fille); Tantugouri eta, ichtantian, jin duc ( $\underline{\text { dun }}$, pour la fille) oihanetic.

Miguras, 39 ans, Ahetze Coll.: Barran, Delgue, Iriart 


\section{Barbazaneco erran zaharra}

Demboran Pyreneco herrichca batian bici ciren guizon eta emazte zahar zahar batzu. Ez zuten etche ttipiño bat baratze bat eta behi bat baicic. Bizkitartean hain beharrac izana gatic, bazakiten berac baino beharragoen laguntzen. Egun batez lurra elhurrez estalia cen, harri arrailtzeco horma ari zen. Herrico yende guciac yaten eta edaten ari cirelaric su phazterrean berotzen zauden aberatsac eta urusac ciren. Astirian, nonbait urrundic heldu ciren bi piayantec hotzac eta gosiac zirelacotz nahi izan zuten herri hartan gelditu, yoten dute bortha bat, guero berce bat, eta oraino berce asco. Nihorc ez cituzten sartcerat uzten ez deus yaterat emaiten. Gucietaric casatuac, chakhur borthitz batzu ehausiz ondotic, ez zakiten norat yuan bi arrotzec, eta helduac ciren herriaren azken bururaino, guizon eta emazte behar haukien etche aitzineraino. Haukien intziriac entzutearekin senharra aguertu cen leihora haien sararazteco eta emaztea laster yuan zen bortharen idekitzerat. Galdeguin gabe nor sien, sar-arazten dituzte, eta su ondoan yar-arazten: guero cerbitzatzen diote esnea, gaztainac, eta ciusten gucietaric. Orduan Yesus-Christo gure yauna -ecen hura cen yon doni Petrirekin- chutitu cen burua argui distirant batez inguratua eta eraiten diote: "Zuec beharrac eta onac ciezte, aldiz zuen hauzoac aberaxac eta zarrac Yaincoaren yusticia eguin dadiela!". Hainbercenarekin lurra ikharatcen da. Yesus eta Yon done Petri eceztatzen eta bi zaharrac belhaunico erortzen dire. Biharamun goizean yuan cirenean herrirat yakiteco cer iragan cen. Ez zuten etche bihi bat ikhusi bainan heien orde aintcira handi bat (ur beltz handi bat) egun oraino Barbazaneco herriaren eta San Bertran Comminyeco hiriaren artian ikhusten dena. Dohacabeac behar dire bethi lagundu.

\section{Tantugouren gainean erran zahar bat}

Tantugou oihanetan gordetzen den guizon zahar bat da. Landac eta phentzeak beguiratzen ditu ez dezaten deus ebats. Oguiac eta belharrac picatzen direnian, nausiac gucia sarthu arteraino Tantugouc ez du loric eguiten: bethi iatzarria dago. Beldurrez hura aguer, ohoinac urrun daude. Ona da, sinhets zazue, bainan basa. Lur lantzale hainitz gauza zakien batec erraiten zuen bere semeeri Tantugou cela segurki lurreco fruituen beguira zaileric hoberena.

Uztaileco arratsalde batez arthalde bat alhan zagon mendi batean, artzaina lokhartu cen. Yatzarcearekin ikhusi zuen Tantugou oihanerat ihesi espacatzen. Behi zainzalec oro bat ezagutu zuten celaya pollit batean, harroca baten gainean etzana.

Egungo egunean oraino, Pyreneco zembait herrietan haurrac haren beldur dira. Muthico edo nechcato batec ez dionean amari onhexi nahi "Guarda eman, erraiten dio amac, Tantugou-ri oihu eguiten dinat eta berehala oihanetic yinen dun

Chimits Pierre, 47 ans, né à Chéraute (B. Pyrénées) Coll.: Etchevers, maire, Etchebéhère (adjoint) 


\section{Barbazanen gaignian erran çaharra}

Baçouçoun lehen Pyrénéetaco herri batian guiçon bat eta emazte bat çoinac biciqui çaharrac baitciren. Ez cicien etche tipi bat, baratce bat eta behi bat becic. Béna hain pobre içana gatic, lagountcen citcien bethi hec beno pobrio cienac. Egoun batez, lourra coucoutia çouçoun elhourrez, eta kharroin iten cicin harria arrailtcerainocouan. Herrico yende gouciac berotcen ari citçoun, yatiaiquin eta edatiaiquin; aberats eta irous citçoun. Ilhoun nabarrian bi bidayant, çoinac heldou baitcien ourrounic, nahi oukhan cicien baratou lekhou hartan, ceren eta gose eta hotz baitciren; yo cicien bortha batian, guero beste batian eta beste batçoutan oono: nehoc e ez citcin sartcea outci nahi oukhan, ez eta deous ere heer yatea eman. Casatiac orotaric, seguitiac chakhour fuchosez, bi estranyerrec ez ciakicien norat youan, noiz eta ere khaousitu baitciren herriaren bazterrian, pobre cien emazte eta guizon heen etchiaren aitcinian. Intçoutiaiquin heen plaintak, senharra yarri çouçoun leihouan heer oihou iteco, eta hain emaztia jouan çouçoun fite borthain idokitcea. Batere heer galdin gabe nor cien, sarraazi citcien eta yararaci beren soupazterrian; guero cerbitchatou ciecien esnia eta cembait gaztaïgna, ciouzten gouciac. Bena ordian Goure Yainco Yauna -houra çouçoun Yan Doni Petrirekin - yeiki çouçoun buria arras arguitan, eta erran cieçoun; "Ciec pobriac eta hounac ciezte; cien aouçouac aberatsac eta gaichtouac tçou. Yaincoaren yousticia in dailela!". Behala lurra ikharatou çouçoun, Yesous eta Yan doni Petri bichtatic galdou citçounn eta bi çaharrac erori citçoun belhaounico... Biharamoun goician, noiz eta ere youan nahi çoutenian herrira yakiteco cer phasatou cen, ez cicien ikhousi etche bagnore: heen plaçan, baçouçoun phoutçou handi bat çoina baita oono egoun Barbazaneco herriaren eta Saint-Bertrand-de Commingeco hiriaren artian. Behar tcie lagoundou malourosec.

\section{Tantugouren gaignian erran çaharra}

Tantugou douçou çahar bat çoina gordatcen baita oyhanetan. Beiratcen tci alhorrac eta phentciac han ez deçaten ebats deous ere. Noiz eta ere oguiac phicatiac dienian edo belharra ebakia, oro erretiratou artio naousiaz, Tantugou ez tçou lokhartcen; beillatcen dici bethi. Haren aguertciaren ikhousteco beldourrac atchikitcen tci ohoignac. Houna douçou, sinhets çacie, bena salbaya douçou. Laborari batec, çoinac baitçakien anhichco gaouça, erraiten cicin ardoura bere semer Tantugou cela seourki lourreco frouitien beiraçalerik hoberena.

Ouztaillaco hilabeteco eouguerdi ondo batez, ardi tropa bat alhatcen çouçoun mendi baten gaignian; artçaigna lokhartou çouçoun: iratçartciaikin apercebitu cicin Tantugou, çoinac ihes iten baitcien oyhaneco alderat. Içan çouçoun berdin eçagoutia cabalaçaignez, ordoki poillit batian, etçana arroca baten gaignian.

Oono egoun, Pyrénéetaco cembat herritan, haourrac beldour tçou hain daco. Noiz eta mouthico tipi batec edo nechcato tipi batec e dienian obeditou nahi bere amari, hounec erraiten diacoçou: "Khasou in tçac hiaouri! deitcen diat Tantougou, eta, ichtantian, yinen douc oyhanetic"!

Etchart, 42 ans, Jaxu Coll.: Arnaud Etcheberry 
Baziren bestorduz Espagnaco funteraco mendier contraco herri batian gizon bat eta emazte bat çahartiac. Etzuten etche ttipi bat baratce bat eta behi bat becic. Bainan praubiac itzanik ere, sokhorritzen ziuzten bethi hec beino beharraguac zirenac. Egun batez lurra estalia zen elhurrez eta kharroin bat gaitza ari zen. Herrico yende guzia berotzen zagon untsa yaten eta edaten zielaïc; aberats eta irus zien. Ilhuntcian, bi bidayantec, heldu zienac urrunetic nahi ukhan zuten baratu lekhu hartan, zeren hoztiac eta gossetiac beizien; yoiten dute bortha batian, guero beste batian eta beste barnetan ere, nehorc eziuzten nahi ukhan utci sartcerat ez eta ere deussic eman yatera. Kassatiac orotaic, chakhurrez akhulatiac, bi estranyerrec etçakiten nora yuan noiz eta ere khaussitu baitziren herriaen bazterrian, erran den guizon eta emazte praube heyen etchearen ainzinian. Inzutiarekin heyen plegniac, senharra yuaiten da leihora, deithacen tu eta haren emaztia yuantzen fite bortharen zabalcera. Galdeguin gabe nor ziren, sarrasten tu eta yarrazten supazterrian, guero emaiten de esne eta zembeit gaztagna, hec ziuztenac oro. Bainan orduan Salbazaile yauna: cen hura zen Yondoni Petrikin, chutizen da, buria guzia arguiz distiratzen zuelaric eta erten dee: "Ziec, praube eta hunac ziezte; zien auzoac, aberats eta gaichtoac dira. Yaunaren justicia eguin dadila!". Berehala lurra ikharatcen da, Yessus eta Yondoni Petri bichtatic galzen dira eta bi çaharrac yartzen dira belhaunico. Biharamun goitzian, yuan nahi ukhen zutenian herrirat, yakiteko zer phassatuic cen, etzuten ikhussi etcheric batere; heen plaçan phutzu handi bat zen, zoina khaussitzen beita oraino égun, Barbazan deitzen den herriaren eta San Bertran de Cominge deitzen den hiriaren artian. Behar dira lagundu malerussac.

\section{oOo}

Tantugou çahar bat da gordatzen dena oihanetan. Zaintzen tu alhorrac eta phenciac nehorc deus ebatsi gabe. Oguiac phikatzen direnian edo belharrac ebakiac, naussiac oro bildu artino Tantugouc ez du batere loic eguiten, bethi zain dago. Bethi noiz aguertuco den beldurraz, daude ohoinac. Huna da, sinhes zazie, baina salbaya da. Laborari yakintsun batec erten zuen ardura bere semer Tantugou zela segur lurreco fruituen zaintçaleic hoberena.

Uztaillaco axalde batez ardi tropa bat phassatzen zen mendi baten gainian; arçagna lokharzen da; irazarriakin ohartcen da Tantugou lasterca duala oihanari buruz. Içan zen berdin eçagutia arçaignez mendi zola batian, etçana arroca baten gainian.

Oraino egun bortu ondoco herri batzutan, haurrac beldur dira harendaco. Muthico ttipi batec edo nechkato batec ez dienian obeditu nahi amari, amac erten dee: Guardaman! oihu eguinen dakonat Tantugouri eta berehala jinen dun oihanetic 


\section{Barbazeneco ichtoria}

Bazen lehenago Pirenetaco herri batian guizon bat eta emazte bat biziki zahar zienac. Etzuten etche ttipigno bat, baratze bat eta behi bat bezic. Bena hain probiac izanaatic sokhorritzen zituzten bethi berac beno biziki probiaguac zienac. Egun batez lurra elhurrez kukutia zen, eta kharrougn bat eguiten cien harriaren erdiatzekua. Herrico yende guciac berotzen ari zien, yatiaikin eta edatiaikin; aberax eta irus zien. Ilhun naarrian, bi bidayant urrunic heldu zienac nahi ukhan zuten lekhu hortan baratu, zeren hotz eta gose bitzien; yo zuten bortha batian, guero beste batian eta oono beste azutan: nehokee etzituzten sartzea utzi nahi ez yatea yeus eman nahi. Kasatiac orotarik, chakhur borthitzez persekeitiac, bi estranyerrec etzakiten norat yuan, noiz eta ere khausitu bitzien herri bazterrian guizon eta emazte probe zien hoien etchiaren aitzinian. Intzutiaikin heen plegniac, senharra emantzen leihuan, heen deitatceco eta emaztia yuantzen behala borthain zaaltzea. Galdiin gabe nortzien, sarraazi eta yarrazi zuzten suphazterrian; guero eman zezteen ziuzten esne eta gaztagna guziac. Bena ordian, Gure-Salbazailia, -huazen Yondoni Petriikin, - chutitu zen, buria argui arguia, eta errantzeen: "Ciec, probe ziezte eta hun; zien auzuac aberax dira eta gaichto. Yinkuain yustizia ina izan dadila!". Ordu berian lurra ikharatu zen, Yesus eta Yonboni Petri ezeztatu zien eta bi zaharrac erori zien belhaunico... Bihamoun goizian, nahi ukhan zutenian yuan herrirat, yakiteco ze phasatu zen, etzuten ikhusi etche bihi bat: heen lekhian phutzu handi batzen, egun oono khausitzen dena Barbazaneco herriain eta San-Bertrand-de-Commingeco hiriain artian.

Sokhorritu behar dira miserabliac.

\section{Tantugouin ichtoria}

Tantugou oihanetan gordatzen den guizon zahar bat da. Beiratzen tu alhorrac eta phentziac amoreagatic eta hetan yeus ez dezaten ebax. Noiz eta ere oguiac pikhatiac beitira edo belharra ebakia, agnic eta nausiac oro sarthou artio, Tantugouc ez du loik batere eguiten; bethi guardian doo. Haren aguertzen ikhustearen beldurrac atchikitzen ditu ohognac. Huna da, sinhexazie, bena basa da. Biziki gauza zakien laborari batec erreiten zien ardura bere semer Tantugou zela segurki lurreco fruitien zaintzaleik hobeena. Uztailla hilaiteco axalde batez, arthalde bat alhantzoon mendi bizkar batian; artzagna lokhartu zen: iratzartziakin ohartu zen Tantugou ihesi zuala oihaneco aldeat. Orobat ezagutia izantzen artzagnez mendi arte pollit batian, harroca baten gagnian etzana. Egun oraino, Pirenetaco zumbeit herritan, haurrac beldur dira haindaco. Noiz eta ere muthico ttipi edo nechkato ttipi batec ez beitu nahi bere amari obeditu erraiten daco: Guardimac hiri! Oihu iten dakoat Tantugouri eta, mementian, yinen duc oihanetic

Etchégoinberry, 21 ans, né à Beyrie-sur-Joyeuse

Coll.: Lespade et Bidegain Pierre 
Bacen leheno, Pyrenetaco herri batian, guiçon bat eta emazte at biciki chahar cienac. Etçuten etche ttipi bat becic, baatce at eta behi bat. Bena combat nahi probe cien sokhoritcen çusten berac beno probio cienac. Egun batez, lurra koukoutia cen elhurrez eta kharrougn iten cien harrien zartasteco guisan. Herrico yende guciac, beotcen cien, yatiaikin eta edatiaikin; abeax cien eta irous. Ilhountcian, bi bidayant, ourrounic heldu cienac, nahi ukhan çuten baatu lekhu hartan hotz eta gose baitcien; yo çuten bortha batian, gueo beste atian eta beste batçutan oono; nehokec ere etçuzten utci nahi ukhan sartcera, ez eta ere eman deuse yatea. Casatiac gucitaic, lastercatiac chakhur coleratu batçuz, bi estranyerrec etçakiten noat yoan, noiz eta ere khausitu baitcien herri basterrian, probe cien guiçon eta emazte hen etche aitcinian. Intçutiaikin hen plegniac, senharra yarri cen leihouan her oihu iteco eta hain emaztia yuan cen fite borthain çaaltcea. Galdin gabe nor cien, saraci çuzten eta yarraci su pasterrian. Gueo cherbitchatu ceeten esne eta çombait gastagna, çuzten guciac. Bena ordian gure Yncua, - hua cen Yondoni Petriikin - chutitu cen, buria argui arguia, eta erran ceen: 'Ciec probe eta houn cizte, cien ausuac abeaxac eta gachtuac dira. Yncoain yustician in daila". Dembora berian, lurra ikhaatu cen, Yesus eta Yondone Petri galdu cien eta bi chaharrac belhaunicatu cien. Bihamun goician, nahi ukhan çutenian yuan herrira, yakiteco cer pasatu cen, etçuten ikhousi etcheate; hen lekhian bacen phutçu bat oono hatcemaiten dutena Barbazaneco herriain eta Saint Bertrand de Comingesco hirriain artian.

Behar dira probiac laundu.

oOo

Tantugou chahar bat da gordatcen dena oihanetan. Beguiatcentci alhorrac eta phintciac ebax ez deçaten deuse. Oguiac phicatuic dienian edo belharra ebakiic, gucia bildu artio nausiac, Tantugouc ez du bate loic iten; iratçarriik da bethi. Haren aguer beldurrac gueldic egonasten tu ohognac. Houna da sinhex cacie, bena salbaya da. Laborari batec, hanitz gauça çakienac, erten cien usu bere semer Tantugou cela segurki lurreco fruten beguirariic hobena. Ustailla axalde batez, ardi saldo bat baskatzen cen mendi batian; artçagna loc hartu cen; iatçarciaïkin, aprecebitu cien Tantugu espacatcen oïhaneat. Hala hala eçautia içan cen behi çain batçuz ordoki poillit batian, etçanic harrobia batian. Egun oono, Pyrenetaco herri çonbaitetan, haurrac haen beldur dia. Mothico ttipi batec edo nechcato ttipi batec nahi ez dutenian obeditu bere amari, erten daco: "Abisa hadi; oïhu iten diat Tantuguri, eta ichtantian ynen duc oïhanetic.

Jauréguy Pierre, né en 1858 à Musculdy Coll.: Jean Etchemendy et Aldacour Guillaume 
Bacen lehenogo, Pyreneetaco herri batean guiçon bat eta emaste bat arraz çahartuac. Ez çuten etche ttipi bat baicen, baratze bat eta behi bat. Bainan, hain pobreac içana gatic ere, socoritcen çuten bethi hec baino pobreago cirenac. Egun batez, lura elhurrez estalia cen, eta harien erdirasteco karoïna eguiten cuen. Herrico jende guciac berotcen ciren, jaten eta edaten çutelaic; aberats ciren eta uros. Ilhuntcian, bi bideyent hurundic heldu cirenac nahi içan ciren guelditu toqui hartan, ceren hostuac eta gosetuac baitciren; jo çuten bortha bati, guero beste bati, eta aintcina beste batçuei: nehorc ez ciusten nahi içan utci sartcerat, ez deusic eman yatera. Casatiac gucietaric, perseguitiac chacur gaitcez, bi estrangerec ez çaquiten norat juan, noiz eta khausitu baitciren heriaren basterian, guiçon eta emaste pobre heien etche aintcinean. Entçutiarequin heyen pleiniac, senhara leihoratu cen heyen deithatcera, eta haren emastiac laster eguin cuen bortharen çabaltcerat. Galdeguin gabe nor ciren, sarraraci ciusten eta jarraraci su phasterrean; guero, cerbitchatu cioten ezne eta cembait gastana, ciusten guciac. Bainan ordian, Jincoa -houra cen Jondoni Petriequin,- jeyqui cen, dena distiranta eta erran cioten: "Ciec, pobreac ciste eta onac; çuen ausuac aberatxac dire eta gaichtoac. Jincoaren justicia eguin dadiala!". Ordu berean, lura ikharratu cen, Yesus eta Yondoni Petri galdu ciren, eta bi çaharrac erori cien belhaunica. Bihamun goicean, juan cienian herirat, jaquitera cer iragan cen, ezçuten ikhusi etcheric batere; hequien plaçan, bacen laco bat, oraino egungo egunean khausitcen dena Barbazaneco heriaen eta San-Bertrand-Commingeaco hiraren artean. Behar da lagundu malerusac.

\section{oOo}

Tantugou oihanetan gordetcen den çahar bat da. Saintcen tu alhorrac eta phentxiac deusic ebatz ez deçaten. Oguiac edo belharra ebaquitiac dienian, denac nausiac sar artino, Tantugouc ez du loric eguiten; bethi guardian dago. Haren ikhus beldurrac atchiquitcen tu ohoinac. Ona da, sinhets açué, bainan salbaya da. Laborari, hainitz gauça çaquien batec erten cien ardura segur Tantugou lureco fruituen guardinoric hobena cela.

Ustarilaco arratxalde batec, ardi saldo bat alha çagon mendi batian; artçaina loac hartuc cien; irratçarciarequin, ikhusi cien Tantugou espacatcen oihaneco alderat. Içan cen oraino eçagutia artçainez vaillea pullit batean, etçana arroca batian.

Oraino egungo egunean, Pyreneetaco cembait herritan, haurrac harendaco beldur dira. Muthico edo nechcato ttipi batec ez dienian sinhetsi nahi bere amari, erten dio: Guardia emac hiri! Jinaracico diat Tantugou, eta ichtantian, jinen duc oihanetic! 


\section{Barbazaneco ichtorioa}

Bazen lehenago Pyrénéetaco herri batian guizon bat eta emaste bat arras zahar cirenac. Ez zuten etche ttipi bat, baratce bat eta behi bat baizic. Bainan zombat nahi pobre ciren, socorritcen ciuzten bethi hec baino pobriago cirenac. Egun batez, lurra elhurrez coucoutia cen eta kharroina ari cen harriac arrailtcerainocoan. Herrico yende guciac berotcen zauden, yaten eta edaten ari cielaric; aberats ciren eta uros. Ilhun nabarrian, bi piayant urrunic heldu cirenac, nahi izan ciren guelditu lekhu hartan, ceren hotçac eta gosiac baitciren: yo zuten bortha bat, guero beste bat eta bertce batçu oraino: nehorc ere ez ciuzten utci nahi sartcera, ez eta ere deus eman yatera. Orotaric casatiac, chakhur fuchos batçuez seguitiac, bi estranyerrec ez zakiten norat yuan, herriaren bazterrera cirenian guizon eta emazte pobre hen bortha aitcinian. Heyen plegniak entçutiarequin, senharra leihuan eman cen heyer oïhu eguiteco eta emaztia yuan cen fite bortharen zabaltcera. Galdeguin gabe nor ciren sarraraci eta yarraraci ziuzten beren supasterrian; guero cerbitçatu zeeten esnia eta çombait gaztena, ziuztenac oro. Bainan ordian, Jesus-Christo - hura cen, Yondoni Petrirequin- yeikitcen da, buria oro distirant, eta erraiten dere: "Ciec, pobre eta hun ciezte; cien aüzuac aberats eta gaichtuac dire. Yaincuaren yusticia eguina izan bedi!". Berheala lurra ikharatu cen, Jesus eta Yondoni Petri ezeztatu ciren eta bi zaharrac belhaunico erori... Biharamun goician, nahi izan cirenian herrirat yuan yakitera cer passatu cen, ez zuten ikhusi etcheric batere: heyen lekhian ur handi gueldi bat bazen, egun oraino causitcen dena Barbazaneco herriaren eta San-Bertrand- de- Commingeco hiriaren artian.

Malhurosac lagundu behar dire.

\section{Tantugouren ichtorioa}

Tantugou guizon zahar bat da oihanetan gordatcen dena. Beguiratcen tu alhorrac eta phentciak deus ebats ahal ez dezaten. Oguiac picaturic direnian eta belharrac ebakiric, nausiac bildu artino, Tantugou ez du loric eguiten; bethi iratçarria dago. Haren ikhusteco beldurrac dauzca ohoïnac. Ona da, sinhets zazu, bainan basa da. Laborari batec hanits gauza zakienac erraiten zien ardura bere semer, Tantugou cela segurki, lurreco fruituen beguiraric hobena.

Uztaileco ilhabetian eguerdi ondo batez, ardi tropa bat alha cen mendi batian: artçaina louac hartu cien; iratçartciarekin, ikhusi cien Tantugou oïhanerat ihesari emaiten. Orobat ezagutia izan cen artçain batçuez baillea poillit batian, etçana harroca baten gainean.

Egun oraino Pyrénéetaco zombait herritan, haurrac haren beldur dira. Muthico ttipi batec edo nechcato ttipi batec ez dienian obeditu nahi bere amari hunec erraiten daco: Guarde emac (edo eman)! Tantugouri oïhu eguinen diat (edo dionat) eta berheala yinen duc (edo dun) oïhanetic!

Duhalde, 46 ans, né à Suhescun. Coll.: Etchepareborde, ancien maire, âgé de 72 ans 
Baçuçun lehenago, Pyrénéetaco herri batetan; guiçon bat eta emaste bat, hanitz çahar cirénac. Etcicien etche tchipi bat, baratce bat eta behi baicic. Bena, çoumbat nahi praübe cien, berac béno praubiago cirénac, bethi socorritcen citicien. Egun batez, lurra elhurrez gorderic çuçun, eta icigarrico kharroua ari çuçun. Herrico jentiac oro, berotcen ari çutuçun, jaten eta edaten cielaic, abératx eta irous çutuçun. Ulhuntcin, bi bidayant, hurruntic jiten cirénac, nahi içan çutuçun ukhuatu lekhu hartan, ceren hotz eta gosse beitciren. Jo cicien bortha bat, guero beste bat, eta guero bestéic orano; ihourkere etciticien nahi ukhen utci sartcéra, ez deüs éré eman jatéra. Orotaric cassaturic, hor khéchatiéz persébituric, bi bidajentec etçakicien nouat joun nouiz eta éré edireiten beitira herriaren basterrian, guiçon eta emaste praübe hayen etché aitcinian. Hayen plaintac entçutez, senharra elkhi çuçun leïhoula, hayen deitceco, eta emastia joun çuçun bertan bortharen çabalcéa. Galthatu gabé nour ciren, sarréraci citicien eta jarréraci beren suthondun; guéro, cerbutchatu cieçun esne eta gastagna çoumbait, ciéna oro. Bena ordin, Goure Jinco Jauna - houra çuçun, Joundane Phétiriékin- jeiki çuçun, buria argui arguia, eta erran cieçun: "Ciec, praübe et houn cirié; cien aiç̧ouac abératx eta gaisto dutuçu. Jincouaren justicia eguin bedi!". Hambesténarekin, lurra ikharatu çuçun eta bi çaharrac erori belhaïco... Bihâmun goiçan, nahi içan cienin joun herrila, jakiteco cer igaran cen, etchéric batére etcicien ikhusi: heyen lekhin, baçuçun, losco bat, orano orai éré edireiten déna San Bertrand de Commingeco hiriaën eta Barbazaneco herriaen artian. Malerousec behar dicié socorritu.

\section{oOo}

Tartaroua duçu guiçon çahar bat, oyhanétan gordatcen dena. Alhorrac eta sorhouac beguiratcen ditiçu, deüsere ebatx estecen. Oguiac eta belharra ebakiric direnian, buruçaguiac oro bilartio, Tartaroua estuçu lotcen; bethi beillatcen duçu. Haren aguertcen ikhusteco lotxac beguiratcen ditiçu ouhougnac. Houna duçu, sinhex eçaçu bena bassa duçu. Hanitz gaiça çakien laborari batec erraiten çiçun ardura bere semer lurreco fruten beguiraçaleric hobena cela Tartaroua.

Ustarila arrestiri batez, ardi saldo bat alha çuçun mendi batetan; artçagna loac hartu ciçun; iratçartcian abisatcen duçu Tartaroua baçouela ihes, oïhanilat buruz.

Ulhain eli batec ere eçagutu cicién naba eger batetan, arroca baten gagnen, etçanic.

Egun ere orano, Pyrénéétaco herri çoumbaitetan, haürrac haren lotxa dutuçu. Muthico tchipi batec eta nechcatila tchipi batec amari estenian nahi obéditu, amac erraiten dioçu: Abisa hadi! Deitcen diat Tartaroua eta behala jinen duc oyhanetic. 
Bazen lehenago Pyrénetaco herri batetan gizon bat eta emaste bat hanitz zahar zienac. Etzien etche chipi bat, baratze bat eta behi bat baisic. Bena zounbat nahi praübe isanic ere, sokhorritzen zutien bethi berac beno praübigouak. Egun batez lurra gorderic zen elhurrez eta kharrouak erdiatzen zin. Herriko jentic oro berotzen ari siren jatiarekin eta edatiarekin, aberatx eta irus ziren. Ulhuntzin, hurruntic jiten zien bi bidayant nahi izan zien arreztatu lekhu hartan seren gose eta egarri beitzien, jo zien bortha batetan, gero beste bat batetara eta orano beste bat sounbaitetan; ihourc ere etzutien nahi ukhen utzi sartzera es eta deuse eman jatea. Ohilturic bazter orotaric, jarraikiric hor malesian zien elibatez, bi arrotzek etzakien nourat jouan, nouiz eta ediren beitziren herriaren bazterin guizon eta emazte praübe harien etchiaren aitzinin. Haien plagnien entzutiari, senharra jarri sen lehoun hen deitzeko eta emastia jouan sen bertan bortharen sabaltsera. Her galthatu gabetaric nour ziren, sar errassi zutien eta jar errassi beren suthondoun, gero zerbutchatu seyen ezne eta gastigna sounbait, sutienac oro. Bena, ordin, Goure Jaüna, - houra cen Jon Joundane Phetirirekin jeiki sen buria argi argia eta erran ceren: "Ziec praube eta houn zidie, zein aizouk aberatx eta gaisto dira: Jincouaren justicia egin dadila!". Hain sari lurra ikharatu sen, Jesus eta Joundane Phetiri galdu siren eta bi saharrak erori siren belhaiko... Biharamen goizan, joun nahi izan sirenian herrila jakiteko ser igaran sen, etzien ikhoussi etcheric batere; haien lekhin basen losko handi bat souin ere edireiten beyta orano egun Barbazaneko herriaren eta Saint-Bertrand-de-Comminjeko hiriaren artin. Behar dira laguntu malerusak

\section{oOo}

Tantugou gizon zahar bat oihanetan gordatzen dena. Begiatzen tu alhorak eta sorhouak, amourekati han deuzere ebax estesen. Nouiz eta ogiak mousturik beitira edo belharrak ebakiric, oro buruzagiz sarthurik izan artio, Tantugou ezta batere lotzen, iratzarririk dago bethi. Haren ikhousteco loxak baratzen tu ouhouñak. Houn da sinhex ezazu, bena salbaje da. Gaiça hanitz sakin laborari batek erraiten zien usu bere semer, Tantugou zela segur lurreko uzten begiariric hobena.

Uztailaco arraztiri batez ardi-saldo bat alhan ari zen mindi baten gañen; artzaña lothu zen: iratzarsiareki ikhousi sin Tantugou oihanilat eskapatzen. Ezaguturik izan tzen ere behi begirazalez, arbailla ejer batetan, arroka baten gañen etzanik.

Orano egun Pyreneetaco herri zoumbaitetan haürrac harren loxa dira. Nouiz ere mothiko chipi batek edo neskachou batek ezpeitu nahi obeditu bere ama hounec erraiten deyo: Kasu egin esak hiri! deitezen dut Tantugou eta hain sari jinen da oihanetik

Laplume, âgé de 45 ans, né à Moncayolle Coll.: M. Daguerre; M. Sagardoyburu 


\section{Barbazaneco errankiçuna}

Baçutuçun bestordus Pyrénéac deitcen dien borthien khantuco herri chipibatetan senhar emasté élibat hanitch çaharrak. Praoué çutuçun; etcicien etché chipi bat, baatcé bat eta behi bat baïcic, bena, çoumat nahi praoué içan citin, bethi beak beno praué handiagouk sokhorritcen citicien. Egun batez lurra elhurrez gorderik çuçun eta kharroua aï haïn borthiski noun udui beïtcin harrik é erdiatu behar çutila. Herri chipi hartaco gentik oro been etchetan beotcen aï çutuçun, jaten eta edaten cielaïk, abéatz eta ious beitcien. Ulhuntcin, bi bidejant çougn hurrunti horra beïtcien, ekhuatu nahiçan çutuçun herri hartan, ceen gosia eta hotça sendi beitcien. Jo cicien bortha bat eta beste hanitch oano: ihouné etciticien utci nahiçan sartera ez eta eman jatea. Ootaïk kassatuik, cakhur borthitz élibatez jarraïkirk, bi estrangé hec etçakicien nouat joun nouiz eta herriaen basterin aguitu beïtcien guiçon eta emasté hayen etché aitcinin. Bidejanten plaïntak entçun beçagn sarri, naousia joun çuçun leïhoula hen deïtcea eta ber denboan emastia borthain sabaltcea hen barneat sarrasteco, galthegin gabé nour cien. Been suthondoun jarraci citicien eta eman ezné eta gastagna ezpeïtcien deuzé besteik. Bena ordin, Jesus Christ, ceen houa beïtcen sen Pierraeki, jeïki çuçun, alagueatuik eta senhar emasté her erran cieçun, "Ciec praué eta houn cidé, cien aïçouk aldiz abeatz eta gaïchto: Jincouaen justicia eguinik içan dadila!". Ber denboran lurra ikhaatu çuçun, Jesus-Christ eta sen Pierra hanti galdu eta senhar emasté çahar houk belhaïko jarri. Bihamenin, nouiz eta senhar emaste praoué houk herrilat joun nahiçan beïtcien jakitea cer igan cen, etcicien etcheik ikhousi. Hen lekhin oro hour handi batec hartucik çuçun eta egunco egunin oano hour houa edieïten duçu Barbazaneco herriaen eta Sen Betran-de-Commingues deïtcen den hiaen artin. behar diela.

Feit horrec markatcen eta probatcen dikuçu maleous dienac laguntu eta sokhorritu

\section{Tentugouen errankiçuna}

Tentugou duçu oïhanetan bici den guiçon çahar bat. Ber demboan maité diçu besten alhor eta sohouen beguiatcia, gaïski eguilen ephantchatceko deuzé ebasteti. Nouiz eta oguik phikatuik eta belharrak ebakik beïtira; houk naousik barneat bil artino, Tentugou eztiçu loik eguiten, gaï eta egun beillan duçu. Haen ikhousteco lotsak ouhougnak ephantchatcen tiçu gaïski eguiteti. Guiçon houna duçu, bena salbaje duçu. Gaïça hanitch çakin laboaï batek bé semer usu erraiten cieçun Tentugou cela segurki lurreco fruten beguiraïk hobena.

Ousthaïla deitcen den hilabeteco arristi batez ardi saldo bat bascatcen aï çuçun mendibatetan, hen artçagna louak hartu ciçun eta iatçartciaëki apercebitu ciçun Tentugou oïhanilat eskapatcen cela. Besté artçagn élibatec é eçagutu cicien naba éjer batetan etçanic celaik arroka batetan.

Egun oano Piréré deïtcen dien bortu ondoko herri çoumbaïtetan, Tantugou deitcen den guiçon haen haourrak loxa tuçu: Nouiz eta é haourrek mothiko edo neskatila ican ditin, espeituke been amak sinhetsi nahi, hounek erraiten dieçu: "Abisa cité deïthuko dut Tentugou eta behala jinen da oïhaneti."

Elhe horrec haourrac obédient errendatcen tiçu.

P. P. Salles, 27 ans, né à Garindein (Bsses Pyrénées) Coll.: Garat, Pierre 


\section{Barbazan-en ichtoria}

Beste aldiz bort'ondoko herri batetan, bazutuzun gizoun bat eta emazte bat izigarri zahar zirenak. Etzizien etche tchipi bat, baratze bat eta behi bat baizik bena zoumbat nahi praube ziren, sokhorritzen zitizien bethi berak beno praubigouk. - Egun batez elhurrez lurra gorderik zuzun, eta ber denboran ari zuzun kharrou izigarri bat. - Herri hartako jentik oro, jatez eta edatez, zagotzun beren suthondoutan: aberats eta irous beitzien. - Ulhuntzin, bi bidajant, hurruntik jiten zienak, ukhuratu nahi izan zien lekhu hotan, gose eta egarri zielakotz; jo zizien bortha bat, gero beste bat, gero besterik hanitch, ihourk ere ezitititzun sartzera utzi ez eta deus eman jatera. - Orotarik igorririk, hor gaitz elibatez lasterkaturik, bi estranjerek etzakizien nourat joun: nouiz herri bazterrila ondoun, gizoun eta emaste praoube haien etche aitzinin agitzen beitira. Hen oihik entzunik, senharra leihoulatzen duzu hen deitzeko eta emaztik bertan bortha zabaltzen duzu.

Arainkuratu gabe nour zien, sar erazi zitizien eta jar erazi bere suthondoun; gero zerbutchatu ziezun ezne eta gaztaña zoumbait: etzizien besteik.

Bena ordin, goure Jaouna, - ezi houra eta bera zuzun Joundane Phetirireki, - jeiki zuzun, argiz inguraturik eta erran ziezun: "Zuk, praube izana gatic, jente hounak zidie, zien aizouk aberats eta gaichto tuzu; - Jinkouren justizia eginik izan dadila!"

Hainbestereki, lurra ikharatu zuzun, Jesus eta Joundane Phetiri galdu zutuzun eta bi zaharrak belhaiko erori.

Bihamen goizan, nahi izan zienin joun herrilat, jakitera zer agitu zen, etcherik etzuzun haboro ikhousten, hen lekhin, hour handi bat (lac) egun orano edireiten dena Barbazzeneko herriren eta Saint Bertrand de Comminges deitzen den hiriren artinBethi beharduner lagungoua eman dezegun.

\section{Tantugou-en ichtoria}

Tantugou oihanetan gordatzen den gizoun chahar bat duzu. Begiratzen tizu alhorrak eta sohouk, han deus ebats eztadin. Nouiz ere ogik mouzturik edo belharrak ebakirik beitira, oro buruzagik etcherat bil artino, Tantugou eztuzu lotzen, bethi gouitan duzu. Haren lotsak etchekiten tizu ouhouñak. Houn duzu zegur, bena gaitz eta moulde gabe. Hanitch gaiza zakin laborari batek usu bere semier erraiten zie/ozun Tantugou zela segur lurreko hountarzunen begirari hobena.

Uztailako arristiri batez, ardi saldo bat alha azi zuzun mendi baten gañen: artzaiña lothu zuzun. Iratzartzin ikhoussi zizun Tantugou ihes egiten oihanilat. Bardin ezaguturik izan zuzun artzaiñ elibatez, naba ejer batetan, etzanik arroka baten gañen.

Egun orano, bort'ondoko herri zoumbaitetan, haourak haren lotsa tuzu. Mouthiko edo neskatchar batek ama eztinin sinhetsi nahi, hounek erraiten diozu: "Abisa hadi, deitzen diat (ou diñat) Tantugou eta mementin jinen duk (ou dun) oihanetik".

Touan Germain, né à Barcus le 27 avril 1853 Coll.: Etchebarne, Maihegu, Salaber Beaudeant 


\section{Barbazaneco ichtoria}

Bacen lehenago Pyreneetaco herri batetan guiçoun bat eta emaste bat hanitch cahar cienac. Etcien etche chipi bat baratce bat eta behi bat baïcic. Bena, çoumbat nahi praube izanagatic, sokhorritcen çutien bethi hourac beno praube ago cienac. Egun batez, lurra elhurrez tapaturic cen, eta kharrountatcen ari cen harrien erdiratceco maneran. Herrico jentiac oro berotcen ari cien jatez eta edatez, eberax eta irous cien. Ulhuntcian bi bidajant hurruntic jiten cienac nahi izan ciren ukhuatu lekhu hartan, hotz eta gosse baitziren; jo cien bortha batetan, guero beste batetan eta beste elibatetan oraino: ihourc ere ez tcutien nahi ukhan sartcera ez eta her deus eman jatera. Orotaric casaturic, perseguituric hor gaitz elibatez, bi estranjerrec etçaquien nourat jouan, nouiz eta agitu beitcien herri basterrian, guizoun eta emazte praube cien hen etche aitzinian.

Entçuten çutialaric hen miseriac, senharra leihouan jarri cen hen deitceco, eta emaztia fite jouan cen bortharen çabaltcera. Galthatu gabe nour ciren, sar araci çutien eta jar eraci heen suthondouan; guero cerbitchatu ceyen ezne eta gaistagna çoumbait, cutienac oro. Bena ordian, goure Jauna -houra cen Jondane Phetiriequi,- jeiki cen, buru listreatcen cen batequi, eta erran ceren; "Ciec, praube cirie eta houn; cien aiçouac aberax dira eta gaichto. Jincouaren justicia eguin dadila!". Hain sarri, lurra ikharatu cen, Jesus eta Jondane Phetiri galdu cien eta bi çaharrac belharrico erori cien... biharamen goïcian, nouiz ere jouan nahi izan baitciren herrialat jakiteco cer igaran cen, etcien ikhousi etcheric batere: hen lekhian bacen lac bat, çouin edireiten beita oraino egun Barbazaneco eta Saint-Bertrand-de-Comminges hiriaren artian.

Behar dia laguntu beharrian direnac.

\section{Tantugouen ichtoria}

Tantugou çahar bat da çouin oyhanetan gordatcen beita. Beguiatcen tizu alhorrac eta sohouac, amoreacatic eta ez decen hetan deusere ebax. Nouiz ere ogiac picaturic beitira, eta belharra ebakiric, buruçaguiac oro bil artino, Tantugou ez ta batere lotcen; beillatcen da bethi. Haren ikhousteco loxac hurruntcen tu ouhouignac. Houn da, sinhex ezazu, bena salbaje da. Laborari gaiça hanitch caquian batec erraïten cian usu bere semer Tantugou cela segurki lurreco fruten beguiratcaleric hobena.

Uztailaco arrastiri batez, ardi tropa bat alha cen mendi batetan; artçaina lothu cen; iraçar ciarequi, apercebitu cian Tantugou lasterca jouaïten oyhanialat buruz. Eçaguturic içan cen ere artzagnez ibar ejer batetan, etçanic arroca batetan gagnen.

Egun oraïno, Pyrenéetaco herri çouimbaitetan, haurrac haren loxa dira. Nouiz ere muthico tchipi batec edo nescatchun tchipi batec ezpeitu nahi sinhexi bere ama, hunec erraïten deo: Gogua eman (si c'est une fille), Gogua emac (si c'est un garcon), deitzen diat (si c'est un garcon), deitcen dinat (si c'est une fille) Tantugou, eta behala jinen duc (à un garcon), jinen dun (à une fille) oyhanetic.

Jean Errécart, 26 ans, Irouléguy Coll.: Maître de Ayçaguer, ancien maire 
Bacen lehenago Pirene etaco herri batetan gizon bat eta emazte bat hanitch zaharrac. Etcien etche chipi bat, baratce bat eta behi bat baicik. Bena, zoumbat nahi praübe cien, sokhorritcen zutien berac beno praübigo cienac. Egun batez lurra elhurrez gordeik cen, eta kharrouk erdiatcen cin. Herrico jentik oro berotcen ari ciren, jaten eta edaten cielaic; aberatx eta irous ciren. Ulhuntcin, hurrunti jiten cien bi bidejant nahi zan cien baatu lekhu hartan, ceren hotz eta gosé beitcien; jo cien bortha bat guero besteik orano: ihourc ere etzutin nahi uken utci sartcera, ez deus eman jatera. Bazter orotaric akasaturik, zakhur gaitcez jaraikirik, bi estranjeek etzakien nouat joun, nouiz édién beitcién herriaen burin, praübe cien gizon eta emazte hayen etchiaen aitcinin. Hayen plenten entzutiarekin, senharra jarri cen leyhoun, hen deitceco, eta haren emastia fite jun cen borthaen zabaltcea. Galthatu gabe nour cien, sar-eaci zutien eta jar'aci beren suthondoun; gero zerbutchatu ezne eta gaztaña, hek ciena oro. Bena ordin, Goure-Jaüna - houra cen joundane Phetiri eki - jeiki cen, buria argi argia eta erran ceren, "Ciek, praübe eta houn cidie, cien aïzouk aberatx eta gaïchto dira. Jincouaren justicia egin dadila!". Hain tsari lurra ikharatu cen, Jesus eta joundane Phetiri galdu ciren, eta bi zaharrak erori ciren belhaïko....

Bihamen goïzan, herrilat joun nahi 'zan cienin, cer igaran cen yakiteco, etcien ikhousi etcherik batere, hayen lekhin, bacen losko handi bat, egun eano edieiten dena Barbazaneco herriaen eta Saint-Bertrand-de-Comminges-eko hiriaren artin. Behar dia laguntu malerousak.

$$
\text { oOo }
$$

Tantugou da gizon zahar bat zoiñ gordatzen beïta oïhanetan. Begiatcen tu alhorrak eta algatzik deus ere estecen ebatx. Ogic mouzturic eta belharrak ebakirik dienin, naüsik oro bil artino Tangugou ezta batere lotzen: bethi iratzariik dago. Haren aguertzen ikhousteko loxak etchekitzen tu ouhouiñak. Houn da, sinhetx ezazie, bena salbaje da. Hanitchko gaïza zakin laborari batek erraiten zin usu bere semer Tantugou zela segurki lurreko fruten begirariik hobena.

Arestiri batez, ustarilaco hilabetin, ardi saldo bat alhan ari cen bortin; artzaiña lothu cen: iratzartcin apercebitu cin Tantugou ezcapatcen oïhanilat burus. Bardin ezaguturik izan cen abere begirazale batzuez, naba ejer batetan, arroka batetan etzanik.

Egun oano, Pireneetaco herri zoumbaïtetan, haürrak loxa dira harentaco. Nouiz ere mouthiko edo neskatchouna chipi batek ezpeitu nahi bere amari obeditu, erraiten deo: Abisa hadi hiri! Tantugou deitzen diat (masculin) diant (féminin) eta istantin jinen duk (masculin), dun (féminin) oïhanetik!

B. Malet; 44ans, né à Garindein Coll.: Etchecopar, Lapeyre, 


\section{Barbazaneco ichtoria}

Bacen lehenago Pyreneetaco herri batetan guiçoun bat eta emazte bat hanitz cahar cienac. Etcien etche chipi bat, baratce bat eta behi bat baicic. Bena, çoumbat nahi praube içanagatic, sokhorritcen çutien bethi hourac beno praube ago cienac. Egun batez, lurra elhurrez thapaturic cen eta kharrountatcen ari cen harrien erdierazteko maneran. Herrico jentiac oro berotcen ari cien, jatez eta edatez; aberax eta irous cien. Ulhuntzian, bi bidayant hurruntic jiten cienac nahi içan ciren ukhuratu lekhu hartan, hots eta gosse baitciren; jo cien bortha batetan, guero beste batetan eta beste elibatetan oraino; ihourc ere etçutien nahi ukhen utci sartcera, ez eta her deus eman jatera. Orotaric cassaturic, perseguituric hor gaitz elibatez, bi estrangerec etçakien norat jouan, nouiz eta aguitu beïtcien herri bazterrian, guiçoun eta emazte praube cien hen etche aitcinian. Entçuten çutialaric hen miseriac, senharra leyhouan jarri cen hen deitceco, eta emaztia fite jouan cen bortharen çabaltcera. Galthatu gabe nour ciren, sar araci çutien eta jar araci hen suthondouan; guero cerbitchatu ceyen ezne eta gaistagna çoumbait, çutienac oro. Bena ordian, Gure Jauna -houra cen Joundane Phetiriequi- jeiki cen, buru listreatcen cen batequi, eta erran ceren: "Ciec, praube cirie eta houn; cien aïçouac aberax dira eta gaîsto. Jincuaren justicia eguin dadila!"

Hain sarri, lurra ikharatu cen, Jesus etea Joundane Phétiri galdu cien eta bi çaharrac belharrico jarri cien.

Biharamenian noïz eta ere jouan nahi içan nahi beitciren herrialat, jaquiteco cer igaran cen etciren ikhousi etcheric batere; hen lekhian bacen lac bat, cein edireiten beita oraino egun Barbazaneco herriaren eta Saint-Bertrand-de-Comminges hiriaren artian.

Behar dia laguntu maleroussac.

\section{Tantugouen ichtoria}

Tantugou çahar bat çouin oyhanetan gordetcen beita. Beguiatcen tu alhorrac eta sohouc, amoreacatic eta eztezen hetan deusere ebatx. Nuiz ere oguiac picaturic beitira eta belharra ebaquiric, buruçaguiac oro bil artino, Tantugou ez ta batere lotcen; beillatcen da bethi. Haren ikhousteco loxac hurruntcen tu ouhouignac. Houn da, sinhex ezazu, bena salbaje da. Laborari gaiça hanitch batec çaquian erraiten cian usu bere semer Tantugou cela segurki lurreco fruten beguiraçaleric hobena.

Uztailaco arrastiri batez, ardi tropa bat alha cen mendi batetan; arçaigna lothu cen: iraçar ciarequi, apercebitu cian Tantugou lasterca jouaiten oyhanialat buruz. Eçaguturic içan cen ere artçagnez ibar eiger batetan, etçanic arroca batetan gaignen.

Egun orano, Pyreneetaco herri çoumbaitetan, haurrac haren loxa dira. Nouiz ere mothico ttipi batec edo nescatila tchipi batec espeitu nahi sinhexi bere ama, hounec erraïten deo: Gogua emac (si c'est un garçon), Gogua eman (si c'est une fille), deitcen diat Tantugou, eta behala jinen duc oyhanetic.

J. Errécart, 26 ans né à Irouléguy Coll.: Edole maire et Goyheneix 
Bacen lehenago Piréné étaco herri batetan gizon bat éta emazte bat hanitch zaharrac. Ez cien etche chipi bat, baratce bat eta behi bat baïcik. Bena zoumbat nahi praübe cien, sokhorritcen zutien beran beno praübigo cienac. Egun batez lurra elhurez gordeic zen, eta kharrouk erdiatcen cin. Herrico jentic oro berotcen ari ciren, jaten eta edaten cielaric, aberatx eta irous ciren. Ulhun -tcin hurruntic jintencien bi bidejant nahi zan ciren baratu lekhu hartan, ceren hotz eta gose beitciren; jo cien bortha bat, guero beste bat, guero besteic eano; ihourkere etzutin nahukhen utci sartcera, ez deüs eman jatera. Baster orotaric kasaturik, zakhur gaïtcez jaraïkirik, bi estranjeek etz zakien nouat joun nouïz ere edien beitcien herriaren burin praübe cien gizon eta emaste hayen etchiaen aïtcinin. Hayen plinten entzutiarekin senharra jarricen leyhoun hen deitceco, eta haren emaztia fite joun cen borthaen zabalcea. Galthatu gabe nour cien sar-eaci zutien eta jar aci beren suthondoun; gero zerbutchatu ezne eta gastaña, hek ciena oro. Bena ordin, Goure Yaüna, (houra cen Joundane Phetiri ekin) jeiki cen buria argi argia eta erran ceren: "Ciek praübe eta houn cidie; cien aïsouc aberatx eta gaichto dira. Jincouaren justicia egin dadila.". Hain tsari lurra ikharatu cen, Yesus eta Youndane Phetiri galdu ciren, eta bi zaharrac erori ciren belhaïco. Bihamen goïzan, herrilat joun nahi san cienin cer igaran cen jakitera; et cien ikousi etcherrik batere: hayen lekin bacen losko handi bat égun éano edieiten dena, Barbazaneko herriaren eta Saint-Bertrand-de-Comminges-eko hiriaren artin. Behar dia laguntu malerousak"

\section{oOo}

Tantugou da gizon zaharbat zoïn gordatzen beita oïhanetan. Begiatcen tu alhorrak eta algatzik, deus ere estecen ebatx. Ogik mousturic eta belharrac ébakirik dienin, nausik oro bil artino, Tantugou esta batere lotzen, bethi iatzariik dago. Haren agertzen ikhousteko loxak etchekitzen tu ouhouiñak. Hound a, sinhetxezazie bena salbaje da. Hanitchko gaïza zakin laboraribatek eraïten zin usu bere semer: Tantugou zela zegurki lurreco frutin bégirariik hobena. Arestiri batez uztarilaco hilabetin, ardi saldo bat alhan ari cen bortin; artzaña lothu cen. Iatzartzin apercebitu zin Tantugou ezcapatcen oïhanilat burus. Bardin ezaguturic izan cen abere begirazale batzuez naba eïher batetan arroka baten gagnen etzanik. Egun éano, Piréné etaco herri zoumbaïtetan haürrak loxa dira harentaco. Nouïz ere mouthiko edo neskat-chouna chipi batek ezpeitu nahi bere ama obeditu, erraiten deo: Abisa hadi hiri! Tantugou deitzen diat (masculin), diat (féminin) eta istantin jinen duk (masculin), dun (féminin) oïhanetik

Lavie; âgé de32 ans né à Burgaronne 
Bazen lehenago Pyrenetaco heribatetan guizoun bat eta emazte bat hanix zahar zienak. Etzien etche tchipi bat, baratze bat eta behi bat baïzik. Bena zounbat nahi eskerniouan zien urgaïzten zutien bethi berak beno eskerniouago zirenak. Egun batez lura thapaturik zen elhurrez eta kharrounte zen harrien erdiazteko guizan. Heriko jentiak oro berotzen zien jatiareki eta edatiareki; aberax eta dohaxu ziren. Ulhüntzian bi bidajant hurruntik jiten zirenak baratu izan ziren lekhu hartan, zeren hotz eta gose beitzien; jo ziren borthabati, guero bestebati eta guero bestelibati orano: ihourk ere etzutien nahi ukhen utzi sartzera, ez eta ere deuserre her eman jatera. Ohilturik orotaric, jarraïkirik tchakur gaïtzez, bi arrotzek etzakien nouat jouan, nouiz ere khausitu beitzien heriaren bazterian, guizoun eta emazte eskerniouan zienen etche aïtzinian. Entzutiarekila haïen deïthuak, senharra jarri zen leïhouan haien deïtzeko eta harren emaztia jouan zen laster bortharen zabaltzera. Her galtheguin gabe nour ziren, sar erazi zutien eta jar erazi beren sutondouan; guero eman zeiïen ezne eta gaztagna zounbaït, zutienak oro. Bena ordian, Goure-Jaouna, - houra zen, Jondane Pethirieki, - jaïki zen buria osoki argui eta erraïten deie: "Ziek, eskerniouan zide eta houn, zien aïzouak aberax eta gachto dira. Jinkouaren justizia eguinik izan dadila!” Haïn sarri, lura ikharratu zen, Jesus eta Jondane Phetiri galdu ziren eta bi zaharrak erori belhariko. Biharamen goïzan, nouiz ere nahi izan beïtziren jouan herialat, jakiteko zer igaran zen, etzien ikhousi etcheik batere: hen lekhian bazen losko bat, egün orano edireiten dena Barbazaneko heriaren eta Saint-Bertrand-deCominjeko hiriaren artian. Behar die sokhoritu dohakabek.

oOo

Tantugou da zahar bat gordatzen dena oïhanetan. Beguiatzen tu alhorak eta sorhouak han deus ebax eztezen. Nouïz ere ogiak edo belharra ebakirik beïtira, oro thira artino buruzagiak Tantugou ez da lotzen batere; iratzaririk da bethi. Harren agertzen ikhusteko loxak tu etchekitzen ouhougnak. Houn da, sinhex ezazie, bena basa da. Laborari batek sougnek beitzakin hanix gaïza erraiten zian usu bere semer Tantugou zela segurki lureko fruten begirazalerik hobena. Uztailako arristiri batez ardi saldobat alha zen bortubatetan gagnen: artzagna lothu zen; iratzartziarekilan ikhousi zin Tantugou ezcapatzen oïhanilako gaïntilat. Izan tzen bardin ezaguturik artzagnetzaz naba eijer batetan etzanik botchu batetan gagnen.

Oano egün Pyrenetako heri zoumaïtetan haürak loxa dia harrentako. Nouïz ere muthiko tchipi batek edo nescatchouna batez ezpeïtu nahi sinhexi bere ama erraïten deïo: abisaïn hiri (pour une petite fille) abisaïk hiri (pour un petit garçon) deïtzen diant (pour une petite fille), deïtzen diat (pour un petit garçon) Tantugou eta, berhala, jinen duk oïhanetik.

Jean Arhanegoïty, né le 26 août 1868 á Lichans. Coll.: Pierre Etchecopar 


\section{Barbazaneco arraproua}

Bacen lehenago bortuco herri batetan guiçon bat eta emazte bat hanitz çahar cienac. Etcien etche tchipi bat, baratce bat eta behi bat baicic. Bena, çoumbat nahi praue cien, sokhorritcen çutien bethi beac beno beharrago cienac. Egun batez, lurra elhurrez gorderic cen eta harrien erdiatceco kharroua eguiten cian. Herrico jentiac oro beotcen cien, jaten eta edaten cielaric; abetx eta irous cien. Ulhuntcian, bi bidejant hurruntic jiten cienac ekhuatu nahi içan cien lekhu hartan, ceren hotz eta gosse beitcien; jo cien bortha bat, guero bestebat, eta beste elibat orano: ihourc ere etçutian sartcera nahi ukhen utci, ez eta etceren deusere jatera eman nahi ukhen. Hurrun igorriric orotaric, lastercaturic hor gaitz elibatez, bi arrotcec etçakien nouat jouan, nouiz ere aguitu beitciren herriaren bazterrian, guiçon eta emazte praue cien hen etchiaren aitcinian. Hen nahi gaben entçutiareki, senharra jouan cen leihouala hen deitceco, eta haren emaztia jouan cen bertan bortharen çabalcera. Nour cien galthatu gabe sar eraci çutien eta jar eraci ber suthondouan; guero eman ceren ezne eta gaztagna çoumbait, ciena oro. Bena ordian Jesukrist goure Jauna, -houra cen Joundan Phetirieki- jeiki cen, buria oro argui batez unguraturic, eta erran ceren: "Ciec praue eta houn ciradie; cien aïcouac abetx eta gaisto dirade. Jincouaren justicia eguin dadila!" Hain sarri lurra ikharatu cen, Jesus eta Joundane Phetiri galdu cien eta bi çaharrac jarri cien belharico.

Biharamen goician, herriala jouan nahi içan cienian, jakiteco cer iragan cen, etcien ikhoussi etcheric batere: hen lekhian bacen hour ekhuratubat, Sen-Bertran-Commingeco hiriaren eta Barbazaneco herriaren artian egun orano edireiten dena.

Beharretan dienec sokhorritu behar die.

\section{Tantugouen arraproua}

Tantugou oihanetan gordatcen den guiçon çahar bat da. Beguiatcen tu alhorrac eta sohouac deusere han ebax eztecen. Nouiz ere oguiac ebakiric beitira edo belharra daillaturic, buruçaguiaz oro sarthuric içan artino, Tantugou ezta batere lotcen; iratçarriric da bethi. Haren aguertcen ikhoustiaren loxac beguiratcen tu ouhougnac. Houn da, sinhex eçacie, bena salbaje da. Hanitz gaiça çakian laborari batec erraiten cian ussu bere semer Tantugou cela segurtameneki lurreco frutien beguiraçaleric hobena.

Uztarilaren hilabeteco arristiri batez, ardi saldo bat ari alhacen bortu baten gagnen; artçagna lothu cen; iratçartcian, ikhoussi cian Tantugou oihanialat ezcapatcen. Ber guisan içan cen artçagnez eçaguturic ibar ejer batetan, etçanic botchu batetan gagnen.

Egun orano, bortuco herri çoumbaitetan, haurrac haren loxa dira. Nouiz ere mothico tchipi batec edo urruchouna tchipi batec ezpeitu nahi sinhexi bere ama, erraiten dero; "Abisadi hiri! Deitcen diat (si c'est à un petit garçon qu'elle s'adresse), dignat (si c'est à une petite fille) Tantugou eta, behala jinen duc (à un garçon), dun (à une fille) oihanetic!"

Garat Arnaud, né à Abense de Haut, le 6 mars 1833 Coll: Salharanc Armand, Puchulu Jean, Uhart Jean et Etchecopar Pierre, expert 
Baçuçun lehenago Pyreneetaco heri batetan guiçon bat eta emazte bat hanich çaharturic cienac. Etcicien etche tchipi bat baatcé bat eta behi bat baïcic, bena çoumbat nhai praoubé ciren sokhorritcen cititcien berac beno praubiago cienac oro. Egun batez lura oro elhurez thapaturic çuçun, eta herien erdiratceco kharou bat baçuçun, herico gentiac oro beotcen çututçun jaten eta edaten cielaic, abeatx çututçun eta irous. Gaiaren hullantziareki, bi bidjant houna beitcien huruntic nahi içan çututçun ukhuatu guren hartan, ceentaco hostuic eta gosse beitcien; joiten dicie bortha batetan, guero bestebatetan eta beste çoumbaitetan ihourc ere etcititçun nahi ukhen utci sartcera, ez eta ere deusere eman jatera. Orotaric caçaturic, tchakhur gaistoues jerraïkiric bi estranjerec etçakicien nourat jouan, nouis eta ere ediren beitciren heriaren basterian, guiçon eta emazte praoube cien etchiaren aitcinian. Entçutiareki haïen plagniac, senhara jari çuçun leihouan haren deitceco, eta bere emaztia jouan çuçun berhala bortharen çabaltcera. Batere galthatu gabe nour ciren sar eraci citicien eta jar eraci suthondouan; guero cerbutchatu cieçun ezne eta gaztagna inkarubat besteric gabez. Bena ordian Goure- Jauna, houra beitcen Joundane Phetirireki, - jeiki çuçun buriac arguitcen cielaric eta erraïten dieçu: ciec praoube eta houn ciradie; cien aïcouac aberats eta gaïsto dira. Jincouaren justicia eguin bedi. Haïn sari lura ikharatu çuçun, Jesus eta Joundane Phetiri galdu çutuçun eta bi çaharac erori belharico. Biharamen goïçan, nahi içan cirenian jouan herialat, jakiteco cer igaran cen, etcicien ikhoussi etcheric batere: haïen lekhian itchasso tchipibat çuçun egun den beçala Barbazan heriaren eta Joundaen-Bertrand-Commigaco heriaren artian. Behar dicie laguntu malerousec.

(heldu den testua ez da esku berak egina)

$$
\text { oOo }
$$

Tantugou duçu guiçon çahar bat gordatcen dena oïhanetan. Beguiatcen tiçu alhorac eta sorhouac deuseren eez galtciagatic. Nouiz eta ere oguiac eta belharac ebakiric beitira, agniceta oro sarthuric içan artino Tantugou eztuçu batere lotcen, bethi beillan duçu. Haren ikhusten loxac etchekiten tiçu ouhougnac. Hun duçu sinhets eçaçu, bena salbaje duçu. Laborari hanits ikhassi cien batec eraiten ciçun bere semer Tantugou segurki lurreco frutien beguiraric hobena dela.

Uztaïlaco arastiribatez, ardi saldo bat alhan ciagotçun mountagnabateen alde gagnian; artçagna lothu çuçun; iatçarciareki ikhousten diçu Tantugou ezcapatcen oihanari buruz. Içan çuçun orano eçaguturic artçaïn elibatez ibar ejer batetan etçanic aroca baten gagnen.

Orano egun ere Pyreneetaco heri çoumbaitetan haurac haren lotsa tuçu. Nouiz eta ere mouchico tchipi bat edo uruchouna tchipi batec ezpadu nahi obeditu bere amari eraïten dioçu: Cassu eguic! deitcen diat Tantugou, eta berhala jinen duc oyhanetic. 


\section{Barbazanen ichtoria çaharra}

Bacien, beste orduz, Pyreneetaco herri batetan, guiçoun bat eta emazte bat hainitch çaharrac. Ez cien etcholat bat, baratce bat eta behi bat baicic. Bena, çoumbat nahi praue içanic, bethi laguntcen çutien berac beno praue agouac. Egun batez, lurra elhurrez gorderic cen eta harrien erdiratceco kharroua. Herrico jentiac oro berotcen ari cien eta ber demboran, jaten eta edaten; aberatx eta irous cien. Ulhuntciari, hurruntic, houna cien bi bidejant, nahi içan cien lehku hartan ukhuatu, ceren eta hotç eta gosse beitcien; jo cien bortha batetan, guero beste batetan eta orano beste elibatetan: ihourc ere ezçutian sartcera eitci nahi ukhen, ez eman mouchiic jatea. Orotaric igorriric, tchakur gaichtouac aztaletan, bi estranjerec ez çakien nouat jouan, nouiz eta ere heldu beitcien herriaen bazteriala, praue cien guiçoun eta emazt hen etcholaen aitciniala? Hen plaiñietara, senharra aguertu cen leihouala hen deitcea, eta emaztia laster borthaen çabaltcea. Galthatu gabe nour cien, sar eraci çutien eta suthondouan jar eaci; guero berhala eman ceren ezne eta çoumbait gaztaiña, çutienac, oro. Bena ordian, Jesu-Christ Gure jauna (ceren houa cen Jondane Phetiri eki), jeiki cen beguithartia argui arguia eta erran ceren: "Ciec, praue cirie eta jente hounac, cien aiçouac aldiz abeatx eta gaichto. Jincouac erakatx deçala bere justicia!" Ber mementouan, lurra ikhaatu cen, Jesus eta Jondane Phetiri galdu cien eta bi çaharrac belhaico erori... Bihamen goiçan, nahi içan cirienian jouan herriala, jakitea cer igaran cen, ez cicien etcheic haboro batere ikoussi; hen lekian, laco bat cen, orano egunco egunian agueri dena Barbazaneco herriaen eta San Bertran Commingaco hiriaen artian. Beharda heltu beharunian diener.

\section{Tantugouen Ichtoria çaharra}

Tantugou da guiçoun çahar oihanetan gordatcen den bat. Beguiatcen gu alhorrac eta sorhouac ezdadin deus ebatxic içan. Oguiac phicaturic dienian edo belharra ebakiic, naussiac oro bil artio Tantugou ezda lotcen; bethi iratçarriric dago. Haren aguertcen ikhoustiaen lotxac etchekitcen dutu ohoïnac. Guiçoun houna da, segur içaten ahal cirie, bena bassa. Laborari gaïça hanitch çakian batec ussu erraiten cian bere semer Tantugou cela dudaic batere gabe lureco uzten beguiaiic hobeena.

Uztarilaco arristiri batez, arres saldo bat alha zen mendi batetan; louac hartu cian artçaiña: iratçartciaeki, ikhoussi cian Tantugou lasterca oihanialat buruz. Halahala, eçaguturic içan cen beste artçaiñ elibatez, ordoki ejer batetan botche baten gaiñen etçanic.

Orano egunco egunian, Pyreneetaco herri çoumbaitetan, haurrec haren lotxa badie. Ala mothico, ala nescatila, beren ama sinhexi nahi ez dienian, aski dere erraitia: Abisa hadi! Deithcen diat Tantugou eta lasterca jinen duc oihanetic!

Duleau, 26 ans, né à Etchebar Coll.: Laurent Puyade 
Bacen lehen Pyrénéetaco heri batetan guiçon bat eta emaste bat, çouiñ beitciren hanitch çahar. Etcicien etche tchipi bat, baratce bat eta behi bat baicic. Bena, çoumbat nahi praübe ciren, socoritcen citicien bethi houac beno praübeago cienak. Egun batez, lurra elhurez gorderic çuçun, eta kharou eguiten ciçun harrien erdiatceco guisan. Heriko jentiac oro berotcen çutuçun, jaten eta edaten ari cielaic. Aberac çutuçun eta irous. Ulhun çartcin, bi bidejant çouiñ houna beitciren huruntic, nahi içan çutuçun ukhuatu lekhu hartan, ceren gosse eta egari beitciren. Bortha bat jo cicien, guero beste bat eta besteric orano. Nihourc etcitiçun nahi utci sartcera, es deus eman jatera.

Acassaturic orotaric, jeraikiric tchachur gaïtz eli bates, bi arrotcek etçaquicien nouat jouan, Nouis eta heldu beitciren heri basterila, guicoun eta emaste praübe cienen etché aitcinila. Hayen plagniac entçunic, senhara jari çuçun lehouan hayen deitceco, eta haren emastia fité jouan çuçun bortharen çabalcera. Galthatu gabe nour cien, sar eraci citicien eta jar eraci suthondouan: guero cerbutchatu cieçun esné eta gastagna çoumbait, çutienac oro. Bena ordian, Gouré Jaunan -houra çuçun- Joundané Phetirequi,- chutitu çuçun, buria oro arguituric eta erran cieçun: "Ciec, praübe cide eta houn; cien aisouac aberax tuçu eta gaisto. Jincouaren justicia eguin dadila!" Haiñ tsari, lura ikharatu çuçun, Jésus eta Joundané Pheti galdu çutuçun eta bi çaharrac erori belharico.

Biharamen goician, nouis éré nahi içan beitciren jouan herila jaquiteco cer igaran cen, etcicien ikhoussi etcheric batére. Hayen plaçan, baçuçun losko bat, egun orano edireiten dena Barbazaneco heriaren eta St Bertrand de Comminges hiriaren artian.

Behar dicie laguntu malerousec.

oOo

Tantugou duçu guiçun sahar bat çouin gordatcen beita oihanetan. Nouiz éré oguiac phicaturic beitira edo belharac ebaquiric, oro naüssiac sar artino, Tantugou estuçu lotcen batere: beillatcen duçu bethi. Haren ikhousteco loxac beguiratcen tiçu ouhougnac. Houn duçu, sinhets eçacu, bena salbage duçu. Laborari, gaiça hanitch çakin batec, eraiten cieçun ussu béré semer Tantugou cela segur lureco frutien beguiraçaleic hobena.

Ustarila hilabeteco aristi batez, ardi tropa bat alha çuçun mendi batetan. Arçaigna louac hartu ciçun; iratçartsiarequi ikhoussi ciçun Tantugou çougnac ihessi eguiten beitcian oihanialat. Içan çuçun bardin eçaguturic ulhagnetçaz naba ejer batetan, etçanic arroca baten gagnen.

Orano egun, Pyrénéetaco heri çoumbaitetan, haürrac haren beldur tuçu. Nouis ére mothico tchipi batec edo neskatila tchipi batec espeitu nahi obeditu bere amari, hounec eraiten dioçu: Abisa hadi, hi! Deitcen diat Tantugou, eta berhala ginen duk oihanetic. 


\section{Barbazaneko iracourgaria}

Baçuçun lehenago Pyrenetaco herri batetan giçon bat eta emazte bat couin hanitch çahar beitcien. Etcicien etche chippi bat, baratce bat eta behi bat baicik. Bena çoumbat nahi praoube içana gatik, bethi sokoritcen citicien berak beno praoubigo cirenak. Egun bathez lurra elhurrez gordeik çuçun eta karrou eguiten ciçen harrien erdiatceko puntin. Herriko jentik oro berotcen çututçun - jatieaeki eta edatiaeki; abeats çututçun eta irous. Iluntciai, bi bidajant çougnak hurruntik horra beitcien nahi içan çututçun baratu leku hertan, ceren eta hotz beitcien eta gose; jo cicin borta batetan, gero beste batetan eta beste hanitchetan oano: nihourkec etciticien sarcea utci nahi ukhen, ez etae jatea eman nahi ukhen. Baster orotaik kasatuik, persegituik chacur gaitzez, bi estranjeek etçakicien nouat joun, nuizee herriko beste buila heldu beitcien, praoube cielako giçon eta emazte heen etchiaen aincinila. Heen plagnien ençutiai, senharra leihoun jari çuçun heen deitceko, eta haren emaztia joun çuçun fite bortaren çabalcea. Galdegin gabe nour cien, sar aci cititcien eta sutondoun jar aci; gero cherbitchatu cieçun ezne eta çoumbaït gaztegna, heek ciena oro. Bena ordin, Goure Salbaçalia -houra çuçu, jondane Petiriekin - jeiki çuçun buria argi argia eta erran cieçun "Siek praoube eta houn cide; cien aiçouk aberats eta gachto. Jinko hounaen juzticia egin dadila!" Hain sari lurra ikharatu çuçun, Jésus eta Jondane Petiri galdu çututçun eta bi çaharrac belhaiko erori çututçun. Bihamun goiçan, nouizee joun nahi içan beitcien herilat, jakiteko cer igan cen, etcicien ikousi etcheik bate; heen lekin, baçuçun losko handi bat, Barbazaneko heriaen eta St Bertrand-de Comminjeco hiiaren artian egun oano edieiten den houa. Laguntu behar dicie malerousek.

\section{Tartaouaen iracourgaria}

Tartaroua duçu giçon çahar bat couin oihanetan gordatcen dena. Begiatçen titçu alhorrak eta sohouk deusee hen ebats eztecen. Nouize, ogik mouzturik beitia edo belharrac ebakhik, oro buraçagik bilduik içan artino, Tartaouk eztiçu bate loik egiten; beleatz dagoçu bethi. Haen agercen ikousteko lotsak begiatcen titçu ouhougnak Houn duçu, sinhets eçaçu, bena Salbaje duçu. Laborari batek çougnek beitçakin gaiça hanitch usu erraiten ciçun Tartaoua cela segurki lurreko frutien begiraçaleik hobena.

Uztailaren ilabeteco arrasti bates, ardi saldo bat alhatcen ai çuçun mendi baten gagnen; arçagna lothu çuçun: iatçarciai aperbecitu ziçun Tartaroua ihez jouiten beitcen oihanilat buus. Eçagutuik içan çuçun bergijan arçagnez arbailla ejer batetan, etçanik aroka baten gagnen.

Oano egun Pyrenetako herri çoumbaitetan haourak haren lotsa tutçu. Nouize moutiko chippi batek edo neskatila chippi batek ez beitu sinhetsi nahi bere ama, erraiten dioçu: Casu egineçak hiri! Deitcen diat Tartaroua eta behala jinen duk oihanetik. 


\section{Barbazaneco khountu saharra}

Bazen lehenago Pyrénéetaco herri batetan gizon bat eta emazte bat hanitz sahar zienak. Etzien etché chipibat, baratze bat, eta behi bat baisik. Bena zoumbat nahi praübe isanic ere sokhoritzen sutien bethi berac beno praübigouak. Egun batez, lurra gorderic sen elhurrez eta kharrouak erdiatzen zin. Herrico jentic oro berotzen ari siren jatiarekin eta edatiarekin: aberax sututsun eta irous. Ulhuntzin hurruntic jitenciren bi bidejan nahisan zien arrestatu lekhu hartan seren gosse eta égarri beitziren; jo cien borta batetan, gero beste batetan eta orano beste sumbatetan, ihourk ere etzutien nahi ukhen utzi sartzera es eta ere deuse eman jatera. Ohilturik bazter orotaric, jarraikirik hor malezian sien elibatez, bi arotzek etzakien nourat jouan nouiz eta heltu beitziren herriaren bazterrin, gizon eta emazte praübe harien etchiaren aitzinin. Hayen plagnien entzutiari senharra jarri sen leihoun hen deitzeko eta emaztia jouantzen bertan bortharen zabaltzera. Her galthatu gabetarikk nour siren sar eaci zutien eta jar erassi bere suthondouan gero serbutchatu seyen ezne eta gastegna soumbait sutinac oro. Bena ordin Goure-Jaüna -houra cen Jundane Phetirieki- Jeiki sen buria argi argia eta erran ceyen; "Ziec praübe eta houn zidie, zien aizouk aberatx eta gaisto dira. Jincouaren justicia egin dadila!" Haïn sarri lurra ikharatu sen, Jesus eta Joundane Petiri galdu siren eta bi saharrak erori siren belhaïko... Biharamun goizan joun nahi izan sirenian herrila jakiteco ser igaran cen etzien ikhoussi etcheric batere: haien lekhin basen losko handi bat sougna ere edireiten beyta orano egun Barbazaneco herriaren eta Saint-Bertrand-de Commingeco hiriaren artin, Behar dira laguntu malerouzak.

\section{Tartaüaren khountu saharra}

Tartaüa gison sahar bat da oïhanetan gordatzen dena. Bégiatzentu alhorrak eta sorhouak amourekati han deusere ebatx eztezen. Nouiz ere ogiak mouzturic beitira edo belharrak ebakiric, oro naussiz sarturik içanartio Tartaüa ezta batere lotzen; iratzarririk dago bethi. Haren ikhousteko lotxac erretenitzentu ouhougnak. Houn da, sinhex esasie, bena salbaje da. Gaiça hanitz sakin laboraribatec erraiten sin usu bére semer: Tartaüa sela segur lurreko frutien beguiraririk hobena. Uztailako arrestiri batez ardi saldobat alhan ari zen bortu baten gagnen; artazagna lothu zen, iratzarsiarekin ikhousi cin Tartaüa oihanilat eskapatzen. Essaguturik izantzen bergisan artzagnez arbailla ejer batetan, aroka baten gagnen etzanic.

Orano egun Pyrénéetako herri soumbaitetan haürrac haren lotsa dira. Nouiz ere mothico chipi batec, edo nescatila chipi batec ezpeitu nahi obeditu bere ama hounek erraiten deyo: Khassu egin eçak hiri! Deitcen dut Tartaüa, eta haïn sarri jiten da oïhanetik.

J. Barrenne; 28 ans, Montory Coll.: M. Laplume 


\section{Barbazanen iztoria}

Bacen lehenago Pyrenetaco heri batetan guiçonbat eta emazte bat çouin hanitz çahar beitcian. Etçuten etche tchipi bat, baratce tchipi bat eta behi bat baïcic. Bena çoumbat nahi praube cien, sokhoritcen çutien bethi berac beno praubiago cienac. Egun batez, lura thapaturic cen elhurez, eta kharou eguiten cian harien erdiazteko guisan. Herico gentiac oro berotcen ari cian, jaten eta edaten cielaic; aberax eta irous cien. Ulhuntcian, bi bidejant, çouin huruntic jiten beitciren, nahiçan cian arastatu lekhu hartan, ceren gosse eta hotz beician; joïten die bortha bat, guero beste bat eta beste elibat orano; ihourc ere etçutian utci nahi ukhen sartcera, ez eta deusere eman nahi ukhen jatera. Orotaric cassaturic, ho gaïtz eli batez perseguituric, bi estranjerec etçakiten nourat jouan, noiz eta edireiten beitira heriaen bazterian, guiçon eta emazte praube hen etche aïtcinian. Hen plagnien entçutiarequi, senhara jouaïtenda leihoula hen deitceco, eta emaztia jouan cen fite bortharen çabaltcera. Batere galtheguin gabe nour ciradien, sar eraci çutien eta jar eraci beren suthondouan, guero cerbutchatu ceren ezne eta gaztagna çoumbaï, hec çutena oro. Bena ordian Jinco hounak, - eci houra cen Jondane Phetirieki,- jeiki zen buria arguituic eta eran ceren: "Ciec praube eta houn cide; cien aïçouac aberax eta gaïzto dira. Jincouaren justicia eguin dadila". Berhala lura ikharatu cen, Jesus eta Jondane Phetiry bistatic galdu ciren; eta senhar emazte çaharac belharico erori cien. Bihamen goïcan, nahiçan cienian jouan herila jakiteco cer igan cen, etçuten ikhoussi etcheric batere: hen lekhian bacen ossin handibat, orano egun edireiten dena Barbazan eta Saint-Bertrand-deComminges deitcen dien bi herien artian. Behardia sokhoritu malerousac.

\section{Tantugouen iztoria}

Tantugou guiçon çahar bat da çouin gordatcen beita oïhanetan. Beguiratcentu alhorac eta sohouac, han ihourcere ebax ezteçan deusere. Nouiz eta oguiac mousturic beitira edo belhara ebakiric, oro bil artino buruçaguiac, Tantugou ezta batere lotcen; iratçariric dago bethi. Haren lotxac, beguiratcen tu ouhougnac. Houn da, sinhex eçacie, bena bassa da. Laborari batec, çougnec beitçakian gaïça hanitch, eraïten cian ussu bere semer, Tantugou cela segur lureco frutien beguirariric hobena.

Uztaillaco aristiri batez, ardi saldo bat alha cen bortu baten gagnen; artçagna lothu cen; iratcarciareki ikhoussi cian Tantugou escapatcen oïhanilat. Içan cen ber guisan eçaguturic artçagnez naba ejer batetan, etçanic haroka batetan gagnen.

Orano egun ere, Pyrenetaco heri coumbaitetan, haurac loxa dira harentaco. Nouiz eta mothico tchipi batec edo neskatila tchipi batec, ezpeitu nahi obeditu bere amari, amac eraiten do: Abisaic; deitcen diat Tantugou; eta berhala jinen duc oïhanetic.

B. Laxague, 49 ans, né à Ossas-Suhare

On n'a pas pu trouver un seul individu dans la commune en état de nous donner des renseignements de quelque importance 


\section{Barbazaneco kountu sahara / Barbazaneco istoria}

Bacien besteorduz Pyreneetaco herribatetan guiçon eta emaztebat hanitch chararac. Etsien etchetto bat, baatsia eta behitto bat baicic. Bena, nahi bada praube, berac beno beharagouer bethi heltura eguiten cien. Behin arren, lurra elhurrez oro gorderic sen, harriaren hausteco kharoua ari sen. Herrico gentiac oro suthondouan ciren jaten eta edaten; aberatx éta irous ciren. Ulhuntciari, hurrunti jiten cien bi bidajant, lekhu hartan nahi içan ciren ostatatu, cereneta hotz eta gosse beiciren; jo ciren aren bortha bat, guero beste bat eta beste asko: nihourcek etsein nahi ukhen çabaltu ez eta ere eman jatera deuzere. Orotaric ohiltuik, chakur gaïstouez perseguituric, bi arotzek etçakien nourat utçul, nouiz eta herriaren basterila heltu beitcien, guiçon eta emazte behar haien etche eretcila. Haien deithoamenac entçunic, senhara leihoula aguertu cen haien deitceco eta emaztia laster joun seien bortharen çabalcea. Galthatu gabe nour ciren, çareraci çutien eta jareraci çuçulian (suthondouan); guero, serbutchatu ceien ezne eta gaztagnac, çutien erekeitiac. Bena ordin, Salbaçaliac, - eci houra bera cen Joundane Phetirieki- chutitu cen, buria arguidua handi batez unguratuic, eta erran ceien: "Cirec praube eta houn cidie; ciren aiçouac aberax eta gaisto dira. Içan bedi Jincouaren justicia!”. Haen sari, lurra ikharatcen da, Jesus eta Joundane Phetiri galcen dira, eta bi chaharac belharicatzen dira.... Bihamen goiçan, joun nahi içan cirenin herrila, cer çabilan jakitera, etcien ikhousi etcheric batere; haien lekhian, bacen ordari losko çabalbat, egun orano edieiten dena Barbazaneco herriaren eta Saint-Bertran-Comminjeco hiriaren artin.

Horek erran nahi du beharer heltua behardela éguin.

(ou Beharda laguntu malerousac)

\section{Tantugouren istoria}

Tantugou da oïhanetan gordatcen den guiçon chaharbat. Alhorac eta sohouac beguiatcen dutu ebaxletaric. Oguiac eta belharrac ebakiric dienin, naussiac bilartio, Tantugou ezta lo; bethi iratçariric dago. Haren batceco beldurrac beguiatcen dutu ouhouñac. Guiçon houna da, bai segur, bena bassa da. Hanitch çakien laborari batec, sion ardura bere semer, Tantugou sela segur lurreco uzten beguirari hobena.

Ustailaco aristi batez, arez saldo bat alha sen mendin: artçaiña lothu sen: iratcarciaeki, Tantugou ikhoussi sin ihessi jouiten oïhanilat. Beste artçain elibatec ere eçagutu cien houra sela, aroka gaĩn batetan, etçanic, ibar ejerbatetan.

Egun orano, Pyreneetaco herritto elibatetan, haren beldura badie haurec. Muthico batek edo uruchouna batec sinhetsi nahi eztienïn bere ama, eraïten deo: "Abizaic! Deitcen diat Tantugou eta, berhala, jinen duc oïhanetic!

J. Bordenave, 27 ans, né à Baigts, Basses Pyrénées Coll.: Etchebarne, Althabe, Duhalt 
Baçuçun nouizpait goure Bortu ondo hayetaco herri batetan guiçoun bat eta emazte bat hanix çaharturic. Etcicien etche ttipibat, baratce eta behi bat baïcic. Bena, çoumbat nahi beharrac ciren; eguiten cicien bethi heltura bera beno beharretan ago cirener Egun batez, lurra elhurrez thapaturic çuçun, eta kharrountatcen ari çuçun harrien erdiratceco aski. Herrico gentic oro berotcen ari çutuçun, jatez eta edatez; aberax eta irous çutuçun. Ulhuntcin, bi bidageant couiñ hurruntic horra beitciren, nahi içan çutuçun gune hartan ukhuratu ceren eta egarri eta gosse beitciren; jo cicien bortha batetan, guero beste batetan, eta beste çoumbaitetan orano: ihourc ere etciticien nahi ukhen utci sartcera, ez deusere her eman jatera. Orotaric igorriric, hor ehelegatu elibat ondoun jarraikiric, bi arrotzec etçakicien nourat joun, nouiz eta ediren beitciren kharrica bazterrin, guiçoun eta emazte behar hen etche aitcinin. Hen plagnien entçutiareki, senharra leihoulatu çuçun her oyhu eguiteco, eta emaztia jouan çuçun bortharen çabaltcera. Galthatu gabe nour ciren, sar eraci citicien eta jar eraci su pphazterrin; guero eman ciecien ezne eta gaztagna, hec ciena oro. Bena ordin, Goure Jauna (Jesus-Crist), ecic houra çuçun, Joundane Phetirireki- chutitu çuçun, buria arguidura batec harturic, eta erran ciçun; "Ciec praube et houn ciradie; cien aiçouac aberax tuçu eta gaichto. Gincouaren justicia eguin bedi!" Haimbestereki lurra ikharatu çuçun, Jesus eta Joundane Phetiri galdu çutuçun eta bi guiçoun emazte çaharrac belharico erori çutuçun... Biharamen goiçan, nouiz eta nahi içan beitciren joun kharricala jakiteco cer igaran cen, etcicien ikoussi etcheric batere; hen lekhin baçuçun losco bat, egunco egunin orano edireiten dena, Barbazaneco herriaren eta Sen Bertrand Commingeaco hiriaren artin. Behar diçu heltura eguin malerouser.

\section{oOo}

Tantugou duçu guiçoun çahar bat couiñ gordatcen beita oyhanetan. Beguiratcen tiçu alhorrac eta sorhouc han deusere ihourc ebax eztezen. Nouiz eta oguic edo belharra ebakiric beitira, agniketa hourac oro buruçaguic barnerat bil artio, Tantugou eztuçu batere lotcen; beillatcen duçu bethi ou bien beilheratz egoiten duçu bethi. Haren aguertcen ikhousteco lotsac baratcen tiçu ouhougnac. Galant duçu, sinhexi behar ducie, bena bassa duçu. Laborari batec hanix gaiça çakinak erraiten cieçun ussu bere semer ecic Tantugou cela segur lurreco uzten beguirariic hobena.

Uztailako arristiri batez, ardi saldo bat alha ari çuçun mendi gaiñ batetan: artçagna louac hartu ciçun: iratçartciareki asmatu ciçun Tantugou ihessi jouiten oyhanilat buruz. Guisa berïn ecaguturic içan çuçun artçagnez arbailla ejer batetan, etçanic botchu batetan gagnen.

Orano egunco egunin, goure bortu ondouetaco herri coumbaitetan, haurrac harren lotsa tuçu. Nouiz eta mouthico edo nescatchou ttipi batec ezpeitu nahi obéditu bere amari, hounec erraiten diroçu: Abisa hadi! Tantugouri oyhu eguiten diouat ou bien diognat, eta, mementin, jinen duc ou dun oyhanetic 


\section{Barbazaneko khountu çaharra}

Baçuçun lehenago Pyreneetaco heribatetan guiçon bat eta emazte ba hanitch çahar beytciren. Etcicien etche ttipi bat, baratce bat eta behi baycic. Bena, çoumbat nahi praube içanagatic, sokhorritcen cititcien bethi berak beno praube ago cirenac. Egun batez, lura elhurez gorderic çuçun eta kharoua hanitch cinez ari çuçun. Heriko gentik oro berotcen çututçun gaten eta edaten ari cirelaik. Ulhun ceratcin, bi bidejant huruntic hora cienak, baratu nahi içan çututçun lekhu hartan, ceren eta gose eta hotz beytciren; jo cicien bortha batetan, guero bestebatetan eta beste elibatetan orano: ihourk erek etcititcien sartcera utci nahi uken ez eta ere eman deusere jatera. Bortchaz igoriric bazter orotaric, lasterkaturik chakhur gaïtz elibatez, bi estranjerek etçakicien nourat joun nouis eta heltu beytciren heri bazterila, guiçon eta emazte praube hayen etche aitcinila. Hayen plagnien entçutiarekin, senhara leyhoula aguertu çuçun haien deytceko eta emaztia fite joun çuçun bortharen çabaltcera. Batere hayer galthatu gabe nour ciren, sar eaci cititcien eta jar eaci suthondo chokhoun; guero cerbutchatu cieçun ezne eta gaztegna çoumbaï, hayek cirena oro. Bena ordin goure Ginco Jauna -houra çuçun Saint Pierra ekin- chutitu çuçun buria argui arguia eta eran cieçun: "Ciec praube eta houn cidie, ciren ayçouk aldiz aberatz eta gaychto. Gincouaren justicia eguin dadila” Hagn sari, lura ikharratu çuçun; Jesus eta Saint Pierra galdu çututçun eta bi jente çaharrak belhayko erori. Bihamen goyçan, herila joun nahi içan cienin, jakiteko cer igaran cen, etcicien ikhousi etcherik batere: hayen lekhin baçuçun losko edo hour handi bat, egun edireyten dena orano Barbazan eko heriaren eta Saint- Bernard-de-Cominges eko hiriaren artin. Behar dia laguntu malerusak.

\section{Tantugou en khountu çaharra}

Tantugou duçu guiçon çahar bat gordatcen dena oyhanetan. Beguiratcen titçu alhorac eta sohouc, amorekatik deuzere han ebatz estecen. Nouiz ere belhara edo oguik mouzturik beytira, Tantugou eztuçu batere lotcen; buruçaguik oro elkhartino; bethi iatçaririk duçu. Haren aguertcen ikhousteko lotsak ouhougnak baratcen titçu. Houn duçu, sinhesten ahal ducie, bena salbaje duçu. Laborari, gaiça hanitch çakin batek, erayten ciçun usu bere semer Tantugou cela segurki lureko fruitiren beguiraçalarik hoberena.

Uztaillako aresti batez, ardi saldo bat alhatcen ari çuçun bortu batetan; artçagna louac hartu ciçun; iratçartcin ikhousi ciçun Tantugou oyhenilat buruz ezkapatzen cela. Bergaïça eçaguturik içan çuçun beste kabale beguiraçale elibatez arbailla eyjer batetan, aroca batetan gagnen etçanik.

Pyreneetako herri çoumbaïtetan, egun orano, haurak haren tako lotsa tutçu. Nouiz ere mothiko edo neskatchouna ttipi batek espeytu nahi bere ama obeditu, erayten dioçu: "Khasu eguin eçak hiri (ou bien si l'on parle à la fille) khasu eguin eçan hiri! Deytcen diat (ou bien) deytcen dinat (selon que l'on parle à un petit garçon ou à une petite fille) Tantugou eta berhala ginen duk ou ginen dun oyhanetik 


\section{La légende Barbazan}

Bacien lehenago Pyrenetaco herri batian guiçon bat eta emazte bat araz çahartiac. Ez cuten etche ttipi bat, baratce bat eta behi bat baïcic. Baïnan probe içana gatic, bethi laguntcen ciuzten berac bano probiago cirenac. Egun batez, lura estalia cen elhurrez eta horma eguiten cien harrocac erdiatceco anocoan. Herrico yende guciac berotcen cien yatian eta edatian, aberax eta uroz cien. Ilhun nabarian, bi pidayant heldu cirenac urunetic leku hartara, nahi çuten guelditu leku hartan ceren eta gosse eta egarri beitciren, yo çuten borta batian, guero bertce batian eta bertce batcutan ere: nihorc ez ciuzten sartcera utci nahi içan ez eta deusse eman yatera. Cassatiac orotaric, seguitiac çakur garassia eguile batcuz, bi estranyerec ez çakiten norat joan, noiz eta ere caussitu beitçuten herriaren bazterian guiçon eta emazte probe cien hen etchiaren aintcinian. Hen pleïniac aytcearekin, senhara plantatu cen leyhoan hen galdeguiteco eta haren emaztea yoan cen ussu bortharen çabaltzera. Batre her galdeguin gabe nor ciren sar aci ciuzten eta yarraci ciuzten beren su pazterian; guero cerbitçatu ceeten esnia eta çombait gaztena, ciuzten guciac. Bainan Yesu Cristo gure Yauna - hura cen Yondoni Petrikin- alchatu buria dena distirant eta eran ceen: Ciec probiac ciezte eta hunac cien aucoac aberaxac eta gaïchtoac. Yincoaren yusticia eguin bedi!" Berehala lurra ikarratu cen. Yesus eta Yondoni Petri bichtatic yoan cien eta bi çaharrac erori cien belhaünico. Biharamun goician, noiz ere nahi içan baitcuten yoan herrirat yakiteko cer passatu cen, ez çuten ikussi etcheric batere: hen tokian (ou lekian), bacen hur gueldi handi bat, egun oraîno caussitcen dena Barbazeneco eta Saint Bertrand Cominyeco hiriaren artian. Behar dira lagundu malorossac.

\section{La légende de Tantugou}

Tantugu da çahar bat gordetcen dena oyhanetan. Çaintcen tu alhorrac eta peintciac ez deçaten deus ebax. Oguiac picatiac direnian edo belharrac ebakiac, denac kendiac ican artio naussiaz, Tantuguc ez du loïc batre eguiten, goardian dago bethi. Haren aguertcen icustiac atchikitcen ditu ohoïnac. Huna da, sinhex acie, bainan salbaya da. Laborari asco gaüca cakien batec eraiten cien ardura bere semer Tantugu cela segurki lurreco fruitien beguiraçaleïc hoberena.

Uztailaco axalde batez acinda tropa bat alhan ari cen mendibaten gainian, artçaïna lokartu cen, iatçartciarekin hauteman cien Tantugu ihes eguiten ciela oyhanari buruz. Içan cen igoal eçagutia artçainez celhaï pollitbatian etçana harroca baten gaïnian.

Oraïno egungo egunian, Pyrenetaco herri batçutan, haurec beldur dute harendaco Motico ttipi edo nechcato ttipi batec obeditu ez direnian nahi bere amari erraiten dee: Goardia emac (masc. à un petit) goardia eman (au féminin) hiri ekarazten dut Tantugu eta ichtantian yinen da oyhanetic!! 
Bazen lehenago Pyrenetaco herri batian guizon bat eta emazte bat biziki chaharrac zienac. Etzuten etche ttipi bat, baàtze bat eta behi bat baizik. Bainan hain beharrac izanic ere, sokhorritzen ziousten berac baino beharraouac. Egun batez lurra estaliazen elhurrez eta kharrogna arizien borthizki. Herrico yende guziac berotzen zauden, yaten eta edaten arizirelaik: aberats eta ouros ciren. Ilhuntzian, bi piayant heldu cienac urrunetik nahi ukhan zuten arrastatu lekhu hortan, ceren hotzac eta gosiak baitzien, yozuten bortha bati, guero beste bati eta oono beste batzuer: nehorc etziusten sartzerat utzi nahi izan, ez eta ere deus ere eman yaterat. Khasatiac orotaik, persegitiak chakhur gaichtobatzuz, bi estranyerrec ez zakiten norat yuan, guerthatzen dielaik herriain beste burian, guizon eta emazte behar ciren heèn etchiain aitzinian. Intzutiaikin heèn plegniac, senharra yartzenda leyhouan heén deitzeko, eta hain emaztia yuanzen fite borthain idekitzeat. Galdin gabe nor ciren, sarraazi ciusten eta yarraàzi beren subasterrian; guero cherbitzatu zuten esnia eta zombait gaztagna, ziuzten guziac. Bainan ordian, Gure Yauna - hurazen Yandone Petriikin - yeikitzen da, buria argui arguia, eta erten deè: "Ziek beharrak ziizte eta hunac; zien auzuak abeatsak eta gachtouak. Yainkouain justizia ina izandaila!" Ordian berian lurra ikharatzen da, Yesus eta Yandoné Petri ezestatzen dira, eta bi chahar hec erortzen dira belhauniko. Bihamoun goizian, nahi izan zutelaik yuan herrirat yakiteko zer phasatuzen, etzuten ikhusi etcheric batere: heèn lekhian bazen leize bat zoina kausitzen baita oono egun Barbazaneko herriain eta San-Bertrand- Commingeko hiriain artian.

Behardira sokhorritu malhurosac

\section{Tantugouin}

Tantugou da guizon chahar bat oyhanetan gordetzen dena. Zaintzentu alhorrac eta phenciac nehoc deuzein ez ébasteko. Oguiac eta belharrac pikhatiac diélaik, naoussiak erretiratu artio, Tantugou ez da lokhartzen, bethi iatzarik dago. Haïn aguertzeko beldourrac atchikitzentu ohoinac. Huna da, sinétsazié, baïnan bassa da. Laborari gauza anitz zakien batec ertenzien ardura bere semer Tantugou zela séourki lurreko fruitien beiratzaléik hobééna.

Uztailleko atsalde batez, ardi tropa bat alhatzen zen mendi batian, artzaïna lokhartuzen: iatzarziaikin, ikhusten du Tantugou ihesi zuala oyhaneko alderat. Igual ezaoutia izantzen behi zaïnez erreka pollit batian, etzana harri pegna baten gainian.

Ono egun, Pyrénétako herri zombaitetan, haurrec haren beldurra badute. Mutikho ttipibatec edo nechkato ttipibatec ez dutelaïk nahi beren ama obeditu, erraiten déè hunek: Gouardimac! Oihu iten diat Tantugouri, eta ichtantian, yinen duc oyhanetic!

Sallaberrry, 28 ans, né à Saint-Jean-Pied de Port. 
Bazi'en lehena'u Pyrenetaco herri batian guisom bat eta emaste bat arrajs saharrac. Etche ttipi bat, baratze bat eta behi bat besic ez t'zuten. Bainan sembat êê pobriac baitsien, berac baino aphalaguac laguntzen siusten. Egun bates lurra elhurres ejstalia sen, eta horma e'iten sien harriac arrailtseco pundian. Herrico jende gusiac jaten eta edaten ari si'elay'ic, berotsen siren, abaratxac eta urojsac siren. Ilhuntzian bi piajiante urrunic jiten si'enac, herri hartan nahi isan suten guelditu hotsac eta gojsiac baitsi'en. Borta bat jo, bersia jo eta guehia'o ono, neho'cke'e ez tsitien barne'at utzi nahi isan, ez deujs eman nahi isan jate'at. Gusieta'ic cajsatiac, jseguitiac chacur gachtos, bi arrots horiec, ez tsakiten no'at juan, noiz eta 'ê, guertatu baitsiren herriaren burian, guison eta emaste pobre hen etche aintsinian. Hen pleiniac aditsearekin, senharra athera sen leihora, her oihu eguiteco, eta haren emastia fite juan sen athiaren ide'itze'a. Hec nor si'en galde'in gabe, barne'at jsara'asi siusten eta jarra'asi su basterian; guero ekharri se'eten ejsne eta gastena sombait hec siusten gusiac. Bainan ordian Jesu-Chrichto gure jauna, - hura bera sen jondon Petri'ek'in, - chutitu sen, buria dirdirant, eta erran ze'en: "Siec pobriac siste, bainan hunac siste eta sien ausuac aberatxac eta gachtuac. Jaincoaren jujstisia eguin bedi." Be'ehala lurra ikhaatu cen, Jesus eta jondone Petri bichtatic galdu si'en (ou bien; itsali si'en) eta bi saharrac belhau'nico jarri si'en. ... Bihamoun goisian herrirat juan nahi isan sutelaïc, ser pajsatu cen jakiteco, ezt'suten ikujsi etche'ic bate'e, hen lekian basen putsu bat soina ikhujsten baita ono egungo egunian Barbazaneco herriaren eta $\mathrm{S}^{\mathrm{t}}$. Bertrand-de-Commingeco hiriaren artian.

Behar dire jende beharrac lagundu

Nota bene: ikujsi (vu). Le son js avec une voyelle est différent du son s accompagné d’une voyelle. Ceci est très important pour la bonne prononciation du basque.

\section{oOo}

Tantugou da guison sahar bat oyhanetan gordetzen dena. Saintzen'tu alhorrac eta pentziac han deujs ebatjs ez desaten. Oguiac eta belharrac picatiac di'elaic (ou bien: di'enian) hen denac naujsias beras alchatiac isan artio, Tantugou ez da batere lo'khartcen: bethi guardian dago. Haren ikujsteco beldurrac isitsen tu ohoinac. Se'ur guison huna dela, bainan basa da. Hanits gausa zakien laborari batec erraiten se'en ardura bere jsemer Tantugou dela se'urki lurreco fruitien sainsale'ic hobe'ena.

Ustaileco atjsalde bates ardi tropa'at (ou bien multso bat) alha sen mendi batian; artsaina lokhartu sen: i'atsarci'an ikhujsi sien Tantugou ihejs sohala oihane'at. Guijsa berian isan sen arrajs untxa esa'utia arsaignez selhaï pollit batian, etsana dagola harri pegna baten gainian.

Egungo egunian ere ôno Pyrenetaco sombait herritan haurrac haren beldur dira. Muthico ttipi batec edo nechcato ttipi batec bere ama'ri obeditu nahi ez tielaic, amac erten daco: Guardi'emac (et au féminin: Guardi'eman) Tantugouri oihu eguiten dake'at (au féminin: dak'enat) eta ichtantian oihanetic jinen duc (au féminin: jinen dun).

Nota bene: Dans le pays basque, pour faire peur aux enfants, on leur dit: "Mamou!". Ce qui semble dériver de "Mameluk" en basque, "Mamelouc". En effet, les Mahométans ont visité ces parages et ont dû produire sur l'esprit vulgaire une impression peu favorable.

Jean Candau, 36 ans, né à Mendive Coll. Etchart, Petecha, maire. 


\section{Barbazanen historia}

Baziren lehenago Pyrenetaco herri batean arras çahar ciren guizon eta emazte bat. Etzuten etche tipi bat, baratce bat eta behi bat baicic. Bainan zembat pobre izan citen, socoritzen ciuzten bethi hec baino pobreagoac cirenac. Egun batez lurra elurrez gorde cen eta horma ai cen harrien erdiratzeko guisan. Herrico yende guciac berotcen ciren, yaten eta edaten ari cielaic, aberats eta urrus cien. Ilunaarrian hurrundic heldu cien, bi piyayant, guelditu nahi izan ciren leku hartan, ceren eta hotz eta gose baitziren: yo zuten borta batian, guero berze batian eta berze batzutan oono: nehokee etzitin nahi izan utzi sartcera ez eta deuser yaten eman. Alderdi gucietaric casatiac, persegituac chacur coleratuz, bi estranyerec etzakiten noat youan, causitu cienian herriain basterrian, pobre cien guizon eta emazte haien [?] etchiain aitzinian. Aditzian hen plaignuac, senarra eman zen leihoan hen deitzeco eta haren emaztia youan zen fite bortain idiitzera. Her galdegin gabe nor ziren, sarrarazi eta yarrarazi ciuzten supazterrian; gero cerbitzatu zuten esnia eta zembait gazten, zuten guzia. Bainan ordian, gure Yinco Yaunac - hura zen, Yondoni Petriikin- yeiki cen, buria arras distirant eta erran zeeten: "Ziic, pobriac eta honac ziizte, ziin auzoac aberatsac eta gaichtoac dira, Yincoain yusticia ein dadiila" Ordian berian lurra icaratu cen, Yesus eta Yondoni Petri bichtatic jouan cien eta bi zaharrac belaunico erori cien.... Biharamoun goician, herrirat youan nahi izan zutenian, yakiteco ce paastu zen, etzuten icousi etcheic batre: hen ordainez: bazen hur gueldi bat St Bertrand-de-Comminges heriain eta Barbazan herriain artian causitzen dena oono egun.

Behar dira lagundu malurussac.

\section{Tantugouin historioa}

Tantugou da oihanetan gordetzen den çahar. Zaintzen tu alhorrac eta peintciac amorecatic eta deus ez dezaten ebats. Oguiac picatiac tienian edo belarra ebakia guciac barnerat sartiac izanac artio nausiaz. Tantugouc ez du batre loic eiten; iatzarriric doo bethi. Haen icousteco beldurrac atchikitzen tu ohoinac. Hona da, sinhetsazue, bainan salbaia da. Hanitz gauza zakin laborari batec erraiten zeen ardura bere semer Tantugou zela seurki lurreco fuituen zaintzaleic hobeena.

Uztaileco ilaiteco atsalde batez, artalde bat alha zen mendi baten gainian, arzaina locartu zen: iatzartzian icousi zin Tantugou çoin ies baitzoan oihanerat buruz. Ezagutia izan zen ere arzainez ordoki pollit batian, pegna batian etzana.

Eun oono, Pyrenetaco herri zombaitetan, haurrac harendaco beldur dira. Mutico ttipi batec edo nechcato batec ez diinian obeitu nahi bere amai, haurrei erraiten daco: Casumac hiri! Deitzen diat Tantugou eta berehala, yinene duc oihanetic! 


\section{Barbazaneco ichtorioa}

Baciren lehenago Pyreneetaco herri batian guiçon bat eta emazte batt arras çaharrac. Etzuten etche ttipi bat, baratze bat eta behi bat baïcic. Baïnan cembat nahi pobre içan citen, sokhorritzen ciuzten bethi hec baino pobreago cirenac. Egun batez, lurra estalia cen elhurrez eta kharroina ari cen harriac arrailatzeco guisan. Herrico yende guciac berotzen ari ciren yaten eta edaten çutelaric; aberats ciren eta uros. Ilhuntze sartzian, urrundanic heldu ciren bi bidayantec nahi ukhan çuten guelditu lekou hartan, ceren eta hotz eta gose baitzuten; yo çuten bortha batean, guero beste batean eta bertze batzuetan oraino: nihun ecin içan çuten leihorric, nihorc etzioten eman nahi içan yaterat. Orotaric khasatiac, guibeletic seguitiac chakhur oldartu batzuez, bi estranyerec etzakiten norat yohan, noiz eta ere khaüsitu baitziren herriaren bazterrean, pobre ciren guiçon haren eta emaztearen etchearen aintzinean. Heyen pleinuac entzutearequin, senharra yarri cen leihoan heyen deitzeco eta haren emaztea yuan cen fite bortharen idequitzera. Hayer galdeguin gabe nor ciren, sar araci cituzten eta jar araci beren supazterrian, guero cerbitzatu ceeten esnia eta cembait gaztaina, erran nahi baita çuten gucia. Bainan ordian Gure Yaincoa - hura cen Yondoni Petrirequin- yequi cen, burua distirant eta erran ceen: Cuec ciezte pobre bainan honac; çuen auçoac aberatsac eta gaichtoac. Yaincoaren yusticia eguin dadiela! Mement berean lurra ikharatu cen Yesus eta Yondoni Petri ezestatu ciren eta bi çaharrac erori belhaunico... Biharamoun goicean, nahi ukhan çutenean yuan herrira, yaquiteco cer pasatu içan cen, etzuten ikhusi etche bakhar bat: hayen lekhian bazen putzu bat, çoïna causitzen baita oraino egun Barbazaneco herriaren eta Yondoni Beltran Comingeco hiriaren artian. Behar dira malurusac lagundu.

\section{Tantuguren ichtorioa}

Tantugu da guiçon çahar bat oïhanetan gorderic egoitea maite duena. Beguiratzen tu landac eta pentzeac nehorc han deüsic ébats ez dezan. Oguiac ébaquiac eta belharrac pikatiac direnian, Tantugu ez da lokhartzen ogui eta belhar hec guciac nagusiac barnerat bildu arte. Haren aguertzen ikhusteco beldurrac guibelarazten ditu ohoïnac. Hona da, sinhest azue, baïnan salbaïa da. Laborari hanitz gaüça çaquien batec erraiten çuen ardura bere semeer Tantugu cela segurki lurreco fruituen çaintzaleric hoberena.

Uztailleco atsalde batez, ardi tropa bat alhatzen cen mendi baten gaïnean; artzaïna lokhartu cen; itzartziarequin ikhusi çuen Tantugu oïhaneco alderat ihes çuhela. Guisa berian eçagutia içan cen artzaïnez celhaï pollit batean etzana harri peigna baten gainean.

Oraïno egunean, Pyrénéetaco herri cembaïtetan, haürrac ikharan daüde harendaco. Noiz eta ere muthico ttipi batec edo nechcato ttipi batec ez baitu obéditu nahi bere amari, hunec erraïten daco: Güardia emac hiri! Deitzen diat Tantugu, eta ichtantian yinen duc oihanetic.

Instituteur: M. Estéot, né à Ahaxe, canton de Saint-Jean-Pied-de-Port Coll.: J. Errécart 
Baciren lehenago Pyrénéetaco herri batian guiçon bat eta emazte bat biciki çaharrac. Etçuten etche ttipi bat, baratce bat eta behi bat baicic. Bainan cembat nahi pobre ciren, laguntzen cituzten bethi berac baino pobriagouac. Egun batez, lurra elhurrez estalia cen eta harriaren arrailtceko kharroïna ari cin. Herrico yende guciac berotcen çauden yaten eta edaten ari cirelaric; aberatsac eta urosac ciren. Ilhun nabarrian, bi pidayant urrunetic heldu cirenec, nahi içan çuten lekhu hartan guelditu, hotz eta gosé baïtciren; yo çuten bortha batian, guero beste batian eta beste batçuetan oraino. Nehorkec etcitin sartcera utci nahi içan, ez eta ere deusere yatera eman. Orotaric kasaturic, seguituric chakhurrez furian, bi estranyerrek etçakiten norat yuan, noïz eta ere khausitu baitciren herri bazterrian guiçon eta emazte pobre heyen etche aitcinian. Heyen plegniac intçutiarekin senharra yarri cen leïhuan her oïhu eguiteco, eta haren emaztia yuan cen çalhu bortharen idekitcera, galdeguin gabe nor ciren; sarraaci cituzten eta yarraaci su bazterrian; guero, cerbitçatu cereten esnia eta gaztaïna cembait; cituzten guciac. Bainan ordian, Yainco-Yauna, - hura zen Yondoni Petirirekin - yeiki cen, buria argui arguia eta erran ceren: "Ciec pobriac eta hunac cizte, cien auçuac aberatsac eta gaichtuac dira. Yaincoaren yusticia eguin dadila!" Dembora berian lurra ikharatu cen Yesus eta Yondoni Petiri itçali ciren eta bi chaharrac erori ciren belhaunico... Biharamun goïcian herrirat yuan nahi içan çutelaric cer phasatu cen yakiteco, etcheric batere etçuten ikhusi: heyen plaçan laco bat cen, egun oraino khausitcen dena Barbazaneco herriaren eta Saint-Bertrand-Commingesco hiriaren artian.

\section{oOo}

Tantugou chahar bat da oyhanetan gordetcen dena. Beguiratcen tu alhorrac eta phentciac ezteçaten eta deusere ebats. Oguiac eta belharrac picaturic direlaric, denac nausiaz sarthuric içan artio. Tantugouc eztu loric batere eguiten beillatcen du bethi. Haren aguertceco beldurrac atchikitcen tu ohoïnac. Huna da, sinhets çacie, bainan basa da. Hainitz gauça çakien laborari batek erraiten ceren ardura bere semer Tantugou cela seür lurreco fruitién beguiraçaleric hoberena. Uztarilaco eguerdi ondo batez, ardi tropa bat alhatcen ari cen mendi baten gaïnian; artçaïna lokhartu cen; iratçartciarekin ikhusi cin Tantugou oïhaneco aldeat ihes çüala. Balea poillit batetaco artçainez berdin eçagutia içan cen etçanic harroca baten gaïnian.

Egun oraino, Pyrénéetaco herri cembaitetan, haurrac harendaco beldur dira. Muthico ttipi batec edo nechcato ttipi batec obeditu nahi eztilaric amari erraiten daco: "Guardia emac hiri! Tantugou deitcen diat, eta, ichtantian, oyhanetic yinen duc." 


\section{La légende de Barbazan}

Basen herri batean guison bat eta emaste bat arratz çaharrac. Ez souten etche ttipi bat, baratce bat eta behi bat baisic. Bainan, çombat nahi pobre içanic ere, socorritsen citousten pobriac. Egun batez lourra elhourrez estalia sen, karroina handia sen. Herrico yende gouciac berotcen siren yaten eta edaten hari cirelaric; aberatsac eta ourousac ciren. Ilunsian bi pidayant ourroun danic heldou çirenac guelditou nahi isan souten lekhou hortan, ceren hotz eta gose baitsiren; yo souten bortha bathi, guero bertce bati, eta bertce hainitz eri: nehorc ere ez sitouen outsi sartcerat ez eta eman yaterat. Khasatiac lekhu goucietaric, seguitiac chacourrez, bi estranyerec ez sakiten norat youan noiz eta ere heltsen baitira herriaren bourourat guison eta emaste haren etchiaren aitcinerat. Heyen oyuac aitouric, senarra heldou da leyorat oyou egiterat eta emastia badoa athiaren çabalcerat. Galdeguin gabe nor diren sar harasten eta yar harasten ditouste sou ondoan. Guero emaiten deezte sitousten gaousetaric, esnia eta gastaignac. Bainan ordian goure Yaunac - houra sen Yondone Petiriekin- choutitsen da, bouria arguitia eta erraiten dee: "Çouec pobriac eta honac siiste; souen haousoac gachtoac eta aberatsac. Yincoaren yusticia eguin dadiela". Berehala lourra ikharatou sen, Yesous eta Yondoni Petiri galdou siren eta bi çaharrac yarri siren belhaunico. Biharamoun goisian, nahi isan soutelaric herrian sarthou yakiteko çer pasatou sen, ez souten ikhousi etche bihi bat; heyen plasan, poutsou handi bat, kausitsen dena egun Barbazaneco herriaren eta Yondoni Bertrand Gommingeko hiriaren artian. Behar da lagundou malhourousa.

\section{La légende de Tantugou}

Tantugou da guiçon çahar bat oyanetan gorderic dagona. Kasou eguiten dou alhorreri eta pinceri ez desaten ebatz. Oguiac edo belharrac picatiac direlaric, denac barnerat sarthou artino, Tantugou ez dou loric eguiten; beilla da bethi. Haren ikousteko beldurrac atchikitsen ditou ohoinac. Hona da, sinhetss nesasou, bainan çalbaya. Laborari batec hainitz gautsa çakienac erraiten souen ardoura bere semeri Tantugou sela segourki lourreko fruitouen gardianoric hoberena.

Agorrilako atsalde batez, arthalde bat alhan sen mendi batean; artcaina lo sen. Iratsarciarekin ikousten dou Tantugou ihes eguiten oyanetarat. Esagoutia isan sen ere artçaines, celhay batian, etsana arroca baten gainian.

Oraino egun, goure mendietaco herri batsouetan haurrec beldour handia doute haren dako. Moutiko ttipi bat edo nechkato ttipi batec ez badoute amari obeditou nahi, amac erraiten dou: Kasou isac hiri! Oihu eguinen diat Tantugou, yinen douc oyanetik!

Instituteur: M. Elissagaray, né à Ciboure le 8 juillet 1863 Coll.: M. Chango, instituteur en retraite; Crespo, négociant; Monaco, garde forestier 


\begin{tabular}{lll}
\hline OSSĖS / ORTZAIZE & (Canton de Baïgorry) & Population: 1786 \\
\hline
\end{tabular}

\section{Barbazaneco erranfrena}

Bacen bertze orduz Pyrenetaco herri batean guiçon bat eta andre bat çoïnac baïciren arras çaharrac. Ez çuten etche ttippi bat, baratce bat eta behi bat baicen. Bainan hain pobriac içana gatic, laguntcen ciuzten bethi hec baino pobriago cirenac. Egun batez lurracen estalia elhurraz eta horma ari cen harrien arrailcecoa. Herrico yende gouciac berotcen ciren, yaten eta edaten çutelaric, ciren aberats eta uros. Ilhun nabarrian, bi bidayant ethortcen cirenac urrunetic nahi içan çuten guelditu toki hartan, ceren baitçuten hotz eta gose; yo çuten athe batean, guero bertce batean eta bertce batçuetan oraino: nehorc etciuzten nahi ukan utçi sartcerat, ez deusic eman nahi içan yaterat. Casatiac orotaric, seguitiac çakour oçar batçuez, bi arrotcec ez çakiten norat gan, noiz eta ere caüsitu baitciren herriaren bazterrean, guiçon eta andre pobre heien etchearen aintzinean. Aditcearekilan heien arrangurac, senharra eman cen leihoan heien deitceco eta haren andrea gancen laster idokitcera athearen. Galdeguin gabetaric nor ciren sarraraçi eta yarraraçi ziuzten supazterrean; guero, cerbiçatu çaioten esnea eta çenbeit gaztena, ciuzten guçiac. Bainan, orduan Gure Yainco Yauna - houracen, San Petrirekin- yeiki cen, burua oro distiratua eta erran çaioten: "Çuec çarete pobreac eta onac, çuen aüçoac dira aberatsac eta gaichtoac; Yaincoaren yusticia eguina içan dadiela!" Betbetan lurra ikharatu cen, Yesus eta San Petri itçaldu ciren eta bi çaharrac eroriciren belhaunico. Bihamun goicean, nahi ukan çutenean gan herrira, yakiteco cer pasatu cen ez çuten ikusi etcheric batere: heien tokian, bacen putzu bat çoina caüsitcen baita oraino egun Barbazaneco herriaren eta San Bertran Commingeco hiriaren artean. Lagundu behar dire yende necatuac.

\section{Tantugunen erranfrena}

Tantugucen çahar bat çoina gordetcen baitcen oihanetan. Ceincentu landac eta penceac deusic ebats ez deçaten. Oguiac pikatiac direnian edo belharra ebakia, guciac alchatuac içan artio naüsiaz. Tantugu ez da batere lokartcen; atciarra dago bethi. Haren aguerceco beldurrac atchikitcendu oihanac. Ona da, sinhetsaçoue, bainan da basa. Laborari batec, çoinac baitçakien asco gaüça erraiten çuen bere semer Tantugou cela seourki lurreco fruituen beiraçalerik hoberena. Uztailaco hilabeteco arratsalde batez ardi saldo bat çoan mendi baten gainean. Arçaina lokartu cen: iratcarcearekin ohartu cen Tantuguri çoina ihets beitçoan oihaneco alderat. Içan cen berdin eçagutia arçainez ordoki pollit batean etçana arroka baten gainean. Oraino egun Pyrenetaco çembeit herrietan, haürrac haren beldur dira. Noiz eta ere muthico ttipi batec edo nechkato ttipi batec ez peitu nahi sinhetsi bere amari, erraiten daco: Goman hiri, deitcen dut Tantugu eta berehala ginen da oihanetic.

Etcheto, 24 ans, Masparraute Coll.: Mourguiart Jean, 66 ans; Oyhénart, 49 ans, Lantabat 


\section{Barbazaneco conyera}

Baziren lehenago Pyrénêtaco herri batian guizon bat eta emazte bat arras zaharrac zirenac. Etzuten etche ttipi bat, baratze bat eta behi bat bezic. Bainan zembatnahi beharrac izan ziten, laüntzen ziuzten bethi hec baïno beharrao zirenac. Egun batez, lurra estalia zen elhurrez, eta horma ari zen harria arrailtzeko haïnian. Herrico yende guziac berotzen ari ziren, yaten eta edaten zutelaï; aberats eta uros ziren. Ilhunabarrian, urrundic heldu ziren bi piayantec nahi izan zuten guelditu lekhu hartan, hotzac eta gosiac baïtziren; yo zuten athe batian, guero bertze batian eta bertze batzuitan ere; nehoc ez zitîn sartzera utzi nahi, ez eta ere deusic yatera eman. Casatiac orotaiic, seguitiac chakhur oldartiez, bi arrotzec etzaquiten norat yuan, guerthatu zienian herriaren bazterrian, guizon eta emazte beharrac ziren hên etchiaren aïtcinian. Aitciarequin hên intzirinac, senharra athera zen leïhora hên deitzeco, eta haren emaztia yuan zen lasterca athiaîn idequitzera. Galdein gabe nor ziren, sar arazi zuzten eta yar arazi beren su bazterrian, guero, eman zêten esnia eta gaztena zembaï, zituzten guziac. Baïnan ordian, Gure-Yaüna, - hura zen yondoni Petrîquin,- chutitu cen, buria distirantic, eta erran zeen; "Zîc, beharrac eta hunac zîzte; zien aüzoac abâtsac dira eta gaïchtoac. Yincuaïn yustizia ein dailala!" Bereala lurra ikharatu zen, Yesus eta yondoni Petri bichtatic yuan ziren, eta bi zaharrac erori ziren belhaünico... Bihâmun goïzian, herrira yuan nahi izan zirenian yaquiteco zer igân zen, etzuten etcheic batê ikhusi: hên lekhian phutzu bat bazen, egun ôno khaüsitzen dena Barbazaneco herriaïn eta San-Betran-Cominyeco hiriaïn artian. Malurosac laündu behar dira.

\section{Tantugoûn conyera}

Tantugou da oïhanetan gordetzen den guizon chahar bat. Alhorrac eta phentziac zaîntzen tu, han ez dezaten deüs ebats. Oiak phicatiac direlaïc edo belharra ebaquia, oro naüsiac ereman artio Tantugouc ez du batê loric eiten; iratzarria dao bethi. Hura aguertzen ikhusteco beldurrac daüzca ohoïnac. Huna da, sinhetsazîe, baïnan salbaya da. Gaüza frango zaquîn laborari batec ardura erraiten zîn bere semer Tantugou zela seürqui lurreco fruitien zaintzale hobêna.

Uztaïlaco ilhaïteco atsalde batez, arthalde bat alha zen mendi baten gaïnian; artzaïna lokhartu zen: iratzarzian, ikhusi zîn Tantugou ihes zuala oïhaneco aldeat. Guisa berian ezaütia izan zen artzaïnez arana poillit batian, etzana harroca baten gaïnian.

Egun ôno, Pyrénêtaco zombaït herrîtan, haürrac haren beldur dira. Muthico ttipi batec edo nechcato ttipi batec eztîlaïc obeitu nahi bere amari, amac erten daco: "Beaüc hiri! (s'adressant au garçon) Beaun hiri (s'adressant à la fille) Tantugou deitzen diat ou dinat eta ichtantian yinen duc ou dun oỉhanetic! 


\section{Barbazaneco condjiera}

Baciren bertce ordus Pyreneetaco herri batean guiçon bat eta emazte bat biciki çaharrac cirenac. Etçuten etche ttipi bat, baratce bat eta behi bat baicic. Bainan, cembat nahi pobre ciren, sokhorritcen ciusten bethi berac baïno pobreago cirenac. Egun batez lurra elhurrez estalia cen, eta arician harrien erdiragarrico horma. Herrico yende guciac berotcen ciren, yaten eta edaten çutela, abarax eta urus ciren. Ilhuna sarceacoan, bi bideyant heldu cirenac urrundanic nahiçan çuten guelditu leku hartan, ceren eta hotçac eta gosseac baïtçauden; yo çuten athe batean, guero bertce batean eta bertce batçuetan oraïno; nehorc etcitian nahiçan utci sartcera eta deüs eman yatera. Cassatiac orotaric, hetxatuac tçakur ossoki samurtuez, bi arrotçec etçakiten nora yoan, noïz eta ere kaüssitu baitciren herriaren baster basterrean, guiçon eta emazte pobre hen etche aitcinian. Aditcearequi hayen aühenac, senharra eman cen leihoan hen deitceco, eta haren emaztea yoan cen laster athearen çabaltcera. Galdeguin gabe berer nor ciren, sarraraci eta yarraraci ciusten beren supasterrean; guero cerbitçatu ceeten ezne eta cembaït gaztena, hec çuten gucia. Baïnan orduan Gure Yaüna, - hura cen, Yaundoni Petrirequi, - chutitu cen burua ossoqui distiant, eta erran ceyen: Ciec cieste pobre eta hon; cien aüçoac dira abarax eta gachto. Yaïncoaren yusticia eguin dadila. Haïn sari lurra ikharatu cen, Yesus eta Yaündoni Petri ez estatu ciren eta bi çaharrac erori ciren belhaünca. Biharamun goïcean, noïz etare nahiçan baïtçuten yoan herrira yaquitera cer passatu cen etçuten ikhussi etcheric batere: heien plaçan bacen phutçu bat egun oraïno khausitcen dena Barbazaneco herriaren eta SanBertran-de-Commingeko hiriaren artean. Behar dira lagundu yende beharrac.

\section{Tantuguen gaïneco condjiera}

Tantugou da guiçon çahar bat oyhanetan gordetcen dena. Çaïntcen tu alhorrac eta pentceac amoreagatic deüs hetan ebax ezdeçaten.

Noïz etare oguiac segatiac baïtire edo belharra phicatia, nagussiac denac bildu arte Tantugou ez da batre loac hartcen; çaïn dago bethi. Hura aguertcen ikhus beldurrac atchiquitcen tu ohoïnac. Hona da, sinhexaçue, baïnan da bassa. Gaüça asko çakien laborari batec erraiten ceen maïz bere semer Tantugou cela segurki lurreco fruituen çaïntçaleric hoberena.

Uztarilaco hilabeteco axalde batez ardi multçu bat alha çagon mendi batean; artçaina loac hartu cen: iratçartceareki ikhusi çuen Tantugou ihes çoala oyhaneco alderat. Eçagutia içan cen guisa berean artçaïnez celhay pollit batean etçanic çagola phegna baten gaïnean.

Egun oraïno Pyreneetaco herri batçuetan haürec badute haren beldurra. Noïz etare mothico ttipi batec edo nechcatcha ttipi batec ez baïtu nahi obeditu bere amari, hunec erraïten dio: Cassu eguinçac (à un garcon) eguinçan (à une fille), deitcen diat (à un garçon) dinat (à une fille) Tantugou, eta datchicola yinen duc (à un garçon) dun (à une fille) oyhanetic. 
Bacen leheno Pyreneetaco herri batian guiçon bat eta emazte bat bisiki saharrac cienac. Etzouten etche ttipibat bécic, baatze bat eta behi bat. Baïnan hoïn beharrac içana gatic, chokhorritzen ciousten bethi hec bano beharro cienac. Egoun batez, lourra echtaliacen elhourrez, eta karroïn iten cien harrien arraïltzeco guijan. Herrico yende gouciac bérotcen cien yaten eta edaten çoutelaïc; habeatchac cien eta ourouch. Hilounarrian, bi pidayant hourrounic heldou cienac, nahi oukan çouten arrechtatou lekhou hartan, cendaco hotz eta goche baitcien; yo çouten bortha batian, guero beste batian eta bestebatçouetan ono: nehoc etciousten outzi nahi oukan chartzeat, ez heer deouje yateat eman nahi oukan. Khachatiac orotaic, chakhour gachtos chéguitiac, bi echtranyerec etçaquiten noat youan, noiz eta ere kaouchitou baïcien, herri bourian, beharrac cienen guiçon eta emazte heen etche ondouan. Heen plaignouac intçoutiarekilan, chenarra yarri cen leïhouan heen deïtatceco, eta haen emaztia youan cen fite bortha idokitceat. Heer galdin gabe nor cien, charraaci ciousten eta yarraaci beren chout ondoan; guero, eman ceeten ejnia eta cembait gastagna, cioustenac oro. Bainan ordian goure Yauna, - houa cen yandon Petriekin, - yekicen, bouria goucia arguitouic, eta erran ceen: "Ciec, beharra ciste eta hounac; cien aousouac abeatchac dira eta gaïchtouac. Yaincoaren yousticia ina içan daïla:" Behala lourra ikharratoucen, Yéchuch eta Yandone Petri bichtatic youan cien eta bi saharrac herrori cien belhaounico... Bihaamoun goïcian, youan nahi oukan çoutenian herrirat, yakiteco ce pachatou cen, etçouten ikhousi hetcheat ere: heen plaçan, bacen poutçou bat, egoun ono khaouchitzen dena Barbazaneco herria eta Saint-Bertrand-de Commingeco hiriaren artian. Behar da malerouchac chochtengatou.

$$
\text { oOo }
$$

Tantugou guiçon chahar bat da gordatcen baïta oïhanetan. Beïratcen tou landac eta phentziak ez deçaten yeauje ebatch. Oguiac picatou dienian, edo belharra ebakia, gouciac naouchiac chartouik içan artio, Tantugouc ez dou loïc iten ; iatzarric doo bethi. Haïn ikhouchteco beldourrac atchikitzentou ohognak. Houna da, chinetch çacie, bainan bacha da. Laboraribatek frango gaouça baçaskienak erten cien ardoura bere chemer Tantugou cela cheourki lourreco frouitien beïracaleïc obeena.

Oustail hilabeteco atchalde bates, ardi tropaat alhatcen cen mendi batian; artçaïna lokhartoucen: iatçartçia ekin, ohartoucen Tantugou echkapatzen cela oïhaneat. Eçaoutia içan cen ere artçaines, mendi arte pollit batian, etçanic arrocaaten gaïnian.

Ono egoun, Pyrenetaco herri cembaitetan, haourrac beldour dira haen. Noïs eta ere mouthico ttipi batec edo nechkato ttipi batec obeditcen nahi estienian bere amari, erraiten dako: Behaouc! ekharrazten diat Tantugou, eta, mementouan, yinen douc oïhanetic.

Oilloquy, né à Larceveau (Basses-Pyrénées) Coll.: M. Mirande. Iriberry, maire. 
Baciren bertce ordouz, Pyrénéetaco herrisca batian guison bat eta émazte bat bissiki çahartiac. Etçuten etché ttipi bat, baratcé bat eta béhi bat baïcic. Bana hoin béharrac ciélaĩc, laountcen ciouzten béthi bérac bano béharraouac. Egun batez elhourrez estalia cen lurra, et icigarrico kharroïn gaïtça ari cen. Herriko yendiac bérotcen ari cien, yatian éta édatian ; abératz éta content ciren. Ilhoun naarrian, bi arrotz ourrounetic heldu cirénac nahi oukhan çouten guelditou lékhou hartan, hotz éta gossé baïtciren ; yo çouten bortha bat guéro bertcé bat éta haïnitcétan oono : nehoc etciouzten nahi sarthou, ez déousic eman yatéa. Orotaic cassatiac, chakhur gaïtcac ondotic, bi arrotcec eztçakiten noat yuan, noiz étaére khaoussitou batciren herriaïn bazterrian, guison eta émazte béhar heen etché aintcinian. Héen pléiniac intçounik, senharra yarri cen leïhouan her oihou itéco, éta émaztia youan cen fité borthaïn idékitcea. Galdéin gabe nor cien sarraaci ciouzten éta yarraaci béren souphazterrian ; guéro éman cééten esné éta çombait gaztena, ciouztenac oro. Bana ordian, Gure Yainco Yaouna -oura cen Yondone Pétriékin - yeiki cen, bouria argui arguia, éta erran céen ; "Ciec, béharrac cizté éta hounac, cien aousouac abératz éta gaichto. Yincouaren yousticia in bédi ! Ordian bérian lourra ikharatucen, Yesus eta Yondoni Petri gordé ciren eta bi aïtasso amassouac belhaunikatou... Bihamoun goïcian youan nahi içan ciénian herrirat yakitéco cer passatu sen, etçouten etché bat éré ikhoussi ; héen lékhian bacen our gueldi bat, oonoré aourkhitcen déna Barbazaneco herriaïn éta San- Pétan-Commenyéco hiriaren artian. Béhar dira laoundou dohacabiac.

oOo

Tantugou guison çahar bat da, gordatcen déna oïhanétan. Çaïntcen tu alhorrac éta phentçiac ezdéçaten hantic déous ébatz. Noiz étaére oguiac edo belharrac phicatiac baitira, oro éréman artio naussiac, Tantugou ezta lokhartcen ; iatcarric da béthi. Houra houna da : sinhetsacié, bana bassa da. Laborari batec baïtcakin aniz gaouça érten céén béré sémer Tantugou céla séourki lourreco frouitien çaïntçaléic hobéna.

Eguerdi ondo batez ouztaillan, acienda saldo bat alha cen mendian ; artçaïna lokartou cen ; iartçatiaikin, ikhoussi cien Tantugou ihes çouéla oïhan aldéat. Berdin éçaoutia içan cen ounhaiez ordoki poïllit batian, harroca baten gaïnian etçanic.

Oonoré, Pyrénéétaco herri batçoutan, haourrac haen beldour dira. Mouthico ttipi batec édo nechcato ttipi batec, eztinian nahi obéditu béré amari, amac erraïten daco : Béhaouc to! (Béhaoun no !) Oïhou iten diat (dinat) Tantugouri ; éta béhala yinen duc (dun) oïhanétic. 
Baçuçun lehenago, Pyrenetaco herri batian, guiçonn bat eta emazte bat çoïnac arras çahartiac baïtcien. Etcicien etche ttipi bat, baatze bat eta behi bat becik. Bena, cien beçan pobre cielaik, soleitcen ciuzten bethi berac beno pobrio cienac. Egun batez, lurra cucutia cen elhurrez eta kharroña ari cen harrien arraïlceco phundian. Herrico yende guciac berotcen cien yatiaïkin eta edatiaïkin ; abeatx eta irus cien. Ilhunciaikin, urrundic heldu cien, bi bidayantec nahi ukhan çuten guelditu lekhu hartan, hotz eta gosiac baitcien ; yo çuten bortha batian, guero beste batian eta beste batçutan oono : nehoc etzuzten nahi ukhan utci sartzerat, ez eta yateat eman deuse. Ootaic kasatiac, seguitiac chakhour oldartiez, bi estranyerek etçakiten noat yuan, noïz eta ere khaüsitu baïtciren, herriaïn bazterian, guiçon eta emazte pobre cien hen etchiaren aïtcinian. Heien plentac entçytiarekin, senharra yari cen leyhuan her oïhu eguiteco, eta haïn emaztia yuan cen fite borthain idokitceat. Galde eguin gabe nor ciren, saraaci citien eta yaraaci beren suphazterian; guero cerbutchatu cen esnia eta gaztaña çombaït, çuten gucia. Bena ordian, Gure-Yauna - hurra cen yondané Pétrirekin - yeki cen, buria arguiric, eta eran cen : "Ciec pobre eta hun cizte, cien auçuac abeatx eta gachto dira. Yincuaren yusticia in dadila ! » Haïnbestenarekin, lurra ikharatu cen, yésus eta yondané Petri bichtatic yuan cien eta bi çaharak erori cien belhaunico... Biharamun goïcian, nahi ukhan çutenian yuan herrira yakiteco cer berri cen, etçuten ikhusi etcheik bate ; heien lekhian, bacen phutçu bat, çoina khausitcen baïta oonore Barbazaneco herriaren eta San Bertrand-de-Commingeseco hiriaren artian.

Behar dira malerusac sokhoritu.

\section{oOo}

Tantugou giçon çahar bat da çoin gordatcen baïta oïhanetan. Beiratcen tu landac eta phentciac han deusere ebatx ezteçaten. Oguiac phicatiac dienian eta belhara ebakia, oro nausiac bilduic izan artio. Tantugou ezta lokhartcen beillatcen du bethi. Haren aguertcen ikhousteko beldurrac eretenitzen tu ohoinac. Houna da, sinhetx azie, bena salbaya da. Laborari batec çoïnac baïtçakian gauça frango erten cen usu bere semer Tantugou seurki lurreco fruten beiraçaileic hobena cela.

Uztaillako eguerdi ondoko batez, ardi saldo bat alhacen mendi baten gaiñian, artçaïna lokhartu cen : iatçartciaïkin, ohartu cen Tantugouri ihex çouela oïhaneat. Içan cen berdin eçagutia artçañez ordoki poillit batian, etçana aroca baten gaïnian.

Oono egunn Pyrenetaco herri çombaït etan, haurrac beldur dira haindaco. Noiz eta ere mutthico ttipi batec edo nechcato ttipi batec ezpaitu nahi obeitu bere amari, eraiten daco : Khasu izac hii ! deitzen diat Tantugou, eta, behala yinen duc oỉhanetic!

Hoquina Urruty, né à Alçay (Basses-Pyrénées)

Yroumé, Bacho et Berçaìs 
Baiciren behi batez Pyreneetaco herri batean gizon bat eta emazte bat arras zahartiac cirenac. Ez zuten etche ttipi bat, baratce bat eta behi bat baicic. Bainan hain beharrac izanic ere, laguntcen cituzten bethi berac baino errumesago cirenac. Egun batez, lurra elhurrez estalia cen, eta kharroina ari cen harrien beren arrailcecua. Herrico yende guciac berotcen ciren yatean eta edatean ; aberats eta nahi bezala ciren. Ilhun cerratcian urrunetic heldu ciren bi bidayantec, nahi izan zuten toki hartan baratu, hotzac eta goseac baitciren. Yo zuten athe bat, gero bertce bat, eta bertze batzu ere ba oraino; nihorec ez citusten sartcerat utci nahi izan, ez eta yaterat ere ez ceetan deusere eman nahi ukhan. Orotaric haizatiac, chakhur borthitz batzu barkhuric gabe ondotik, bi arrotcec ez zakiten norat yoan noiz eta ere causitu baitciren herriaren bazterrian gizon eta emazte heen etchearen aitcinean. Heen dolamenac entzutearekin, senharra leihoan eman cen heen ekharrarazteco eta haren emaztea yoan cen berehala athearen idekitcerat galdegin gabe nor ciren, yar araci cituzten su bazterrean, gero eman ceeten esnia eta zombat gaztena : cituzten guciac. Bainan ordian, Jesu-Christo gure Yauna - hura cen Yondoni Petrirekin - yaiki cen, buru gucian dirdiran eta erran ceen : "Ciec beharrac eta onac cirezte. Cien hauzocoac aberatsac eta gaïchtuac dira. Yaincoaren yusticia egina izan bedi ! » Berehala lurra ikharatu cen, Yesus eta Yondoni Petri itzali ciren, eta bi zaharrac belhaunico yarri.

Biharamun goicean, herrirat yoan nahi izan zutelaric yakiteco cer gerthatu cen ez zuten batere ikhusi etcherik, heen lekian cen ur hil bat, egun khausitcen dena oraino Barbazaneco herriaren eta Bertrand-de-Comminjeco hiriaren artian. Lagundu behar dira beharretan direnac.

\section{oOo}

Tantugou da zahar bat, oihanetan gordetcen dena. Alhorrac eta pentceac begiratcen tu, ez dezaten deusere ebats. Ogiac picatiac eta belharrac ebakhiac direnean, nausiac oro sarthu artio, Tantugouc ez du loric egiten, iratzarturic dago bethi. Hura aguert beldurrac atchikitzen tu ohoinac. Ona da, sinhets zacie, bainan basa da. Laborari batec gauza hainitz zakienac erraiten cien ardura bere semer Tantugou cela segurki lurreco fruitien zeinzaleric hoberena.

Uztaileko ilhabetearen eguerdi ondo batez, ardi tropa bat alhan ari cen mendi baten gainean ; artzaina lokhartu cen ; iratzartu cenean ohartu cen Tantugouri ihes egiten ciela oihanetarat. Berdin ezagutia izan cen artzainez ordoki pollit batean, harroca baten gainean.

Egun oraino, Pyreneetaco zombait herritan, haurrac haren beldur dira. Muthico ttipi batec ez dielaric obeditu nahi bere amari, erraiten daco: Beira hiri! Deitcen diat Tantugu, eta berehala yinen duc oihanetaric?

Larroque [38, Montastruc 64; laguntzaileak: Iphar, " conducteur des Ponts et Chaussées » erran nahi baita bideetako enpleatua, et Iribarne, aita].

Larroque, 38 ans né à Montastruc (Basses Pyrenées) Coll.: Iphar conducteur des Ponts et Chaussées; Iribarne père. 


\section{Barbazaneco ichtorioa}

Baciren lehen Pirenétaco herri batian gizon bat eta emazte bat biciki zaharrac. Ez zuten etche bat, baratce bat eta behi bat becic. Bainan nahi den bezain bat hec pobre izanic, socorritzen zitusten bethi hec baino pobriago cienac. Egun batez, lurra cucutia zen elhurrez, eta kharrogna ari cen borthiski. Herrico yende guciac berotzen ari ziren yaten eta edaten zutelaric; aberats ziren eta uros. Ilhuntzian, urrunetic heldu ziren bi bidayantec nahi ukhen zuten baratu lekhu hartan zeren gose eta hotz baitziren: yo zuten bortha bat, gero beste bat eta beste zembait oraino; nehorc ere ez zusten nahi ukhen utzi sartzerat, ez eta ere deus eman yaterat. Casatiac gucietaric, segitiac chakhur eslago batzuz, bi estranjerec ez zakiten norat yuan, noiz eta ere causitu baitziren herriain puntan, gizon eta emazte pobre hayen bortharen aitzinian. Hayen pleigniac intzutiarekin, senharra eman zen leihora heyer oihu egiteco, eta haren emaztia youancen fite bortharen idekitzerat. Galdegin gabe nor ziren, sarraraci zusten eta yarrarazi hayen suphasterrian, gero cherbitchatu zayoten esnia eta zenbait gaztagna, zutena oro. Bena ordian, Gure Yauna, -hura zen San_Pierrekin- yeiki zen, buria osoki brillant, eta erran zeen: "ciec, pobriak eta hunac zieste; cien auzuac aberats dira eta gaichto. Yincuaren justicia eguina izan dadiela!" Memento berian lurra ikharatu zen, Yesus eta San_Pierre faltatu ziren eta bi chaharrac eroriziren belhaunico... Bihamun goizian, herrirat yoan nahi ukhen zutenian, yakiteco zer pasatu cen, ez zuten ikusi etcheric batere: hayen lekhian, zen phutzu bat egun oraino causitzen dena, Barbazan deitzen den herri eta St_Bernard_de_Comminges deitzen den hiriaren artian. Behar dira malerosac socorritu.

\section{Tantugouren ichtorioa}

Tantugou da chahar bat oyhanetan gordetzen dena. Beiratzen tu alhorrac eta phentziac deusere ezdezaten ebats. Noiz eta ere oguiac picatiac baitira eta belharra thaillatia, gucia khendia artean nausia ganic, Tantugou batere ez da lokharzen; beillatzen du bethi. Haren aguertceco beldurrac atchikitzen tu ohoinac. Huna da, sinhets zazie, bena salbaya da. Laborari hainitz gauza zakien batec ertenzeen ardura bere semer Tantugou zela segur lurreco fruituen beiratzaleric hoberena.

Uztaillako eguerdi ondo batez, ardi saldo bat alhatzen zen mendi baten gainian; artzaina lokhartuzen: iratzarziaikin ikhusi cien Tantugou ihes zoala oihaneco aldera. Izantzen gisa berian ezagutia artzainez baille pollit batian, etzanic harroca baten gainian.

Oraino egun, Pirenetaco zonbait herritan, haurrac beldur dira haren daco. Noiz eta ere muthico ttipi batec edo nechcato ttipi batec ez baitu obeitu nahi bere amari, erraiten daco: Guardia emac! Deitzen diat Tantugou eta, ichtantian, yinen duc oihanetik.

P. Béra, âgé de 26 ans, né à St-Jn Le Vieux Coll.: Jauréguito, Çubiat 


\section{Barbazaneco ichtoria}

Behin bazitzun Pyenetaco heri batian gizon bat eta emaste bat arras zaharrak. Ez zizien etche ñmiño bat bezik, baratze bat eta behi bat. Bena zombat nahi beharrak izanik ere, bethi laguntzen zitzien berak beno beharrago zirenak. Egun batez lurra elhurrez estalia zuzun eta kharroin bat zuzun harriak erdiratzecua. Herrico jende guziak berotzen ari zitzun, jaten eta edaten: aberatz zitzun eta uros. Ilhuntzian bi bidayant urrundik heldu zirenak nahi ukhan zizien han baratu, hotz baitziren eta gose. Jo zizien bortha bat, gero besteat eta guero beste frango: nehok ez zitzin nahi ukhan sartzerat utzi, ez eta jeus jaterat eman. Guzietarik khasatiak, chakur gaichtobatzuz aitzinian ibiliak, bi kampotiarrek ez ziakizien norat juan, noiz eta ere khausitu beitziren, herriaren burian beharrac zirelaco gizon eta emazte heen etche aitzinian. Heen intziriak intzunik senharra juan zuzun leihorat heer oyhu eyteco, eta emaztia juan zuzun fite bortharen idekitzerat. Galdegin ere gabe nor ziren, zarrarazi zitzien eta jarrarazi suphasterrian gero eman ziezien ezne eta gaztaina, zutena. Bainan ordian Jesu-Christo gure Jauna - zeren hura zuzun Jon Doni Petrirekin -chutitu zuzun, buria dena dirdira, eta erran ziezun: ziek beharrak ziezte eta hunak: zien hauzuak aberatz ditzu eta gaichto. Jincoaren justicia egin dadiela. Dembora berian lurra ikharatu zuzun, Jesus eta Petri itzali zitzun eta gure bi zaharrak belhaunicatu. Biharamunian herrirat juan nahi izan zirenian jakiteko zer gerthatu zen, ez zizien gehiago etcherik ikhusi: heen lekhian zuzun phutzu handi bat, zoina oraino ere ikhusten baitugu Barbazaneco herriaren eta San Bertran Cominjekoaren artian. Deus gabiac lagundu behar dira.

\section{Tantuguyn ichtoria}

Tantugu gizon zahar bat duzu oyhanetan gordatzen dena. Alhorrak eta phenziak beyratzentzi, ez dayn hetan ohoyntzerik izan. Ogiak phikatiak direnian eta belharrak ebakiak, nausiak guziak etchian sarthu artio, Tantuguk estzi lorik egiten: bethi ihatzarririk dioozu. Ohoynak geldirik diaudezu hura ager beldurez. Huna duzu Tantugu, sinhets zazie, bainan basa. Laborari jakintzu batek ardura erten zeen bere semier Tantugu zela seurki lurreco fruituen zainzalerik hoberena.

Uztailaco ilhabetiaren atzalde batez, ardi saldo bat alhan zioozun mendi pareta batian: artzaina lokhartu zuzun. Ihatzartzian ikhusi zizin Tantugu ihes zuela oyhanari buruz. Oro bat kabala zaintzale batzuz ezagutia izan zuzun, ordoki poillit batian harroka baten gainian etzana.

Oraino orai Pyreneetako zombait herrietan, haurrak haren beldurtzu. Mutiko batek edo neskato batec ez dienian ama sinhetsi nahi, amak erraiten diakozu: beauk! Tantuguri oihu egiten diakoat eta beahala oyhanetik jinen duk!...

Instituteur: Duvignau, 32 ans, Garos Coll: : Sallaberry Bernard et Indaburu Dominique

L 'instituteur soussigné aurait eu à cour de faire un travail plus complet, mais il s'est heurté à des difficultés qu'il a vainement tenté de vaincre. 


\section{Barbazanen ichtoriouaren tradouczionia}

Baziren bestordouz Pyrénétako herri batian gizon bat eta emazte bat biciki chaharrak zirenak. Etzouten etche gnimigno bat baïcik, baratze bat eta behi bat. Bana zombatnahi pobriak izan ziten, lagountzen zitouzten bethi hek bano pobriago zirenak. Egoun batez, lourra elhourrez koukoutia zen, eta kharroïna ari zen harriak erdiratzeko phoundian. Herriko yendiak oro berotzen ari zien, yaten eta edaten ari zielarik; aberats zien eta ouros. Ilhounaarrian, ourrounetik heldou zien bi piaïantek nahi oukhan zouten baratou lekhou hartan, zeren eta hotzak eta gosiak baïtzien; yo zuten bortha batian, gero beste batian eta oano beste batzouitan: nehok etzitien sartzea outzi nahi oukhan, ez deousere yatea eman. Orotaïc kasatiak, chakhur fourios batzouez segitiak, bi estranyerrek etzakiten norat yuan, noïz eta ere khausitu baitziren herriaen bazterrian, pobre zien gizon eta emazte heïen etche aïtzinian. Heïen plegniak intzoutian, senharra yarria zen leïhouan heïen deïtzeko, eta haren emaztia youana zen fite bortharen zabaltzea. Batere galdegin gabe nor ziren, sarrarazi zitouzten eta yarrarazi su bazterrian; gero cherbitchatu zeezten esnia eta gaztagna zombait, zitouzten gouziak. Bana ordian, GoureYauna, - houra zen, Yandani Petirirekin, - yeïki zen, - bourou gouzia distirant, eta erran zeen: "Ziek pobre eta hounak ziezte; zien aouzouak aberatsak eta gaïchtouak dira. Yaïncoaren youstizia egin dadila!" Ordou berian lourra ikharatou zen, Yesous eta Yandoni Petri ezeztatou zien eta bi chaharrak belhaounico erori zien... Biharamoun goïzian, herrirat youan nahi oukhan zoutenian, zer phasatou zen yakitera, etzouten ikhousi etcherik batere: heien lekhian bazen azekia handi bat, egungo egounian khausitzen dena ono Saint-Bertrand-de-Comingeko hiriaren eta Barbazaneko herriaren artian.

(Gaztarra)

\section{Tantougouren ichtorioua}

Tantugou da gizon chahar bat oïhanetan gordatzen dena. Begiratzen ditou landac eta phentziac ez dezaten handic deousere ebats. Ogiac moztiac direnian edo belharra ebakia, nagousiaz oro sarthiac izan artian, Tantougou ez da batere lokhartzen; bethi beilan dago. Houra agertzen ikhousteko beldourrac atchikitzen ditou ohoinac. Houna da, sinhets ezazie, bana salbaïa da. Anhitz gauza zakien laborari batec erraiten zien bere semier Tantougou segourki lourreko froutien gouardianoric hoberena zela.

Ouztaileco arratsalde batez, ardi tropa bat alha zen mendi batetan; artzaina lokhartou zen: iratzartziarekin, ikhousi zien Tantougou ihes zouhala oïhanerat. Gisa berian ezagoutia izan zen artzaïnez planada pollit batetan, etzanik harroka baten gainian.

Egoun ono, Pyrénétaco zombaït herritan, haurrak haren beldour dira. Mouthiko edo nechkato ttipi batek eztienian nahi obeditou amari, erraïten dako: khasu egik (edo egin $p$. une fille), ekharrazten diat (dinat p. une fille) Tantougou, eta ichtantian, yinen duk (dun p. une fille) oïhanetik.

Curutchet 25 ans, né à Iholdy, Basses-Pyrénées Coll.: Benta Pierres. 
Baciren lehenago Pyrénéétaco herri batian guiçon bat éta emazté bat biciki çahartiac. Etçuten etché ttipi bat, baratcé bat éta béhi bat baïci. Bana, hoïn beharrac ciélaïc, laountcen ciouzten bethi bérac bano béharragouac. Eoun batez, elhourrez estalia cen lourra, éta icigarrico kharroïn gaïtça ari cien. Herrico yendiac oro bérotcen ari ciren, yatian éta édatian; abérats éta content ciren. Ilhoun naarrian, bi arrotz ourrounéti heldou cirénac nahi oukhan çouten guelditou lékhou hartan, hotz éta gossé baitciren; yo çouten bortha bat, guéro bertcé bat éta haïnitcetan ono: nehoc etçuzten nahi sarthou, ez yéoussic éman yatéa. Ootaïc cassatiac, chakhour gaïtcak ondoti, bi arrotcek etçakiten noat youan, noiz étaré khaoussitou baïtcien herriaen bazterrian, guiçon éta émazte béhar hen etché aïntzinian. Hen pléiniac intçounic, senharra yarri cen léihouan her oïhou itéco, éta émaztia youan cen fité borthaen idékitcéa. Galdéin gabé nor cien, sarraaci çouzten éta yarraaci béren souphazterrian; guéro eman cééten esné éta cembaï gazténa, ciouzten gouciac. Bana ordian, Gouré Yinco Yauna, - houra cen, Yandoné Pétriékin, - yéiki cen, bouria argui arguia , eta erran céen: "Ciec, béharrac cizté éta hounac; cien aouçouac abérats dira éta gaïsto. Yincouaren yousticia in bédi!" Ordian bérian lourra ikharatou cen, Yésous éta Yandoné Pétri, gordé ciren éta bi aïtasso amassouac belhaounicatou... Biharamoun goïcian, youan nahi içan ciénian herriat, yakitéco cer phassatou cen, etçouten etché bat éré ikhoussi; hen lekhian bacen our gueldi bat, ono éré aourkhitcen dena Barbazaneco herriaren éta San-Pétan-Commenyéco hiriaren artian. Behar dira laoundou dohacabiac.

oOo

Tantugou guiçon çahar bat da gordatcen dena oïhanétan. Çaïntcen tou alhorrac, éta phentciac eztéçaten hanti déous ébats. Noïz étaré oguiac édo bélharra phicatiac baitira, oro éréman artio naoussiac, Tantugou ezta lokhartcen; iatçarric do bethi. Houra aguercen ikhoustéco beldourrac atchikitcen tou ohoinac. Houra ona da, sinhets acié, bana bassa da. Laborari batec, baitçakin anitz gauça, erten cen béré semer Tantugou céla séourki lourréco froutien çaïntçaléic hobéna.

Eguerdi ondo batez, ouztaillan, acienda saldo bat alha cen mendian; artçaïna lokhartou cen; iatçartciakin, ikhoussi cien Tantugou ihes çouela oïhanéco aldérat. Berdin éçaoutia içan cen ounhaiez ordoki poïllit batian, harroca baten gaïnen etçanic.

Oonoré, Pyrénéétaco herri batçoutan, haourrac haen beldour dira. Mouthico ttipi batec édo nechkato ttipi batec eztiénian nahi obéitou béré amari, amac erraïten daco: Beaouc to (Béhaoun no!). Oihou iten diat (dinat) Tantugouri éta béhala yinen duc (dun) oïhaneti!

Curutchiague, 52 ans né à Ordiarp Coll.: B ${ }^{\text {te }}$ Indart; Claire Lacroix 


\section{Barbazaneco kondera}

Bazen beste orduz Bachenabarreco menditaco herri batian guizon bat eta emazte bat biziki zaharrak zirenak. Ez zuten etche ttipigno bat baizic, baratze bat eta behi bat. Bainan zombat nahi pobre izana gatic, laguntzen zituzten bethi berac baino pobriago zirenac. Egun batez lurra elhurrez estalia zen eta harrigarrico kharrogna ari zen. Herrico yende guziac berotzen ari ziren yaten eta edaten ari zirelaic, abaratsac ziren eta uros. Ilhuna aarrian bi pasayant urrundic heldu zirenac nahi izan zuten guelditu lekhu hartan gose eta hotz zirelakotz; Jo zuten bortha batian, guero beste batian eta oraino beste batzuetan. Nehorc ez zitin sartzerat utzi nahi ukhan ez eta deus ere eman nahi izan jaterat. Orotaric casaturic, chakhur gaistoac jarraikitzen zeztelaric, bi arrotzec ez zakiten norat yuan, noiz eta ere khausitzen beitziren herriaren bazterrian pobre ziren guizon eta emazte horien bortharen aitzinian. Heyen plegniac entzutiarekin, senharra athera zen leihora heyen ekharrarazteko eta haren emaztia yuan zen fite bortharen idekitzera. Galde eguin gabe nor ziren sarrarazi ziuzten eta yarrarazi hen su bazterrian; guero eman zerezten esnia eta zembait gaztagna, ziuztenac oro. Bainan ordian Gure Jinco Jauna, - Hura zen Jondoni Petrirekin, - yeiki zen, bisaya argui arguia, eta erran zeren: "Ziec, pobriac eta hunac zizte; zien auzoac aberatsak dira eta gaistoak. Yaincoaren yusticia eguin dailala!" Hain sarri lurra ikharatu zen, Jesus eta Jondoni Petri itzali ziren, eta bi zaharrac belhaunico yarri ziren... Biharamun goician, nahi izan zutelaric herrirat yuan, yakiteco zer phasatu zen ez zuten etcheric batere ikhusi, hen lekhian bazen putzu bat causitzen dena oraino Barbazaneco herriaren eta Jondoni Beltran Cominyeco hiriaren artian. Beharretan direnac lagundu behar dute.

(Le Basque de la Basse-Navarre prononce ou tous les $u$ et $s$ tous les $z$ )

\section{Tantuguren kondera}

Tantugou duzu guizon zahar bat oyhanetan gordatzen dena. Zaintzen ditu alhorrac eta phentziak deus ebats ez dezaten han. Oguia edo belharra picatia denian, nausiac oro alchatu artio Tantuguc ez du lorik eguiten; bethi iratzarriric dago. Haren ikhusteko beldurrac atchikitzen ditu ohognac. Huna duzu eguiazki, bainan basa duzu. Anitz gauza zakien laborari batek erraiten zeren bere semer Tantugu zela segur lurreco fruitien zaintzaleric hoberena.

Uztailaco atsalde batez ardi tropa bat alhatzen ari zen mendi batian; artzaina lokhartu zen; iratzartziarekin ikhusi zin Tantugu oyhaneco alderat escapatzen. Artzainac ikhusi zuten orobat mendi arte poillit batian batian (sic) etzanic harroca baten gagnian.

Oraino egungo egunian Bachenabarreko menditaco zombait herritan haurrac beldur dira haren daco. Muthico ttipi edo nechcato ttipi batec nahi ez dinian sinhetxi bere ama erraiten daco: Casu eguizu! Oihu eguinen dacot Tantuguri eta istantian yinen duzu oyhanetic.

J. B Landé, 49 ans, né à Esquiule Coll.: Jean Inda, Maire 
Bazouzoun bestordouz Pyrénéetako herri batian guizon bat eta emazte bat biziki zaharrak zitienak. Etzizien etche tipi bat baatze bat eta behi bat bezik. Bena haïmbestetaano pobre izanikée sokhorritzen zitzien berak beno pobrio zienak. Egoun batez lourra estealia zouzoun elhourrez eta kharroin iten zizin harriak arrailtzeko phoundian. Herriko yende gouzia berotzen zouzoun yaten eta edaten zielarik, aberats eta ourosssak zitzoun. Ilhountzian, bi piayant ourrounik heldou zienak nahi oukhan zizien baratou lekhou hartan gossiak eta hotzak baïtzitien; yoiten dizié bortha batian, guïo beste batian eta beste batzutan oono; nehokée etzizun sartzea outzi nahi oukhan ez etaere yeousé yatea eman. Orotaïk kassatiak, chakour koleatiez perseguitiak bi estranyerrek etzakizien noat youan; noïz étaaré khaoussitzen baitira herriaïn estremitatean guizon eta emazte pobriak zien etchiain aïtzinïan. Heen pleintak intzoutiaïkin senharra yarri zouzoun leïhouan heen deïtzeko eta bere emaztia youan zouzoun fite borthain idokitzea. Galdingabe nortzien sarraazi zitzien eta yarraazi heen supazterrian. Guio cherbitchatou ziezin esnia eta zombait gaztagna, zitouzten gouziak. Bena ordian, Goure Jaüna -houa zouzoun yondoni Petrikin - yeïkitzen douzou, bouria distirant eta erraiten diezou: "Ziek, pobriak eta hounak zizte; zien aouzouak abeatsak eta gachtouaktzou. Yinkouaïn youztizia indailla!"Behala lourra ikharatzen douzou yésous eta yandoni Petri itzaltzentzou eta bi zaharrak erortzen belhaouniko. Bihamoun goïzian youan nahi oukhan zoutenian herrirat yakiteko zé passatouzen, etzizien ikhoussi etche bihi batée; heen plazan bazouzoun lako bat, egun St-Bertrand-de-Comminges eta Barbazan deitzen dien herrien artian khoussitzen dena. Behartzi maleoussak lagoundou.

\section{oOo}

Tantugou, oïhanetan gordatzen den zahar bat douzou. Zaïntzen tzi landak eta phentziak, ezdezaten yéouzé ébats. Noïz etaare oguiak phikatiak baïtira eta belharra ébakia, gouziak naousiaz erretiatiak izan artio Tantugou etzou batée lokhartzen bethi beillatzen dizi. Hain aguertzen ikhousteko beldourrak atchikitzentzi ohognak. Houna douzou, sinetsazou, bena salbaye douzou. Anhitz gaoüza zakien laborari batek erraïten zizin ardoura bere semier Tantugou zela seourki loureko froutien gouardano hobeena.

Uztailla hilabeteko atsalde batez ardi saldo bat ari zouzoun bazkatzen mendi baten gagnian; artzagna lokhartzen douzou: iatzartziaikin, aperzebitzendizi Tantugou ihes iten ziela oïhaneko aldeat. Berguisan ezaoutia izan zouzoun artzaïn batzouz erreka poillit batian, etzana harroka baten gagnian. Egun oono Pyrénéetako herri batzoutan haourrak haïn beldourtzou. Noïz etaare mouthiko tipi batek edo nechkato tipi batek ezpeïtou obeitou nahi bere amari, erraiten dikozou: Gouardimak hiri! Deitzen diat Tantugou eta ichtantian yieïn douk oïhanetik.

Instituteur: Chaho, 19 ans, né à Ainhice-Mongelos Coll.: Irigoin Pierre 
Bestorduz, bazitzun Pyreneetaco herri batian guizon bat eta emazte bat arras zaharrac. Etzizien etche ttipi bat, baratze bat eta behi bat baizic. Bainan, zombat nahi pobre ziren soleatzen zitzien bethi berac baino pobriaguac. Egun batez, lurra elhurrez estalia zuzun eta kharroin handia ari zuzun. Herrico jende guziac berotzen ari zitzun, jaten eta edaten zutela; aberatsac eta urosac zitzun. Ilhuntzian urrunic heldu ziren bi pidajantac nahi ukhan zizien lekhu hartan baratu, gose eta hotz baitziren; jo zizien bortha bat, guero beste bat eta beste zombait oraino: nehorc etzitzin nahi ukhan utzi sartzera, ez eta eman jatera. Orotaric urruntaraziac, chakur coleratiez perseguitiac, bi arrotzec etziakizien norat har, noiz eta ere heldu baitziren herriaren burura, guizon eta emazte pobre heen etchiaren aintzinera. Heen plegniac intzutiarekin, senharra jarri zuzun leihuan heer oihu eguiteco jin ziten eta haren emaztia juan zuzun fite heer bortharen zabaltzera. Nor ziren heer galdeguin gabe, sarrarazi zitzien eta supazterrian jar arrazi; guero eman ziezien esnia eta zombait gaztagna; etzizien besteric. Bainan ordian, Jesu-Christo gure Jauna -hura zuzun Jondoni Petrirekin- jeiki zizun buria distiatzen zacola eta errantziezun: "Ziec, pobriac eta hunac zirezte, zien auzoac aberatsac eta gaichtuac ditzu. Jaincoaren justizia eguin bedi!" Berehala lurra ikharatu zuzun, Jesus eta Jondoni Petri ezeztatu zitzun eta bi zaharrac jarri zitzun belhaunico.... Biharamun goizian, herrirat juan nahi ukhan zutenian jakiteko zer guerthatu zen, etzizien ikhusi etche bat ere; heien lekhian bazuzun mestera* bat, egun oraino khausitzen dena Barbazaneco herriaren eta Saint-Bertrand-de-Commingeco hiriaren artian. Behar dizie lagundu beharretan direnec.

\section{oOo}

Tantugou duzu guizon zahar bat oihanetan gordatzen dena. Casu eguiten dizi alhorrer eta phentzer eztezaten hantic deus ebats. Oguiac edo belharrac ebaki direnian, Tantugou etzu lokhartzen nousiac hec oro sarthu artio; bethi casu eguitin dizi. Ohoinac eztitzu menturatzen harec ikhus beldurrez. Huna duzu, sinhets azie, bainan basa duzu. Anhitz gauza zakien laborari batec erraiten zizin ardura bere semer Tantugou zela seurki lurreco fruitier so eileric hoberena.

Uztaillaco arratsalde batez, ardi saldo bat alhan ziagozun mendi baten gainian; artzaina lokhartu zuzun: iatzartziarekin ohartu zuzun Tantugou lasterca zoala ihesi oihanerat. Ezaütia izan zuzun oro bat artzainez baille poillit batian, etzana harroca baten gainian.

Egun oraino Pyreneetaco zombait herritan, haurrak haren beldur tzu. Noiz eta ere muthico ttipi batec edo nechcato ttipi batec ez baitu obeditu nahi bere amari, hunec erraiten diacozu: "Guardia emac! Oihu eguiten diacoat Tantugouri, eta jinen duc berehala oihanetic.

Instituteur: Epherre-Iriart, 32 ans né à Bussunarits-Sarasquette. 
Bazitzun lehenago Pyrenées-taho herri batian guizon bat eta emazte bat arras zaharrak zirenak. Etzizien etche ttipi bat, baratze bat eta behi bat baizik. Baïnan haïn pobre izana gatik, sokorritzen zitzien bethi hec baïno pobreago zirenak. Egun batez lurra estalia zuzun elhurrez eta karrogna ari harria arrailzeko pundian. Herriko jendiak guziak berotzen ari zitzun yanez eta edanez; aberats eta uros ziren. Ilhun nabarrian, bi pidayant, zoïnak urrunik heldu baïtziren, nahi ukhan zizien baratu leku hartan, zeren hotz eta gose baitziren; yo zizien borta batian, guero beste batian eta beste batzutan oraïno; nehok etzitzin nahi ukan sartzea ez ere deuse eman nahi yatea. Orotaik kasatiak, seguitiak chakur fuchos batzuz, bi estranyerrek etziakizien norat yuan, noiz eta kaüsitu baiziren herriko bazterrian guizon eta emazte probe ziren hen etchiain aïntzinian. Intzutiarekin hen plegniak, senarra yarri zuzun leyuan, her oyhu eguiteco, eta haïn emaztia yuan zuzun fite bortaïn idokitzera. Galdeguin gabe heyer nor ziren, sarrarazi zitzien eta yarrarazi beren supazterrian; guero zerbitchatu ziezien esnia eta zonbaï gaztagna, ziuztenak oro. Bana ordian, - Gure Yaüna- hura zuzun, Yondoni Petrirekin - chutitu zuzun buria oro diztiran, eta erran ziezun: Ziec pobriac eta hunak ziezte; zien aüzuac aberats eta gachtuac ditzu; Yinkuaren yustizia eguin dadila." Behala lurra ikaratu zuzun, Yesus eta Yondo Petri ezestatu zitzun, eta bi zaharrac belhaünicatu zitzun.

Bihamoun goïzian, noiz eta yuan nahi ukan baïtzuten herrirat, yakiteko zer pasatu zen, etzizien ikhusi etcherik batere; hen lekian bazuzun azeki bat, egun oraïno caüsitzen dena Barbazaneko herriain eta Saint-Bertrand-de-Comminges-ko hiriaïn artian. Behaïzie lagundu malhurosec.

\section{oOo}

Tantugou duzu zahar bat zoïna gordatzen baïta oyhanetan. Guardiatzen tzi alorrac eta pentziak hetan ezdezaten deus ebats. Noiz ere oguiak pikatiak baïtira edo belharrac ebakiak oro naüsiac sarrartio, Tantugou ezduzu lokhartzen batere; beillatzen dizi bethi. Haïn aguer beldurrac beiratzen ditzi ohoinac. Huna duzu, sinhets zazie, baïnan salbaya duzu. Laborari batek zoïnak baïtzakin gaüza anhitz, erraiten zizin ardura bere semer Tantugou seurki lurreko fruiten beiratzale hoberena.

Uztailako ilhaïtian eguerdi ondo batez, ardi saldoat alha zuzun mendi baten gaïnian; artzaïna lokartu zuzun: iatzartziaikin icusi zizin Tantugu zoala ihes oyhaneko aldeat. Guisa berian ezagutia izan zuzun artzaïnez ordoki poillit batian, etzana arroka baten gaïnian.

Oraïno egun, Pyrénéco tako herri batzutan, haürrac haindako beldur tuzu. Noiz ere mutiko ttipi batec edo nechkato ttipi batek ez baïtu nahi obeditu bere amari, erraïten dako: casu* eguinezac (pour le garçon) eguinezan (pour la fille) hiri! Deïtatzen diat (pour le garçon) dinat (pour la fille) Tantugou eta ichtantian yinen duc (pour le garçon) dun (pour la fille) oyhanetic.

* guardia emak (serait mieux). 
Bazitzun bertze orduz Pyrénétaco herisca batian gizon bat eta emazte bat biziki zaharac zirenac. Ez zizien etche ttipi bat beizic, baratze bat eta behi bat. Bana zembat nahi behar izan ziten laguntzen zitzien bethi hec bano beharago zirenac. Egun batez, lura estalia zuzun elhurez, eta karoïna ari zizin hariac hausteranocoan. Heriscaco jende guziac berotzen zitzun jatiarekin eta edatiarekin; aberax zitzun eta uros. Ilhuntziari, bi bidajant hurunic heldu zirenac nahi izan zitzun gelditu lekhu hartan zeren eta hotzak eta gosiak baitziren. Jo zizien bortha bat, gero bertze bat eta bertze batzu orano. Nehoc ez zitzin nahi izan utzi sartzerat, ez deusic eman jaterat. Casatiac orotaric, persegitiac chacur samoretaz bi arotzek ez ziakizien norat joan, gerthatu zirelaric heriscaren bazterian gizon eta emazte behar ziren heyen etchearen aintzinian. Entzutiarekin hen pleiniac, senhara eman zuzun leihoan heyen deitzeco eta haren emaztia joan zuzun fite bortharen zabaltcerat. Heyer galdegin gabe nor ziren, sar arazi zitzien eta jar arazi bere supazterrian; gero zerbitzatu ziezun esne eta gaztaina zembait, ziuzten guziac. Bainan ordian Gure Salbatzailiac - hura zuzun jondoni Petrirekin - chutitu zuzun buria osoki distirant, eta eran ziezun; "Ziec beharac ziezte eta onac; zien auzoac aberax dire eta gastoac. Jaincoaren justizia egina izan dadiela." Hainsari lura ikharatu zuzun, Jesus eta Jondoni Petri itzali zitzun eta zaharac eman zitzun belhaunico... Biheramun goizean, heriscarat joan nahi izan zutenean, jakiteco zer passatu zen ez zizien ikhusi batere etcheric: heyen lekhian, bazuzun ur geldi bat, oraino egun khausitzen dena Barbazaneco heriscaren eta Saint-Bertrandde-Comminges hiriaren artean. Behar dizie lagundu malurusac.

\section{oOo}

Tantugou duzu zahar bat gordatzen dena oihanetan. Zaintzen ditzi landac eta phenciac deusic ebax ez dezaten Ogiac phicatiac direnian edo belhara ebakia, guziac nausiaz bildiac izan artio, Tantugouc ez dizu batere loric egiten; iratzaria diagozu bethi. Hura agertzen ikhusteko beldurac atchikitzen ditzi ohoinac. Ona duzu, sinhexazu, bainan salbaia duzu. Laborari batec hanitz gauza zakienac eraiten zizun ardura bere semer Tantugou zela segurki lureko frutien zaintzaleric hoberena.

Uztarilaco araxalde batez arthalde bat alha ziagozun mendi batian; artzaina lokhartu zuzun; iratzartziarekin, ohartu zuzun Tantugouc ihes eguin zuela oihaneco alderat. Izan zuzun gisa berian ezagutia ordoki poilit bateco artzainetaz etzana haroca batian.

Orano egun Pyrenetaco herisca zembaitetan, haurac beldur ditzu harendaco. Mothico tipi batec edo nescato tipi batec ez duenian obeditu nahi amari, eraiten diacozu: Guardia emac! Galdegiten diat Tantugou, eta istantian jinen duc oihanetic (ou en parlant aux filles: guardia eman! Galdeginen dinat Tantugou eta istantian jinen dun oihanetic).

Prat, 46 ans, né à Lichans-Sunhare Aristéguy (Clément); Irigaray (Pierres), Souhourt (Jean) et Etchegoin (Jean) 
Bazen lehenago Pyrénées alderdico herri batian, guizon bat eta emazte bat biziki zahar zirenac. Ez zouten etche ttipi bat, baratze bat eta behi bat baizik. Baïnan, cembat nahi pobre ziren sokorritzen zousten hec beno pobriago zirenac. Egoun batez, lourra gordia zen elhourraz eta kharroina ari zen harrien haüsteco guisan. Herrico yende gouziac berrotzen ari ziren, yatez eta edatez, abératx eta ourous ziren. Ilhounaren hastian, bi bideyant, ourrountic yten zirenak, nahi izan ziren baratou lekhou hartan, hotz eta gose baitziren; yo zouten bortha batetan, guero beste batetan eta beste cembait etan oraino; nehorc ezuzten utzi nahi oukhen sartzera, ez eta ere deüsere yatera eman. Gouzietaric kasatiac, seguitiac chacour gaïtzez, bi estranyerec etzakiten norat youan, noïz ere ediren beïtziren herri basterian, gizon eta emazte pobre hen etche aitzinian. Hen plaïgnouac entzounik, senhara leïhora youran zen hen deïtzeco eta haren emaztia youran zen fite her bortharen çabaltcera. Galdeguin gabetaric nor ziren sar erazi zuzten barnera eta yar erazi sou bazterian; guero zerbitzatou zeren esne eta gaztaïna cembail, zoutien gouziac. Baïnan erdian goure Jinco Yaünac, bera zen Joundane Phetirekin, jeïki zen, bouria biziki argui eta erran zeren: Zirek pobre zizte eta houn; ziren aüzouac aberatx dire eta gachto: Yincouaren youstizia izan dadila eguina. Memento berian, lourra ikharratou zen. Jesous eta Joundane Phetri galdou ziren eta bi zaharac belhaünicatou ziren. Bihamen goïzian, herrira youran nahi izan zirenian yakiteco zer phasatou zen, ez zouten ikhousi etcheric batere; hen lekhian, bazen phoutzou handi bat, oraïno egun edireiten dena Barbazan herriaren eta Don Betan Comminges hiriaren artian. Behar dire malourousac lagoundou.

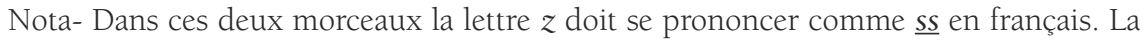
lettre $s$ doit se prononcer de manière à produire un son intermédiaire à celui de $x$ et celui de $z$, c'est-à-dire se rapprochant du ch. La lettre $y$ doit se prononcer comme un $\underline{d}$ fortement adouci, c'est-à-dire comme $\underline{d d}$. Les sons en ou au lieu d'être écrits avec un $\underline{u}$ ont été rendus par ou.

\section{oOo}

Tantugou da gizon chahar bat gordatzen dena oïhanetan. Zaintzen tou landac eta phentziac deüse ebatx estezaten. Ogiac edo belharrac phikatiac direnian, gouziac bourouzaguiac bildou estoutino Tantugou estou batere loc hartzen; bethi irrazarririk dago. Haren ikousteco beldourrac atchikitzen tou ohoïnac. Houna da, sinhets ezazie, baïnan basa da. Hanits gauza sakien laborari batec erraiten zien ardoura bere semer Tantugou, segourki, lurreko frouten zaïntzalerik hobena zela.

Oustarilako arrax aldi batez, ardi tropa bat, phasatzen zen mendi batian, artzaina loc hartou zien; irrazartzian ikousi zien Tantugou escapatzen oïhanera bourouz. Ibar poillit batian artzaïnez berdin ezagoutia izan zen etzanic zagolaric potcha baten ganian.

Egoun ere oraïno Pyrénées alderdiko herri batzutan, haurrac beldur dire harren dako. Noïz ere mothico ttipi edo neskato ttipi batek ezpitou obeditou nahi haren amari, emaïten dako: Kasou eguinan (fille), kasou eguic (garçon) deithzen dinat (fille), deithzen diat (garçon) Tantugou eta memento berian yinen doun (fille) yinen douc (garçon) oïhanetic 
MENDIVE / MENDIBE (Canton de Saint-Jean-Pied-de-Port)

\section{Barbazaneco historioua}

Baçuçun lehenoo Pyrénétaco herri batian guison bat eta emasteat biciki saharrac. Etcicien etche ttipi gnobat, baatceat eta behi bat becik. Bena hain paubre içanaatik sokorritcen citsien bethi hec beno paubrio cienac. Egun batez lurra gordia suçun elhourrez eta karroïna ari cicin harriac hausteco phundian. Herrico yende guciac berotcen aitcitsun, yanez eta edanez; aberaz eta uros citsun. Ilhunaarrian bi piayantec çoinac heldu baicien urrunetic nahi ukhan cicien baatu leku hortan; ceren gosiac eta hotsac baïcien, yo cicien borta batian, guero bestian eta besteatçutan oono; nehoke etcicien nahi ukan utci sartcea, ez uesse eman jatea. Khassatiac orotaïc; seguitiac chakur coleratiez, bi estranyerec etzakien noat yuan nois ere kaussitu baitcien herriaren basterrian, gison eta emaste paubre heen etcheco borthain antcinian. Intsutiaikin heen plegniac, senharra yarri susun leihuan heen deitceco, eta emastia yuan susun fite borthain saaltcea. Galdein gabe nor cien sarraatci citsien eta yar arassi supasterrian; guero serbitchatu ciecien esnia eta cembait gastena, siustenac oro.

Bena ordian, Gure Yauna -Yondoni Petri eta hura baitcien- chutitu cien buria oro argui arguia eta erran ciessun: "Ciek, paubriak ciste eta hounac, cien aussoac aberatsac su eta gachtouac. Yaincouain Yusticia eguina ican daila. Berehala lurra ikaratu susun, Yesus eta Yondoni Petri ecestatu citsun eta bi çaharrac erori belhauniko. Bihamun goician nahi ukan çutenian yuan herrirat, yakiteco ce passatucen ez cicien ikusi etcheat ee: han plaçan baçuçun poutçou handi, kaussitcen dena oono egun Barbazaneko herriain eta San-Bertrand-deCommingecouain artian. Behardira malurossac laundou.

Observations. Dans cette traduction tous les $e$ sont fermés, les $u$ sont mis pour ou; à part ces deux exceptions, la lecture se fait selon l'orthographe.

\section{Tantugouin historioua}

Tantugou duçu guison sahar bat gordetzen dena oïhanetan. Beiratcen ci alhorrac eta pensiac deuse ez deçaten eatz. Oguiac edo belharra pikatiac dienian, naussiac oro erretiratu artio Tantugouc etci loïc bate éguiten; beillatcen dici bethi. Haïn aguertceco beldurrac atchiquitecen tci ohoïnac. Huna oussou bena salbaïa. Laborari batec, çoinac baïtcakien hanits gautsa, erraiten cicin ardura bere semer Tantugou cela seourki lurreko fruitien beiratsaleïc hobena. Ustailako eguerdi ondoatez ardi tropaat passatcen çuçun mendiaïn gaïnian; artsaina lokartu susun: iatçarciaikin apercebitu cicin Tantugou oïhaneat ihes eguiten. Içan susun berdin eçautia asainez mendiarte pollit batian etsana harrocaten gainian.

Oono egun Pyrénéco herri çombaitetan haurrac beldurçu haïndaco. Noizee muthico edo nechkato ttipi batec ez baïtu obeditu nahi bere amari, erten diacoçu: Beaouk! Deitcen diat Tantugou eta istantian helduouc ohanetik!

Mêmes remarques pour celle-ci. Instituteur: Fourcade 


\section{Barbazaneco hitzkountcia}

Lehenago baçuçun, Pyrenetaco heriignobatian, senhar eta emaste bat biciki çaharrac cirenac. Etcicien hountasun gucitaco, etche gnimignobat, baratce gnobat eta behignobat baicik. Baïnan, çounbat pobre içanic, bethi sokhorritcen cicien berac beno pobriagoac. Egoun batez, lurra cucutia cen elhurrez eta kharroina aricen gogorki. Herrico iende guciak, berotcen ari ciren, iaten eta edaten, hek uroz ciren, ceren aberats bitciren. Ilhuntcian, bi estranier hurrunetic heldu cirenac, nahi içan çuten arrastatu herri hartan, hotz eta gose baitciren; io çuten bortha batian, gero bertce batian eta bertce frangotan. Nehorc etci usten nahi utci sarcerat, ez eta eman deüjic yaterat. Casatiac orotaïc, segitiak chakhur gaistues, bi estranierec exakiten noat ioan, gertatu cirelaïk herriaren bazterrian, giçon eta emaste chahar eta pobre heen bortha aïxinean. Inçutiaekin heren plegniac, senharra iarricen leihouan, her oihu egitéco eta emastia fite uiancen bortharen idokitcerat. Batere galde egin gabe nor ciren, iarraaci çusten eta chubasterrian iarraaci, gero cherbitchatou cioten esnia eta çumbait gastagna, hec çusten guciac. Bainan ordian JesuKristo ïaüna - houra baitcen san Petriekin,- ienki cen burua iluminatia becala, eta eran cien: Ciec, pobre eta huna cieste; cien aüçuac abarats eta gaïstuac dire. Yincoaen yusticia eguina içan daila! Mémento berian lurra ikharatu cen. Yesus eta san Pedro hurtu ciren eta bi çaharrac erori ciren belhaügniko. Bihamun goïcian, nahi içan çutelaric yoan hérirat, iakiteco cer pasatcen cen, etçuten gehio ikhusi etche bihirik: heren lekhuian bacen leicebat, çoin eta oraino egun gerthatcen beita Barbazaneko herriaen, eta san Bertrande Comingeko hirian artian. Malurosak behar dira bethi sokhorritu.

\section{oOo}

Tentagu da giçon çahar bat cucutcen dena oihanetan. Kasu egitendu lander eta penxer, amorekatic eta ez diçaten deujik ebats. Ogiac picatiac direlaic edo belhara ebakia délaic, nausiac oro sartu artiraino, Tentaguk eztu loic egiten: bethi beillatcendu. Haren ikhousteko beldurrac ohoinac begiratcentu. Huna da, sinhetzçacie, baïnan salbaia da. Gaüça frango çakien laborariatec erraiten cien ardura bere semier Tentagu cela segurki lureko fruitien beiratçaleik hoberena.

Uztailako hilabetian eguer di ondo batez, acinda tropa bat alhan çagon mendibatian, artçaina loc hartu cen: iatçarcian apercibitcen du Tentagu oihaneco alderdiraat eskapatcen. Berdin eçagutia içan cen artçain batsous celhaï poulibatian harrikotor baten gainean etçanik.

Orono egun Pyrenetaco çumbat herritan haürrec beldur dute hartaz. Muthiko tchipibatek edo nescato tchipibatek eztutelarik nahi obeditu bere amari eraiten du: Guardimac hiri! Tentaguri oihu egiten dacoat eta istantian, ienen douc oihanetic! 


\section{Barbazaneco erranpena}

Baciren lehenago Pyrénéetaco herri batian guiçon bat eta emazte bat çoinac baitciren biciki çaharrac. Ez çouten etché ttipi bat, baratcé bat eta behi bat baicic. Bainan, haïn pobre içana gatic, socorritcen citouzten bethi hec baino pobriago cirenac. Egoun batez, lourra estalia cen elhourrez, eta kharroina ari cien harriac arrailtcerainocoan. Herrico yende gouciac berotcen ciren, yanez eta edanez; aberatx ciren eta ouros. Ilhoun cerratcian, bi bidayantec, çoinac heldou baitciren ourrounic nahi oukhan çouten baratou lekhou hartan, ceren baitciren hotx eta gosé: yo çouten bortha bat, guero bertce bat, guero bertce batçou oraïno: néhoc ez citouen outci nahi oukhan sartzera, ez eta ere eman yatera. Casatiac orotaric, perseguitiac chakhour fouriosez, bi estranyerrec ez çakiten norat youan, noiz eta ere khaousitou baïtciren herriaren bazterrian, guiçon eta emazte pobre ciren, héen etchiaren aïtcinian. Intçoutiarékin héen pleïnouac, senharra eman cen leïhoan, héen deïtcéco, eta haren emaztia youan cen fite bortharen idokitzera. Galdeguin gabé nor ciren, sar araci citouzten eta yar araci beren su bazterrian; guero cerbitchatu céeten esnea eta cembaït gaztaina, çouten goucia. Bainan ordian, gouré Yaounac -houra cen yondoni Petrirekin -yeïki cen, bouroua osoki distirant, eta erran céen: "Ciec, pobriac eta hounac ciezte: cien aouzouac dira aberatxac eta gaïchtouac. Yincoaren yousticia eguin dadila!" Bat batian lourra ikharatou cen, Yesus eta yondoni Petri eceztatou ciren eta bi çaharrac erori ciren belhaounico... Biharamoun goician, youan nahi oukhan çoutelaric herrirat, yakiteco cer pasatou cen, ez çouten ikhousi etche bihiric: heyen lekhian bacen phoutzou handi bat, houra çoina khaousitcen baita oraïno egoun Barbazaneco herriaren eta Saint-Bertrand-de-Commingeco hiriaren artian. Behar dici lagoundou malourosac.

\section{Tantugouren erranpena}

Tantugou da çahar bat gordetcen dena oyhanetan. Beguiratcen ditou landac eta phentciac, deousic ez deçaten ebatx. Noïz eta ere oguiac phicatiac baitira eta belharra ebaki gouciac naousiaz erretiratiac izan artio Tantugou ez da lokhartcen; beilatcen dou bethi. Houra aguertcen ikhousteco beldourrac atchikitcen ditou ohognac. Houna douçou, sinhetx azou, baïnan salbaya douzou. Laborari batec, çoinac baïtzakin hanitz gaouça, erraiten ceen ardoura bere semer, segourki Tantugou cela lourreco frouitien beguirale hoberena.

Ouztaileco atxalde batez, ardi saldo bat alhatcen cen mendi baten gaïnian; artzaïna lokhartou cen; iratçartciarekin, ohartou cen Tantugouri ihes youaiten oyhaneco alderat. Eçagoutia içan cen guisa berian artzaïez planada poillit batian, etzana harroca baten gainian.

Oraïno egoungo egounian Pyrenéétaco çombait herritan, haourrac haren beldur dira. Noiz eta ere mouthico ttipi batec edo nechkato ttipi batec ez baitou obeditou nahi bere amari, erraiten daco: Gouardimac hiri! Deitcen diat Tantugou, eta behala yinen douc oyhanetic!

Instituteur: Estéot, 30 ans, Ahaxe-Alciette-Bascassan Coll: Hégoburu Pierre et Escostéguy Baptiste 
Bacen lehen mendi hotaco herri batian, guiçon bat eta emastequi bat biciqui çaharrac. Etçuten etche ttipibat, baratce bat eta behi bat baiciq. Baïnan, hoïn pobre içana gatic, laguntcen çusten bethi heyec baïno pobriago cirenac. Egun batez, lurra elhourrez estalia cen eta harrien erdiratceco kharroïn bat ari cen. Herrico yende guciac berotcen çauden, yaten eta edaten ari cirelaric; aberax eta ouros ciren. Ilhountcian, bi pirayant heldou cirenac ourrounic nahi içan ciren guelditu lekhou hartan, ceren hots eta gosse baitciren. Yo çuten bortha batian, guero bertce batian eta oraïno bertce batçouetan: nehore ez ciousten nahi içan outci sartcerat, ez etare deusic eman nahi içan yaterat. Khassatiac orotaric, seguitiac çakhour gaïtcez, bi estranyerec ez çaquiten norat youan, noïz etare khaussitou baitciren herriaren basterrian, guiçon eta emastequi pobre heyen etchiaren aitcinian. Aditciarequin heyen pleigniac, senharra yarri cen leyhouan, heyen deitceco eta haren emastia youan cen fite bortharen idoquitcera. Galdeguin gabe heyer nor ciren, sar araci citousten eta yar araci bere su phasterrian, guero cerbitchatou çascoten, esne eta çombait gastena, ciousten gouciac; baïnan ordian, Yainco ona - houra cen Yondoni Petrirequi- choutitou cen bouria argui arguia, eta erran cioten: « Ciec pobre cieste eta houn. Cien ausouac aberax eta gaïstouac dira? Yaincoaren yousticia eguin dadiela! » Haïnsarri lourra ikharatou cen. Yésous eta Yondoni Petri itçali ciren., eta bi çaharrac erori ciren belhaunico. Biharamoun goician, noïs etare youan nahi içan baïtciren herrirat cer passatou cen yaquiterat, ez çouten ikhoussi etcheric batre: heyen lekhian bacen hour gueldi handi bat, oraï oraïno khaussitcen dena Barbazanco herriaren eta Saint-Bertrand-de-Comminges co hiriaren artian.

Behar dira lagoundou beharrac.

oOo

Tantugou çahar bat da gordetcen dena oyhanetan. Çaintcen ditou landac eta phentciac, esdeçaten han ebàx deus ere. Oguiac phicatiac direlaric edo belharra ebaquia, naussiac denac sarthou artio, Tantugou esta loc hartcen; bethi iratçarria dago. Houra agertcen ikhous beldourrac atchiquitcen ditou ohoïnac. Houna da, sinhexa çu, baïnan salbaya da. Laborari batec çoïnac asco gauça baïtçaquien, erraïten cioten ardoura bere semeï segour Tantugou cela lourreco fruitien çaintçaleric hoberena.

Oustaileaco arraxaldi bates arthaldibat alhan cen mendi batian; artçaina loc hartou cen; iratçartcian, ohartou cen Tantugouri çoinac ihez eguiten baitcien oyhaneco alderat. Berdin eçagoutia içan cen ounhayez celhay poillit batian, etçana harroca baten gaïnian.

Oraino egoun, mendi hetaco herri batçuetan haurrac beldour dira harendaco. Noïs etare mouthico ttipi batec edo neichcato ttipi batec ezbaïtou obéditou nahi bere amari, erraïten daco: Cassou eguinçac! Deitcen diat Tantugou, eta berehala heldou douc oyhanétic!

Instituteur: A. Çarçabal (51 ans, né à Chéraute près Mauléon. Coll.: Harguindeguy, Minondo) 


\section{Barbazaneco hitzcountcia}

Lehenago baçuçun Pyrenetaco herri batian gizon eta emazte bat biciki zaharrac zirenac. Etçouten huntarsun gucitaco etche ñimiñobat baratceñobat eta behiñobat baicic. Bainan çombat pobre içanik ere bethi socorritcen çuten berac baino pobriagoac. Egun batez lurra cucutia cen elhurrez eta kharroina ari cen gohorki. Herrico jende guciac berotcen ari ziren jaten eta edaten; uros ziren, abarats baitciren.

Ilhuntcian bi estrayer urrunetic heldu zirenac nahi izan çuten baratu herri hartan, hotz eta gose baitciren; jo çuten bortha batian; gero bertce batian, eta bertce frangotan: nehorc etciuzten utci nahi sartcerat ez eta eman deusic jaterat. Casatiac orotaric, segitiac chacur gaistuez, bi estranjerrek, etzakiten norat joan, gertatu zirelaric herriaren puntan gizon eta emazte pobre haren bortharen aitcinian. Inçutiarekin heren pleiñiac senharra jarri cen leihoan, heien deitceco eta emaztia fite juan cen bortharen idokitcerat. Batere galdegin gabe norc ziren, sarrarazi zouzten eta subasterrian jarrarazi; gero serbitsatu cioten esnia eta zombait gastaina hec zuzten guciac. Bainan, ordian, Jesu-Kristo Jauna -hura baitcen, yondoni Petrirekin- jeiki cen burua illuminatia, eta erran cion: "Ziek pobriac ziezte eta hunac, zien auzuak abarats eta gaistuak dire. Jaincoaren justicia egina izan daila!” Memento berian lurra ikharatu cen, Jesus eta Yondoni Petri itzali ziren, eta bi zaharrac eroric ziren belhaunico. Biharamun goician, nahi izan zutelarik juan herrirat, jakiteco zer pasatu cen etzuten gehio ikhusi etcheric batere, heren lekhuan bacen leicebat çoin eta oraino egun gerthatcen baita Barbazaneco herriaren eta san Betrande Cominyeco hiriaren artian.

Malurosac behar dira bethi sokhorritu.

- $j$ se prononce $i$

- $z$ se prononce $s$

- $\tilde{n}$ se prononce gn

- $s$ entre $s$ et $c h$

- g toujours gue

\section{Tantuguren hitzcountzia}

Tantugu da gizon zahar bat gordetcen dena oihanetan. Khasu egiten du lander eta phencer amoregatic eta ez dezaten deus ebats. Ogiac picatiac direlaric edo belharra ephaitia delaric, nausiac oro khendu artio, Tantuguc ezdu loric egiten; beti beillatcen du. Haren ikhusteco beldurrac ohoinac begiratcen du. Huna da, sinhets zazu, bainan salbaya da. Gauza frango çakien laboraribatek erraiten zien ardura bere semer Tantugu cela segurki lurreco fruitien begiratçale hoberena. Ustaileco hilabetiaren eguerdi ondo batez, azinda tropa bat alhan çagon 
mendibatian; artsaina lokhartu cen; iatçartcian, apercebitcen du Tantugu oihaneco alderdirat escapatcen. Berdin eçagutia içan cen artsain batsus celhai lekhu pollit batian harroca baten gainean etzanic.

Orono egun, Pyrenetaco çombait herritan haurec beldur dute hartaz. Muthico ttipi batek edo nescato ttipibatek ezdutelaric nahi obeditu bere amari erraiten de: Guardimac hiri! Tantaguri oihu egiten dacoat eta, istantian jinenduc oïhanetic.

Instituteur: Casemajor, 32 ans, Osserain Coll.: Barneix 
Bazien lehenago Pyrénées, mendien ondoan guizon bat eta emaztebat biziki saharrak. Etzuten etche ttipi bat baïzik, baratce bat eta behi bat. Bainan sembat nekhian izanikere bethi arrapatzen duten berak beno beharragoy ongui eguitea. Egun batez lurra guzia estaliazen elhurrez eta hormac harriak pikatzen zituen. Herriko jente guziac berotzen ariziren yathiarekin eta edatiarekin, aberats cirén eta ouros. Ilhunariburuz bi bidayant urrunetic heldu cienak nahi zuten guelditu lekhu hartan, ceren eta goze eta egarri baitzuten. Yozuten borta bati eta bertze zombaiti: Nehorek etzuten nahi izan zabaldu. Urrundiak guzietarik, chakhur gaïstho batzuez zeguituak bi guizon estrangerhec etzakiten zer eguin, norat juan guertathu zirenian herriaren akabançan hango bi jente guizon bat eta emaste bat ontza nekatiak zirenac. Heren plaintac inzunik, zenharra yarrizen leyhuan heren deizeko. Borta zabaldu eta, eman gastaïna zoumbaït eta gobor ezne bedera. Baïnan ordian Yésus-Kristo, hourazen yondoni Petirikin yekhitzen buria argui arguia eta erraten dei: Ziek praübe zieste eta honak; zien aïzuak aberatz eta gaïzto. Jinkoäin justizia eguina izan daïn! Ordain berian lurra ikharatuzen. Yesus eta Pierres essestatuziren eta bi zaharrac eroriziren belhaüniko. Bihamounian nahi oukhan zutenian juan herrirat yakiteko zer igaitentzen etzuten ikhouzi etcherik batere. Heren ordetan bazen our ondo handibat zoïna guertatzen baïta Barbazaneco eta St Vindent de Commingeko hiriaren artéan. Behar da maléruzer ongui eguin

oOo

Tantugou da zahar bat koukoutzen dena oïhanetan. Beïratzentou elguiac eta pentziak amoréakatik eztezaten deüzerik ebatz. Oguiak pikatiak tirenian edo belharra ephaitia guziac naüziak ereman artio, eztu batere loïk iten; bethi guardan da. Haïn beldurrak atchikitzentiztu ohoïnak. Hona da, zinhetz zazie, baïna zalbaiiada. Laborari zaban batek erraitentzuen uzu bere zemer Tantugou zela zeürki lurraïn fruten guarda hobena.

Atzalde batez uztaileko hilabetian azinda molchoat bazkatzen arizen borteatian: arzaina lokartuzen; yatzarziarekin, aperzeitzendu Tantugou ezpakatzen zelaik oỉhaneat. Ezaütia izantzen ere arzaïnetaïk, arrokaten gaïnian etzanik zelarik leku poïlit batean.

Egungo egunetan oïno, Pyrénées herri zoïnbatian, haürec haïndako beldur dute. Motiko ttipi batek edo nezkatoche ttipi batek eztienian haïn amari obéditu, bere amak erraïten dako: "Kasu eguinzak: deitzen diat Tantugou eta behala yen douk oyhanetik.

Simon Sabre, 21 ans, né à Saint-Palais 


\section{Barbazanen historioa}

Baziren lehenago gure borthu ondoco herri batean guizon bat eta emastequi bat ceinac hainitz adinduac baitziren. Heyen ontazun guziac ciren etche ttipi bat bere baratziarequin eta behi bat. Bainan zombat ere beharra baïziren zokorritzen zusten bethi berac baïno beharragoac zirenac.

Egun batian, lurra helhurraz estalia zen, horma belza ari. Herrico yende guciac berotzen ari ziren yatearequin eta edatearequin; aberatz eta iruz ziren. Ilhuntziarekin bi bidayant urrunetic heldu zirenac nahi oukhan zuten guelditu herri hountan, ceren hostuac eta gozetiak baitziren. Yo zuten bortha batian, guero bertze batian eta bertze ainhitzetan oraino.

Nehorc ez cituen zartzera utzi nahi ukhan beren etchetara es eman deus yatera. Kazatuac guzietaric, chakhur gaiz guziez hertzatuac, bi bidayant heyec ez zakiten norat yuan, noiz eta ere khauzitu baitziren herriaren basterrian: guizon eta emaste behar heyen etchearen aitzinean. Kampoco estranyer heyen hegadorac entzutiarekin, senharra yelgui zen leihorat heyer oihu eguiteco eta emastea yuan fite bortaren zabaltzera. Galdeguin gabe nor ziren zar arazi zitusten eta yar arazi zupasterrean, guero offfreitu ceitesten eznia eta zembait gastaina hec zituzten guziac. Bainan orduan (Jainco) Gure Yauna (ceren hura baitzen Yondoni Petrirekin) yeiki zen burua arguiz distiratzeco zakolaric eta erran cioten senhar emazte borondatez bethe heyer: "Zuec beharrac zaitezte eta onac: zuen aizoac aberatzac eta gaichtoac dira. Yaincoaren yusticia eguin dadila: "Memento berian lurra ikharatu zen, Yainco Yauna eta Yondoni Petri itzuldu ziren eta senhar eta emazte zahar hec belhaunico erori ziren. Biharamun goizian, yuan nahi izan zuten herrirat yaquitera zer pazatu zen ez zuten ikhuzi etche bat: heyen lekhian itzazo ttipi baten pareco hur bat, hur bat zoina kausitzen baita oraino egun Barbazan eta Sen Bertrand de Comminges deitzen diren herri eta hiriaren artean.

Behar dira beharrac lagundu.

\section{Tantugoren historioa}

Tantugou da guizon zahar bat oyhanetan gordatzen dena. Beguiratzen ditu elgueac eta alhorrac eta pentzeac amoreagatic eta ez dezaten deuz han ebatz. Oguiac ebakiac direnian edo belharra lurrean etzanac, Tantugou ezda lokhartzen nauziac oro barnera zar artean; bethi guardian dago. Haren aguertzearen beldurra baratzen ditu ohoinac. Ona da Tantugou, sinhetz ezazue, bainan baza da.

Laborari batec hainitz gauza zakienac erraiten zuen bere zemer segurtki Tantugou zela lurreco fruiten beguirazale hoberena. Arratzalde batez, ustaicaco hilabetean, ardi tropa bat alha zen mendiaren gainean: artzaina lokhartu zen; iratzartzearequin, ikhuzi zuen Tantugou ihez eguiten oyhanetarat. Ezagoutua izan zen berdin bertce artzain batzuez, etzanic errephera baten harroca baten gainean. Egun oraino gure borthu basterretaco herri zombaitetan, haurrac haren beldur dire. Noiz eta ere mutiko ttipi bat edo neskatcha ume batec ez baitute sinhetzi 
nahi beren amac erraten dakote: "Guardiac emac hiri!... Oyhu eguinen dakoat Tantugou eta memento berian ethorriko duc oyhanetic.

*Dans le pays Basque le nom de Tantugou est tout à fait inconnu. Les mères pour menacer les petits enfants leur disent toujours: "Yaun Guili Barbaü, yan zac haur hau: Bihar ala gaur?... gaur, gaur". Monsieur Guillaume le monstre, mange cet enfant. Demain ou ce soir?... Ce soir...Ce soir.

Instituteur: Longy, 22 ans, né à Amendeuix (Basses-Pyrénées). 
Bacen bestordus Pyreneetaco herri batetan guiçoun bat eta emaste bat çouin beitciren biciqui çahar. Ez çuten etche ttipi bat baïcic, baratce bat eta behi bat. Bena hain pobriac içana gatic, sokorritcen çusten bethi houac beno pobriago cienac. Egun batez lurra cucutia cen elhurrez eta kharroigna ari cen harrien arrailtceco phundian. Herrico jende guciac berotcen ciren yaten eta edaten cielaric; aberax ciren eta irous. Ilhuntcian bi ibilçale, hurrundanic heldu cirenac, nahi içan ciren baratu lekhu hartan ceren beitciren hots eta gosetiac; yo çuten bortha batetan, guero beste batetan eta beste batçutan orano: nihorc ez citien utci sartcera ez hayer eman deusee yatera. Cassaturic orotaric, seguituric çacur gaitcez, bi campotiarrec ez çaquiten nourat yuan, edien cielaric herriaren burian, pobriac ciren guiçon haren eta emaste haren etchiaren aïtcinian. Entçutiarequin hayen couïntac, senharra yarri cen leihouan hayen deitceco eta haren emastia yuan cen fite bortharen çabaltcera. Hayer galdeguin gabe nor ciren, sar eraci ciusten eta yar eraci beren suphasterrian, guero cerbutchatu ceyen esne eta gastagna çombait, çuten gucia. Bena ordian, Gure-Jincoua- houa cen San Pierrarequi- chutitu cen buria argui-arguia eta erran ceen: "Ciec pobriac cieste eta hounac; cien aiçouac aberaxac dira eta gachtouac. Jincouaren justicia eguin bedi!” Hain sarri, lurra ikharatu cen, Jesus eta San Pierra galdu ciren eta bi çaharrac erori ciren belhaunico. Bihamun goïcian, yuan nahi içan cielaric herrirat, yaquiteco cer phasatu cen, ez çuten icusi etcheric batere: hayen plaçan bacen phutçu bat edireïten dena orano, egun, Barbazaneco herriaren eta Saint-Bertrand de Cominges hiriaren artian.

Behar dira laguntu malerousac.

\section{oOo}

Tantugou guiçoun çahar bat oïhanetan gordatcen dena. Çaintcen tu elguiac eta phintciac ezteçaten han deusere ebax. Nois eta oguiac phicaturic beitira eta belharra ebaquia, oro naüsiac bildu artino, Tantugouc estu loric eguiten batere; iratçarriric da bethi. Haren aguertcen icusteco beldurrac atchiquitcen tu ohognac. Houna da, sinhex çaçu, bena salbaya da. Laborari batec, çougnec beitçaquian hanichco gauça erraïten cian usu bere semer Tantugou cela segurqui lurreco fruitien çaintcaleric hobena.

Ustailla hilabeteco arastiri batez, ardi saldo bat alhan ari cen mendi batetan; artçagna loc hartu cen; iratçarciarequin icusi cian Tantugou escapatcen oyhanerat. Eçaguturic içan cen orobat artçagnes ibar poillit batetan, etçanic botche baten gagnen.

Orano egun Pyreneetaco herri çounbaitetan, haurrec badicie haren beldurra. Nois eta mutico ttipi batec edo nescatoche ttipibatec espeitu nahi obeitu bere amari, erraiten dero: Abisa hadi! Deitcen diat Tantugou, eta, behala yinen duc oyhanetic.

Instituteur: Constantin, 39 ans, nè à Lahonce près Bayonne 


\section{Babazanéco Erran çaharra}

Bacien besté orduz Pyrinioétaco herri ttipi batian guiçon bat éta émazté bat arras çahartiac. Etçuten etchégno bat, baratcé bat éta béhi bat bécic. Béna, haïn béharrac içana gatic, sokhorritcen çuzten béthi bérac béno béharragouac. Egun batez, lurra elhurrez coucoutia cen, éta kharrougna ari cen harriac erdiaraztéaïnocoan. Herrico yendé guciac béotcen ari cien yatiaïkin éta édatiaikin: abératx éta irous cién. Ilhuncian, bi biayant urrunétic yinac nahi ukhan çuten lékhu hartan baatu, hotz éta gosé ciélacotz; yo çuten bortha bati, guéro besté bati éta besté batçuer oono: nihouc etçuzten sartcéa utci nahi ukhan, ez éta éré deüsé éman yatéa. Cassatiac aldé guciétaïc, chakhur gachto batçuez séguitiac, bi arrotcec etçakiten noat youan, horra herriaren burura heldiac non guerthatcen dien, guiçon éta émazte béhar héin etché aïtcinian. Entçutiaïkin héin plagniac, senharra éman cen leïhouan biayanteï oïhu éguitéco, éta émastia youan laster borthaïn çaaltcéa. Héyer galdéguin gabé nour cien, sarraraci çuzten éta yarraraci béren supasterrian; guéro, cherbitchatu céiten esnia éta çombéit gaztagna, çuténa oro. Béna ordian, (Yésu-Cristo) Goure Yauna - houra cen, Youandoni Pétrirékin- chutitu cen, buria dirdiratcen cioéla, éta erran céïn: "Ciec, béharrac éta hunac cizte, cién aïçouac bada abérax éta gachto. Yincouain yusticia éguin daila!” Erran béçain laster, lurra ikharatu cen, Yésus éta Youandoni Pétri bichtatic galdu cien éta bi çaharrac érori cien belhaouniko... Bihamoun goïcian, nahi ukhan çuténian herrirat yuan, cer igan cen yakitéa, etçuten ikhusi etchéïc batéré: héyen lékhian, bacen ur gueldi handi bat, égun oono ikhusten déna Barbazanéco herriain éta San-Bertran-Commingesseko hiriaïn artian. Behar dira sokhorritu yendé béharrac.

\section{Tantugouen Erran çaharra}

Tantugou da guiçon çahar bat oyhénétan gordetcen déna. (Ouhougnétaïc) Béatcen tu elguiac éta phintciac han ezdéçaten deüse ébatx. Oguiac moztiac diénian édo belharrac ébakiac, naoussiac oro sarthu artio, Tantugouc ez du baté loìc éguiten; ernéric dago béthi. Haïn beldurrac urrunduric atchikitcen tu ouhougnac. Houna da, sinhex çaçué, béna bassa. Anhitz gauça çakiéna laborari batec ardura erraiten cien béré semer Tantugou céla ségurki lurréko frutien béïraçaléïc hobéna.

Uztailllako axaldé batez, ardi molcho bat alha cen mendi batian; arçagna lokhartu cen: iatçartciaikin ikhussi cien Tantugou ihési çoala oyhénari buruz. Guissa bérian beste arçain batçuec éré éçagutu çuten mendiarté pouillitgno batian, etçana harroka baten gagnan.

Egun oono, Pyrinioétaco herri çombéitétan, haourrac beldur dira haïndaco. Mouthicogno batec édo nechcatuchégno batec ez diénian sinhetsi nahi béré amari, ordian erraïten daco: Gomac hiri! Oïhu éguiten dacoat Tantugouri éta béhala, yéïnduc oyhénétic!

Notes sur quelques sons particuliers à la Langue Basque

$$
e=\text { toujours } e ́
$$


$h=$ toujours aspiré

$k h=$ avec un son guttural

$l l=$ on les mouille

$p h=$ appuyer sur le $p h$ aspiré

th $=$ aspirer le $h$

$t t=$ on les mouille

$s=$ mais d'une manière particulière à la Langue Basque, qu'on ne peut expliquer et qui ne s'apprend que par l'usage, encore l'étranger n'y arrive-t-il pas toujours.

$z=c ̧$ mais avec un petit sifflement.

Instituteur: Menvielle (Pierre), âgé de 48 ans, né à Montestrucq [=Ozenx-Montestrucq] Coll.: Bohotéguy (Simon). Garat (Pierre) 
Baçuçun bestordous, Pyrénées deitcen dien borthu oundouetaco herri batetan guiçounbat eta emasteat biciqui çaharrac. Etcicien etchéchipignoat, baatciat eta behignoat becic. Bena çoumbat nahi praoube içanaatic, halaere laguntcen citcien berac beno praoubiagouac. Egun batez, lurra coucoutuic çuçun elhurrez eta kharrougna ari çuçun biciqui azkarki. Herrico yende guciac ai citçoun yaten eta edaten berotciaïkin: aberassac eta iroussac citçun. Ulhuntcian, bi bidayant urhunic heldu cienac baâtu içan citçun lekhu hartan, céen eta hotz eta gossé bitcien. Bortha batetan yoeitin tcie, guero beste batetan eta fountsian beste hanitsetan; bena nihourq etcicin sarceat utci nahi oukhan ez yateat eman. Oôtaric kassatuic hersatuic, persituic chakhur maleciatiez bi estranyerrec etzaquicien nouat youan, nouiz eta ere errecountratu beitcien herri basterrian, erran delaco guiçoun eta émasté praoubé heyen etchiaen aitcinian. Heyen plaigniac untçunex, senharra yelkhi çuçun leïhoat heyer oïhu iteco eta emaztia youan borthaïn çaalcea. Galda in gabé nour cien, sarraasten tcié éta yarraasten supasterrian, guero cherbuchatcen deicié eznia eta gastagna çoumbeit, çutena oro. Bena ordian Yesu Christo goure yaouna (Youandene Petrie ta houra citçun) -houa çuçun Youandene Petriekin, yekiten tçu, buria argui arguia eta erraiten deiçu: ciec praoubiac eta hounac cisté: cien aissouac abeaxac eta gaichtouactçu. Yincoain yusticia içan daïlala inic. Hambestenaïquilan lurra ikaatu çuçun Yésus eta Youanden Petri galdu citçun eta bi çaharrac belhaïco erori. Bihamon goïcian, youan nahi içan cienian herrirat, yaquiteco cer igan cen, etcicien guehiago etcheïc ikhoussi, heyen orde baçuçun our barna eta gueldi bat çouign ikousten bita oano egun Barbazaneco herriaren eta Seen Bertran Commenyeco hiriaren artian. Behartcié socchorritu maleousec.

\section{oOo}

Tantugou çu guiçon çahar bat oïhenetan gordatcen dena. Elguiac eta phinciac beiratcen tci esteçaten yoouséré ébats. Oguiac ébaquiac dienian edo belharra phïcatia oro naoussiac idequic artian, Tantugouc etci loïc batere iten, bethi goueïtian daouçu. Hain ikousteco beldurrac urhuntcen tci ouhougnac. Hounaouçu, segur içan cité, bena baï salbayia eré. Biciqui gaouça çakien laborari batec erraiten cicin ussu bere semer Tantugou cela seurki lurreco frutien beïraçaleic hobena. Ustaillaco atsaldé batez ardi tropabat alha çuçun mindi batian; arçagna lokhartu çuçun: iraçartciaïkin ikousten tci Tantugou oïhenetalat ihez iten. Beste arçain batçuc eré eçaoutu cicien, plana tchipi batetan etçanic arroka baten gagnan. Egungo egunian eré, bortu oundoco herri batçutan haourrac beldur tçu haindaco mouthico tchipi edo nechcatoché chipi batec espadu ama sinhexi nahi, amac erraiten dacoçu gomac! Edo gomaneçan oïhu inen diat edo inendinat Tantugouri eta behala yien douc edo dun oihenetaïc.

Instituteur: Goyheneix, âgé de 40 ans, né à Arbouet Coll.: Goyheneix père 
Bacien lehenago Pirene-etaco herribatetan guiçon eta emastebat hanich çaharrac. Etcien etche chipibat, baratcebat eta behibat baïcic. Bena çoumbat nahi praübe cien, sokhorritcen çutien berac beno praubiago cienac. Egun batez lurra elhurrez gordeic cen eta kharrouk erdiatcen cin. Herrico gentic oro berotcen ari ciren, jaten eta edaten cielarik; aberatx eta irous ciren. Ulhuntcin hurrunti jiten cien bi bidejant nahiçan ciren baatu lekhu hartan, ceren hots eta gosse beitciren; jo cien bortabat, guero bestebat, guero besteik ere orano: ihourk ere etçutin nahi ukhen utci sartcera ez etare deüs eman jatera. Baster orotaric akassaturic, chakur gaïtcez seguituric, bi estrangeec etzakien nouat joun nouiz etare ediren beitciren heriaen burin, praübe cien guiçon eta hayen etchiaen aïtcinin. Hayen plinten entçutiarekin, senhara jarri cen leyhoun hen deitceco eta haren emastia fite jouan cen borthaen çabaltcea. Galthatu gabe nour cien, sareaci çutin eta jar-aci beren suthondoun; guero eman jatera ezne eta gastagna, hec ciena oro. Bena ordin, Goure Jauna -houra cen joundane Phetirieki -jeiki cen buria argui arguia eta erran ceren: "Ciec praübe eta houn cidie, cien aiç̧ouk aberatx eta gaichto dira. Jincouaren justicia eguin dadila!" Haïn sarri lurra ikharratu cen, Jesus eta joundane Phetiri galdu ciren eta bi çaharrac errori cien belharico... Bihamen goïçan, herrilat joun nahiçan cienin cer igaran cen jakiteko, etcien ikhoussi etcheric batere: hayen lekhin bacen losco handibat, egun oano edieiten dena Barbazaneko herriaren eta Sain. Bertrand-de-Comminges-eko herriaren artian. Behardia lagundu malerousak.

\section{Historia çahar bat (Tantugou)}

Tantugou da guiçon çahar bat, çoin gordatcen beita oïhanetan. Beguiatcen tu elguiac eta penciac amourecatic eta deusere estecen ebatx. Ogik mousturic eta belharrak ebakiric dienin, naüssiac oro bil artino, Tantugou esta lotcen batere, atçarriric dago bethi. Harren aguertcen ikousteco loxak hurrun etchekitcen tu ouhouinak. Houn da, sinhetx eçacie, bena salbaje da. Hanichko gaïça çakin laboraribatec erraiten cin ussu bere semer Tantugou cela segurki lurreko fruten beguirariric hobena. Ustarilaco aristiri batez, ardi saldobat alhan ari cen bortin; artçaina lothu cen: iratçartcin, ikhoussi cin Tantugou escahatcen oïhanilat burus. Bardin eçaguturic içan cen abere bequiraçale batçuz nava ejer batetan, arroca batetan gagnen etçanic. Egun ere oano, Pireneetako herri çombaïtetan, haürrac loxa dira harentaco. Nouiz ere mouthico edo neskatchouna chipi batec espeitu nahi bere ama sinhexi, eraiten deo: Abisa hadi! Tantugou deitzen diat (edo dinat) eta behala ginen duc (edo dun) oïhanetic.

Instituteur: Carriquiry Alexis, âgé de 44 ans, né à Haux (Basses-Pyrénées) Archelaco Pierre et Bordat Dominique 
Bazen beste ordouz Pyrénéko herri batian guizon eta emazteki bat biziki zahar zirenak. Etzouten etche ttipibat beyzik, baratzebat eta behibat. Zoumbanahi paubriak ziren sokhoritzen zouzten bethi berak bayno paubriago zirenak. Egoun batez, lourra estaliazen elhourrez eta kharroina arizen harriaren erdiratzeko guisan. Herriko yende gouziak berotzen ari ziren, yatian eta edatiarekin aberatz eta irouz ziren. Ilhountzian bi bidayant ourrounetik heldou zirenak nahi oukhanzouten baratou lekhou hartan zeren eta gose eta egarri beytziren; yozouten bortha bat, guero beste bat eta beste batian oraino, nihork etzouten nahi oukhan sartzerat outzi ez eta ere deouz yaterat nahi oukhan eman. Orotarik kasatourik, perseguitourik chakour gachtouez, bi estranyerrek etzakiten norat youan, noiz ere khausitou beytziren herriaren basterrian, guizon eta emazte paubre heyen etchiaren aytzinean. Inzoutiarekin heyen pleiniak, senharra youanzen leyhora heyer oyhou eguiteco eta haren emastia youantzen fite bortharen zabalzera. Heyer galdeguin gabe nor ziren, sar arazi eta yar arazi ziouzten beren souphizguian, guero zerboutchatou zioten eznia eta gazteyna zounbeyt, ziousten gouzia. Baynan ordouan, Goure Yaüna -hourazen, zan Pierrekin- choutitouzen, bouria distiratourik, eta erran zayoten: "Ziek, paubre zieste eta houn; zien ayzoak aberats dire eta gaychto, Jaincoaren yustizia eguinik izan dadila!" Hainbeztenarekin lourra ikharatzen da, Yesous eta san Pierre youan ziren eta bi zaharrak erori ziren belhaunika.... Biharamoun goyzian, nahi izanzirenian youan herrira, yakiteko zer phasatouzen, etziouten ikhouzi etcherik batere; heyen orde, hour gueldibat zen, zouynere khausitzen beyta orayno egoun Barbazaneco herriaren eta San BertranCommingeneko hiriaren artean. Behardire sokhorritou malour dounak.

\section{oOo}

Tantugou guizon zaharbat da oyhanetan gordatzen dena. Beyratzen ditou alhorrak eta penziak amorekatik eta deousik han ebatz ez dezaten. Noiz ere oguiak phikatiak beytire edo belharrak ebakiak, nauziak gauzak oro zar ararteka, Tantugou ezdou batere louak harzen; khassou éguiten dou bethi. Beldourrak houra ikhouz aguertzen guelditzen ditou ouhouynak. Hounada, sinhetz zazie baynan salbayia da. Laboraribatek anhitz gauza zakienak zioen oussou bere semer Tantugou segour lourreko frouten beyrazaleek hobenena zela.

Ouztarilako egouerdi ondo batez, ardi tropa bat alhazen mendi baten gagnian; arzaina louak hartou zien; iratzarziarekin, ikhousi zien Tantugouk ihez eguiten ziela oyhanerat bourouz. Izan zen igoual arra ezagoutourik arzaynez mendi artebatetako planada pollit batean, etzanik arroka baten gaynean.

Orayno egoun, Pyrénéko herri zonbeytetan, haurrak beldour dira harentzat. Noiz ere mouthiko ttipi batek edo neskato ttipi batek ezpeitoute obéditou nahi bere amari, errayten diote; Gouardi emak hire bouriari! Oyhou eguiten dioat Tantugouri eta, isstantian, ginen da oyhanetik!

Instituteur: Arostéguy, D que, âgé de 46 ans, né à Domezain. 
Baçuçun lehenago Pyrénées erri batian guiçon bat eta émasté bat biciki çaharrak ciénak. Etcicien etché gnimigno bat, baatcé bat eta behi bat bécik. Béna çombat nahi praoubiak içan citen zokorritcen citcien bethi hek béno praouiagouak ciénak. Egun batez, lurra koukoutia çuçun élhurrez eta carrougna hari çuçun harria erdiragarri. Herriko yendé guçiak bérotcen hari citçun, yatiaékin eta édatiaékin; abératz citçun eta iruz. Ulhunduriaékin, bi piayanthe urhun danik yiten ciénak, nahi ukhan cicien lekhu hartan guelditu, hotz baitçuten eta gossé; yo cicien borta batian, guéro bezté batian eta oono beztétan: nihoukéek etcitcien nahi utci zartceat, ez déus éré eman yatéat. Kassatiak orotaï, perzéguitiak chakhurrez, bi eztranyerrek ez çakicien noat youan, noïz eta ere kaoussitu baïtcien erriaen baztérrian praoubé cien guiçon haen eta émasté haen etchiaen aïtcinian. Entçutiaékin héen plégniak, senharra leyhouan yarri çuçun heer oyhu itéko, eta haen émastia youan çuçun fite bortaen idékitéat. Galdein gabé hek nor cien, sarhaaci citcien eta yarhaaci beren zu pasterrian; eta guéro cerbitehatu ceïcien eznia eta çounbeït gaztaina, çusténak oro. Béna ordian, gure Yaüna, hua çuçun Youandéné Pétiriékin, yékiten tçu, buria, oro argui arguia, eta erraiten deiçu: ciek, praoubiak ciesté eta hounak; cien aissouak abératzak tçu eta gachtouak.

Yaincouaren yusticia hin daila.

Béhala lurra ikharatu çuçun, yéssus eta youandéné Pétiri ézeztatu citçun eta bi çaharrak bélhaouriko erori citçun. Bihamun goïcian, nahi ukhan çuténian youan érrirat, yakitéko cer passatu cen, ez cicien ikhussi etcheïk bathéé: héén plaçan, çuçun hur gueldi bat, égun éré atzemaiten déna Barbazan erriaïn eta St Bertrand-de-Comminges hiriaïn artian.

Behartci lagundu maléoussak.

\section{Tantugou Altamann}

Altamann çahar bat tçu gordatcen dena oyhanetan. Beyratcen tci elguiak eta pentciak ez deçaten han déüz ébatz. Oguiak ébakiak diéniann édo belharra ébakia, naoussiak oro bildu artio, Altamann étçu batéé lokhartcen; bethi gouaïtiann daouçu. Altamann aguerth dainn beldurrez egoïten tçu ouhouignac. Altamann hounaou çu, sinhexaçu, baina bassaou çu. Laborari baték biciki gaouça çakiénak erraïten cicinn ardura bere semer Altamann cela séhur lurréko fruitien béiraçaléik hobéna. Uztaillako ilhabéthienn egouerdi ondoatez, ardi tropaat halhan çun mendi baten gainian; artçaigna lokhartu çuçun: iatçartçiaékin, ohartu çuçun Altamann ihessi youiten cela oyénéko aldéat. Berdin éçaoutia içan çuçun artçaignez sohapilla poullit batian, étçana arroka baten gainian. Oraiéé ohono, Pyrénéko çumbeit erritan, haourrak haïndako beldur tçu. Mutiko ttipi batek edo neskatoché ttipi batek ez tiénian nahi sinexi amari, amak erraiten dakoçu: go émak hiri! Oyhu iten dakoat Altamanni, éta behala yien duk oyhénétik.

Instituteur: B. Tambourin, 49 ans, né à Hendaye Lucine, Haranburu, Plachot 


\section{Barbazaneco Guerthacaria}

Baciren berce ordus Pyrénéetaco herri batean guiçon bat eta emazte bat biciki çaharrac cirenac. Ez çuten ontansuntçat etche ttipi bat, baratce bat eta behi bat baicen. Bainan, cembat nahi pobre ciren, sokhoritzen ciusten bethi berac baino pobreago cirenac. Egun batez, elhurra cen, harrien arrailtceco horma ari cen. Herrico yende guciac suphasterrean zauden berotcen, ongui yaten eta edaten çutelaric, aberatx eta uros ciren. Ilhun cerratcean, bi bideyant urrundic heldu cirenac nahi izan ciren guelditu lekhu hartan; ceren goseac eta hostuac baitciren; yo çuten bortha batean, berriz bertce batean eta oraino bertce batçuetan ere ba: nehorc ez cituen nahi izan barnerat sartcerat utci, ez eta deusic yaterat eman. Alde gucietaric khasatuac, chakur hassarre batçuez seguituac, bi bidayant arrotzec ezçakiten norat yoan, noizeta ere khausitu baitciren herriaren basterrean, guiçon eta emazte pobre hekien etche aintcinean. Eztranyer hekien plaïnuac entçunic, senharra athera cen leihora, hekiei erraitera guelditceco, eta aldiz emaztia yoan cen fitez bortharen çabaltcera. Batere galdeguin gabe nor ciren, sar eraci ciusten eta yar eraci suphasterrean; guero eman çacoten yaterat esne eta gastena, heiec çutenetaric.

Bainan orduan, Jesu-Christo gure Yauna, ecen hura cen, Yondoni Petrirekin, yaiki cen, buruac distiratcen çacolaric; eta erran çacoten guiçon eta emaste hekiei: "Çuec, pobreac eta onac çarete; çuen aüsuac aberatxac dire eta gaichtoac. Yaincoaren yusticia içan dadiela eguina!"

Hainbertcerenarekin lurra ikharatu cen, Yesus eta Yondoni Petri galdu ciren handic eta bi guiçon eta emazte çaharrac belhaunico erori ciren..... Biharamun goizean, nahi içan çutelaric yoan herrira, yakitera cer phassatu citekeen, ez çuten ikhusi edo aurkhitu etcheric batere: etchen lekhuan hur bat haundia bacen, çoina oraino egungo egunean ere khautsicen baita Barbazaneco herriaren eta San-Bertrand-de-Commingeco hiriaren artean.

Behar dire sokhorritu malerus direnac.

oOo

Tantugou da guiçon çahar bat oihanetan gordetcen dena. Alhorrac eta phintceac beguiratcen ditu amoreagatic eta handic nehorc deusic ez deçan ebatx. Noiz eta ere baitire oguiac phicatuac edo belharrac ebakiac, hec guciac nausiac bildu artio, Tantugou ez da batere lokhartcen; bethi guardian dago. Harren ikhusteco beldurrac urruntcen ditu handic ohoinac. Ona da Tantugou, sinhetxaçue, bainan salbaya da. Asco gauça çakien laboraribatec erraiten çacon ardura bere semeari, Tantugou cela lurreco fruituen beguiraleric hoberenena. Ustarilaco hilabeteco axalde batez, ardi tropabat alha çagoen mendi batean, artçaina lokhartu cen: iratçarciarekin ikhusi çuen Tantugou oihanerat escapatcen cela. Berdin eçagutua içan cen balea pollit batetaco artçainez, etçana çagolaric harroca baten gainean. Egungo egunean oraino, Pyrénéetaco çombeit herrietan, haurrac beldur dire harentçat. Noiz eta ere mothico edo nescato ttipi batec ez baitu nahi obéditu bere amari, amac erraiten daco: Guardia hiri! Tantugou ekhararasten baituk, eta, istantean, yinen baita.

Instituteur: Puyade, Michel, né à Gotein-Libarrenx (Basses-Pyrénées) le 14 octobre 1840 


\section{Barbazaneco ichtorioa}

Bassèn léhènn, Pyrénétaco herri batiann, guissounn bat eta émasté, çoïnac arrass çahartiac béïtsirènn. Etçutènn étché tchipi bat béçic, baratcé bat éta béhi bat béçic. Béna çombat praoubiac içanic ere, émaïtènn çoutènn béthi amoïna bérac praoubiagouér. Egoun batéz, lourra élhourréz coucoutia çènn éta kharrougna ari cènn harriac érdiratcéco phoundouradio. Hérrico yèndé gouçiac bérotçènn ari cirènn, yatènn éta édatènn, ari çirélaïc. Abératss éta irouss çirènn. Ilhounnçiann, bi bidayènt, ourhounic heldou cirénac, nahi oukhançoutènn guélditou lekhou hartann, çèndaco éta gossé éta hotz baïtcirènn; bortha bat yo çoutènn éta guéro béssté bat, éta guéro béssté batçou: néhoc éré étçoutènn ssartcéra outsi, éta és déouséré yatéra émann. Orotaïc kassatiac, chakour gaïtz batçous séguitiac, bi ésstranyérrac étçakicièn nourat youann noïz et éré guérthatou baïtçiren herriarènn haïndico aldiann, guiçonn éta émasté haïn étchiaïn aïtssiniann, çoïnac praoubiac baitsirènn. Héènn pléygniac intçoutiann, guiçona léyhora yalgui çouçoun héèr oyhou iteko, éta haènn émastia yoann çouçoun fité borthaïn idéquitéa. Héèr galdein gabé nor çirènn, béré étchènn ssar araçi éta yar araçi çoustènn, guéo émann çéétenn éssnia éta gastagna çombaït, çousténac oro. Béna ordiann Gouré Yinco Yaüna (- çoïna béitcènn yoandané Pétri équinn)- choutitou cènn, bouria anhitz brillantic, éta érrann céétènn: "Çièk, praoubiac sisté éta hounak; - sièn aïçoac abératssak çou éta gaïstouak, Yincoaren youssticia ina içanbedi!" Mémènnto bériann, lourra ikharatou cènn. Yésous-Yaouna éta Yoandané Petri galdouçirènn, éta bi çaharrak bélhaounico yarri cirènn. Bihéramounn goïçiann, herrirat yoan nahi oukhann çouteniann yaquiteco çè igarançenn, etçoutènn etchéric batéré ikhoussi: étchènn lekhiann baçènn léissé handi bat, çoïna guérthatsèn béita orono égoungo égounian, Barbazanéco herriaïn éta Ssan-Bértrann Commingéco hiriarènn artiann. Praoubiac béhardira ssokhorritou.

\section{Tantougouènn ichtorioa}

Tantougou guiçonn çahar bat da, oïhanétann gordatsènn déna. Béiratsènn tou alhorrac éta péntsiac, déousérè ébatss ésdéçatènn. Oguiac ébaquiac édo bélharra pphiquatiac, oro barnéat ssarthou artio naoussiac, Tantougou béthi iatçarria do; béillatçènn dou béthi. Houra ikhous béldourra ouhougnac béguiratçènn tou. Ssinhestènn ahal douçié houna déla, béna ssalbaya douçou. Anhits gaouça çakiènn laborari batèc, érraiten çééçoun ardura béré sémer, Tantougou sségourki lourréco frouitiènn béguiraléic hobénéna çéla. Ousstaillaco égouérdi ondo batés, ardi tropa bat alha çènn oyhann cassko batiann; arçagnac loc hartou çouçoun; iratçarsiann, ikhoussi çiçinn Tantougou oyhanbatétara yoaitenn. Eçagoutia içann cènne bésste artçagnès éré, plano édér batiann, harroca batenn gagniann étçanic. Orono égoungo égouniann éré Pyrénétaco herri çoïnbat étann, haourrac haïnn beldour dira. Noïz éta éré mouthico édo néchkato thipia èsdouteniann nahi obéditou bérenn amari, amac érraïten dio: Gogoemac hiri! oyhou éguinènn dakoat Tantougouri, éta mémènto bériann yinènn douc oyhanétic! 
Baçuçun bestorduz Pyreneac deitcen dien Borthu ondouetaco herri batian, guiçoun bat eta emazteat biciqui çaharrac. Etcicien etche chipignoat, baatceat eta behignoat beicic. Bena cembat nahi praoube içanaatic, halere laüntcen citcien bethi berac beno praoubiagoac. Egun batez, lurra coucoutia çuçun elhurrez eta harroquen erdiatceco kharrouign bat aï çuçun. Herrico yende guciac aï citçun yaten eta edaten beotciaequin; abeaxac eta iroussac citçun. Ilhuntcian, bi bidayant urhundic heldu cienac, nahi içan citçun baatu lekhu hartan, ceren hotz eta gossé beitcien; yo cicien borthaat, guero besteat eta fountxian beste hanitz oano; nihourc etcitcin sartceat utci nahi oukhan ez yateat eman. Ootaïc khassatuic, hersatuic chakhur malesiatiez, bi estranyerrec etzaquicien nouat youann, nouiz eta ere errecountratu beitcien herri basterrian, errandelaco guiçoun eta emazte praoube heyen etchiaen aitcinian. Heyen plaigniac entçunez, senharra yelkhi çuçun leihora heyer oïhu iteco eta emaztia youan behala borthaen çaaltcera. Galde in gabe nour cien sarraazten tcie eta yar aasten suphasterrian; guero cherbutchatu deicie esnia eta gaztagna çoumbait, çutena oro. Bena ordian, Yesu-Christo goure Yauna, - Yondoni Phetri eta houra çitçun- yeikitcen tçu, buria argui arguia eta erraiten deïçu: "Ciec praoubiac eta hounac ciezte; cien aiçouac abeaxac eta gaichtouac tçu. Yncouaen yusticia içan daillala inic!" Hambestenaekila, lurra ikhaatu çuçun, Yesus eta Yondoni Phetri galdu citçun eta bi çaharrac belhaïco erori... Bihamen goïcian, youan nahi içan cienian herrirat yaquiteco cer igaan cen, etcicien guehiago etcheïc ikhoussi: heyen orde baçuçun our barna eta gueldi bat çouign ikhousten beita egun oano Barbazaneco herriaen eta Sen Bertran Commingeco hiriaren artian.

Behartcie sokhorritu maleoussec.

oOo

Tantugou çu guiçoun bat oyhenetan gordatcen dena. Elguiac eta phinciac beyatcen tci, yeus ebax ezteçaten. Oguiac ebaquiac dienian edo belharra phicatia, naussiaz oro bildu artino, Tantugouc etci loïc batere iten; bethi goueytian daoçu. Haren ikhousteco beldurrac urhuntcen tci ouhougnac. Hounaouçou, seour içan cite, bena baï salbaya ere. Biciqui gaouça çakien laborari batec, ussu erraiten cien bere semer Tantugou cela seurki lurreco frutien beyaçaleic hobeena.

Uztaïlaco atsalde batez, ardi tropaat alha çuçun mindi batian; arçagna lokhartu çuçun. Iatçarciaekin, ikhousten tci Tantugou oyhenetalat ihes iten. Beste arçaign batçuc ere eçaoutu cicien plano poillit batetan etçanic arroka baten gagnian.

Egungo egunian oano, bortu ondoco herri batçutan, haourrac beldur tçu haïndaco. Mouthico tchipi edo nescatoche tchipi batec espadou ama sinhexi nahi, amac erraiten dacoçu: Gooemac (edo gooemaneçan), oïhu inen diat (edo inen dinat) Tantugoui eta behala ynen duc (edo dun) oyhenetaïc.

Pitrau, 21 ans, né à Sainte-Engrâce (B.P). Coll.: Goyheneix Jean-Pierre, ex instituteur, maire de la commune, né à Arbouet, commune voisine 
Population: 805

Bazitzün lehen müntañetako herri batian gizün bat eta emazte bat biziki zaharrak zienak. Etzizien etche chipiño bat, baatze bat eta behi bat bezik. Bainan, zunbat nahi praübiak izan zizten, sokhorritzen zitzien bethi hek beno praübiaüak zienak. Egün batez lürra elhürrez kükütüik zuzun eta kharruña ari zuzun harrien erdiraazteko phündüradino. Herriko jendiak oro berotzen zien, jaten eta edaten züten; aberats eta irüs zien. Ilhüntzian, bi bidayant ürhünetik heldu zienak nahi izan zizien baatu lekhu hartan, hotz eta gose zielaküan; jo zizien bortha batian, geo beste batian eta beste batzütan oono: nihük etzüzten nahi ükhan utzi sartzea, ez eta ere etzakozien deüsik eman ni ükhan jatea. Igorriak orotaik, segitiak zakhür oldartiez, bi arrotzek etzakiten noat juan; heldu zienian herriaren burura, gizun eta emazte praübe heen etchiaen aintzinea. Entzutiaikilan hein pleintak, senharra jarria zuzun leihuan eta haen emaztia juana zuzun fite borthaen idekitea. Galdein gabe nor zien, sarraazi zitzien eta jarraazi supasterrian; geo eman zeizien jatea esne eta zünbeit gastaña züztenak oro. Ordian, JesüKrichto güre Jauna - hüa züzün Jüandeni Petiriikin - jeiki züzün büria dichtirant dichtiranta eta erran zeizün: "Ziek zizte praübe eta hün; zien aizüak aldiz abeats eta gaichto. Jinküaren justizia jin dadila“. Haimbestenaïkin lürra ikhaatü züzün, Jesus eta Juandeni Petiri itzaldu zitzun eta bi zaharrak erori zitzun belhaünko... Bihaamün goizian, juan nahi ukhan zutenian herrirat ze igaan zen jakiteko, etzizien ikhusi etcherik bihibat: heen lekhian, bazuzun lako bat, oono oai kausitzen dena frantsesez Barbazan deitzen den herriaren eta Saint-Bertrand-deComminges deitzen den hiriaren artian.

Behar tüzi laündu beharrak.

$\begin{array}{ll}\text { Alphabet employé: } \\ \text { Lettres } & \text { Valeur en français } \\ a & a \\ b & b e ́ \\ z & c e ́ \\ d & \text { dé } \\ e & e ́ \\ f & f e ́ \\ g & g u e ́ \text { (même devant } i \text { et é) } \\ h & \text { hé (aspiré) } \\ c h & c h e ́ \\ i & i \\ j & y \text { ordinaire ou } j \text { enfantin } \\ k & k a\end{array}$




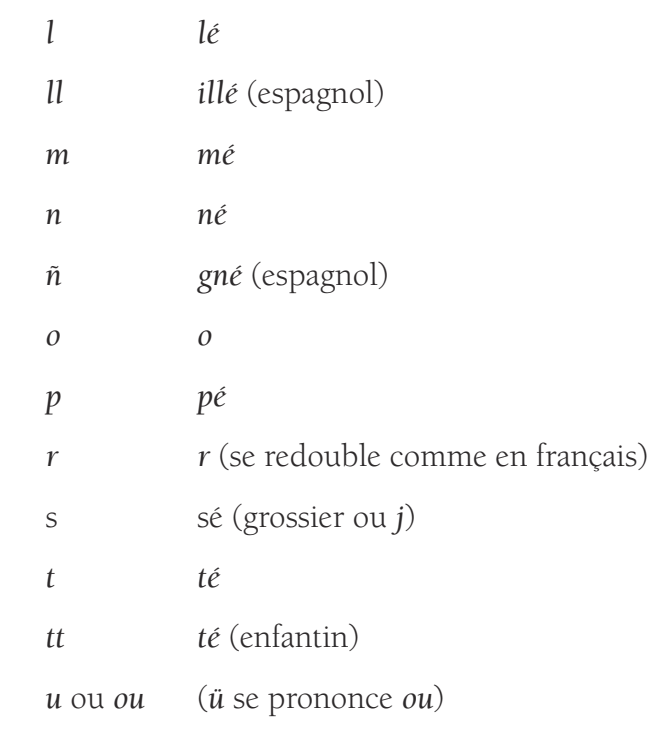

Chaque lettre conserve toujours le son de l'alphabet.

oOo

Tantugü düzü gizün zahar bat oihenetan gordatzen dena. Beiratzen tzi elgiak eta pheintziak ühüñek eztezaten deüs ebats. Ogiak ebakiak dienian edo belharra, oro naüsiaz bildiak izan artio, Tantugük etzi loik iten; beilan dauzü bethi. Hüa agertzen ikhüsteko beldurrak baraazten tzi ühünak. Hüna duzu seür, bainan basa. Biziki gaüza zakien laüari batek erraiten zizin üsü bere semer Tantugü zela seurraz lürreko fruitien beirazaleik hobeena.

Üztallako atsalde batez, ardi arthalde bat alha zagon müntaña baten gañan; artzaña lokhartü züzün: iatzartziaikin ikhusi zizin Tantugü espakatzen oiheneat. Hala hala ezaütuik izan zuzun ülhañez ordoki pullit batian etzana harroka baten gañan. Eün oono, müntañetako herri zünbaitetan, haurrak haren beldur tzu. Müthiko edo neskatoche ttipiñoatek eztienian ama sinhetsi nahi, amak erraiten dakozu: "Oihü iten dakoat Tantugüri eta behala jien duk oihenetik.

Instituteur: G. Istebot, 25 ans, né à Orègue (Basses-Pyrénées) 
Baçuçun beste orduz, Bortuco herri batetan guiçon bat eta emazte bat hanich çaharrac. Etcitcien etche chipi bat, baratce bat eta behi bat baïcic. Bena çoumbat nahi praübé cien, sokhoritcen citcien bethi berac beno praübiago cirenac. Egun batez lurra elhurrez gorderic çuçun eta kharrougna ari çuçun harrien erdiratceco guisan. Herrico gentiac oro berotcen ari çutuçun, jaten eta edaten, aberats eta irouz çutuçun. Ulhuntcian, bi voyajur huruntic giten cirenac nahi içan çutuçun baratu lekhu hartan, ceren gosse eta egarri betciren. Jo cicien bortha batetan, guero bezte çoumbaytetan: ihourquéréc eztcitcien nahi ukhen utci sartcera, ez deüzéré eman jatera. Orotariq acassaturic chakhur gaïchto eli bat ondotiq, bi estrantgerec eztçaquicien nourat joun, nouïs eta ere heltu betciren herriaren basterrila, guiçon eta emaste praübe ciren hayen etche aitcinera. Entçunic hayen plagnic, senharra joun çuçun leyhora deitceco eta emastia joun çuçun bortharen salhu çabaltcera. Batere galdeguin gabe nour ciren, sar araci citcien eta jar eraci suthoundoun; guero cerbutchatu çacocien ezne eta gasteigna çoumbaït, hec çusten gaiçac oro. Bena ordin, Jinco jouna - ceren houra beitcen Joundane Phetiriquin jeiqui çuçun buria argui arguia eta erran çakocien: "ciec praübe ciasté eta houn; cien aïçouac aberats dira eta gaïchto: Jincouaren justicia eguin dadila. Haïn sarri lurra ikharratu çuçun, Jesus eta Joundane Phetiri galdu çutuçun eta bi çaharrac eori belharico.... Biharamen goïcian jouan cienian herrila jaquiteco cer igaran cen, eztcicien ikhousi etcheric batere: hen plaçan baçuçun lac bat, orano egun ere edireiten dena Barbazaneco hériaren eta Saint-Bertrand de Comminges hiriaren artian. Behar dicie lagundu malerousec.

oOo

Tantugou guiçon çahar bat duçu çouin gordatcen beita oyhenetan. Beguiratcen titçu elguiac eta phintciac ezteçaten han deuzere ebats. Oguiac mousturic dienian edo belhara ebaquiric, naussiac oro sar artino, Tantugou eztuçu batere lotcen, bethi iratçarriric duçu. Haren ikhousteco lotsac baratcen titçu ouhougnac. Houn duçu sinhets açu, bena salbage. Laborari batec çouignec betçaquin hanich gaiça cioçun ussu bere semer Tantugou cela segur lurreco fruten beguirariaric hobena. Ustarilaco aratz alde batez ardi tropa bat alha çuçun mendi batetan; artçaigna lothu çuçun: iratçartciarequin ikhoussi çiçun Tantugou ezcapatcen oyheneco alderat.

Ber guizan eçaguturic içan çuçun artçaignetcaz urruphea pouillit batetan arroca baten gagnen etçanic. Orano egun bortuco herri çoumbaytetan haurrac haren lotxa tutçu. Nouiz eta éré mouthico chipi batec edo nescatoche batec eztianian nahi ama obeditu, hounec errayten dacoçu: "Abisadi jin erasten diat Tantugou eta berhala ginen duc oyhenetic.

Nota - En Basque, il n'y a pas de e muet- Par conséquent, les accents sur les e sont inutiles.

Instituteur: Hastaran, 35 ans, né à Viodos-Abense Coll.: Paguéguy Arnaud 
Bacitçun beste orduz Pyrenesseco bortietaco herri batian guiçun bat eta emaste bat arras çaharrac cienac. Eztcicien etche gnimigno bat becic, baatce bat eta behi bat. Bena çumbat nahi praube içanatic, laguntcen citcien bethi berac beno praübio cienac. Egun batez, lurra cucutia çuçun elhurrez, eta kharrugna iten cin harrien erdiratceco hegnan. Herrico yendiac oro beotcen citçun yatiaïkin eta edatiaikin, abaats eta irus çitçun. Ilhundirian, bi piayant heldu cienac urhunt danic nahi ukhan cicien baatu toki hortan, ceren eta hotz eta gosse bitciren, yo cicien borthaatian, gueo besteatian eta besteatcietan oono: nihoukec ez tcitcien utci nahi ukhan sartceat, ez eta ere heer deüs eman yateat. Orotaïc kassatiac, chakhur erreboltatiez seguitiac, bi estranyerrec ez tçakicien noat yuan, noiz eta ere caüsitu bitciren herriaïn bazterrian, guiçun eta emazte praübe ciren heyen etchïan aïtcinian. Entçutian heyen plagniac, senharra yuan tçuçun leihora heer oïhu iteco, eta hain emaztia yuan tçuçun fite borthaïn ideïtcera. Batere heen galdeïn gabe nurtcien, hec saraci citcien eta yarraci beren supazterrian; gueo heer cherbitchatu esnia eta çumbeït gaztagna, oro cuten gucia. Bena ordian, Gure-Yaüna -hua cuçun Yondoni Petrikin- chutitu çuçun, buria brillanki, eta erran ceïçun: "Ciec praübiac eta hunac cizte. Cien aïçouac abaatsac eta gachtouac tutçu iYinkuain yusticia in daillala. Berehala lurra ikharatu çuçun, Yessus eta Yondoni Petri eceztatu citçun eta bi çaharrac behaünca erori citçun...

Biharamun goïcian, noiz eta ere yuan nahi içan bitçuten herrirat, yakiteco cer passatu cen, ez tcicien ikhusi etcheïc batere: heen orde, baçuçun ur gueldi bat oono egun caüssitcen denhua Barbazanéco herriaïn eta Yondoni Beñat Commigneco hiriaïn artian. Behaïcie malhurussec lagundu.

\section{oOo}

Tantugou guiçun çahar bat duçu gordetcen dena oïhenetan. Elguiac eta phintciac beatcentci aments ez deçaten deüsse ebats. Noiz eta ere oguiac phikatiac bitira edo belharra ebakia, naüssiac ukhan artio oro khendiac, Tantugu ez duçu batere lokhartzen. Iatçarria doçu bethi. Haïn ikhusteco beldurrac guielatcen tci ouhougnac. Hua hunaüçu, sinhets çacie hori, bena salbayaüçu. Biciki gauças çakien laborari batec erten cin ardura bere semer Tantugou cela seürki lurreco fruïtuen beïratçaleïc hobeena.

Ustaillaco hilaïteco atsalde batez, ardi tropa bat alhatcen çuçun mindi baten gagnan; artçagna lokhartu çuçun: iatçartciaikin, ikhusi cin Tantugu ihes baçuala oïhanari buruz. Berdin eçaütia içan çuçun artçagnez ibarre poullit batian etçanic harrokaten gagnan.

Oono egun, Pyrenesseco bortietaco herri çumbeïtetan, haürrec haïn beldurraicie. Noiz eta ere mutico gnimigno batec edo nechkatoche gnimigno batec ez dinian obeitu nahi bere amari, hari erten dacoçu amac: Khassu eïn tçac (ou bien) khassu eïn tçan hiri! Oïhu iten dacoat -ou bien daconat- Tantuguri, eta, behala yinen duc-ou bien dun- oïhanetic.

Instituteur: Etcheberry (Justin), âgé de 21 ans, à Macaye Coll.: Sallaberry Pierre, 49 ans, laboureur 
Lehenago Pyrénéetako herri batetan bazuzun zenhar eta emazte batzu biziki zaharrak zienak. Ontazunik etzizien etche ttipi bat baratzeatekin eta behi bat bezik. Bena zoumbat nahi praube izanike, berak beno beharragoak laguntzen zitzien.

Egun batez lurrac elhurrez koukoutia zuzun eta iten zin kharrouïnbat, harriak erdiatzeko phundaraïnokoa. Herriko yende guziak berotzen zizun, onza yaten eta édéaten zutelaïk, zen eta abeatz eta irouz bitzitien. Ilhunian bi bogajür urhunetik heldu zienak herri hartan arreztatu zitzun goze eta hotz bitzuten: borthat yo bezteat yo herri guzia courritu zizien, bena nihourek itzitzien nahi oukhan errezeïtu, ez etare yateat eman. Orotaïk khazatuïk et chakhurrez houlatuik, bi bogajur hek etzakizien noat yoan, arribatu zitienian herriaïn phuntalat delako zenhar eta emazte praübe heïn étche aïtzineat. Zenharrak hein pleïnta intzunik leïhiria zaldiezin eta emaztiari bortha zalazi. Guero galdin gabe nor zitien ez nountik heldu zitien, beren etcheat zarrazi zitzien eta yarrazi oundouan, eznez eta gaztagnaz ountza azeazi eta hitz batez erraiteko juten gaüza orotaïk eman zeizien.

Ordian Jesu-Christo (zeneta houa bitzen yoandene Petirikin) chutitu zun eta buria ingurukatia zielaïk arguizko khoro batez erran zeizun: ziek praübe eta bihotz houn zizte eta zien aïzouak abeatz eta gaïchto. Yinko hounaïn yustizea kompli bedi! Eta hitz horiek erran bezaïn lazter lurra ikharatu zuzun, Jesus eta yoandene Petiri altchatu zitzun zerurat eta emazte hek belhaunikatu. Bihamounian herrirat nahi oukhan zutenian youan yakiteko zer phazatzentzen etzizien etcheik ikhouzi bena baï our handi batek itho zitiela oro.

Oraï ere ono our houa agueri uzu Barbazan eta San Bertrand-de-Comminges deitzen dien bi hirien artian. Hounek eakouzten dakuzu behar tula praübiak lagundu.

\section{oOo}

Tantougou zen guizon zahar bat oïhanetan bizi dena. Pentziak eta elguiak beratzen tzi ezdezaten deuzik ebatz. Etzi loïk iten belharrak eta oguiak ebakiak dienian, hek naüziak barneat ezar artino, bethi dembora hetan gaüak beilatzenzi. Ouhougnak haïn ikhouzteko beldurrak atzikitzentzi. Penzatzen ahal zu houna dela bena hala baza. Laborari yakintzu batek eta ezkolatu batek, erraïten zeïzun uzu bere zemer Tantougou zela, lurreko errekolten beïrazale hobena.

Uztaïlako atzaldeatez, ardi tropat montagnatetan alha zuzun, artzagna lozelaïk jatzartu zenian ikhouzi zizin artzain hak Tantugou oïheneat ezkapatzen eta bezte hanizko artzagneke ikhouzizic eta aïze ezaoutu arrokatzutan etzanik.

Ono egungo egunetan Pyrenetako hanizko herrietan haourrak haïndako beldurzu eta badenian haour gnimigno zoumbeit ama zinhetzi nahi ezdiena, ama hak erraiten dakozu: gomak hiri: Tantougouri oïhu iten diat eta behala hemen izaïn douk. 


\section{Barbassaneko ichtoria}

Bacen beste orduz Pyrénéétako herri batian guiçon bat eta emastebat biciki çaharrak cienak. Etçuten etche gnimignobat becik, batce bat eta behi bat. Bena, hain praoubiak içanaatik, sokhorritcen çuzten bethi hek beno praoubiagouak cienak. Egun batez lurra koukoutia cen elhurrez eta kharrougna ari cen harriak erdiastekan. Herriko yende guciak berotcen ari cien, jatiakin eta edatiakin; abeatsak cien eta irous. Astirian, bi bidayant jiten cienak urhunetik nahi oukhan çuten baatu lekhuhartan, céen éta hotz bitcien eta gossé. Yoçuten bortha 'tian, guio beste 'atçutan ôno: Nihourek etçuzten utci nahi oukhan sartcea, ez yose eman jatea. Khassatiak orotaik, séitiak chakhur errabiatiez, bi estranyerrek etçakiten noat youan, guerthatu cienian herrian heguian, praoubiak cien guiçoun haine ta emaste hain etchian aintcinian. Untçutiakin heien oihu minak, senharra jarricen leihouan, héier oihu iteko, eta hain emastia youan fité borthain çaalcea. Héier galdin gabe nour cienn, sarraci çusten eta yarraci suphasterrian; guio, cherbutchatu ceiten esne eta gaztagna: çuztenak oro. Bena ordian, Gure Yauna, -houa cen, youan dene Phetirikin- jekicen, buria argui arguia, eta erran céen: "Ciek, praoubiak cizte eta hounak; cien aiçouak abeatsak dira eta gaichtouak Yinkouain Justicia eguin dailala!" Behala lurra ikhaatu cen, Yesus eta Phetiri galdu cien eta çaharrak erori cien belhauco... Bihamoun goician, nahi oukhan çutenian jouan herrirat jakiteko ce igan cen, etçuten ikhoussi etcheik baté: heien lekhian, bacen our bat, ôno egoun hatcemaiten dena Barbassaneko herrian eta San Betran Comingeko hiriain artian. Maleoussak behar dira lagundu.

\section{Tantugouin ichtoria}

Tantugou da guiçon çahar bat gordatcen dena oihenetan. Beatcentu elguiak eta phintciak ezteçaten han jose ébats. Oguiak moustiak dienian edo belharra epheina, naoussiak oro idokiak oukhan artio, Tantugouk eztu loik bate iten; beillan daou bethi. Hain aguertcen ikhousteko beldurrak atchikiten tu ouhougnak. Houna da, sinhetsacié bena bassa da. Anhichko gaouça çakien laouri batek erraiten cien ussu bere semer Tantugou cela seurki lurreko fruten beaçaleik hobena.

Ustaillako egordi oundo batez, ardi tropat alhancen, mindi baten gagnian; artçagna lokhartu cen: iatçarciakin, ikhoussi cien Tantugou ihes iten ciela oiheneat buz. Eçaoutia içan cen hala hala artçagnez errepea pouillit batian, etçana arroka 'ten gagnian.

Ono egun, Pyréneetako çombeit herrietan, haourrek hain beldur dute. Mouthiko gnimignobatek edo neskateuche gnimignobatek ez tienian sinhetsi nahi bere ama, erraiten dako: "Gouardia emak hiri! Oihu iten dakoat Tantugouri eta ichtantian jien douk oihenetik!

Instituteur: Guichandut, âgé de 27 ans, né à Ilharre. Coll.: Ahanu, âgée de 80 ans 
Barbézanén istoria (1)

Béstordus baçén Pyrénéco herri batétan giçoun bat éta émastéat biciki saharrac. Etsutén étché chipi bat, béhi bat éta baatcé bat béicic; béna, soumbat nahi praoubé içanic éré, bérac béino praoubiagouac chocorritsén sustén. Egun batès lurra élhurrés coucoutia sén éta harritséco carrougna égitén sien. Hérrico iéndiac oro bérotsén ari sirén, iatén éta édatén sustélaïc: abéatch éta urous sirén. Ilhuncian, bi bidéiant urhunétic helduciénac nahi oukhan sutén gélditu lékhu hartan; hots éta goché sirén. Jo suten bortha bat, besté bat éta oano bestéat: nihouké étsusten nahi utsi chartséa es éta éman iatéa. Orotaic igorric, chacur achaldatiac ondoti, bi arrotséc étsakiten noat jouan, hérrian bastérréat siénian caouchitu siren chenhar émasté héén borthan. Ensunic héén plégniac laster chenharra jouansén leïhora oïhu éitia éta émastec bortha én sabalcéa. Galdéin gabé norc siren, charraraci sustén éta iarraraci su pastarrian; géro, éman sacotén ïatéa gastagna soumbéit éta échné: éspitsutén bestéï. Ordian Jésu-Cristo (bi arrotsac Jésu-Cristo éta Joan-déné Pétri siren) chutitu sén, buria argi argia éta érran sirén: "Siéc praoubé siré, baïnan houn; sién aịçoac abérach diré baïnan gaïchtouac; Jincoén justicia égin bédi”. Haïn béstéékin lurra iccaatu sén éta bi saharrac belhaïco érori sién... Bihaamoun goïcian hérrirat jouan sirénian iakitéco cér agitu cen, etsén étcheïc batéré agéri, héén léchkian bassén laco baat oaï ono éré Barbezanéco herrian éta San-Bertran-de-Comminye én artian déna.

Béhar da laündu béharrétan déna.

(1) La lettre $g$ a toujours le son dur.

\section{Tantugou én istoria (1)}

Tantugou sahar bat da coutsén baïta oïhénétan. Bégiatséntu pinciac éta élgiac amoéagatic éta déoujéré ébach estéçatén. Ogiac éta bélharrac moustu oundouan naoucic oro éréman artio Tantugou estu loïc baté éginén. Harén béldurrac ouhougnéc huillancétic baratséntu. Tantugou houna da, chinésdéçacié béna bacha da. Laborari batèc béiçakien gaouça biciki, erraïten sérén uchu béré chémér: Tantugou lurréco frutien bégiaçalé obéna cela.

Açarouen égouardi ondo batés ardi tropa bat alhacélaric mindi batétan arsagna lothu sén; arsagna iatsari sénian ickouchi sien Tantugou oyhénarat éscapatsen. Tantugou içancen éçagutouric arsagnés naba pouillit batétan harroca batétan gaïnian.

Oaïno aïgun Pyrénéco herri batsuétan haourrac lotcha dira haén; néchcatoché chipi édo mouthico chipi batec estuténian ama chinétchi nahi, amac erraiten diéçu: "Ago! ago! oyhu itén diat Tantugouri instantian jinén duc oyhénétic.

(1) La lettre $g$ a toujours le son dur

Instituteur: Condry, né à Issor, le 29 septembre 1857 Coll.: Inchauspé et Domercq 


\section{Barbassaneco ichtoria}

Baciren lehenago Pyénéétaco herri batean giçon bat eta emaztebat biciki çaharrak cienak. Etçuten etché gnimignobat becik batceat eta behi bat. Bena hain praoubiak içanatik sokhorritcen çusten bethi hek beno praoubiagoak cienak. Egun batez lurra koukoutia cen elhurrez eta karrougna ari cen harriak erdiartekan. Herriko yende guciak beratcen aricien yatiakin eta ediatiakin: abeatsak cien, eta irous. Atsarribuz* bi bidayant yiten cienak urrhun etik nahi oukhan çuten bâtu lekhu hartan cêneta hotz betcien eta gossé. Yo çuten bortha batian gio beste batian eta beste batçutan ôno nihourek etcuzten utci nahi oukhan sartcea ez nahi oukhan yoose eman yatera. kasatiak orotaic seigitiak chakhur errabiatiez bi estranyerrek etçakiten noat youan kaoussitu cienian herrian hégian praoubiak cien giçon hain eta emazté hain etchian aintcinian. Untçutiakin heien plagniak, senharra yarri cen leinouan, heien oïhuteco eta haïn emaztia youantcen fité borthaïn çalcea. Heien galdin gabe nor cien sarraci custen eta yarraci su bazterrian; gio cherbutchatu ceiten esne eta gaztagna, çuztenak oro. Bena ordian Goure Yaouna -houa sen Youan Dene Phetirikin- yekicen buria argi argia eta erran cein: "Ciek, praoubiak cizté eta hounak; cien aiçouak abeatsak dira eta gachtouak. Yinkouain yusticia egin daïlala! Behala lurra ikhaatu cen Yesus eta Phetiri galdu cien eta çaharrak erori cien belhaïco... Bihamoun goïcian nahi oukhan çutenian youan herrirat yakiteko ce igan cen, etçuten ikhoussi echeik bate: hein lekhian bacen phutçu bat ono egun hatcemaïten dena Barbasseneko herrian eta san Bertran Commeinge kohirian artian. Maleoussak behar dira lagundu.

\section{Tantugoin ichtoria}

Tantugou da giçoun çahar bat gordatcen dena oïhenetan. Beatcen tu elgiak eta phintciak han ezteçaten yosé ébats. Ogiak moustiak dienian edo belharra éphéina, naoussiak oroidekiak oukhan artia ez tu loïk bate iten; beillatcen du bethi. Hain agertcen ikhusteko beldurrak atchikitcen tu ouhougnat. Hounada, sinhets acie bena bassa da. Anhitz gaouça cakien laborari batek erraïten cien ussu beré semer Tantugou cela segurki lurreko fruten beaçaleic hobena.

Ustailhako egardi oundo batez ardi tropat alhancen mindi baten gagnian; artçagna lokhartucen; iatçarciakin ikhoussi cien Tantugou ihesiten ciela oiheneat buz. Hala hala eçaoutia içan cen artçain batçuz errépéa pouillit batian arrokaten gagnian etçana.

Ono egun Pyrenéétako combeit herrietan haourrek hain beldur duté. Moutchiko gnimignobatek edo neskhateuché gnimignobatek ez tienen sinhetsi nahi béré ama, erraiten dako: "Gouardiemak hiri! Oîhu iten dakouat Tantugouri eta ichtantian yien douk oïhenetik.

Instituteur: Ducos (Jean), 44 ans, né à Hagetaubin (Basses-Pyrénées) 


\section{Barbanazeco ichtoïa}

Léhen bisi sién Pyrénéco héri batian guizon bat éta émaste bat bisiqui saharac. Etsuten etché chipi bat baatsé bat éta béhi bat baïsic. Béna soumbat nahi praoué isanic é béac béno praouiagouac sécouritsen sustén. Egun batés lura élhurés coucoutia sén éta kharougna ari sen. Hérico yende gusiac yatén éta édaten ari siélaï, béotsen sién; iroussac éta abératsac sién. Ulhuntsé ulhuntsian bi vouaïayur huruntic héldu siénac, nahi isan sién arestatu héri hortan gossé éta otz siélacos; bortha bat étan yo sutén, guéro besté bat étan éta oraïno béste hanichcotan: nihourqué étsutén utsi nahi isan sartséra, es éta déusé yatea éman. Orotaïc khassutuic, chakhurrés yaraïquic, ez yaquin nouat youn, heltsen dia bi estranyérac guizon éta émaste praoué hén etché aïtsinéra soungn héri baster batian béitsen. Hén plagniac entsutiaïquin sénhara léihoula aguertu sén her oïhu itéco, éta emastia youan sén bérhala borthaïn idéquitéa. Galdéguin gabé nour sién, sarassi éta yarassi sustén shukhaldian; guéro éman tsén siénétic, esné éta gastagna soumbéit. Béna ordian Yinco houna -houa sén Simpiéraïquin- yeiqui sén buria oro arguitia éta érantsen: "Siéc praoué éta houn cisté; sién aïssouac abératz éta gaïchto dia. Yincoarén yustissia éguin bédi!” Haïnbértsénaiquin lura ikharatu sén, Yésus éta Simpiéra galdu sién éta bi saharac bélhaïco érori...

Bihamun goïcian, nahisan siénan élissa athila youan, yaquiteco ser passatu sén, étsutén etchéic baté icoussi: hén lékhian bassen léissebat, égun idiéiten déna oano Barbazanéco hériaïn eta Simbertrand-de-Commingéco hériain artian.

Béhar da sécouritu maléousac.

\section{Tantougouén ichtoïa}

Tantugou guizon çaharbat da oihanétan gordatsen déna. Béguiatsentu alhorrac eta phintsiac esdésaten han déusé ébats. Oguiac moustuic diénian édo bélharra ébaquic, naousias oro sarartio, Tantugouc esdu loïc iten; beillatsen da béthi. Ouhougnac esdia aousartatsén, haréc hatséman béldures. Sinhestén ahaltsu guizon houna déla, béna salbayé dusu. Laborari batéc, gaousa bisiqui saquiéna, éraïten sién ussu béré sémer séurqui Tantugou séla luréco frutén béguiaïc hobéna.

Ustaillaco atsaldé batés, ardi saldobat alha sén méndi batian; artsagna lokhartusén: iratsartsiaiquin, icoussi sién Tantugou oïhanéat ihéssi souéla. Esaguturic isantsén béhi sagnés é planada pouillit batian, aroca batén gagnian étsanic.

Oaï é, Pyrénéco héri batsutan haourac lotsa dia haïndaco. Mithico édo néchcatoché chipi batéc ésdiénian ama sinhétsi nahi, éraïten daco: "Khassu isac! Tantugouï oïhu itén dacoat éta béhala, oïhanetic yinén duc! 


\section{Barbazaneco eran sahara}

Bortuko heribatian basien lehenago gizon bat eta emazte bat bissiki sahartiac. Etzuten etche tipibat, baratzebat eta behigno baisik. Bena praoube izanagatik ere laguntzen suzten bethi berak beno praoubiagouac. Egun batez lura thapatia sen elhurez eta éguiten cin kharouinbat harien arrailtatzecoa. Heriko yentiac oro berotzen ari siren, yaten eta edaten sutelarik: aberatz eta uruz siren. Ulhunsian, bi bideyant huruntik hora sienak nahisan siren gelditu toki hartan, ceren hotz eta gose beitzien; yo zuten bortha batian, gero beste batian, eta orano beste batzutan: nehork etziuzten sartzerat utzi, ez eman nahi izan deusik yatera. Orotarik ohiltuac, sakhur gaitz batzus yaraikiak, bi heri kampotar hek etzakiten nourat yoan, noiz eta edireiten beitira gizon eta emazte praoube hen etche aitzinian. Hen oïhuen entzutiaekin, senhara yari sen leihouan deitzeko, eta emaztia yoan sen salhu bortaren sabaltzera. Galdegin gabe nor siren, sar eta yar erasi suzten su ondouan; gero, emaiten dere ezne eta gaztagna senbait, suzten gusiak. Bena ordian, Jesu Krist goure Yauna, -houra sen, Yondane Phetiriekin, - yeikitzen da, buria dena argi argia, eta eraiten dere: "Siek, praoube eta houn siazte; sien ausuak aberatz eta gaisto dira. Yenkoaren nahi eginik izan bedi... Dembora berian lura ikharatzen da, Jesus eta Yondane Phetiri galtzen dira eta bi saharak erortzen dira belhaiko. Bihamen goisian yoan nahisan sienian herila, yakitera ser igaran sen, etzuten ikhoussi etcherik bate: hen lekhian, basen phuzu handi bat, egun orano Barbazaneko herian et Saint-Bertrand-de-Commingeko hiriaren artian edireiten dena.

Behardira lagundu dohatkabiak.

\section{Tantugouen eran sahara}

Tantugou da gizon saharbat nour gordatzen beita oihanetan. Begiratzen tu alhorak eta pentziak amorekatik esdazaten deusik ebatz. Ogiak mousturik edo belharrak ebakiak dienian, hek oro naussiak barnen sar-artino, Tantugou ezda lokartzen batere; bethi bellatzen da. Haren ikhousteko beldurak ohoinac huruntzen tu. Houna da, sinhezcasié, bena bassa da. Laborari batek beitzakien gausa hanitz eraiten sien ussu bere semer Tantugou segurki lureko ountasunen begira salerik hobena sela.

Ustailako arats aldebatez, ardi saldobat mendi batian alhen ari sen; artzagna lokhartzen da: irartzar siaikin ikousten du Tantugou oihanari buruz lasterka ezpakatzen. Issan sen esagutia ilhagnez naba pollit batian, etzanik sagularik botchebaten gagnian.

Egun oraino bortietako heri sembaitetan, haurak beldur dira harentako. Noiz eta mothiko edo nekatoche tipibatek espeitu nahi obeditu bere amari, hounec eraiten deo: Khassu egik! Deitzen diat Tantugou eta, berhala, jinen duk oihanetik.

Instituteur: Armagnague, 26 ans, né à Ainharp Coll.: André Rospide 


\section{Barbazan. eco istorio zaharra}

Bazen lehenago Pyrénées-taco herri batian guizon bat eta emazte bat biziki zahar zirenac. Ez zuten (ou etzuten) etche ttipi bat, baratze bat eta behi bat baïsic. Baïnan, haïn pobre izana.gatic, laguntzen zuten hec baïno pobreago zirenac. Egun batez, lurra elhurrez estalia sen (ou çen), eta kharrugna ari zen harria erdiratzeco maneran. Herrico yende guziac berotzen ziren, yatiarekin eta edatiarekin; aberax eta irus ziren. Ilhuna sartziarekin, bi piayant urhunetic heldu zirenac, nahi ukhhan zuten gelditu lekhu hartan, çeren hots eta gose çiren; yo zuten bortha batian, gero beste batian eta beste batzuetan oraino: Nihoc ez zuen (ou etzuen) nahi

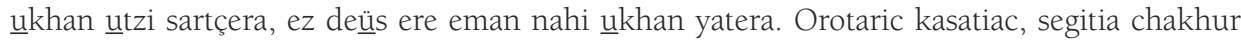
furiüsez, bi campotiarrec etzakiten norat yuan, guerthatu zirenian herri bazterrian, guison eta emazte heyen etchearen aitzinian. Entzutian heyen plegniac, senharra yarri çen leihuan heyen deithatzeco, eta haren emaztia yuan çen laster bortharen zabalzerat. Galdeguin gabe heyer nor ziren, sar-arazi zusten eta yar-arazi beren su bazterrian; guero, çerbitzatu zioten esnia eta gastaïgna zombaït, çuztenac oro. Baïnan ordian, gure Yaïncoa, -hurra cen, Yondoni Petirirekin,- chutitu çen, buria arguiz inguratia, eta erran çioten: "ziec pobre eta hûn çiezte; çien aüssuac aberax eta gaïchtoac dire. Yaïncoaren yustizia eguina izan bedi!” Abilduran* lurrra ikaratzen da, Yesus eta Yondoni Petiri itzali ciren eta bi zaharrac erori çiren belhaünico....... Biharamun goïsian, nahi ukhan çutenian yüan herrirat, yakiteco cer iragan çen, etzuten ikhusi etcheric batere: Heyen lekhian, basen ur handi bat, kaüsitzen dena egungo egunian Barbasan. eco herriaren eta Saint-Bertrand-de-Comminges.eco hiriaren artian. Behar dira sokhorritu (ou lagunduㅡ) malerusac (ou dohacabiac).

Nota. 1. L'orthographe du morceau qui précède est celle qui est généralement adoptée dans le canton de St-Palais.

2. Il faut tenir compte qu'en basque la consonne $z$ a la même prononciation que ç et que ces deux lettres s'emploient indifféremment l'une pour l'autre.

3. La voyelle $\underline{u}$ doit être prononcée ou partout où elle est soulignée.

4. En basque la consonne $g$ n'a jamais le son de $j$.

5. La langue basque ne possède pas d'expressions propres pour désigner les mots français légende et lac.

\section{oOo}

Tantugou da zahar bat gordatzen dena oyhanetan (Prononcez oïhanetan). Beïratzen ditu elguiac eta peintziac ez desaten han deüs ebats. Oguiac phicatiac direnian edo belharra ebakia, naüssiac oro sarthu artino Tantugouc ezdu lo eguiten (ou ezda lokartzen); goardian da bethi. Beldurrac haren ikhusteco aguertzen beïratzen ditu ohoïnac. Huna da, sinhets-assuee, baïnan basa da. Laborari batec anhitz gaüsa zakienac erten çien usu bere semer Tantugou zela 
segurki lürreco fruiten beguirale hoberena.

Ustailleco arratsalde batez, ardi mullcho bat alha çen mendi batian; arzaina lokhartu cen: iratzarciarekin ohartu cen Tantugou ihes zoala oihaneco alderat. Ezagutia izan cen bardin arzaïnez errepira poillit batian, etzana harroca baten gaïnian.

Oraïno egungo egunian, Pyrénées-taco herri batzuetan, haürrac beldur dira hartaz. Noiz ere muthico edo nechcato ttipi batec ez beïtu obeditu nahi bere amari, erten daco: "Goardia emac (ou eman) hiri! Deïthatzen diat Tantugou eta, ichtatian, yinen dục (ou dun) oyhanetic!"

Instituteur: Lartigau (Martin), âgé de 34 ans, né à Luxe Coll.: Héguito (Anne), mère de l'instituteur 
Baçuçun bestordus bassepireneco heribatian guiçonbat eta emastebat biciki çaharac beitcian. Etcicien etche tipibat baicic eta baratce gnobat eta behignobat. Bena combat nahi praube cien sokoritcen cuten bethi hec beno praubiagouak. Egun batez, lura estalia cen elhurez eta karoumbat haritcecoua: herico jente guciac beotcen aicitçun jaten eta edaten aricirelaric; aberats citçun eta irous. Gauan sartciarekin bi boyageurs heldu cirelaric urrundanic nahi uken cicien han baratu certaco gosse eta egarria beitciren. Go cicien bortha batian, guero bestebatian eta bestebatian oraino, nihorkec etcicien nahi uken sartcera utci eta nihoukek jatera eman nahuken.- Kassaturic orotaric, chacurac ondotic igorriric, bi estrangerec hec etçakicien nourat jouan noiz edien beitciren heriaren bastera, guiçon eta emaste prauve hen etche aitcinian. Hen plagniac entçutiarekin, senhara jalki çuçun leihobat etala oihu eguiteco eta emaztia jouan çuçun berhala bortharen idoquitcera bateren galdeguin gabe nour ciren, sareraci çuzten eta jar eraci supasterian: guero cherbutchatu cen esne eta çombait gastagna, çuten guciac. Bainan ordian - Goure Jauna- houra çuçun St Pierraekin - jaiki cen, buria arguis betheric, eta eran ceieçun; ciec, ciste prauve eta prauve hounac, cien aicouac tçu aberas eta gaistouac- Gincouaren justicia içan dadila- berhala lura ikharatcen da. Jesus eta Pierra Saindua jouan ciren airian eta bi caharac jari citçun belharico. Biharamen goician, nahi ukhen cutenian jouan herilat jakiteco cer igaran cen, etçuten ikoussi etcheric batere hen lekhian baçun hour gueldibat oraino egun ikousten dena Barbazaneco heriaren eta St Bertrand Gomminge hiriaren artian. Behar da laguntu malerousac.

\section{oOo}

Tantugou guiçon çaharbat da, coin gordatcen beita oyhanetan. Beguiratcen tu alhorac eta pinciac, amorecatic deusic esteçaten ebax. Nois eta oguiac ebaquiac beitira, edo belhara moustia, guciac barnen eçartio naussiac, Tantugou ezta lokhartcen, atçaria dago bethi. Haren ikousteco beldurac atchikitcen tu ohoinac. Houna da, sinhex ecacie ounxa, bainan salbajia da. Laboraribatec çoinac beitçaquien hanits gauça erraiten cien ussu bere semer: Tantugou cela segurki lurreco frutien beguiraçale hoberena". Ustarilaco arax aldibates, arditropabat alhacen cen bortian, arcagna lo cen, iratçarciarekin ikoussi cien Tantugou eskapatcen oyhanerat. Içan cen guisaberian eçagutia artçagnez ordoki polit batian etçanic harroca baten gainean. Egun oraino Pyrénéco heri coimbat etan, haurrac beldur dira haren. Noïs eta mothiko gnimignobat edo neskatoche gnimigno batec espeitu nahi obeditu bere amari, eraiten da: habisa hiri, deitcen diat Tantugou eta istant berian jinen duc oyhanetic.

Instituteur: Arainty, 45 ans, né à Sauguis (B-P) Coll.: Urruty, aubergiste, 62 ans 
Bacen beste ordous Pyrénéetaco herri batian guiçon eta emazte bat biciki çaharrac. Etçouten etche gnimignobat baïcik, baratzebat eta behi bat. Bena, çombat nahi pobre içan citen, sokhorritcen çousten bethi berak beno pobriagoac. Egoun batez, lourra coucoutia cen elhourrez, eta harrien arrailltzeko kharrougna ari cen. Herrico jende gouciac berotcen çaouden, janez eta edanez; aberats eta urous ciren. Ilhoundirian, bi bidayant ourhounic heldou cirenac guelditou nahi içan ciren lekhou hartan, ceren eta hotz beitciren eta gosse; yo çouten bortha batian, guero beste batian eta beste batçouetan oraino: nehorc ez citien outci nahi oukhen sartcera, ez heyer deus emann jatera. Cassatiac orotaic, perseguitiac chakhour gaïchtouez, bi estranyerec ez çakiten norat jouan, noiz eta ere guerthatou beitciren hirriaren bazterrian guiçon eta emazte pobre etchiaren aitcinian. Entçoutiarekin heyen plagniac, senharra eman cen leyhouan heyen deythatceko, eta haren emaztia joan cen fite bortharen çabaltcera. Heyer galdeguin gabe nor ciren, sar araci çouzten eta jar araci bere sou pazterrian; guero cerbitçatu çakoten esne eta gastagna çombaï, berec çouten goucia. Bena ordian, Jessou-Christo-Goure-Jaouna ecen houra beitcen, Jaoun-Doni-Petrirekin -choutitou cen, bourria cielaric arras distiranta, eta erran cakoten: "Ciec pobriac ciezte eta hounac; cien aiç̧ouac aberatxac ditçou eta gaïchtouac. Jincouaren yusticia eguin dadila!" Haïn laster lourra ikharatou cen, Jessous eta Jaoun-Doni-Petri bichtatic galdou ciren eta bi çaharrac eman ciren belhaounica... Biharamoun goïcian, joan nahi içan cirenian plaçara jakiteko cer passatou cen, etçouten ikhoussi etcheric batere: etchen lekhian, bacen poutçou handi bat, egounco egunian oraïno khaoussitcen dena Barbazaneco plaçaren eta Saint-Bertrand-de-Comminges deitcen den hiriaren artian.

Behar dire lagoundou malouroussac.

\section{oOo}

Tantugou da guiçon çahar bat gordatcen dena oyhanetan. Çaintcen ditou elguiac eta peintciac amoreagatic nehorc esteçan han deus ebats. Noiz oguiac picatiac baitire edo belharra ebakia, hec gouciac naoussiac sarthou artian. Tantugouc estu loïc eguiten; iratçarria dago bethi. Haren aguertcen ikhousteco beldurrac daouzka ohognac. Houna da, sinhets eçacie, bena salbaya da. Laborari batec, çoïnac beitçakien hanitz gaouça, erraïten çakoten oussou bere semer Tantugou cela segourki lourreco fruitien çaintçaleric hoberena.

Ustailla hilabeteco arats aldi batez, ardi moultzo bat alha çagon bortou baten gagnian; artçagna lokhartu cen: iratçartcian, ohartou cen Tantugouri, çoin ihessi beitçouen oïhaneko aldeat. Içan cen guisa berian eçagoutia cabalaçain batçouez our ondo pouillit batian etçana arroca baten gaïnian.

Oraïno egounco egounian, Pyrénéetaco herri batçouetan, haurrac beldour dira harendaco. Noiz mouthico gnimigno batec edo nechcato gnimigno batec espeïtu obeditu nahi bere amari, hounec erraiten dako: Gogo emac! Oïhu eguiten dacoat Tantugouri, eta ichtantian jinen duc oïhanetic! 


\section{Barbazanen ichtorioua}

Bassoussoun léhénago Pyrénetaco herri batian, guissone bat éta émasté bat souïnac biciki saharac béitcién. Etcissién étché ttipi bat, baatcé bat éta béhi bat bécik. Béna, haïn praoubé izana gatic jokhorritcén citcien béthi héc béno praoubio ciénak. Egun batés lurra élhourrés coucoutia soussoune éta harrién érdiraastéco kharrougn bat éguitén cicine. Herrico yéndé gussiac bérotcén ari citsoune yatiaïkine éta édatiaïkine; abératx éta irousj citsoune. Ilhoune nabarriane, bi piaïyant souinaq héldou beitcirén ourhounic, nahi oukhane cicién baatu lékhou hartane cérén hots éta gojé beitcién; yo cicién bortha batian eta bejte batsoutane oono: néhoc étciticién outci nahi oukhane jartcéa, és déoujéré éman yatéco. Kajatiac ootaïc, chakhour fouriojés perjéitiac, bi éstranyérrék étciakicién noat yuan, noïs eta ere guérthatcén béitira hérriaine béjté bouriane, guissone eta émasté praoubé héén étchiaïn aïntciniana. Héén plégniac intçoutiaïkin, jenharra emann soussoune léihouane héér oïhou éguitéco, éta hainne émastia yuane soussoune fité borthaïn sabaltcea. Héér galdine gabé nor cién, jar araci citcién éta yar araci bérén jou bajterriane; guéo, hoc cerbitchatou ciessoune éjné eta soumbéit gastagna, soustén gucia. Béna ordiane, Gure Yaouna, - houa soussoune, Yon Doni Petriikine, - choutitou soussoune, bouria oro arguitia, eta errane ciessoune: "Ciek, praoubé eta houne cisté; cién aïssouac abératx éta gachtotçu. Yincoarén yujticia ina issane dailla!" Béhala lourra ikhaatou souçoune, Yéjouj éta Yon Doni Pétri itçaldou éta bi yéndé sahar horiéc émann bélhaounico. Bihaamoune goïciane, nahi oukhane souténiane herrirat youane yakiteco cér phajatou cén etcicién étchérïc bihi bat ikhouji: héén lékhiane, lac bat bassoussoune, houa béra souina béita oono égoune Barbazanéco hérriaïne éta Yon Doni Bettan Commingeseco hiriaine artiane. Béhartcié maléroujac laoundou.

J est mis pour $\underline{s}$; il se prononce comme le $\underline{s}$ espagnol.

\section{Tantugouïne ichtorioa}

Tantugou doussou guisson sahar bat souïna oïhanétan gordatcén beïta. Béiratcén tci alhorrac éta phéntciac, hétane ez dessatén déousjéré ebatx. Oguiac phicatiac diénian édo belharrac ébakia, oro naoujiac ideki artiane, Tantugou étci loï batéré itén: béthi beillatcén tci. Haïn aguértcen ikhoujtéco béldourrac atchikitcén tci ohognac. Houna oussou, jinhetx acie, béna jalbaya oussou. Biciki gaoussa sakién laborari batéc erréiten ciine béré jémér Tantugou céla jesurki lourreco froutén beïatçaleic hobenena.

Oustaillaco hilaiteco atxaldé batés, ardi tropa bat alhatcen çououne méndi batén gagniane; artcagna lokhartou soucoune: iatçartciaïkine aperceitou ciine Tantugou zouïnac ihés itén béitcién oïhaneco aldéat. Essaoutia issan sououn oorat béhi sagnés mendi arté pollit batiane, arroca batén gagniane etsana.

Oono égoungo égouniane, Pyrénéessétaco hérri batçutan, haourrac horrén béldurtçu. Mouthico édo néchkato ttipi batéc estiénian obéditou nahi béré amari, hunéc erréitén diacossou: Gouardimac hiri! oïhuïten diat Tantugouri, éta instantiane yinén douc oïhanetic!

Instituteur: Primorena, âgé de 27 ans, né à Ostabat Coll.: Etcheçahar, maire 


\section{Barbazaneco erran çaharra}

Bacen bestordous Pyreneetaco herribatian guiçon bat eta emastebat çoin beïtcien bissiki çahar. Etcien etche chipi bat, baatce bat eta behi bat baïci. Bena çoumbat nahi prauve cien, laguntcen çutien bethi houac beno prauviago cienac. Egunbatez lurra gordia cen elhurrez, harrien hausteco kharroua aïcen. Herrico gentiac oro beotcen cien jaten eta edaten cielaïc; abeax eta ious cien. Ulhuntcian bi bidejant jiten cienac hurrunti, nahiçan cien baatu lekhu hartan, ceren hotz eta gosse beïtcien. Yo cien bortha bat, gueo bestebat, eta beste çoumbait oano: ihourkere etçutin utci nahi sartcera, ez eta hayer eman jatera. Ohiltiac aldootaïc jarraïkiac chakhur coleatiez, bi arotcec etçakien nouat jouan, nouïs ere edien beïtcien herriaen basterrin, guiçon eta emaste prauve hayen etchen. Entçutez hayen plagniac, senhara jarri cen leyhouan hayen deytceco eta emastia jouan cen berthan borthaen çabalcera. Hayer galtheguin gabe nour cien, sareaci çutien eta jar eaci bere suthondouan; gueo cerbutchatu ceyen esne eta çoumbait gaztagna, cutienac oro. Bena ordin Goure Yaüna -houa eta Yondane Phetiri cien- jeyki cen haen buriac, arguitcen cialaric eta eran ceyen: "Ciec prauve cidie eta houn, cien aïçouac abeax dia eta gaïsto. Gincouaren justicia eguin bedi." Hain sarri lurra ikharatu cen, Jesus eta Yondane Phetiri gorde cien eta bi çaharrac belharrico eman cien. Bihamen goïçan jouan cienin herila jakitea cer igaran cen etcien ikhoussi etcheïc batere; hayen lekhian bacen ossin bat, çoin edieyten beita oano eygun Barbazaneco herriaren eta Yondane-Bertrand-Commingeco hiiaren artian. Behardia laguntu beharetan dienac.

\section{Tantugouen erran çaharra}

Tantugou da guiçon çahar bat gordatcen dena oyhanetan. Beguiatcentu alhorrac eta pinciac han deüsere ebax estecen. Oguiac edo belharra ebakidienian naüssiac oro bil artino, Tantugou esta batere lotcen; bethi iratçaria dago. Haren aguertcen ikhousteco loxac etchekitcentu ouhougnac. Houn da, sinhex acie, bena salvage da. Laboraribatec çougnec beïtçakin hanitch gaiça, erraiten cin ussu bere semer, Tantugou cela segur lurreco fruten beguiraçaleïc hobena.

Ustarilaco aristiribatez, ardi saldobat alhacen mendi baten gagnen; artçagna lothucen: iratçartciareki ikhoussicin Tantugou ihes eguiten oyhanerat. Içancen ere eçagutia artçagnez plano eger batin, etçanic harphegnabaten gagnen.

Orano eygun Pyreneetaco çoumbaï herritan haurrac haen loxa dia. Nouis ere mothico chipibatec edo nescato chipibatec espeytu nahi obeditu bere amari hounec errayten deo: "Abisa hadi! Deytcendiat behala Tantugou ginen duc (edo ginendun) behala oyhaneti!"

Instituteur: Pouy (Jean-Baptiste), 42 ans né à Arette (Basses-Pyrénées) Coll;: Aguer Jean 
Bertcé ordouz bacén, méndi horiétaco herri batian guiçon bat eta émazté bat biciki çahartiak. Ez çuten etche ttipi bat, baratcé bat éta béhi bat baïcik. Baïnan çonbat nahi praubé ciren, béthi lagountcén çouztén berak baïno praoubiagouak. Egun batéz, lourra élhourrez gordia cén éta kharroïn bat gaïtca bacén. Herriko yéndé gouciak soubazterrian cirén, yatén éta édatén, abérats eta irous. Ilhountciarékin, bi bidaïant, ourrounétik heldou cirénak, nahi ukhan çoutén guelditou lékhou hortan, cérén hotz éta gosé baitcirén; yoïten douté borta bati, bértcébati eta oraïno bértcé çonbaiiti: néhork éz ditou ouztén sartcéra, éta éz émaiten yatéra. Gouciétarik kasatiak, çakourraz yarraïkirik, bi éstranyérék éz çakiten norat yoan, noïz éta éré heltcén baïtira herri baztérréra, guiçon éta émazté praoubien étché aitcinéra. Horién plagnouak éntçoutiarékin, sénharra léïhora doua hékién déïtceko éta émaztia badoa fité bortharén çabaltéra. Galdétou gabé nor ciren, sarraraztén éta yarraraztén touzté sou ondoan. Guéro chérbitchatcén déé ésnia éta gaztainna çonbaï, çutén goucia. Baïnan ordian, Gouré Yincouak - houra cen Yondoni Pétiri ékin - choutitcen da bouria arras argui éta érraiten dée: "Cïek, ciék praoubiak éta hounac ciezté; cién aouçouak abérats éta gaïchto dira. Yinkouarén yusticia éguin dadiéla!" Béréhala lourra ikharatcén da, Yésus éta Yondoni Pétiri gordé ciren éta bi çaharrak belhaouniko erortcen dira. Biharamoun goïcian, yoan nahi oukhan çouténian herrira, yakitéko cér pasatu cen, ez çutén etché bakhar bat ikhousi; haren plaçan, bacén hour ithandi bat, égoun oraïno ikhoustén dena Barbazan hérriaren eta Saint Bertrand de Comminges hiriaren artian. Sokhorritou béhar dira malérousak.

La letre s se prononce comme en espagnol.

\section{Tantougouen istorioa}

Tantugou oïhanian gordatcén dén guiçon çahar bat da. Béguiratcen ditou alhorrak éta pintciak déousé éz déçaten ébats. Oguiak éta bélharra pikatiak dirénian, naousiak oro sartou artino, Tantugouk ézdou lorik éguitén, béthi beillatcén dou. Horrén ikoustiarén béldourrak, ohoïnak ourhountcén dira. Houna da, sinéts acié, bainan salbaya da. Gaouça biciki çakien laborari baték, erraiten cién béré sémeri, Tantugou guiçonik ségurréna éta lourréko frouitién béguiratcéko.

Ouztaillako atsaldé batez, aciénda saldo bat, olhan cén méndi batian; artçaina loak hartcén dou: iatçarciarekin, ikoustén dou Tantougou, oïhanérat éskapatzén. Bérdin éçagoutia içan cén, méndi çola batétako artçain çonbaïtez, étçanik harroka batén gaïnian.

Oraino égoun, hérri çombaiitétan, haourrak horrén béldour dira. Mouttiko ttipi édo néchkato ttipi baték éz douénian amari obéditou nahi, érraiten déé: goardia hiri! Tantougouri oïhou éguitén diat, éta béréhala yinén douk oïhanétik.

La lettre $\underline{s}$ se prononce comme en espagnol. Instituteur: Elissagaray, 52 ans, né à S.Jean- Pied de Port Coll.: MM. Sarhy, Ganderatz 


\section{Barbazanco istoria}

Bazen lehenago Pyrenées taco herri batian gizon bat emaste bat biciki zaharrac. Ez zuten etche ttipi bat, baratze bat eta behi bat baïcic. Bena haïn pobre izanican ere sokhorritsen zusten bethi hec beno pobriago zienac. Egun batez lurra elhurrez cucutia zen eta kharrugna ari zen harrien erdiratceco gizan.

Herrico yende guciac berotsen ari ciren yatian eta edatian: aberats eta irus ciren. Ilhuntzin bi pideyantec, çoinac urrundic heldu baïtciren, nahi izan zuten guelditu, toki hartan, zendaco eta hotz baitciren, eta gosetiac, yo zuten bortha batian, guero beste batian ta beste frangotan; nehorc ez citusten nahi ukhan utzi sartzera, ez eta ere deusic eman yatera. Orotaric casatiac, chakhurrez segitiac, bi estranyerrac ez zakiten noat yuan, noïs eta ere khausitu baïtciren herriaren basterrian gizon eta emaste pobre hen etche aïzinian.

Hen plegniac entzunic zenharra yarri zen leihuan her oỉhu egiteco eta emastia yuan zen fite bortharen zabaltzéra.

Galdegin gabe nor ciren sar arasi citusten eta yar arasi su basterrian; guero eman zeen yatea esne eta gastegna cembait; zuten gucia.

Bena ordian gure Jainkoa -hura zen Yondane Petriekin- chutitu zen buria argi argia eta erran zeen:

"Ciec pobre ciste eta hun; cien aïzuac aberats eta gaïstuac dira. Yainkoaren yusticia egina izan dadiela." Ordu berian lurra ikharatu zen, Yesus eta Yondane Petri galdu ciren eta bi zenhar emaste zaharrac eman ziren belhaünica.

Biharamen goïzian, noïs eta ere yuan nahi izan baitzuten elizatherat, yakiteco cer passatu zen, ez zuten ikhussi etche bat ere, hen lekhuan bazien phutzu handi bat zoin khausitsen baita oraino egun Barbazaneco herriaren eta Saint-Bertrand-de-Commingeco hiriaren artian.

Lagundu behar dira pobriac.

Nota: $u$ se prononce ou

\section{Tantugouen istoria}

Tantugou da gizon zahar bat gordatzen dena oyhanetan: zaïntzen bitu alhorrac eta phensiac deusic ebatz ez dezaten. Nois eta ere ogiac mostiac baitire edo belharra ebakia, hec denac nausiac sar artio, Tantugou ez da loc hartzen: guardian dago bethi. Haren aguertzen ikhusteco beldurrac atchikitzen ditu uhugnac.

Huna da, sinhets zazié bena zalbaya da. 
Laborari batec, zoïnec beizakien biciki gaüza erten cien uzu bere semer Tantugou zela zegurki lurreko frutin zaïn zale hobena.

Ustarilaco atzalde batez azinda multzo bat alhan zagon bortu batian, arzaina loc hartu zen: iazartzian apercebitu zien Tantugou ezkapatzen zela oyhanerat burus...

Gisa berian ezagutia izan zen beste arzaïnes etzanic phegna batian.

Oraino egun, Pyrénées taco herri batzutan haurrac haren beldur dira.

Nois eta ere muthico ttipi batec edo nescato ttipi batec ez baitu obeditzen bere amari, hunec erten dio:

"Guardia emac! Oỉhu egiten doat Tantugouri eta istantian ynen duc oyhanetic."

Instituteur: Lapeyre, né à Charritte-de-Bas 


\section{Barbazanen istoïa}

Baçucun léhen, Pyrénées deicen den lekhubatetan, guiçon éta émaste bat, hanich caharrac. Etcicien etche thipibat, bâcebat, éta behibat baïcic. Haléican éé, laguncen citicien, bérac beno praubé ago ciénac. Egun batés, elhurra çuçun éta carronté handy. Hanco gentiac oro béotcen jaten, éta édaten aiçûtutcen; abéatz éta ious cütutcen. Ulhun tcébatez, bi bijéjant hurruntic houna ciénac nahi içan çutucen ukhuatu léqu hartan, hostuic éta goshétuiric béiciren: bortha hanits jo cicien, ihour quec écititcien éisi sarcera, es eman jatera. Otaïc igorric, tçaqur gaïtz élibat ondotic, bi arrotsec etçaquicien nouat jouan, nous éé édien beicien carricaco asquen etchetan guiçon eta émaste praubé houac égoïten ciéna. Plagnien ençutiaéquin, senharra jouan çuçun leïhoula déitcera éta émastia fité bortharen çabalcea. Batéé galthatu nour cien! sar éaci citicien éta supaster tchocouan jar éaci, guéro éman ciéçun ciénétaìc: esné éta gastagna çouinbat. Ordian Ginco Houna - ceren houa çuçun Jondane Pethiry éré jeĩqui çuçun, buia arguibates ungüaturic eta erran céen: "Praubé sidé éta houn, bestiac abéatz, éta gaiisto. Justicia éguinic içan dadila". Ber mémentouan lurra iqharatu cen, Ginco Houna eta Jondane Phétiry hantic galdu, etea bi chaharrac belhaïco éoï. Bihamenian carricaï soguin ciénian, isla handibat cen haen léquian, Barbazanen éta St Bd Comminge artian.

Soccorri behar dicié maléusec.

\section{Tantugouen istoïa}

Tantugou duçu guison chaharbat oyhanpétan gordatcen dena. Béguiacen titçu alhorrac eta sohouac déusic ébats estécen. Nouis éré oguiac piquaturic edo belharrac daillaturic beitira, Tantugouc béguiatcen titçu béthi, naousiac sarartino. Ouhougnac haen ikhousteco loçac béguiacen titçu. Houn duçu, bena salbage. Hanich çaquian laboaï batec béé semer erraiten çiçun fruten béguiaçaleic hobena aïsa Tantugou cela.

Ustaïlaco arrasti bates, ardi tropabat alhan aïçuçun mendian; artçagna lothu çuçun, iratçarciaequin ikhousi çiçun Tantugou oyanila buüs escapatcen. Hala hala, ibar ejerbatétan cien artçagnec éçagutu cicien botchu batetan etçanic.

Oano, günecal, haurrac lotça tutçu haen: nouis éré mouthikho édo nescatila thipibatec espeïtu sinhési nahi ama, erraiten dioçu: Abisadi! Tantugou déicent diat, éta béhala jien duc oyhanétic. 
Observations sur la prononciation. $\underline{e}$ est toujours ouvert $-\underline{z}$ se prononce comme l's français. - L's se prononce en appuyant la partie antérieure de la langue contre le palais et en laissant filer le son.

Bazuzun, lehenago, Pyrienoetaco heri batetan, guizoun eta emaztebat hanitch zahar beitziren. Etzizien etche tchipibat, baratzebat eta behibat baïzik. Bena zoumbat nahi praoube ziren, sokhorritzen zitizien bethi hourak beno praubiago zirenak. Egun batez elhurez chouri zuzun, eta kharountatzen zizun harien erdiratzeko hegniala. Jentiak oro berotzen zututzun jatiaeki eta edatiaeki; aberats eta ious zututzun. Ulhungnabartzian bi bidejant huruntik houna zirenak nahi izan zutuzun ukhuratu lekhu hartan, hotz eta gose beitziren. Jo zizien bortha bat, guero bestebat eta beste hanitch orano. Ihourkere etzititzien sartzera utzi nahi ukhen ez jatea deüs eman. Ohilturik orotarik, lasterkaturik tchakhur gaïtzez, bi estranjerek etzakizien nouat jouan ediren zienian heri bazterian, guizoun eta emazte praube hen etche aitzinian.

Haïen plagnien entzutiareki, senhara leïhoualatu zuzun hen deïtzeko eta fite bortharen zabaltzeratu haren emaztia. Nour ziren galthatu gabe, sar eta jar erazi zitizien suthondouan; guero zerbutchatu ziezun ezne eta gaztagna zoumbait; ben hountarzun oro. Bena ordian, Goure Jauna - houra zuzun Joundane Phetieki- tchutitu zuzun buria argui arguia eta eran ziezun.

"Ziek praube et houn zide, zien aïzouak aberats eta gaïchto, Jincouaen justicia eguin bedi". Haïbestenaeki lurra ikhaatu zuzun, Jesus eta Joundane Phetiri galdu eta bi zaharrak belhaiko eoï. Bihamen goïzian heriala jouan nahi izan zienian jakiteco zer igan zen, etzizien ikhousi etcherik bate. Hen lekhian bazuzun putzu handibat, egun uano edireiten dena Barbazaneko heriaen eta SaintBertrand-de-Comminges hiriaen artian.

Behar dizie laguntu praoubek.

\section{oOo}

Tantugou guizoun zaharbat duzu gordatzen dena oïhanetan. Alhorrak eta sohouak beguiatzen tizu ihourkere deüse han ebats eztezan. Oguiak phikatuik edo belhara daillaturik dienian buruzaguiaz oro bildurik izan artino, Tantugouk loïk eztizu eguiten; beillatzen dizu bethi. Haen aguertzen ikhousteko lotsak ekhuazten tizu ouhougnak. Houn duzu, sinhets ezazie, bena basa. Laboaï gaïza hanitx zakin batek, eraïten zizun usu bere semer Tantugou zela segurki lureko usten beguirazaleik hobena.

Uztaïlako eguerdi ondo batez, ardi saldobat alhan zuzun borthian; artzagna louak hartuzizun: iatzartzian, ikhousi zizun Tantugou ihes eguiten oïhanialat buruz. Izan zuzun hala hala ezaguturik behizagnez naba ejerbatetan harokan gagnen etzanik.

Oano egun, Pyrineoetako zoumbait heritan, haourak haen lotsa tutzu. Mothiko edo neskatila tchipi batek sinhetzi nahi eztianian bere ama, hounek eraïten diozu: Abisadi! Deïtzen diat Tantugou eta, behala jinen duk oihanetik! 
Baçututçün lehenago Pyrénétaco heri batetan guiçon bat eta emasté bat çougn hanitch çahar beitçien. Etcisiën etche tchipi bat, baatsé bat eta behi bat baïcic. Bena çoumbat nahi praubé ciën, sokhoritsén citiciën houac beno praoubiago cienac. Egün bates, lura elhurés gordeïc çucün eta kharountatcen cicün hariën erdiatseco hégnïn. Hérico jëntic oro, beotsén çututçün jatiéqui eta édatiéqui; abéats çutuçün eta ious. Ulhüntsian, bi voyajur, çougn hora beitsiën hurüntic nahi içan çutuçün arestatu lekhu hartan: ihourquec etsitiçün nahi ükhen utsi sartsera, ez nahi ukhën eman deüsé jatéa. Casatüic aldi orotaïc, perséguituïc chakhur gaïts eli bates, bi estranjéec etsaquiciën nouat jouan, nouïs eta éré heltu beitsiën, heri basterïn guiçon eta emasté praoubé hayën etché aïtziniala. Hayën plagniën ëntsutiéqui, sënharra jalkhi çuçun leyhouala hayer oyhu eguitéco eta emastia jouan çuçün fité borthaén sabaltséa. Galthatu gabétaïc, nour cién, sar eraci citiciën eta jaraci suthondoun, guéo cerbutchatu çieçün esné eta gastégna çoumbaï, hec çutuçün çütienac oro. Bena ordïn, gouré Jïnco houna, - houa çuçün Sën Piéraéqui- jeiqui çuçün buia argui arguia eta eran çieçün: "Ciec praubé cidié eta houn; cien aïcouac abéats tüçu eta gaïchto. Jincouaïn justicia eguin bédi!"

Haïmbestenaéqui, lura ikhaatu çuçün, Jésus eta Sën Piera galdu çutuçün eta bi saharac belhaïco éoi çutuün... Bihamën goïcian, nahi içan cienin jouan hirila, jaquitéco cer igan cën, etsicien ikhousi etchéic baté: hayën lékhïn, baçuçün losco bat, Barbazan deitcën dën hériën eta Saint Bertrand de Comminges deitcën dën hiiën artïn egün dena.

Béhar dicié maléousec lagüntu.

\section{oOo}

Tantougou oyhanétan gordatcën dën guiçon çahar bat duçu. Béguiatcën tiçu alhorac eta sohouac, han deüsé ébax estécën. Oguiac phicatuic diénïn edo belhara ébakiic, buçaguiac oro bil artino, Tantugou estuçu baté lotsën; bethi beillatsën duçu. Haïn aguertcën ikhoustéco loxac érétinitsën tiçu ouhougnac. Houn duçu, sïnhex éçacié, bena basa duçu. Gaïça hanitch saquïn laboaï batec eraïten ciéçun béré sémer lureco frutën béguiaiic hobéna cela ségur Tantugou.

Ustaïlaco arasti batez, ardi tropa bat alha cuçün bortian, artsagna lothü çuçün: iratsartsiequilan, apercebitu ciçün Tantugou çougn escapatsën beïtsën oyhanialat. Içan çuçuün orano eçaguturic ardi béguiaï eli bates naba batetan, etsanic aroca batetan gagnën.

Oano egün, Pyrénéeco heri eli batetan, haürac loxa tuçu haïn. Mothico tchipi edo neskatchou batec estinïn nahi sïnhexi béré ama, eraïten dioçu amac: Abisa hadi! Oyhéguitën doat Tantugoui, eta mémëntin jinën duc oyhanéti!

Etchecopar, 26 ans, né à Moncayolle Coll.: Etchebarne Adolphe 


\section{Barbasaneco érantchoua}

Baçucun léhénago Pyréneetaco héri batétan guiçoun bat éta émastebat hanitch çahar ciénac. Etcicien etché tchipi bat, baratce bat éta béhi bat baïcic. Béna coumbat nahi praoube cien béthi sokhoritcen citicien béac béno praoubiagouac. Egun batez lura élhurez gordëic çuçun éta hariac e kharountatcen. Hérico jentiac oro béotsen çaoudetçun jatez éta édatez: abéats éta ious çutuçun. Ulhuntcian bi bidajant hurundic houna cienac nahi içan çutuçun ukhuatu lékhu hartan gosé eta hotz icanez. jo cicien bortha bat, guéo beste bat, guéo beste coumbait oano. Ihourc ée etciticun nahi ukhen utzi sartcea, ez éta ée éman jatéa. Igoriic ootaïc, jaraïkiik tchacur gaïztiez, bi arotcec etçakizien nouat jouan nouiz éta ée édien béitcutien bée buiac heri baztérian guicoun émazte praoubé haien etche aïtcinian. Haien plagniac entçunic senhara jari çuçun leïhouan haien deitceco éta émaztia jouan çucun borthan idekitcea. Galthatu gabe nour cien sar éaci éta jar éaci citicien suthondouan: guéo éman ciecun ezne éta gaztagna coumbait: ciena oro. Béna ordian Goue-Jauna - houa çuçun Joundane Phetiiekijaïki çuçun buia distiant éta éran cieçun: Ciec praoube éta houn ciade cien aïcouac abéats éta gachto tuçu. Jincouaen gaztiguia éguinic içan dadiala: Hagn sari lura ikhaatu çucun Jesus éta Joundane Pethiri galdu çutuçun eta bi çaharac éoï belhaïco. Bihamenian nahi ican cienian jouan hérialat jakitéco cer heltu cen etciticien ikhousi etche bat ée: haien lekian baçuçun islaca bat, égun oano édieïten déna Barbasaneco hériaen éta Joundane-Betran-Commingeco hiiaen artian. Praoubec béhar dicie sokhoritu.

\section{Tantugouen érantchoua}

Tantugou oihaénétan gordatcen den çahar duçu. Béguiatcentiçu alhorac éta sohouac deüs han ébastetic. Oguiac ébakiic dienian édo belhara daillatuic agniketa oro buuçaguiaz bilduik içan artio. Tantugou estuçu lotcen batéé. Bethi beillan diagoçu. Haen ikhous lotsac guibeltsen tiçu ouhougnac. Houn duçu, sinhets acie, bena basa duçu. Laboai jakintsu batek éraïtencisun usu bée semer Tantugou cela segurki luréco frutien béguiaçaleic hobena.

Ustaillaco aristi batezn ardi saldo bat alha çuçun mendian. Artçagna lothu çuçun iatcarciareki ikhousi ciçun Tantugou oïanialat buuz escapatcen. Berguisan éçagutuik içan çuçun artçagnez naba eijer batetan etçanic botchu baten gagnen.

Egun ée oano Pyréneetaco héri coumbaïtetan haourac haen lotsa tuçu. Mothico édo nescatila tchipi batec eztianian ama sinhetsi hounec éraiten dioçu: Déitcen diat Tantugou éta behala jinen duc oïhanetic

Instituteur: Abadie, 27 ans, Urrugne Coll.: M Lililbéhéty, Mendy 
Bacén besté ordus Pyrénétaco héri batetan guiçoun bat eta emasté bat çoïn icigari çahar beitcien. Etcién étché tchipi bat baïcic, batcé bat eta behi bat. Bena, çoumbaït nahi praübé cien, sokhoritcen çutien bethi houac çouïn praübe (pra-oube)ago beittcien béac béno. Egun batés, (sonner l's), lura gordeïc cén elhurés, eta kharou éguiten ciann haria erdiartino. Hérico jéntiac oro béotcén cién, jatiaëki eta édatiaeki; abéatx cien eta ious (s sifflante). Ulhuntcian, bi bidéjant, çougn hurruntic jitén beïtcien, nahi içan cien ukhuatu lekhu hartann, cén eta hots beïtcién eta gosé (s sifflante); jo cien bortha bat, guéo besté bat eta besteĩc, (s sifflante) oano: ihourc é étçutien nahi ukhén sartcéa (s sifflante) utci, ez éta deüzé (z sifflante) émann jatéa. Ara igoric otaiic, jarraïkic tchakur fuiouzés, bi estranjec étçakién noua jouann, nouis é édién beïtcién hériaïn bastériann, guiçun éta émasté praübé cién hén étchiaën aïtciniann. Entçutiaïki hén plagniac, senhara jarri cén leïhouala hén deïtceko, éta haën émastia jouan cén bertann borthaën çabaltcéa. Her galthatu gabé nour cién saraci (s sifflante) çutién eta jaraci hén suthondouann; guéo cerbutchatu cén ezné eta gastagna çoumbait, ciéna $\mathrm{O}^{2}$. Bena ordian Jinco (Dieu) Goue Jaüna (Ja-ouna) -houa cén, Joundané Phétiëki (prononcer p et h) -jeiki cén, buia argui arguia éta erran cén: "Ciec, praübe cidé eta houn; cién aïçouac abéatx diadé eta gachto. Jincouaën justicia (s sifflante) éguinn dadinn! Haïnn sarri lurra ikhatu cén, Jésus (les deux sifflantes) eta Joundané Phéti galdu cién eta bi çaharrac éoy cién belhaïco. Bihamén goïçann, nouis é nahi içann beitcién jouann heriala, jakitéco cer (faire sonner l'r), igann cén, etcien ikhousi (s sifflante) étché bat é: hén lekhiann bacén hour handi bat, houa çougn édieïtén beita oano égun. Barbazaneco heriaën eta Sén (sifflante) Bertrann-Cominjaco hiiaren artiann. Behar da laguntu maléouzac ( $z$ sifflante)

\section{Tantugouen ichtoïa}

Tantugou guiçoun çahar bat da çoin gordatcén beïta oyhanétan. Béguiàtcén dutu alhôrrac eta sohouac (faire siffler l's) han ébatx estécén deüzé. Nouis é oguiac moustuic béitia edo belhara thaillatuic, buçaguias içan artino oro eretiatuic, Tantugou ezta baté lotcén; cazu eguitén du bethi. Haën aguertcén ikhoustiaën loxac éréténitcen dutu ouhougnac.

Houn da, sinhex (prononcer si-ne-hex - s sifflante) éçacié; béna salbajé da. Laboaï batec çougnec beïtçakian gaïça hanitch eraïten cian usu (s sifflante) bé sémer (faire sonner l'r) Tantugou céla segurki lurreco frutien béguiaçaleïc hobéna.

Ustaila hilabétéco arasti (s sifflante) batés (s douce) ardi saldo bat (s sifflante) alha aïcén méndi batétan (ta-nn) gagnén. Artçagna lothu cen. Iatçartciaiki, apercebitu cian Tantugou, çougnec ihès (s sifflante) éguitén beïtcian oyhanialat bus (prononcer l's). Içan cén é éçagutuic artçagnès (prononcer l's) arbailla ${ }^{3}$ éjer batétan, étçanic botchu batetan gagnén.

2. Ou oro, tout

3. Ou ibar, valllée 
192

Oano égun, Pyrénétaco héri çounbaitetan (prononcer l'n) haurac (ha-ou) haïn loxa dia. Nouis é mothico tchipi batec edo nescatila tchipi batec espeitu nahi obéditu bé amaï, erraïten déo: Abizadi, ou beguiadi! deïtcen diat Tantugou eta ichtantian oyhanétic jinen da.

Quihillalt, 28 ans, né à Ordiarp, près Mauléon Laffargue, 56 ans, nè à Haux 


\section{Barbazaneco aleguia}

Baçutuçun lehenago herri batetan guiçon bat eta emazte bat icigarri çahar. Etcicien etche ttipi bat, baatce bat eta behi bat baïcik. Bena çoumbat nahi praoube içanic ere, sokhorritcen citicien bethi beac beno praoubiago cienak. Egun batez lurra elhurrez gordeïk çuçun eta kharroua ari çuçun harrien erdiatceko guisan. Herriko jentiak oro ari çutuçun berotcen, jaten eta edaten cielaik; abeats eta irous çutuçun. Ulhuntcian, bi bidajant, hurruntik jiten cienak nahi içan çutuçun baratu lekhu hartan, hoztuic eta gosetuik beitciren; jo cicien bortha bat, guero beste bat eta beste elibat orano: ihourk ere etçititçun nahi ukhen utci sartcera, ez eta deusere eman nahiukhen jatera. Orotaik igorriric, chakhurrak gaizturik ondotik, bi estrandjerek etçakizien nourat jouan, nouiz eta ere aguitu beitcien herriaren bazterrin guiçon eta emazte praube ciren haien etchiaren aitzinin. Haien plagnien entzutiarekin senharra jarri çuçun leihoun hen deitceko, eta emaztia joun çuçun çalhe bortharen çabaltcera. Batere galthatu gabe nour ciren, sarreraci citicien eta jarreraci suthondoun; guero cerbutchatu cieçun ezne eta gaztagna çoumbait, çutienac oro. Bena ordian, Goure Jaouna - houra çuçun Joundane Phetirirekin - jeiki çuçun, buria argui arguia, eta erran cieçun: "Ciec, praoube eta houn cide; cien aiçouc aberats eta gachto tuçu. Jinkouaren justicia eguin dadila!” Hagn sarri lurra ikharatu çuçun, Jesus eta Joundane Phetiri galdu çutuçun eta bi çaharrak belhaiko erori çutuçun... Biharamen goiçan, nahi içan cienin joun herrila, jakitera cer igaran cen, etcicien etcheric batere ikhousi: hen lekhin, baçuçun losko bat, orano egun Barbazaneko herriaren eta Saint Bertrand de Commingeko hiriaren artin edireiten dena. Behar dicie malerousek laguntu.

\section{Tartazen aleguia}

Tartas guiçon çahar bat duçu gordatcen dena oïhanetan. Beguiatcen tiçu alhorrac eta sohouac deusere eztecen hantic ebatx. Oguiac mouztuic dienian edo belharra ebakiric, oro buruçaguiac sarthu artio, Tartas eztuçu batere lotcen; bethi kasu eguiten dizu. Haren aguertcen ikhousteco lotsac ouhouignac egoneazten tiçu. Houn duçu, segur içan cite, bena basa duçu. Hanitch gaiça çakian laborari batec erraiten cieçun usu bere semer Tartas cela segurki lurreco frutien beguirariric hobena.

Uztaïlaco arrestiri batez ardi saldo bat alha çuçun bortu batetan, artçagna lothu çuçun: iratçartciarekin ikhousi çiçun Tartas oïhanialat ezkapatzen. Hala hala eçaguturic izan çuçun artçagnez ibar ejer batetan, botchu batetan gagnen etçanik.

Orano egun, Pyreneetaco herri çoumbaitetan, haurrac haren lotsa tuçu. Mothico ttipi batec edo urruchouna ttipi batec eztianian bere ama sinhetsi nahi, erraïten dioçu: Abiseik! deitcen diat Tartas, eta, mementin, jinen duc oihanetik.

Instituteur: J Erréçaret, âgé de 26 ans, né à Sauguis (canton de Tardets) Coll.: Onnaïnty Jean, Jaurégiber Pieri 


\section{Barbanzaneco historia}

Bacen lehen ago Pyrenéetako herri batetan guiçoun bat eta emazte bat icigarri saharturic. Etsien etche tipi bat baïcic, baatsé bat eta béhi bat. Bena çoumbat nahi praoubé issanic sokhoritcen sutien bethi berac beno praubiagouac. Egun bates lurra thapaturic çuçun elhurez eta kharoua éguiten ciçun haria erdiratuco beitcian. Herrico jentiac oro bérotsen ai sien, jaten eta edaten sielarik, oro aberats sien eta irouz. Ulhuntsian, bi bidajant huruntic jiten cienac, nahi issan sien ukhuatu han seren eta hots beitsien etaé gossé; bortha bat trucatu sien, beriz beztebat eta besteïc éré orano; ihourc éré etsutian nahi ukhen utsi sartsera, ez eta éman déuz éré jatera. Ohilturic orotaric, kharicaco hoez jarraïkiric, bi arotsek etsakien nouat jouan, nouiz eta éré heltu beïtsien heriaren basteriala guisson haen eta emazte çahar haen etchiaen khantiala çougn praoube beïtsien. Hen phenac ikhoussiric eta hen plagniac ensunic, senhara leïhouala aguertu sen, her oïhu éguiteco eta emaztia fite jouan sen borthaen sabalsera. Galthatu gabetaric nour sien sar éassi sutien eta jar éassi béré suthondouan; Guero serbutchatu seren ezné eta gaztagna çoumbaït sutien gaïssetaric. Bena ordian goure Jinco Jaouna - houa sen Joundané Phétirieki,- jeiki sen buria béré brilllantsia oroekin, eta erran seren: "Siec praoube siade, bena houn; sien aïssouac aberats dira eta gaïsto. Jinkouaren justissia éguin dadila". Ber mementouan lurra ikhaatu zen, Jesus eta Joundané galdu sien eta bi çaharac belhaïcatu sien... Biharamen goissian, nouiz et éré jouan nahi issan beitsiren heriala jakitera ser igan zen etsien ikhoussi etche bat éré, hen plassan, bassen hour ukhuatu bat çougn edireiten béita oano Barbazaneco heriaren eta Saint-Bertrand- de Commingeco hiriaren artian.

Behar dissu bethi éguin kaitatia praoubiaï.

\section{Tantugouen éran çahara}

Tantugou çahar bat da çougn gordatsen béita oïhanetan. Béguiatsen tu alhorac eta sorhouac ébats estesssen deuzéré. Oguiac ébakirik dienian edo belharac burussaguiac eaman eartekan, Tantugou esta lotsen, gouaïta bethi. Tantugou ikhous beldurez ouhougna béguiatsen da ébastetic. Houn da, sinhets essassu, bena bassa da. Hanich çakian laborari batek eraïten sireçun béré semer segurki Tantugou sela lurreco fruten béguiraçalerik hobena.

Ustailaco ariztiibatez, ardi saldo bat alha sen bortu batetan, artsagna louac hartu sin; iatsartcian ikhousi sian Tantugou oïhanialat jouaïten. Ber guizan essaguturic issan sen ulhagnez ibar éijer batetan, etsanic har botchu batetan gagnen.

Orano égun Pyrénéetako çoumbait heritan, haurac lotsa tuçu harentaco. Nouiz eta éré mothico tipi batec edo urutchouna batec espeitu nahi sinhexi bere amari, harec éraïten diroçu: Abisadi hi! deitçen diat Tantugou eta bertan jinen duc oïhanetic! 
Baçuçun lehenago Pyreneetaco herri batetan, guiçon bat eta emaste bat icigarri çahartiac. Etcicien beste hountarçounic, etche tchipi bat, baratce bat eta behi bat baiciq. Bena çombat nahi prauve cien, sokhorritcen cititcien bethi berac beno prauveagouac. Egun batez, lurra elhurrez gorderic çuçun eta kharrou icigarri handi. Herrico gentiac oro berotcen çutuçun jaten eta edaten ari cielaric; aberats çutuçun eta irouz. Ulhuntcian, bi bidejent hurrunti horra cienac, nahiçan çutuçun ekhuatu lekhu hartan, hotz ta gosse beitcien; jo cicien bortha bat, guero beste bat eta guero beste çombait: ihourc ere etcititcien nahiçan sartcera utci, ez eta deuz eman jatera. Orotaric ohilturic, hor gaitz eli bat ondouan, bi estranjerrrec etçakicien nourat jouan, nouiz ere herri basterian aguitu beitciren, guiçon eta emaste prauve hayen etchaitcinian. Hayen plagniac entçunic, senharra leyhoula jouan çuçun hayen deitcera, eta emastia fitez bortharen çabalcera. Galthatu gabe nour ciren, sar eraci citicien eta suthundouan jar eraci, guero cerbutchatu cieçun ezné eta gastagna çombait. Bena ordian, Jésus-Christ goure Jauna (houra çuçun Joundane Phetirieki) jeïki çuçun, buria arguituric, eta erran cieçun: "Cieq, prauve cide eta houn; cien ayçouac aberats tuçu eta gaisto, Jincouaren justicia eguinic içan dadila!" Behala lurra ikharatu çuçun, Jésus eta Joundane Pethiri galdu çutuçun eta bi çaharrac belharricatu çutuçun... biheramen goiçan, jouan nahiçan cirenian herriala, jakitera cer igaran cen, etcicien ikhoussi etchebat ere; hen lekhian, istil handit bat çuçun, orano orai Barbazaneco herriaren eta San-Bertrand-de-Commingeco hiriaren artian edireyten den ber istilla. - Behar dira laguntu malerousac.

\section{oOo}

Tantugou duçu guiçon çahar bat oyhanétan gordatcen dena. Beguiratcen tiçu alhorrac eta sorhouac amourecatic ihourc estacen deuz ere ebats. Oguiac phicaturik dienian eta belharrac thaillaturic buruçaguiac oro bil artino, Tantugou eztuçu batere lotcen; bethi beillatcen diçu. Haren aguertcen ikhousteco lotxac ouhouinak beguiratcen tiçu. Houn duçu, sinhets eçacie, bena bassa duçu. Laborari batec beitçakien hanitz gaiça, erraiten ciçun ussu bere semer Tantugou cela segur lurreco frutien beguiraçaleric hobena.

Ustarilaco eguerdi ondo batez, ardi saldo bat alha çuçun mendi batetan; artçagna lothu çuçun: iratcarciarequi, ikhoussi ciçun Tantugou escapatcen oyhanilat. Hala hala éçaguturic içan çuçun artçagnez naba ejer batetan, botche batetan gagnen etçanic.

Orano egunco egunian, Pyréneetaco herri çoimbatetan, haurac lotxa tuçu haren. Mouthiko tchipi bat, edo nechkatila tchipi batec estirenian nahi obeditu nahi amari, hounec erraiten diroçu: Abisadi oyhuguiten dignat Tantugouri, eta behala jinen dun oihanetic.

Iriart, 46 ans né à Larrau Coll.: Carricaburu Pierre 


\section{Barbasaneco erantchoua}

Baçuçun lehenago, Pyrénéétaco heri batetan, guiçoun bat eta emazte bat, hanitch çahar cienac. Etcicien etche tchipi bat, baatce bat eta behi bat baïcic. Bena, çoumbat nahi praoubé cien, bethi sokhoritcen citicien beac beno praoubiagouac. Egun batez, lura elhurez gordëic çuçun, eta hariac ee kharountatcen.

Herico jentiac oro beotcen çaoudetçun, jatez eta edatez: abeats eta ious çutuçun. Ulhuntcian, bi bidajant, huruntic houna cienac, nahi içan çutuçun ukhuatu lekhu hartan, gose eta hotz içanez. Jo cicien bortha bat, gueo beste bat, gueo beste çoumbait oano: ihourc ee etcititçun nahi ukhen utci sartcea, eta eta ee eman jatea. Igorriic ootaïc, jaraïkiic tchakur gaïztiez, bi arotcek etçakicien nouat jouan, nouiz eta ee edien beïtçutien bee buiac heri bazterian, guiçoun emazte praoube haïen etche aitcinian. Haïen plagniac entçunic, senhara jari çuçun leïhouan, haïen deïtceco, eta emaztia jouan çuçun borthaen idekitcea. Galthatu gabe nour cien, sar eaci eta jar eaci citicien suthondouan; gueo eman cieçun ezne eta gaztagna çoumbait: ciena oro. Bena ordian, Goue-Jaouna - houa çuçun Joundane Phetiieki - jaïki çuçun, buia distiant, eta eran cieçun: "Ciec, praoube eta houn ciade; cien aiçouac, abeats eta gachto tuçu. Jincouaen gaztiguia eguinic içan dadiala!" Hagn sari, lura ikhaatu çuçun, Jesus eta Joundane Phetiri galdu çutuçun, eta bi çaharac eoï belhaïco.

... Bihamenian, nahi içan cienian jouan herialat, jakiteco cer heltu cen, etciticien ikhousi etche bat ee; haien lekhian baçuçun islaca bat, egun oano edieiten dena Barbasaneco hériaen eta Joundane-Betran-Commingeco hiiaen artian.

Praoubec behar dicie sokhoritu.

\section{Tantugouen erantchoua}

Tantugou, oïhanetan gordatcen den çahar bat duçu. Béguiatcen tiçu alhorac eta sohouac, deüs han ebastetic. Oguiac ebakiic dienian edo belhara daillatuic, agniketa oro buuçaguiaz bilduic içan artio, Tantugou eztuçu lotcen batee. Bethi beillan diagoçu. Haen ikhous lotsac guibeltcen tiçu ouhougnac. Houn duçu, sinhets acie, bena basa duçu. Laboai jakintsu batec eraiten ciçun usu bee semer, Tantugou cela segurki lureco frutien beguicaleïc hobena.

Uztaïlaco aristi batez, ardi saldo bat alha çuçun mendian. Artçagna lothu çuçun, iatçartciaeki, ikhousi ciçun Tantugou oyhanialat buuz ezcapatcen. Ber guisan eçagutuic içan çuçun artçagnez, naba eijer batetan, etçanic botchu baten gagnen.

Egun ee oano, Pyrénéétaco heri çoumbaïtetan, haourac haen lotsa tuçu. Mothico edo nescatila thipi batec, eztianian nahi ama sinhetsi, hounec eraiten dioçu: "Abisaïc! Deitcen diat Tantugou, eta behala jinen duc oïhanetic!"

Hastoy, 35 ans, né à Alos-Sibas

Coll.: Etchecopar, Paul; Goyheneix, Marc 


\section{Barbazaneco ichtorioa çahara}

Bacien lehenago, Espagnalaco bortiari çagouen heri batetan, guiçoun bat eta emaste çouin hanitch çahar beitzien. Etzien etchetto bat, baratze bat eta behi bat baicic. Bena, çoumbat nahi praoube içana gatic, sokhorritzen çutien bethi berac beno praoube ago cienac. Egun batez, elhurrez gorderic cen lurra, eta kharou aidiac nahi çutian erdiatu harriac. Herrico jentiac oro berotzen ari cien jaten eta edaten ari cielaric; aberax cien eta irous. Ulhunaren heltziari, bi bidejente çouin hurunti houna beitzien nahi içan cien ekhuatu gune hartan, ceren eta hotz eta gose beitzien; jo cien bortha bat, guero bestebat eta beste çoumbait ere orano: ihourc ere etzutian nahi ukhen utzi sartzera, ez etzen eman nahi ukhen deusere jatera. Orotan guibel igoriric, tchacurac hen oundotik gaisturic, bi arotzec etzaquien nouat jouan, nouiz ere aguitu beitzien kharrika bazterian, praoube cielaco guiçoun eta emaste hen etche aitziniala. Entzutiarequi hen plagnien, senhara leihouala jelkhi cen haien deitzeco, eta haren emastia jouan cen çalhe bortharen çabaltzera. Galthatu gabe nour cien ere, sar eraci çutien eta ttoto eraci beren su ondouan; guero eman ceren esne eta gastagna çoumbait, hen eresoursa oro. Bena ordian, Jinco Houna, - houra bera çuçun, Joundane Pethirieqi- jeiqui cen, buria argui arguia, eta eran ceren: "Ziec, praoube ciade eta houn: cien aiçouac aldis aberax dira eta gaichto. Jincouaren justicia içan dadila eguinic!”.

Berhala, lurra ikharratu cen; Jésus eta Joundane Pethiri hanti galdu cien, eta bi çaharrac erori cien belharico... Bihamen goician, nouiz ere nahi içan beitzien jouan heriala jaquitera cer igan cen, etzien ikhousi etcheric batere; hen lekhian, bacen, isla bat, çouin edireiten beita oano egun Barbazeneco heriaren eta Saint-Bertrand-de-Comminges deitzen den hiriaren artian.

Behar die sokhoritu beharetan dienec.

\section{Tantougouen ichtoria çahara}

Tantugou duçu guiçoun çahar bat oihanetan gordez egoiten dena. Béguiatzen tiçu alhorrac eta sohouac amorecatic eta deusere han ebax ahal estezen. Nouis ere oguiac edo belhara ebakiric beitia, agniqueta oro bilduric içan artino naousiaz, Tantugou estuçu lotzen batere; bethi iratzaric diagoçu. Haren ikhousteco loxac eretenitzen tiçu ouhougnac. Houn duçu, ouste ukhen eçaçu, bena duçu basa.

Laborari batec, çougnec beitzakian gaiça ounxachko, eraiten zian usu bere semer Tantugou cela segurki ere lurreco fruten beguiaçaleric hobena.

Ustarilaco aristi batez, ardi saldo bat alhen ari cen mendi batetan; artçagna louac hartu cian: iratzartziarequi, ikhousi cian Tantugou oïhanialat burus ihesi jouaiten. Hala hala eçaguturic içan cen artçagnez, ibar eijer batian, etçanic çagouelaric botche baten gagnian.

Egungo egunian orano, Bortu (Pyrénées) oundoco heri çoumbaitetan, haourac loxa dira harentaco. Nouis ere mothico edo nescatila tchipi batec espeitu nahi obeditu bere amari, hounec eraiten dero: "Abisa hadi! Oihu eguiten diat Tantugouri, eta berhala ginen duc oihaneti."

Aguer, 36 ans, né à Licq-Atherey

Fait sans la collaboration de personne 
Bassen lehen, Pyrénées deitcen dien borthu khantuco herri batetan guiçon bat eta emaste bat, hanitch sahar. Etsien etche tchipi bat, baatse bat eta béhi bat baïcic. Béna çoumbat nahi praoubé issanagatic laguntsen sutien beac béno praoubiagouac. Egun bates lura gordéic sen elhures eta kharou beltsa sen. Herrico jentiac beotsen sien jaten eta édaten aï siélaïc, abeats sien eta ious. Ulhuntsian hurruntic jiten sien bi bidéjant nahi issan sien ukhuatu lekhu hartan, céen gossé eta égari beitsien; jo sien bortha batetan, gueo besté batetan, eta oano bestélibatetan; ihourqué etsutien sartcea utsi nahi ukhen ez deusée jatéa éman nahi ukhen. Orotaric igoriac, chacurac sines gaïstiac ondotik jaraïquiten, bi arotsek etsaquien nouat jouan nouis eta heltu beitsien hiriaren basterila, guiçon éta émaste praoubé hen etche aïtsinila. Hen plagnien entsutiaéqui senhara leihoula jouantsen hen deitseco, eta haen émastia salhé jouan cen borthaen sabaltséa. Her galthatu gabe nour sien, sar éassi sutien eta jar éassi suthondouan, guéo éman cen esné eta gastagna çoumbaït, hec ciéna oö. Béna ordian Goure-Jaouna, -houa cen Joundané Pétiriéqui-, jeiqui cen, buia argui arguia éta éran cen: "Ciec praoubé eta houn sidé; cien aissouac abéats eta gaïsto dia. Jinco hounaen justicia eguinic issan dadiela! Haïn sari lura ikhaatu cen, Jésus eta Joundané Pétiri gordé sien eta bi saharac belhaïcatau sien. Bihamen goissan, hérilat jouan nahi issan sienian, jaquitéco cer heltu sen, etsien icoussi etcheric bate; hen lekhian bacen losko handi bat çouina oano eé édieiten beita Barbazan deitsen den hériaen éta St Bertrand-de-Comminges deitcen den hiiaen artian. Béhar dia laguntu maléousac.

\section{oOo}

Barbaou sahar bat da oihanetan gordatsen dena. Béguiatsen tu alhorac eta sohouac hœtan deuséré ebats estecen. Nouis éta oguiac moustuic beitia edo belhara eta ébaquic buüssaguiac oro sartu artino Barbaou esta lotsen cazu éguiten du bethi. Haen aguertcen icousteco lotsac béguiatsen tu ouhougnac. Houn da, sinhets essassie; bena bassa da. Hanits gaïca saquien laboaï batec éraiten sien ussu béé semer Barbaou sela ségur lurreco frutien béguiassaléric hobena.

Ustailaco arasti bates, ardi saldo bat alha sen bortian: artsaina lotu sen, iatsartziarequi, icoussi sian Barbaou escapatzen oyhanilat buüs. Essagutuic izan sen éé cabalé sagnés, naba eijer batetan, harroca baten gagnen etsanic.

Oano égun Pyrénées deitsen dien bortu khantuco héri çoumbaitetan, haourac haen lotsa dia. Nouĩs eta mothico tchipi batec edo nescatila tchipi batec espeitié ama sinhesten, éraïten déio: Abisa hadi! Deitcen diat (si c'est un garçon), Deitcen dignat (si c'est une fille) Barbaou eta behala oïhanetic jien duc (si c'est un garçon), jinen dun (si c'est une fille).

Nicibar, 32 ans, né à Ossas-Suhare Coll.: Mendiondo, Mathieu 
Bacen beste orduz borthu ondouetaco herri batetan guiçoun bat eta emazte bat çouin hanitz çahar beïtciren. Etcien etche tchipy bat, baratce bat eta behi bat baïcic. Bena çoumbat nahi praübe ciren, sokhorritcen citicien bethi berac beno praübiagouac. Egun batez, lurra gorderic cen elhurrez eta kharrountatcen cian harrien erdiratcialano. Herrico jentiac oro berotcen ciren, jaten eta edaten ari cirelaric; aberats eta irous ciren. Ulhun gnabarrian, bi bidejant hurrunetic horra cirenac ekhuratu nahi içan ciren lekhu hartan, ceren eta hotz eta gosse beitciren; jo cien bortha batetan, guero beste batetan eta beste çoumbaïtetan orano: ihourc ere etçutian utci nahi ukhen sartcera ez eta ere deüsere eman jatera. Ohilturic orotaric, hor oldartiez lastercaturic, bi arrotcec etçakien nourat jouan, nouis eta ere aguitu beitciren herriaren bazterrian, guiçoun eta emazte praübe ciren hen etchiaren aïtcinian. Hen couintac entçunic, senharra aguertu cen leïhouala hen deïtceco, eta haren emaztia jouan cen bertan bortharen çabaltcera. Her galthatu gabe nour ciren, sar eraci eta jar eraci çutien beren su bazterrian; guero cerbutchatu ceyen ezne eta coumbait gaztagna, berec ciena oro. Bena ordian goure Jinco Jaüna, houra cen, Jondone Pethirireki, chutitu cen, buria oro distiraturic, eta erran ceyen: "Ciec, praübe eta houn ciadeyé; cien aïçouac aberats eta gaïchto tuçu. Jincouaren justicia eguin bedi!" Haïmbestenarequi, lurra ikharatu cen, Jesus eta Jondone Phetiri galdu ciren eta bi çaharrac belharico erori... Biharamun goiçan, nahi içan cirenian jouan herriala, jakiteco cer igaran cen, etcien ikhousi etcheric batere: hen lekhian bacen phutçu bat, çouin orano egun ere edireiten beïta Barbezaneco herriaren eta san Bertrand-de-Commingeseco hiriaren artian.

Behar dira laguntu beharrunantac.

\section{oOo}

Tantugou duçu guiçoun çahar oyhanetan gordatcen den bat. Beguiratcen tiçu alhorrac eta sorhouac han deüs ebats eztecen. Nouiz ere ereïtciac ebakiric beitira edo belharra thaillaturic, naüssiaz hourac bilduric içan artino. Tantugou eztiçu louac hartcen; ailhor duçu bethi. Haren aguertcen ikhousteco lotsac etchekitcen tiçu ouhougnac. Houn duçu, sinhets açu, bena bassa duçu. Laborari hanitch gaïca çakian batec erraïten ciçun usu bere semer Tantugou cela segurki lurreco fruten beguirari hobena.

Uztarilaco arrastiry batez ardi saldo bat alhen ari çuçun mendi batetan; artçagna louac hartu ciçun; iratçartcian ikhousi ciçun Tantugou oyhanialat burus ihessi jouaïten. Içan çuçun orobat eçaguturic artçagnez ibar eijer batetan, botchu baten gagnen etçanic.

Egunco egunian, orano bortu ondouetaco herri çoumbaïtetan haürrac lotsa tuçu harentaco. Nouis ere mouthico tchipi batec edo nescatila tchipy batec ezpeitu nahi amac errana eguin, harec erraïten dioçu: Abisa hadi! deittcen diat Tantugou eta berhala jinen duc oïhanetic. 


\section{Barbazaneco iracourgaria edo aleguia}

Bacien lehen ago Pyreneetaco heri batetan guiçoun bat eta emazte bat hanitch çaharrac. Etcien etche tchipi bat, baratce bat eta behi bat baicik. Bena çoumbat nahi praube ciren, urgaizten cutien bethi berac beno beharragouac. Egun batez lurra elhurez tapaturic cen eta kharountatcen cian harrien phorrokatuz. Herrico gentiac oro berotcen cien jatiarequi eta edatiarequi; aberats eta dohain houneco cien. Ulhuncian bi bidajant hurruntic jinic nahi içan ciren baratu gune hartan ceren eta hotz eta gose beitciren; jo cien bortha bat, guero beste bat eta besteric çoumbait; ihourc ere etçutien hartu, ez eta deusere eman jatera. Orotaric ohilturic tchacur errabiatu elibat aztalian, bi arrotzec etçakien nourat jouan, nouiz eta heltu beitciren herri bazterriala guiçoun eta emazte behar hen etchera. Entçutiareki hen plagniac, senharra jarri cen leihouan hen deitceco eta haren emaztia jouan cen fite bortharen çabaltcera. Galthatu gabe nour ciren, sar eraci eta jar erazi çutien sukhaltian: guero eman ceren ezne eta gaztagna çoumbait, çutienac oro. Bena ordain, Goure Jauna, houra cen Joundane Phetirireki, jeiki cen buria oro argui eta eran ceren: "Ciec praube eta houn cirade; cien aiçouac aberats eta gaichto. Jincouaren justicia dela eguinic." Ber mementouan lura ikharatu cen. Jesus eta Joundane Phetiri galdu ciren eta bi çaharrac belharico jarri. Biheramen goician jouan nahi içan cirenian herriala jakitera cer igaran cen etcien ikhoussi etcheric batere: hen lekhian bacen losko handi bat egun orano agueri dena Barbazaneco herriaren eta Saint Bertrand Commingeco hiriaren artian.

Behar dira dohacabiac sokhorritu.

oOo

Tartaroua giçon çahar bat, oyhanetan gordatcen dena. Beguiratcen tu alhorrac eta sohouac deusere ebats eztadin. Oguiac ebakiric edo belhara mouzturic denian, oro bil artekan nausiac, Tartaroua ezta lotcen, beleatz diago bethi. Haren aguertceco lotsac baratcen tu ouhougnac. Houn da sinhets eçacie, bena bassa da. Laborari batec gaiça hanitch çakianac erraiten cian ardura bere semer Tartaroua cela segurki lurreco hountarçunen beguiraçaleric hobena.

Uztarilaco arrastiri batez, ardi saldo bat alhatcen cen bortu batetan; artçagna lothu cen: iratçartcian, ikhoussi cian Tartaroua ihessi jouaiten oihanialat. Içan cen ber guisan eçaguturic artçagnez arbailla eïger batetan etçanic botchu batetan.

Orano egun, goure bortietaco herri çumbaitetan haurrac haren loxa dira. Nouiz eta mothico edo nechcatila ttipi batec ezpeitu nahi mania bere amari erraiten dero: "Abisadi! deitcen diat Tartaroua eta behala giten duk oïhanetic. 
Baçuçun lehenago Pyrénéetaco herri bat'étan guiçoun bat éta émasté bat hanitch çahar béitcien. Etcicien etché tchipi bat, baatce bat éta béhi bat baïcik. Bena, çoumbat nahi praoubé cien, sokhorritcen citicien béthi béak béno praübiagouac. Egun batez, lurra élhourrez gordéik cen éta kharountatxen ciçun harrien erdiatcera. Herriko gentiak oro béotcen cien, jates éta édates, abeats éta ious çututçun. Ulhuncé beyan, bi bidajant, hurruntic giten cienac, nahi içan çututçun ukhuatu lekhu hartan, céen hots éta gose béitcien; jo cicien bortha bat, guéo besté bat, éta bestéik orano: ihourkere etciticien nahukhen utci sartcera, ez éta déüzere eman jatera. Igorriik ootaik, hotçamanik hor gaïstutik cienez, bi arrotcek etçakizien nouat jouan, nouis éta édien beitciaan herrian akabatcian. - Entçutés hen plagnik, senhara leyhoula aguertu çuçun hen déitceco, éta haen emastia bertan jouan çuçun borthaen çabaltera. Galthatu gabé nour cien, saréaci citicien éta ttottoaci suthondouan; gueo cerbuchatu cieçun ezne éta gastagna coumbaï, çutienak oro. Bena - ordian, goué Jinco jaouna - houa çuçun Joundane Phetiekijéiki çuçun buia argui arguia, éta erran cieçun: "Ciec, praubé éta houn cidé; cien aïcouak abéats éta gaichto tutçu. Jincouaren justicia éguin dadila." Haïn çarri, lurra lurra ikhaatu çuçun, Jésus éta Joundané Péthi galdu çututçun éta bi çaharrac belhaico éoi çututçun. Bihamen goïçan, nouis éta jouan nahiçan béitcien herriala jakiteco cer igan cen, etcien ikhousi etchéik baté, béré lekhian baçuçu loskobat sungn édienten béita oano egun Barbazanéco éta Sen Bertran de Comminges-co herrien artian.

Jente écin heltier heltua éguin béharciéçu.

\section{oOo}

Tantugou guiçoun çahar bat duçu gordatcen béita oyhanetan. Beguiatcen tiçu alhorrac eta sohouac déüze ebats estecen. Nouis éta oguiac phikaturic béitia éta belharra ébakiik, buruçaguiac oro bil artio, Tantugou estuçu bate lotcen, bethi kazu eguiten diçu. Haen ikhousteco lotxac ouhougnac béguiatcen tiçu. Houn duçu, sinhetz ecacie, bena salbage duçu. Laboaï batec, gaiça hanitch sakinac, erraïten ciçun uçu béé sémer Tantugou céla segurki lurreco fruten béguiaçaléik hobéna.

Ustaïlaen arristi bates, ardi saldo bat alha aï çutuçun mendi baten gagnen; artçagna lothu çuçun; iatçartcian apercebitu ciçun Tantugou oïhanilat bus eskapatcen. Içan çuçun bardin éçagutuik cecenkaiez, naba éjer bat etan, botchu baten gagnen.

Egun oano, Pyrénéetaco herri çoumbaï étan haourrak haen lotxatuçu. Nouis éta mothiko batec édo neskatila tchipi batec sinhetzi nahi estianian be ama, erraïten dioçu: "Abizadi hii! déitcen dut Tantugou, eta, memento béian, jinen da oïhanetic!"

Palu (Jean-Julien), 31 ans, né à Tardets Coll.: Arhex-Agie (Arnaud), Lagune (Germain), Abadie-Escande (Catherine) 


\section{Barbazaneco erran çahara}

Bassen lehen ago Pyrénées bortietaco paropiabatétan, guiçon eta emazte hanits çahar cien elibat. Etsien etche tipi bat baïssic, baatsé bat éta béhi bat. Bena soumbat nahi praube issanic sokhoritcen sutien bethi berac beno prabiagouac. Egun batez lurra eztaliric sen elhurez eta harriac kharountatuic cien. Paropia hartaco jentiac oro berotsen aï cien, jaten éta edaten cielaï; oro aberatz sien eta irouz. Ulhuntcian bi bidajant huruntic jiten sienac nahi issan cien ukhuratu han séren éta hots beitsiren eta gossé. Bortha bat trucatu sien, bériz bestébat eta beztéic eré orano: ihourc éré etsutian nahi ukhen utsi sartcera ez eta éman déüz jatera. Ohilturic orotaric, kharicaco hoez jarraïkiric bi arotsec etsakien nouat jouan, nouiz éta ére heltu beitsien paropiaren bazteriala guiçon hain eta emazte hain etchiaren aitsiniala, sougn praoube beitsien. Haien phenac ikhoussiric eta haien plagniac entsunic, senhara leïhouala aguertu sen her oïhu eguiteco eta emaztia fite jouan sen borthaen sabaltcera. Galthatu gabe taric nour sien sar éassi sutien eta jar éassi sutien béré suthondouan. Guero serbutchatu seren ezne éta gaztagna sutien gaïssetaïc. Béna ordian, goure Jesus-Christ Jaouna - houa sen Joundane Phetirieki, - jeiki sen, buria béré brillantsia oro ekin éta éran seren: "Siec praoubé siadé, béna houn, sien aïssouac abérats dia eta gaïchto. Jincouaren justissia issan dadila!" Ber mementouan, lurra ikhaatu sen, Jésus eta Joundane Phetiri galdu sien, éta bi çaharrac belhaïcatu sien. Bihamenen goïssian nouiz éta éré jouan nahi issan beitsiren heriala jakitera ser igan sen, etsien ikhoussi etche bat éré; hen plassan bassen hour ukhuatu bat, çougn ediréiten beita oano Barbazaneco paropiaen eta Saint-Bertrand-de-Commingeco hiriaren artian. Behar dissu bethi eguin kaïtatia praoubier.

\section{Tantugouen éran cahara}

Tantugou çahar bat da sougn gordatsen béita oïhanetan. Beguiatsen tu alhorrac éta sorhouac ébats ez tessen déuz éré. Oguiac ébakirik dienian edo belharac burussaguiac éaman éartekan, Tantugou esta lotsen: gouaïta bethi. Tantugou ikhous beldurez ouhougna béguiatsen da ébastetic. Houn dussu sinhets essassu, béna bassa dussu. Laboaïbatec hanic gaïssa sakienic éraïten sian ardura béré sémer Tantugou séla segurki lurreco usten éta urien béguiassaléic hobena.

Uztaïlaco aristiibates ardi saldo bat alha sen bortu batetan artsagna louc hartu cian, iatsartsian ikhoussi sian Tantugou oïhanialat jouaiten. Ber guizan essaguturic issan sen ulhagnez, ibar eijer batetan, etsanic har botchu batetan gagnen.

Egun oano, soumbaït paropia Pyrénéetan, haourac haen lotsa dia. Mothico batec edo uruchouna batec amaï maniac eguin nahi estutienian, éraiten déré: "Gogouman, Tantugouy oyheguiten diognat, behala jinen dun oyhanetic

Garby, 29 ans, né à Alos-Sibas-Abense Coll.: Detchandy, Goyhex 


\section{Soule $^{4}$ (région basque) \\ Pays de Soule (Basses-Pyrénées)}

Lénénago bacen Pyrénéco herri batian guiçon bat eta emazte bat hanitz saharrac. Etcicien hontarçunic etché chipibat, baratcebat éta béhibat baicic. Bena, çombat nahi pobré cien, sokhoritcen çutien bérac béno pobréagoac. Egun batez, lura elhurez gordia cen, éta harrien erdiratceco kharroubat éguiten cien. Herrico jentiac oro bérotcen cien jatiarequi éta édatiarequi; abérax cien éta irous. Ulhuntzian, bi bidejant huruntic jiten cirenac nahi içan cien arrestatu lekhu hartan, céren gossiac eta hotsac harturic beytcien, jo cien bortha bat, guero bestebat eta orano beste hanitz: ihourc etsutian nahi içan utsi sartcera, gutiago deuséré eman jatéra. Orotzas cassatiac, chakhur gaiztoez perseguitiac, bi estranjérec etzakiten norat joan, noiz eré ediren beytziren herri basterian guiçon eta emazté pobré hayein etché aytcinian. Entçutiari hen pleintac, senhara aguertu cen leyhora her oyhu éguitéco, éta haren emaztia aldis joan fité bortharen sabaltcera. Batéré galthatu gabé nor ciren, saréraci çutien éta jaréraci béren suthondouan; guéro cerbutchatu ceyen esné eta gastagna çombait, sutienac oro. Béna ordian, gouré Jincoa, - céren houra cen Phétiriequi, - jeyqui sen buria arguis inguraturic, eta éran ceyen: "Ciec pobré ciadé béna houn; cien ayssoac abérax dira eta gaizto. Jinco hounaren justicia éguin dadila!” Berhala lura ikharatu cen, Jésus eta Phétiri galdu cien éta bi saharrac érori belharico... Bihamen goycian, nahi içan cien joan herriala, cer igaran cen jaquitéra, étcicien ikhoussi étcheric batéré: hen lekhian bacen lac bat (silobat hourés gaintica) çoin édireyten beyta orano eygun Barbazaneco herriaren eta Saint-Bertrand-de-Commingeco hiriaren artian. Behar dicie sokhoritu malérousec.

\section{oOo}

Tantugou da genté saharbat oihanétan gordatcen déna. Béguiratcentu alhorac éta sohouac estécen deuséré ébax. Oguiac mousturic dienian éta belharra daillaturic, buruçaguiac oro barnerat bil artino, Tantugouc estu loic éguiten batéré; beillatcen du bethi. Haren aguertcen ikhousteco loxac béguiratcentu ohoiñac. Houn da, sinhex éçacién bena salbaje da. Hanitz gaiça sakien laboraribatec éraiten cien usu béré sémer ségur Tantugou sela lurreco frutien béguiaçaleric hobéna.

Ustarilaco aristiribatez, ardi tropabat alha cen mendibatian; artçaina loac hartu cien: iratçartcian, apercebitu cien Tantugou escapatcen oyhanéco aldérat. Berguisan eçagutia içan cen artçainez mendien arteco lekhu ejer batian, arrocabaten gainen etçanic.

4. Dokumentu hau Sacazeren esku-idazkien artean altxatu dugu: Euskal Herriko kantonamendu batean ez zuen sartu Sacazek, jadanik herri guziek beren bertsioa bazutelakotz. Beraz gaineratiko dokumentu gisa tratatu zuen eta aparte eman zuen ( MS 1144; 115-116 orr.). Zuberoan bildua izan dela dio, besterik gabe. Dokumentua zein herritakoa den ez dakigu. 
Orano egun, Pyrénéco herri çombaitétan, haurac loxa dira harentaco. Noiz mothico chipibatec edo uruchouna chipibatec espeitu nahi obéditu béré amari, éraiten déo: Cassu éguincéçac hiri! Tantugoui oihu éguiten diat éta, istantian, oihanetic jinen duc.

Le brigadier des Forêts Patalagoyty 
Bazututzun lehenago Pyrénées bortitaco herri batetan gizoun bat eta emazte bat izigarri zaharric. Etzizien etche ttipibat, baratze bat eta behi bat baizic. Bena, zoumbat nahi praube ziren, sokhorritzen zitizien bethi hourac beno praubigo zirenac. Egun batez lurra gorderic zuzun elhurrez eta kharrountatzen ari zuzun aski harrien erdirazteco. Herrico jentic oro berotzen ari zututzun jatez eta edatez; aberats zutuzun eta irous. Ulhountzin bi bidajant hurruntic houna cirenac nahi izan zutuzun ukhuratu lekhu hartan, hotz eta gose beitziren; jo zizien bortha bat, gero bestebat, eta besteric orano: ihourc ere etzitizun nahi izan utzi sartzera ez eta ere deusere eman jatera. Orotaric acazaturic, hor gaitz elibatez lastercaturic, bi estranjerec etzakizien nourat joun, nouiz ere ediren beitziren kharrika bazterrin, gizoun eta emazte bat hain praube ziren harien etche aitzinin. Harien plañien entzutez, senharra ezari zuzun leihoun harien deitzeco eta emaztia joun zuzun bertan bortharen zabaltzera. Galthatu gabe nour ziren, sar erazi zitizien eta jar erazi suthondoun; gero, zerbutchatu zirezun ezne eta gaztaña zoumbait, zutienac oro. Bena ordin, Jinco houna - houra zuzun, Joundane Phetirieki, - jeiki zuzun, buria argi argia, eta erran ziezun: "Ziec, praube zidie eta houn, ziren aizouac aberats tuzu eta gachto. Jincouaren justizia egin dadila!" Haimbestereki, lurra ikharatu zuzun, Jesus eta Joundane Phetiri galdu zutuzun eta bi zaharrac erori zutuzun belharico. Biharamen goizan, nahi zirenin kharrikala joun, jakitera zer igaran zen, etzizien ikhousi etcheric batere: harien plazan bazuzun losco handibat, zouin edireiten beita egun orano Barbazan-eco herriren eta Saint-Bertrand-de-Comminges-eco hirien artin.

Malerousec lagundu behar die.

\section{oOo}

Tantugou gizoun zahar bat duzu oihanetan gordatzen dena. Begiratzen tizu alhorrac eta sohouc deusere ebats eztezen. Ogic mouzturic direnin edo belharra ebakiric, buruzagic oro sar artino, Tantugou eztuzu lotzen; bethi iratzarriric duzu. Haren agertzen ikhousteco lotsac baratzen tizu ouhouñac. Pherestu duzu, sinhets ezazie, bena basa duzu. Gaiza hanitch zakin laborari batec erraiten ziezun usu bere semer Tantugou zela segur lurreco fruten begirazaleic hobena.

Uztarilaco arrastiri batez, ardi saldo bat alha arizutuzun borthu batetan; artzaña louac hartu zizun; iratzartzieki ikhousi zizun Tantugo ezcapatzen oihanilat buruz. Ber gisala ezagutu zizien artzain elibatec, ibar ejer batetan, etzanic botchu batetan gañen.

Egun orano, Pyrénées bortietaco herri zoumbaitetan, haurrac haren lotsa tuzu. Mothico edo nescatcha ttipi batec eztinin nahi bere ama sinhetsi, erraiten diozu: Abiseic hi! Deitzen diat Tantugou eta mementin, jinen duc oihanetic! 
